\title{
SEARCH FOR EVIDENCE OF SUPERSYMMETRY IN THE LIKE-SIGN DIMUON CHANNEL AT THE D $\varnothing$ EXPERIMENT
}

\author{
By
}

Adam Yurkewicz

\section{A DISSERTATION}

Submitted to

Michigan State University

in partial fulfillment of the requirements

for the degree of

DOCTOR OF PHILOSOPHY

Department of Physics and Astronomy

2004 


\section{ABSTRACT \\ SEARCH FOR EVIDENCE OF SUPERSYMMETRY IN THE LIKE-SIGN \\ DIMUON CHANNEL AT THE D $\varnothing$ EXPERIMENT}

By

Adam Yurkewicz

Supersymmetry (SUSY) is a proposed symmetry between fermions and bosons. If this symmetry does exist, it is clearly broken since only half of the particle spectrum is observed. One model which provides a simple breaking mechanism is called minimal supergravity (mSUGRA) inspired SUSY. One clean final state predicted by this model is a trilepton final state from chargino and neutralino decays.

In this analysis, these events are sought in $239 \pm 16 \mathrm{pb}^{-1}$ of DØ Run II data by requiring like-sign dimuon pairs. Requiring only two muons increases the signal acceptance, and adding the like-sign requirement reduces the Standard Model background from Drell-Yan dimuon pairs and the various resonances in the dimuon spectrum. The reach into some parts of mSUGRA parameter space will be greater when searching with the like-sign dilepton final state than the trilepton final state.

Combining results from a search in the like-sign dilepton channels with searches in the trilepton channels provides increased sensitivity in the search for supersymmetry. In this dissertation, the best-ever $\mathrm{D} \varnothing$ limit on the total cross section for associated chargino and neutralino production with leptonic final states is presented. 
for Katie 


\section{ACKNOWLEDGMENTS}

First, I'd like to thank my thesis advisor, Jim Linnemann, who helped me from my recruitment visit to MSU to the final days of writing this dissertation. During our time working together on the L2 trigger and this analysis, he taught me an immense amount, while convincing me that I still have a lot to learn.

I'd like to thank professors Brock, Weerts, Abolins, and Pope for being the rest of the core of the excellent MSU D $\varnothing$ group, and allowing me to be a part of it. Thanks to Dan Edmunds for helping me with my paddle board project. I'd also like to thank the other members of my thesis committee: professors Repko, Mahanti, and Sherrill.

This analysis was a collaboration with Reiner, Roger, and Jim, with early help from Serban. I owe them gratitude for their technical help with the analysis as well as their advice and wisdom. Many others in the New Phenomena group helped me as well, including Volker, Gustaaf, Greg, Ulla, Ryan, Marc, and Meta.

I was lucky to work alongside Reiner, Reinhard, Roger, Dugan, Bob, Dylan, Josh K., Joe, and Josh D. from MSU, and Christos, Marc, Miroslav, Jodi, and James from L2. It was great working with all of you!

Thanks to everyone else in the $\mathrm{D} \emptyset$ collaboration that I didn't mention here by name. This dissertation wouldn't be possible without your years of hard work.

While at MSU, I studied (and didn't study) with Josh, Joy, Dan, Cluze, and C-A. Thanks for your friendship, knowledge, jokes, company, and weekly dinners.

Thanks, Mom and Dad, for a lifetime of unconditional love. Knowing I can always count on it has helped me immeasurably in my life.

Thanks to Charlie for being a great brother, my lifelong friends Dave and Mansoor for being my lifelong friends, and all three for the Turkey Bowl.

And finally, thanks to Katie for her love, support, and encouragement over the past five years, and for being my friend and companion in life. 


\section{Contents}

1 The Standard Model and Supersymmetry 1

1.1 The Standard Model . . . . . . . . . . . . . . . . . . . 2

1.1.1 Gauge Symmetries ... . . . . . . . . . . . 5

1.2 Limitations of the Standard Model . . . . . . . . . . . . . . . . . . 7

1.3 Supersymmetry as an Extension to the Standard Model . . . . . . . . 9

1.4 Sparticle Spectrum . . . . . . . . . . . . . . . . . . . 10

$1.5 \mathrm{mSUGRA} \ldots \ldots \ldots \ldots \ldots \ldots$

1.6 The Trilepton Final State . . . . . . . . . . . . . . . . . . . . . . . . . . . . . . . 13

1.7 Like-Sign Dimuon Channel . . . . . . . . . . . . . . . . . . . . 14

2 The Fermilab Accelerators and the DØ Detector 15

2.1 The Fermilab Accelerators . . . . . . . . . . . . . . . . . . 15

2.1.1 The Preaccelerator . . . . . . . . . . . . . . . . 16

2.1 .2 The Linac . . . . . . . . . . . . . . . . . . . . . . . . . 17

2.1.3 The Booster . . . . . . . . . . . . . . . . . . . 17

2.1 .4 The Main Injector . . . . . . . . . . . . . . . . 18

2.1.5 The Antiproton Source . . . . . . . . . . . . . . . 18

2.1.6 The Tevatron . . . . . . . . . . . . . . . . . . . . 19

2.1.7 The Recycler . . . . . . . . . . . . . . . . . . . . . . . 19

2.2 The DØ Detector . . . . . . . . . . . . . . . . . . . . 19

2.2.1 Coordinate System . . . . . . . . . . . . . . 21

2.2.2 Luminosity . . . . . . . . . . . . . . . . . . . 22

2.2.3 Silicon Microstrip Tracker . . . . . . . . . . . . . . 23

2.2.4 The Scintillating Fiber Tracker . . . . . . . . . . . . . 26

2.2 .5 Preshower Detectors . . . . . . . . . . . . . . . 28

2.2.6 Calorimeter . . . . . . . . . . . . . . . . . . 30

2.2.7 Muon System . . . . . . . . . . . . . . . . . . . . 37

2.3 The DØ Trigger and Data Acquisition Systems . . . . . . . . . . . 41

2.3.1 The Level I Trigger . . . . . . . . . . . . . . . . . . . . . . . . 42

2.3.2 The Level II Trigger . . . . . . . . . . . . . . . . . . . . . . . 45

2.3.3 The Level III Trigger and Data Acquisition . . . . . . . . . . . 48 
3 Offline Event Reconstruction $\quad \mathbf{5 0}$

3.1 Track and Vertex Reconstruction . . . . . . . . . . . . . . 51

3.1 .1 Track Algorithm . . . . . . . . . . . . . . . . 51

3.1 .2 Selecting the Primary Vertex _... . . . . . . . 53

3.1.3 Bottom-Quark Jet Identification . . . . . . . . . . . . . . 53

3.2 Muon Reconstruction . . . . . . . . . . . . . . . . . . . . . . 54

3.2 .1 Muon Types and Qualities . . . . . . . . . . . . . 55

3.2 .2 Muon Isolation . . . . . . . . . . . . . . . . . . . 57

3.3 Jet Reconstruction . . . . . . . . . . . . . . . . . . . . . . . 58

3.3 .1 Jet Cone Algorithm . . . . . . . . . . . . . . . . 58

3.3 .2 Jet Energy Scale . . . . . . . . . . . . . . . . . . . . 59

3.4 Electromagnetic Object Reconstruction . . . . . . . . . . . . 60

3.5 Missing Transverse Energy Reconstruction . . . . . . . . . . . . 61

4 Analysis 63

4.1 Data Set . . . . . . . . . . . . . . . . . . . . . 63

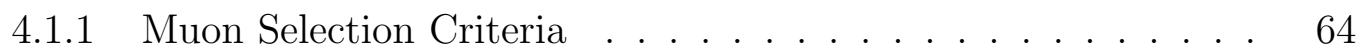

4.2 Trigger Efficiency . . . . . . . . . . . . . . . . . . . 65

4.2.1 L1 Muon Trigger Efficiency _. . . . . . . . . . . . 66

4.2 .2 L2 Muon Trigger Efficiency . . . . . . . . . . . . . . . . . . 69

4.2 .3 L3 Muon Trigger Efficiency . . . . . . . . . . . . . . . . 69

4.2 .4 Total Trigger Efficiency . . . . . . . . . . . . . . . . . . 69

4.3 Reconstruction Efficiencies and Smearing in Data and Monte Carlo . 70

4.3.1 Monte Carlo Momentum Smearing . . . . . . . . . . . . 70

4.3.2 Tracking Efficiency . . . . . . . . . . . . . . . . . 71

4.3 .3 Local Muon Efficiency . . . . . . . . . . . . . . . . . . . . 71

4.3.4 Muon Reconstruction Efficiency . . . . . . . . . . . . . . 71

4.4 Backgrounds . . . . . . . . . . . . . . . . . . . . . . . . . . 73

4.4.1 Background From $\mathrm{b} \overline{\mathrm{b}} / \mathrm{c} \overline{\mathrm{c}}$ Production . . . . . . . . . 76

4.4.2 Sign Misidentification Background . . . . . . . . . . . . 83

4.5 Signal Monte Carlo . . . . . . . . . . . . . . . . . . . . . . . . 84

4.6 Analysis Cuts . . . . . . . . . . . . . . . . . . . . . . . 87

4.6.1 Summary of Analysis Cuts . . . . . . . . . . . . . . 92

4.7 Comparison of Expected Background to Data . . . . . . . . . . . 93

4.8 Combining Results with Other Channels . . . . . . . . . . . . . 98

4.8.1 CLS Method . . . . . . . . . . . . . . . . . . 100

4.9 Cross Section Limit . . . . . . . . . . . . . . . . . . . . . . . . . . 102

5 Conclusions 106

5.1 The Future of the Analysis at D $\varnothing \ldots \ldots \ldots \ldots$

5.2 The Future Beyond D $\varnothing \ldots \ldots \ldots$ 
A L2 Global Worker $\quad 110$

A.1 Input . . . . . . . . . . . . . . . . . . . . . 111

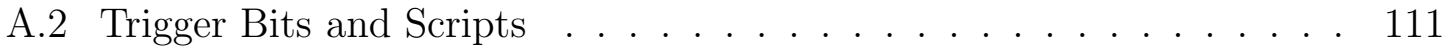

A.3 Tools and Filters . . . . . . . . . . . . . . . . . . . . . . . . 113

A.3.1 Tools and Filters Currently Available . . . . . . . . . . . . 114

A.3.2 Tools and Filters Used in this Analysis . . . . . . . . . . . 114

A.4 Output . . . . . . . . . . . . . . . . . . . . . . . . . 119

A.5 Code Design and Structure . . . . . . . . . . . . . . . . . 119

$\begin{array}{ll}B \text { L2 Error Logger } & 121\end{array}$

B.1 Motivation . . . . . . . . . . . . . . . . . . . . . . . 122

B.2 Architecture . . . . . . . . . . . . . . . . . . . . . . . 123

B.3 User Interface . . . . . . . . . . . . . . . . . . . . . . . . 124

C Paddle Board for Hardware Scalers 126

Bibliography . . . . . . . . . . . . . . . . . . 131 


\section{List of Figures}

1.1 Feynman diagram showing the electromagnetic interaction between two electrons mediated by the exchange of a photon. . . . . . . . . .

1.2 Evolution of the coupling strengths as a function of energy in the Standard Model, where $\alpha_{1}$ corresponds to U(1) (electromagnetic force), $\alpha_{2}$ corresponds to $\mathrm{SU}(2)$ (electroweak force), and $\alpha_{3}$ corresponds to $\mathrm{SU}(3)$

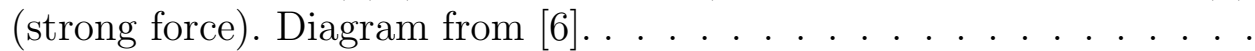

1.3 The evolution of the coupling strengths as a function of energy in supersymmetry, where $\alpha_{1}$ corresponds to U(1) (electromagnetic force), $\alpha_{2}$ corresponds to $\mathrm{SU}(2)$ (electroweak force), and $\alpha_{3}$ corresponds to

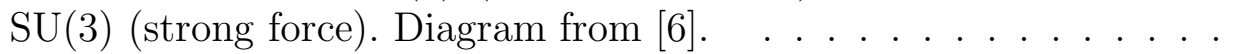

1.4 Feynman diagrams showing chargino $\left(\tilde{\chi}_{1}^{ \pm}\right)$and neutralino $\left(\tilde{\chi}_{2}^{0}\right)$ production and decay to three leptons and other particles including neutralinos $\left(\tilde{\chi}_{1}^{0}\right)$ through a) intermediate smuons $(\tilde{\mu})$ and b) intermediate virtual gauge bosons . . . . . . . . . . . . . . . .

2.1 Schematic diagram of the Fermilab accelerator chain. . . . . . . . 16

2.2 The $\mathrm{D} \varnothing$ detector, including the tracking system, calorimeter, and muon system. . . . . . . . . . . . . . . . . .

2.3 The $\mathrm{D} \varnothing$ tracking system surrounding the beam pipe, including the silicon barrels and disks and the fiber tracking. . . . . . . . . . .

2.4 Diagram of the $\mathrm{D} \varnothing$ silicon barrel detector. . . . . . . . . . . .

2.5 An F disk, part of the $\mathrm{D} \varnothing$ silicon detector. Each of the 12 individual wedges is approximately $7.5 \mathrm{~cm}$ in length. . . . . . . . . .

2.6 Photograph of wedge used in $\mathrm{F}$ disk in the $\mathrm{D} \varnothing$ silicon detector. The wedge is approximately $7.5 \mathrm{~cm}$ in length. Also visible are the SVX II chips used to read out the detector. . . . . . . . . . . . . .

2.7 a) Diagram showing the location of the $\mathrm{D} \varnothing$ central fiber tracker. b) Closeup view showing the axial and stereo layers of scintillating fibers attached to detector barrels. . . . . . . . . . . . . . . . 
2.8 Simulation of the transverse momentum $\left(\mathrm{p}_{\mathrm{T}}\right)$ resolution of the tracking system as a function of pseudorapidity for tracks with $\mathrm{p}_{\mathrm{T}}=1,10$,

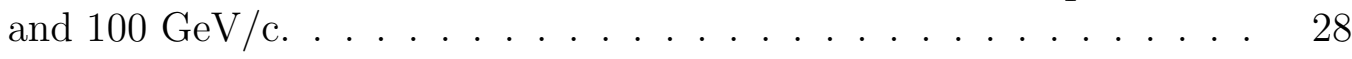

2.9 End view of the $\mathrm{D} \varnothing$ central fiber tracker. . . . . . . . . . . . .

2.10 Insertion of the central fiber tracker into the $\mathrm{D} \varnothing$ detector, inside the calorimeter. . . . . . . . . . . . . . . .

2.11 a) Diagram showing the locations of the central (CPS) and forward (FPS) preshower detectors, outside the central fiber tracker (CFT). b) End view of the CPS, including a closeup view of the three layers of scintillator strips. Not drawn is the tapered lead radiator, which is between the solenoid and the preshower detector. . . . . . . . . .

2.12 The solenoid's radiation and nuclear interaction lengths as functions of pseudorapidity. Diagram from $[22] \ldots \ldots$. . . . . . . . .

2.13 Thickness of lead needed to yield a constant $2 \mathrm{X}_{0}$ (2 radiation lengths) for all particle trajectories. Diagram from $[22] \ldots \ldots$. . . . . .

2.14 A portion of the $\mathrm{D} \varnothing$ central preshower detector, a thin cylindrical detector mainly consisting of scintillating fibers. . . . . . . . .

2.15 The $\mathrm{D} \varnothing$ calorimeter, composed of layers of heavy metals and liquid argon. . . . . . . . . . . . . . . . . .

2.16 The $\mathrm{D} \varnothing$ calorimeter sitting inside the $\mathrm{D} \varnothing$ detector outside of the collision hall during the period between Run I and Run II. . . . . . . . .

2.17 A D $\varnothing$ calorimeter cell with alternating layers of absorber plates, liquid argon, and signal boards. Many cells form the layers of the calorimeter. Absorber plates can vary in size, but the gap between the absorber plate and the signal board is always $2.3 \mathrm{~mm}$. . . . . . . . . .

2.18 The $\mathrm{D} \varnothing$ muon system, including scintillator (scint) and proportional and mini-drift tube chamber layers (PDT and MDT). . . . . . . . 38

2.19 A muon proportional drift tube unit cell consisting of an anode sense signal wire in a gas-filled chamber and two sets of cathode pads. . . .

2.20 A proportional drift tube unit cell viewed from above. . . . . . . . . . 39

2.21 Part of the C layer of the $\mathrm{D} \varnothing$ forward muon system. . . . . . . . . . 40

2.22 A single 4.5 degree central fiber tracker (CFT) sector along with a hypothetical track and hits in all eight CFT layers and the central preshower (CPS) axial layer. . . . . . . . . . . . . . 
3.1 The muon isolation variable in the calorimeter. The energy contained in a cone of radius 0.1 around a muon is subtracted from the energy contained in a cone of radius $0.4 \ldots \ldots \ldots$. . . . . . . . . .

3.2 The electromagnetic (EM) isolation variable (iso) in the calorimeter. The energy measured in the central preshower (CPS) detector is not used in the calculation. Energy measured in the EM, fine hadronic $(\mathrm{FH})$, and coarse hadronic $(\mathrm{CH})$ layers are used in the calculation. Diagram from $[41] . \ldots \ldots \ldots \ldots$. . . . . . . . . .

4.1 a) The $\mathrm{L} 1$ trigger efficiency as a function of $\mathrm{p}_{\mathrm{T}}$ with respect to loose, $n s e g=3$ muons. b) The L1 trigger efficiency as a function of $\eta$ with respect to loose, $n$ seg $=3$ muons with $\mathrm{p}_{\mathrm{T}}$ greater than $5 \mathrm{GeV} / \mathrm{c}$. . .

4.2 a) The L2 trigger efficiency as a function of $\eta$ with respect to loose, $n s e g=3$ muons with $\mathrm{p}_{\mathrm{T}}$ greater than $5 \mathrm{GeV} / \mathrm{c}$. b) The L3 trigger efficiency as a function of $\mathrm{p}_{\mathrm{T}}$ with respect to loose, nseg $=3$ muons. .

4.3 a) Tracking efficiency as a function of $\eta$ with respect to local muons in the $\mathrm{J} / \Psi$ peak measured in data. b) Tracking efficiency of reconstructed muons as a function of $\eta$ with respect to Monte Carlo (MC) muons.

4.4 Tracking efficiency measured in data divided by the Monte Carlo (MC) tracking efficiency as a function of $\eta$ with respect to local muons in the $\mathrm{J} / \Psi$ peak. . . . . . . . . . . . . . . . . .

4.5 a) The loose muon efficiency as a function of $\eta$ with respect to tracks measured in data. The tracks are from muon-track pairs with an invariant mass that lies under the $\mathrm{Z}$ peak. $\mathrm{b}$ ) The loose muon efficiency as a function of $\eta$ with respect to Monte Carlo (MC) muons. . . . . .

4.6 The loose muon efficiency as measured in data divided by the Monte Carlo (MC) local muon efficiency, as a function of $\eta$ with respect to local muons in the $\mathrm{Z}$ peak. . . . . . . . . . . . . . 75

4.7 The total muon reconstruction efficiency as a function of $\eta \ldots \ldots . .75$

4.8 The measured $\Delta \phi$ between muons in like-sign muon pairs in the data for the cases where both muons are isolated and only one is isolated. .

$4.9 \mathrm{R}$, the ratio of the number of events in the sample with two isolated muons to the number of events in the sample with one isolated muon, as a function of the $\mathrm{p}_{\mathrm{T}}$ of the nearly isolated muon. The line is an exponential function fit to the data which was used to weight the background sample in modeling the $\mathrm{b} \overline{\mathrm{b}}$ background. . . . . . . . . . . . 
4.10 a) Circles show the invariant mass distribution of opposite-sign muon pairs. Backgrounds are described by the legend. b) Circles show the invariant mass distribution of opposite-sign muon pairs after the subtraction of Monte Carlo background estimates. The shaded histogram is the expected remaining background from $\mathrm{b} \overline{\mathrm{b}} / \mathrm{c} \overline{\mathrm{c}}$, which is estimated using like-sign data. . . . . . . . . . . . . . . . .

4.11 Circles show the invariant mass distribution of isolated like-sign muon pairs in data. The shaded histogram is the expected remaining background, from $\mathrm{b} \overline{\mathrm{b}} / \mathrm{c} \overline{\mathrm{c}}$, which is estimated using the nearly isolated likesign data as described in the text. . . . . . . . . . . . . . .

4.12 The $\mathrm{p}_{\mathrm{T}}$ spectrum of opposite-sign muon pairs as measured in data. These muons could be used to estimate the pool of candidates for sign mismeasurement. . . . . . . . . . . . . . . . .

4.13 The cross section times branching ratio into three leptons as a function of the $m_{0}$ parameter. . . . . . . . . . . . . . . . .

4.14 The cross section times branching ratio into three leptons as a function of the mass of the chargino. . . . . . . . . . . . . . .

4.15 The $\mathrm{p}_{\mathrm{T}}$ distribution of muons in the $\mathrm{b} \overline{\mathrm{b}}$ background sample and in $\mathrm{a}$ signal Monte Carlo sample (SUSY point 14). . . . . . . . . . . .

4.16 The $\Delta \phi(\mu, \mu)$ distribution in the $\mathrm{b} \overline{\mathrm{b}}$ background sample and in a signal Monte Carlo sample (SUSY point 14). . . . . . . . . . . . . . . .

4.17 The scaled missing transverse energy distribution in the $\mathrm{b} \overline{\mathrm{b}}$ background sample and in a signal Monte Carlo sample (SUSY point 14). . . . . .

4.18 The distance in $\phi(\Delta \phi(\mathrm{MET}$, Jet $))$ between the direction of the missing transverse energy (MET) in the event and the jet furthest away from the direction of the MET in the b $\bar{b}$ background sample and in a signal Monte Carlo sample (SUSY point 14). . . . . . . . . . . . . .

4.19 The distribution of missing transverse energy multiplied by the $\mathrm{p}_{\mathrm{T}}$ of the lower $\mathrm{p}_{\mathrm{T}}$ muon in the $\mathrm{b} \overline{\mathrm{b}}$ background sample and in a signal Monte Carlo sample (SUSY point 14).

4.20 Event display of the event passing all cuts in data, event number 44096517 from run 177010 . In the middle of the display, reconstructed charged-particle tracks can be seen. The green lines represent muons traversing the detector. . . . . . . . . . . . . . . . . 
4.21 Event display of the event passing all cuts in data, event number 44096517 from run 177010. Reconstructed tracks are visible in the center of the display. The red, orange and green bars are hits in the A, B, and $\mathrm{C}$ layers of the muon system. . . . . . . . . . . . . .

4.22 Event display of the event passing all cuts in data, event number 44096517 from run 177010. Reconstructed tracks are visible in the center of the display. The red, orange and green bars on the outer part of the display are hits in the A, B, and C layers of the muon system. The red and blue blocks represent energy in the calorimeter and the yellow block represents missing energy. . . . . . . . . . . . . .

4.23 Limit on the total cross section for associated chargino $\left(\tilde{\chi}_{1}^{ \pm}\right)$and neutralino $\left(\tilde{\chi}_{2}^{0}\right)$ production in the like-sign dimuon channel as a function of the $\tilde{\chi}_{1}^{ \pm}$mass. . . . . . . . . . . . . . . . . . . . .

4.24 Limits on the total cross section for associated chargino $\left(\tilde{\chi}_{1}^{ \pm}\right)$and neutralino $\left(\tilde{\chi}_{2}^{0}\right)$ production with leptonic final states set by $\mathrm{D} \varnothing$ in Run I (top line) and in this analysis (second from top) in comparison with the expected limit (second from bottom) and the signal cross section predicted for in mSUGRA (bottom line) as a function of the $\tilde{\chi}_{1}^{ \pm}$mass. Chargino masses below $103 \mathrm{GeV} / \mathrm{c}^{2}$ are excluded by direct searches at LEP. The D $\varnothing$ Run I limit was based on searches in the eee, ee $\mu$, e $\mu \mu$, and $\mu \mu \mu$ trilepton final states with between $75 \mathrm{pb}^{-1}$ and $95 \mathrm{pb}^{-1}$ of integrated luminosity. . . . . . . . . . . . . . . . . . . . 105

A.1 Data flow from the detectors to the L2 Global worker. . . . . . . . . 112

B.1 Simplified Unified Modeling Language (UML) diagram showing the major classes in the l2errorlogger package and the relationships between them. . . . . . . . . . . . . . . . . . 122

C.1 The paddle board. Picture courtesy of Kathleen Yurkewicz. . . . . . . 127

C.2 Design diagram of the paddle board, showing the connectors and traces connecting them. . . . . . . . . . . . . . . 128

C.3 A pie chart and corresponding legend showing the amount of time spent in each state for the Global worker. . . . . . . . . . . . . . . . . . 130

IMAGES IN THIS DISSERTATION ARE PRESENTED IN COLOR. 


\section{List of Tables}

1.1 The fundamental forces and the particles (gauge bosons) mediating those forces. . . . . . . . . . . . . . .

1.2 The fundamental particles of the Standard Model and some of their properties. $\mathrm{L}_{e}, \mathrm{~L}_{\mu}$, and $\mathrm{L}_{\tau}$ are the electron, muon, and tau lepton numbers. For every particle there is a corresponding antiparticle. . . . . .

1.3 The fundamental particles of the Standard Model grouped into generations. The main difference between the generations is the masses of their particles. . . . . . . . . . . . . . . . .

1.4 The particles of the Standard Model and their corresponding sparticles in supersymmetric models. . . . . . . . . . . . . . . . . . . . .

4.1 The sizes, calculated cross sections, and integrated luminosities of the Monte Carlo (MC) samples. . . . . . . . . . . . . . . .

4.2 The values for the 20 points in mSUGRA parameter space chosen for study, the cross section times branching ratio to trileptons for each point, the masses of the two neutralinos $\left(\tilde{\chi}_{1}^{0}\right.$ and $\left.\tilde{\chi}_{2}^{0}\right)$, chargino $\left(\tilde{\chi}_{1}^{ \pm}\right)$, and slepton $(\tilde{l})$ for each point, the number of events expected $\left(\mathrm{N}_{\exp }\right)$ for $239 \mathrm{pb}^{-1}$ of data and the efficiency (E) for each point. The mSUGRA parameters are defined in Section 1.5. The sign $\mu$ parameter is always positive. Points in bold are used in setting a limit in Section 4.9. . . .

4.3 Number of events after applying each cut to signal Monte Carlo samples for points 1-12. Total errors (sum of statistical and systematic) are in parentheses. . . . . . . . . . . . . . . .

4.4 Number of events after applying each cut to signal Monte Carlo samples for points 13-20. Total errors (sum of statistical and systematic) are in parentheses. . . . . . . . . . . . . . . . .

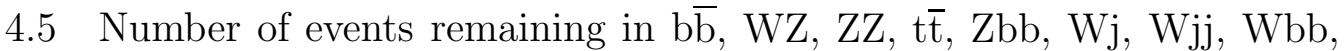
$\mathrm{Z} / \gamma$ background samples after applying each cut to the samples. Total errors (sum of statistical and systematic) are in parentheses. . . . . 
4.6 Number of events remaining in data and the number expected from the sum of all backgrounds after applying each cut. Total errors (sum of statistical and systematic) are in parentheses. . . . . . . . . . .

4.7 Table showing the kinematic values for the two muons and MET in run 177010 , event 44096517 . . . . . . . . . . . . . . . .

4.8 Luminosity, number of observed candidate events, expected number of background events for each final state used in determining the combined cross section limit, and the excluded number of signal events $\left(\mathrm{N}_{\text {excluded }}\right) \ldots \ldots \ldots \ldots \ldots \ldots \ldots$

4.9 For the SUSY points, the cross section times branching ratio to trileptons, the mass of the chargino $\left(\tilde{\chi}_{1}^{ \pm}\right)$, the number of events expected $\left(\mathrm{N}_{\exp }\right)$ and the efficiency (E) for each point for $239 \mathrm{pb}^{-1}$ of data, and the cross section limit in the like-sign dimuon channel $\left(\sigma_{\text {lim }}^{\mu \mu}\right)$ and overall

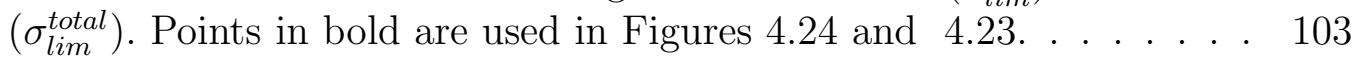

A.1 L2 tools currently available. For each tool, the configurable parameters are listed, along with a brief description [61] . . . . . . . . . . . 115

A.2 L2 tools currently available. For each tool, the configurable parameters are listed, along with a brief description [61] . . . . . . . . . . 116

A.3 L2 filters currently available. For each filter, the configurable parameters are listed, along with a brief description [61]. . . . . . . . . . 117

A.4 L2 filters currently available. For each filter, the configurable parameters are listed, along with a brief description [61]. . . . . . . . . 118

A.5 L2 filters currently available. For each filter, the configurable parameters are listed, along with a brief description [61]. . . . . . . . . . . 119

B.1 The available severity levels in the l2errorlogger. . . . . . . . . . . . . 124 


\section{Chapter 1}

\section{The Standard Model and}

\section{Supersymmetry}

The Standard Model of particle physics is a quantum field theory which encapsulates the knowledge of fundamental particles and forces. It identifies structureless, elementary particles that constitute all the observed matter in the universe - these are the quarks and leptons. The interactions between the quarks and leptons are mediated by particles called gauge bosons (see Table 1.1). The Standard Model provides the theoretical framework to calculate physical (measurable) quantities, explain observed phenomena, and make predictions that can be checked experimentally. Most of these predictions have been confirmed to spectacular precision (many at Fermilab), which has given the Standard Model its name and made it the theoretical framework in which particle physics currently resides.

Despite its many successes, there is consensus among particle physicists that the Standard Model is not the final theory of particle physics. This dissertation is concerned with the search for evidence of supersymmetry, one of the proposed extensions to the Standard Model.

Much of the description of the Standard Model in this chapter draws heavily 


\begin{tabular}{|c|c|c|}
\hline Force & Particles Mediating & Particles Experiencing \\
\hline strong & gluons $(\mathrm{g})$ & quarks, gluons \\
electromagnetic & photon $(\gamma)$ & electrically charged \\
weak & $\mathrm{W}^{+}, \mathrm{W}^{-}, \mathrm{Z}^{0}$ & quarks, leptons \\
gravitation & graviton (unobserved) & all \\
\hline
\end{tabular}

Table 1.1: The fundamental forces and the particles (gauge bosons) mediating those forces.

from references [1-3]. These excellent texts should be consulted for a more thorough introduction to the Standard Model.

\subsection{The Standard Model}

The familiar matter of everyday life is made of molecules. These molecules are built from atoms, which in turn are built from protons, neutrons, and electrons. Protons and neutrons are made of quarks. Electrons and neutrinos are examples of leptons. Quarks and leptons are spin 1/2 fermions. Spin 1 gauge bosons are responsible for the exchange of forces between quarks and leptons. Quarks, leptons, and gauge bosons (and their antiparticles) are the particles of the Standard Model.

The Standard Model is a quantum field theory. It postulates that several fields, one for each type (also called flavor) of particle, permeate all space and interact with each other. Excitations in these fields are observed as particles.

The flavors and properties of the Standard Model particles are summarized in Table 1.2. The quarks and leptons can be arranged in generations (see Table 1.3). The grouping of Table 1.3 highlights the similarities between these generations.

Different combinations of quarks form the spectrum of particles called hadrons. The hadrons are divided into baryons (three quark fermion states) and mesons (two quark boson states). One baryon, the proton, is made from the combination $\{u u d\}$. The charge of the proton (in units of the electron charge) is the sum of the charges 


\begin{tabular}{|c|c|c|c|c|c|c|c|}
\hline Flavor & $\begin{array}{c}\text { Electric } \\
\text { Charge (e) }\end{array}$ & Spin & Mass $(\mathrm{GeV})$ & $\begin{array}{c}\text { Baryon } \\
\text { Number }\end{array}$ & $\mathrm{L}_{e}$ & $\mathrm{~L}_{\mu}$ & $\mathrm{L}_{\tau}$ \\
\hline \multicolumn{8}{|c|}{ Quarks } \\
\hline $\mathrm{u}(\mathrm{up})$ & $+2 / 3$ & $1 / 2$ & $0.0015-0.004$ & $1 / 3$ & 0 & 0 & 0 \\
\hline $\mathrm{d}($ down$)$ & $-1 / 3$ & $1 / 2$ & $0.004-0.008$ & $1 / 3$ & 0 & 0 & 0 \\
\hline c (charm) & $+2 / 3$ & $1 / 2$ & $1.15-1.35$ & $1 / 3$ & 0 & 0 & 0 \\
\hline s (strange) & $-1 / 3$ & $1 / 2$ & $0.08-0.13$ & $1 / 3$ & 0 & 0 & 0 \\
\hline $\mathrm{t}(\mathrm{top})$ & $+2 / 3$ & $1 / 2$ & 178 & $1 / 3$ & 0 & 0 & 0 \\
\hline b (bottom) & $-1 / 3$ & $1 / 2$ & $4.1-4.4$ & $1 / 3$ & 0 & 0 & 0 \\
\hline \multicolumn{8}{|c|}{ Leptons } \\
\hline e (electron) & -1 & $1 / 2$ & $0.511 \times 10^{-3}$ & 0 & 1 & 0 & 0 \\
\hline$\nu_{\mathrm{e}}($ electron neutrino) & 0 & $1 / 2$ & $<3 \times 10^{-9}$ & 0 & 1 & 0 & 0 \\
\hline$\mu$ (muon) & -1 & $1 / 2$ & 0.106 & 0 & 0 & 1 & 0 \\
\hline$\nu_{\mu}($ muon neutrino $)$ & 0 & $1 / 2$ & $<1.9 \times 10^{-4}$ & 0 & 0 & 1 & 0 \\
\hline$\tau(\mathrm{tau})$ & -1 & $1 / 2$ & 1.78 & 0 & 0 & 0 & 1 \\
\hline$\nu_{\tau}($ tau neutrino $)$ & 0 & $1 / 2$ & $<1.8 \times 10^{-2}$ & 0 & 0 & 0 & 1 \\
\hline \multicolumn{8}{|c|}{ Gauge Bosons } \\
\hline $\mathrm{W}^{ \pm}$(charged weak) & \pm 1 & 1 & 80.4 & 0 & 0 & 0 & 0 \\
\hline $\mathrm{Z}^{0}$ (neutral weak) & 0 & 1 & 91.2 & 0 & 0 & 0 & 0 \\
\hline$\gamma($ photon $)$ & 0 & 1 & 0 & 0 & 0 & 0 & 0 \\
\hline $\mathrm{g}_{i}(\mathrm{i}=1, \ldots, 8$ gluons $)$ & 0 & 1 & 0 & 0 & 0 & 0 & 0 \\
\hline g (graviton) & 0 & 2 & 0 & 0 & 0 & 0 & 0 \\
\hline
\end{tabular}

Table 1.2: The fundamental particles of the Standard Model and some of their properties. $\mathrm{L}_{e}, \mathrm{~L}_{\mu}$, and $\mathrm{L}_{\tau}$ are the electron, muon, and tau lepton numbers. For every particle there is a corresponding antiparticle.

\begin{tabular}{|c|c|c|}
\hline Generation 1 & Generation 2 & Generation 3 \\
\hline $\mathrm{u}$ & $\mathrm{c}$ & $\mathrm{t}$ \\
$\mathrm{d}$ & $\mathrm{s}$ & $\mathrm{b}$ \\
$\mathrm{e}$ & $\mu$ & $\tau$ \\
$\nu_{\mathrm{e}}$ & $\nu_{\mu}$ & $\nu_{\tau}$ \\
\hline
\end{tabular}

Table 1.3: The fundamental particles of the Standard Model grouped into generations. The main difference between the generations is the masses of their particles.

of its constituent quarks, $2 / 3+2 / 3+(-1 / 3)=+1$, its baryon number is 1 , and its lepton number is 0 (baryon number and lepton number are additive quantum numbers. See Table 1.2). A neutron is made with the combination $\{u d d\}$. It has electric charge $2 / 3+(-1 / 3)+(-1 / 3)=0$, baryon number 1 , and lepton number 0 . The fundamental 


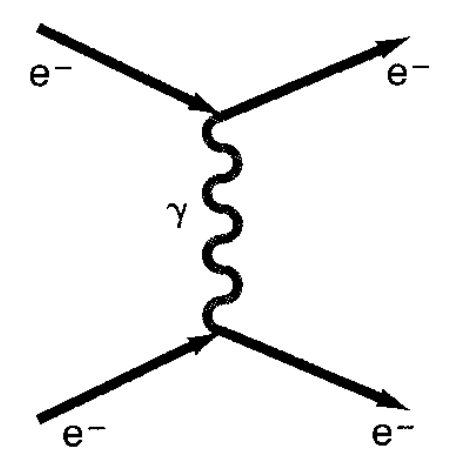

Figure 1.1: Feynman diagram showing the electromagnetic interaction between two electrons mediated by the exchange of a photon.

similarity between neutrons and protons is evident using this scheme. The success of the quark model in explaining and predicting other experimentally observed states led to its acceptance.

There are problems with this simplistic view. The combination $\{u u u\}$ has been observed as the $\Delta^{++}$baryon. The existence of the $\Delta^{++}$presents a problem because its spin, $\mathrm{J}=3 / 2$, is obtained by combining three identical fermions which violates Fermi statistics. Another problem is that neither combinations of more than three quarks or single quarks are observed (Recent results have raised the possibility of the existence of pentaquark states, which were recently predicted to exist [4]).

The solution to these problems is the introduction of another quantum number called color. It can have one of three values, called red (R), green $(\mathrm{G})$, and blue (B), with antiquarks having the values $\overline{\mathrm{R}}, \overline{\mathrm{G}}$, and $\overline{\mathrm{B}}$ (These names have no connection to the colors of the macroscopic world). In this theory, bound states must be colorless, so mesons are formed from one quark and one antiquark which carry a color and its anti-color (like $\mathrm{R} \overline{\mathrm{R}}$ ), while baryons are formed from three quarks, one of each color. Thus the $\Delta^{++}$baryon consists of one u quark of each color.

Interactions between quarks and leptons are mediated by gauge bosons. Photons are the mediators of the electromagnetic interaction. Feynman diagrams like the one in 
Figure 1.1 show pictorially how electromagnetic interactions between particles occur through the exchange of photons.

Color is exchanged between quarks and gluons (both of which carry a color charge), much as electric charge is exchanged between electrically charged particles. Gluons are colored objects that carry color charge as the mediators of the strong force. Unlike photons, which mediate a force that gets weaker with distance, gluons are responsible for a binding force that strengthens with distance. Gluons (and quarks) are colored, so cannot be observed directly because bound states are formed only as colorless objects. This behaviour is called color confinement. Eventually, when colored objects are pulled apart from other colored objects (as occurs in high-energy collisions), the energy released is converted into new colored objects, and colorless objects are formed from groups of the colored objects. Only colorless objects are observed in particle detectors, and they are detected as streams, or jets, of particles. It should be emphasized that leptons do not carry a color charge, and thus do not feel the strong force. This is the main distinction between quarks and leptons.

The weak bosons $\left(\mathrm{W}^{ \pm}\right.$and $\left.\mathrm{Z}^{0}\right)$ mediate the weak force, which is responsible for radioactive decay. The fact that weak gauge bosons are massive, unlike photons, limits the distance over which they are effective. It is currently unknown how the weak gauge bosons acquire masses. One leading theory is the Higgs mechanism (see Section 1.1.1).

\subsubsection{Gauge Symmetries}

The strong, weak, and electromagnetic forces can be described as a combination of three unitary gauge groups, denoted as $\mathrm{SU}(3) \otimes \mathrm{SU}(2) \otimes \mathrm{U}(1)[2]$. The group $\mathrm{SU}(3)$ is the symmetry group of strong interactions, $\mathrm{U}(1)$ describes electromagnetic interactions, and $\mathrm{SU}(2) \otimes \mathrm{U}(1)$ represents the unified weak and electromagnetic interactions.

The symmetries referred to are gauge (phase) symmetries. These symmetries are of prime importance in particle physics. To illustrate, consider that observables depend 
on the wave function squared $\left(|\Psi|^{2}\right)$ and not on the wave function. Therefore, the transformation

$$
\Psi \rightarrow \Psi^{\prime}=e^{-i \chi(\vec{x}, t)} \Psi
$$

where $\chi(\vec{x}, t)$ is an arbitrary phase which can depend on space and time coordinates, should leave the observables unchanged. That is, the theory should be locally gauge invariant. Physically, since the absolute phase cannot be measured, the choice of phase should not matter. If one successively inserts $\Psi$ and $\Psi^{\prime}$ into the simple Schrödinger equation for a matter particle,

$$
\frac{1}{2 m} \nabla^{2} \Psi(\vec{x}, t)=i \frac{\partial \Psi(\vec{x}, t)}{\partial t}
$$

the equation is clearly not invariant under the transformation of Equation 1.1. If $\Psi$ satisfies the equation, $\Psi^{\prime}$ may not since the derivatives may not cancel.

One can modify the Schrödinger equation to guarantee gauge invariance. For electrically charged particles, the modified Schrödinger equation is

$$
\frac{1}{2 m}(-i \nabla+e \vec{A})^{2} \Psi=\left(i \frac{\partial}{\partial t}+e V\right) \Psi
$$

where $e$ is the electric charge, $V$ is the electric potential, and $\vec{A}$ is the vector potential. With this change, the Schrödinger equation is invariant under the simultaneous transformations (1.1) and

$$
\begin{aligned}
& A \rightarrow A^{\prime}=A+\frac{1}{e} \nabla \chi \\
& V \rightarrow V^{\prime}=V-\frac{1}{e} \frac{\partial \chi}{\partial t} .
\end{aligned}
$$

Thus, the requirement that the theory be locally gauge invariant leads to the required presence of a field $A^{\mu}=(V ; \vec{A})$. Further, since particles are viewed as excitations in fields in this theory, the requirement of gauge invariance also leads to the presence of 
gauge bosons.

In the Standard Model, phase symmetries (mathematically, requirements that the theory be invariant under gauge transformations) are used to guide the construction of theories. The gauge fields and particles follow naturally from these symmetries.

The gauge theories introduced above can involve only massless gauge bosons. However, massive gauge bosons $\left(\mathrm{W}^{ \pm}, \mathrm{Z}^{0}\right)$ have been observed experimentally. Evidence of a mechanism that causes the gauge bosons to acquire their masses is highly sought in particle physics. Currently, the leading candidate is the Higgs mechanism.

Peter Higgs conjectured in 1964 that the massless gauge bosons of weak interactions acquire their mass through interaction with a scalar field (the Higgs Field), resulting in a single massless gauge boson (the photon) and three massive gauge bosons $\left(\mathrm{W}^{ \pm}\right.$and $\left.\mathrm{Z}^{0}\right)[5]$. The assumption is that the universe is filled with a spin-zero field, called the Higgs field. The gauge bosons' and fermions' interactions with this field give them their masses. This interaction, in which the particles gain masses, is an example of spontaneous symmetry breaking, in which the $\mathrm{SU}(2)$ and $\mathrm{U}(1)$ symmetries are broken.

\subsection{Limitations of the Standard Model}

There are indications that the Standard Model is not the final theory of particle physics and that more fundamental physics is left to be discovered. Experiments in the years ahead will give insight into which, if any, of the proposed theoretical extensions to the Standard Model is correct.

One reason for the need to extend the Standard Model is that the gauge bosons are massive. Several theories, including supersymmetry, have been proposed which include the Higgs mechanism to give the gauge bosons masses.

A second reason arises from the fact that several theoretical unifications of forces 


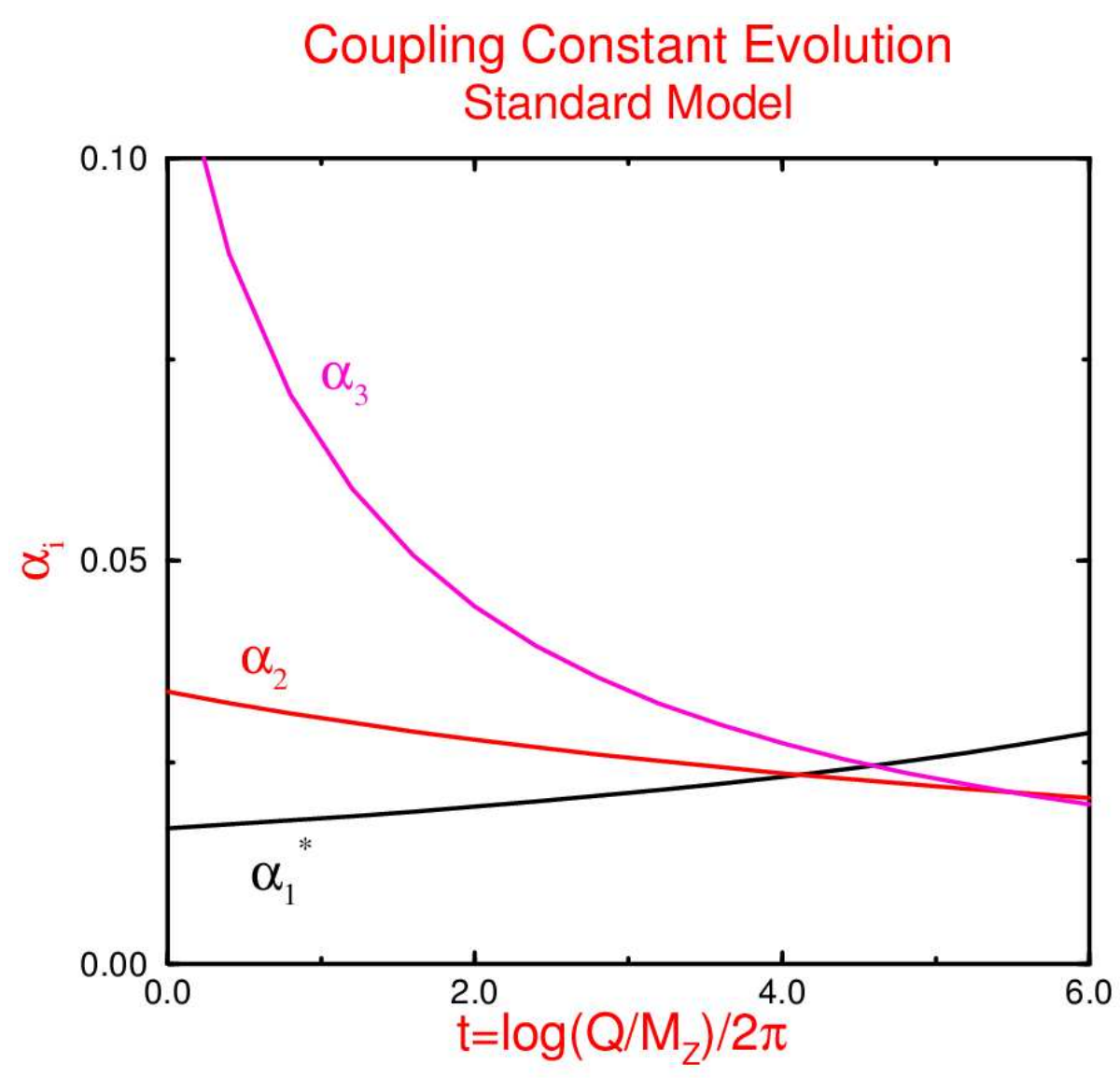

Figure 1.2: Evolution of the coupling strengths as a function of energy in the Standard Model, where $\alpha_{1}$ corresponds to U(1) (electromagnetic force), $\alpha_{2}$ corresponds to $\mathrm{SU}(2)$ (electroweak force), and $\alpha_{3}$ corresponds to $\mathrm{SU}(3)$ (strong force). Diagram from [6].

have already occurred. Electricity and magnetism were once thought of as unrelated, as were the electromagnetic and weak forces. Thus, many theorists expect that a theory that unifies all of the forces should be the final theory. The Standard Model separates the unified electroweak forces from the strong force, and does not include gravity.

The fundamental forces are each characterized by a coupling strength. The coupling strength appears in all physical calculations, and is indicative of the strength of the interaction. These coupling strengths have a dependence on the interaction energy; more precisely, they depend on the momentum transfer between two particles involved in an interaction. Figure 1.2 shows the values of the coupling strengths as 
a function of a parameter that depends on the interaction energy. Theoretical calculations in the Standard Model predict that the coupling strengths are closer at high energies than at lower energies. The experimental data taken so far agrees. However, the coupling strengths will not meet exactly without the existence of some new physics which affects their dependence on the energy. Supersymmetry is one candidate for this new physics.

A third reason for introducing an extension to the Standard Model is revealed when calculations using the Standard Model framework include a Higgs boson. The inclusion of the Higgs boson leads to unphysical results in some calculations. Perturbative calculations of the mass of the Higgs boson squared have quadratic divergences. Infinite terms appear in the sum. It is theoretically possible to introduce counterterms in the perturbation series which cancel these divergences, but this has no physical justification, and has been considered unnatural.

\subsection{Supersymmetry as an Extension to the Stan- dard Model}

Supersymmetry is a proposed symmetry which relates fermions to bosons $[3,6-9]$. It postulates that for every particle there is a corresponding sparticle, identical to the particle but with a spin different by $1 / 2$. If it exists, supersymmetry must be a broken symmetry because no sparticles have been detected with masses equal to the Standard Model particles.

Supersymmetry solves the problems raised in Section 1.2. It includes Higgs bosons to give particles mass. It also modifies the evolution of the coupling constants so that they unify at a high energy (see Figure 1.3). It introduces new terms into the calculation of the Higgs mass squared which cancel the terms that lead to the quadratic divergence. 


\section{Coupling Constant Evolution SUSY Model}

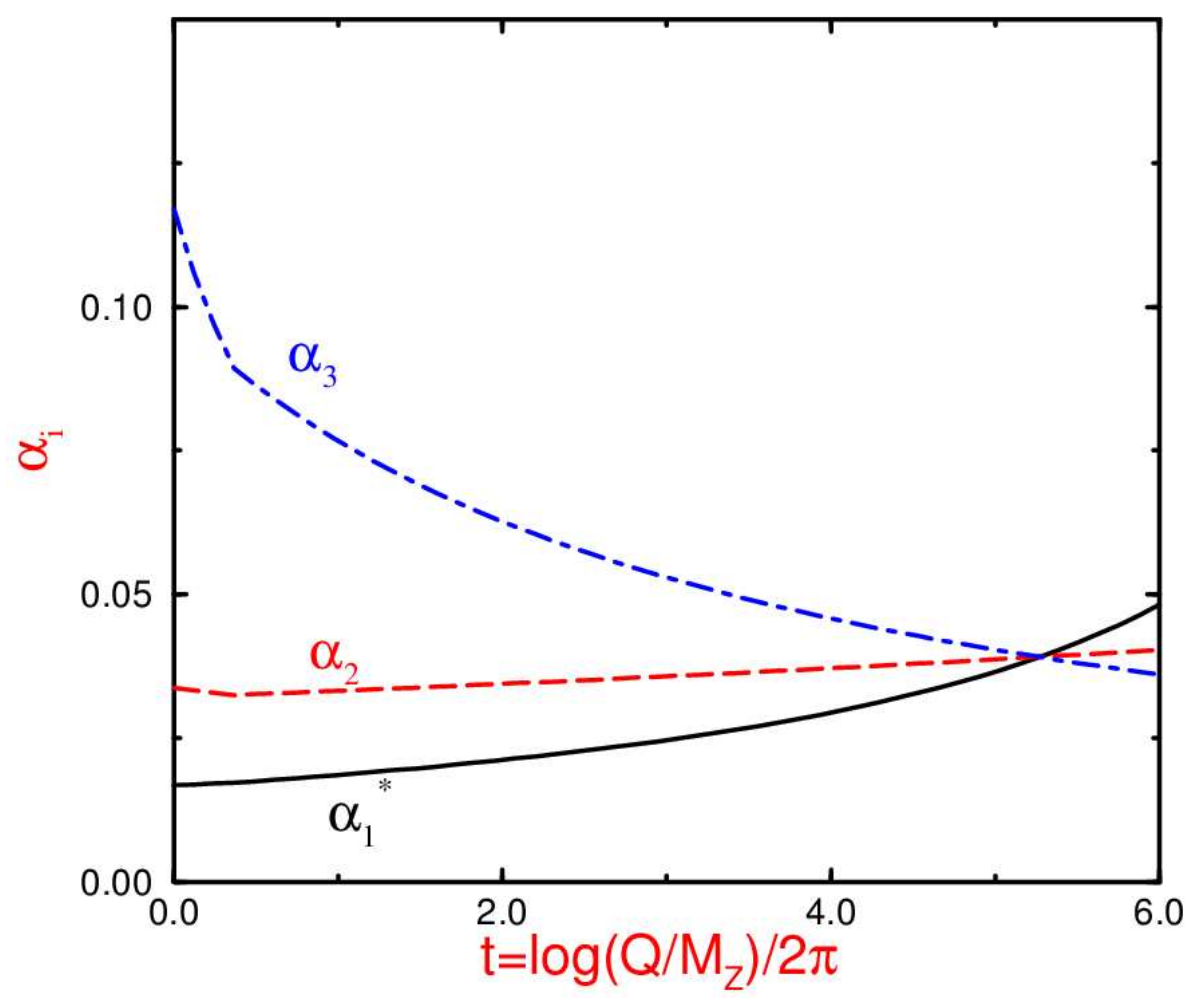

Figure 1.3: The evolution of the coupling strengths as a function of energy in supersymmetry, where $\alpha_{1}$ corresponds to $\mathrm{U}(1)$ (electromagnetic force), $\alpha_{2}$ corresponds to $\mathrm{SU}(2)$ (electroweak force), and $\alpha_{3}$ corresponds to $\mathrm{SU}(3)$ (strong force). Diagram from $[6]$.

Additionally, the Lightest Supersymmetric Particle (LSP) is stable and is a dark matter candidate. Heavy, stable, neutral particles that are predicted in supersymmetry could provide some of the missing mass of the universe which has been inferred to exist through measurements of large-scale gravitational effects [10]. Supersymmetry is thus a very intriguing extension to the Standard Model.

\subsection{Sparticle Spectrum}

The proposed spectrum of supersymmetric particles (sparticles) in mSUGRA (see Section 1.5) is shown in Table 1.4. Note that in supersymmetry there are multiple 


\begin{tabular}{|c|c||c|c|}
\hline Particle & Spin & Sparticle & Spin \\
\hline lepton & $1 / 2$ & slepton & 0 \\
\hline neutrino & $1 / 2$ & sneutrino & 0 \\
\hline quark & $1 / 2$ & squark & 0 \\
\hline gluon & 1 & gluino & $1 / 2$ \\
\hline W & 1 & chargino $(1,2)$ & $1 / 2$ \\
charged Higgs & 0 & & \\
\hline Z & 1 & & \\
$\gamma$ & 1 & & $1 / 2$ \\
light Higgs & 0 & neutralino $(1,2,3,4)$ & \\
heavy Higgs & 0 & & \\
pseudoscalar Higgs & 0 & & \\
\hline
\end{tabular}

Table 1.4: The particles of the Standard Model and their corresponding sparticles in supersymmetric models.

Higgs bosons (four in two doublets), and that the observable sparticles are formed as mixtures of the superpartners of the Standard Model particles. The superpartners are technically flavor eigenstates. There is one flavor eigenstate corresponding to each degree of freedom of the Standard Model particles. The observable spectrum of sparticles (the mass eigenstates) are linear combinations of the flavor eigenstates.

\section{5 mSUGRA}

Supersymmetry in its most general form has roughly 370 free parameters. The Standard Model without supersymmetry has about 30 free parameters. The number of free parameters in supersymmetry makes it impossible to make testable predictions based on the most general form of the theory. Theorists have worked on reducing the number of free parameters in supersymmetry by making assumptions.

The number of free parameters in supersymmetry can be reduced to about 100 by introducing a quantity called R-parity, which prevents rapid proton decay. R-parity is defined as:

$$
R=(-1)^{3(B-L)+2 S},
$$


where $B$ is the baryon number, $L$ is the lepton number, and $S$ is the spin of the particle.

The Standard Model particles have an R-parity of +1 , the sparticles have an Rparity of -1 , and the product of R-parity at all interaction vertices must be +1 . As a result of the introduction of R-parity, sparticles are produced in pairs, a sparticle decays to another sparticle plus a Standard Model particle, and the LSP is stable.

Other assumptions can be made to further reduce the dimension of the unknown parameter space. In the minimal supergravity (mSUGRA) framework, supersymmetry is broken at a very high energy scale. A theory that includes gravity operates at very high energies, and the broken supersymmetry is only an effective theory at low energies. The masses of the sparticles are functions of the interaction energy and are unified at the high energy scale.

In mSUGRA, there are only 5 free parameters which must be specified to make the theory completely predictable. The free parameters, set at some very high energy scale $\mathrm{M}_{\mathrm{x}}$, are:

- $\mathrm{m}_{0}$ : common mass for all of the scalar sparticles at the $\mathrm{M}_{\mathrm{x}}$ scale

- $\mathrm{m}_{1 / 2}$ : common mass for all of the gaugino sparticles at the $\mathrm{M}_{\mathrm{x}}$ scale

- $\mathrm{A}_{0}$ : parameter that describes the mixing of the left and right stop quarks at the $\mathrm{M}_{\mathrm{x}}$ scale

- $\tan \beta$ : ratio of the Higgs doublets' vacuum expectation values

- $\operatorname{sign}(\mu)$ : Higgsino mixing parameter.

The search for evidence of supersymmetry in this dissertation is done within the mSUGRA framework. 


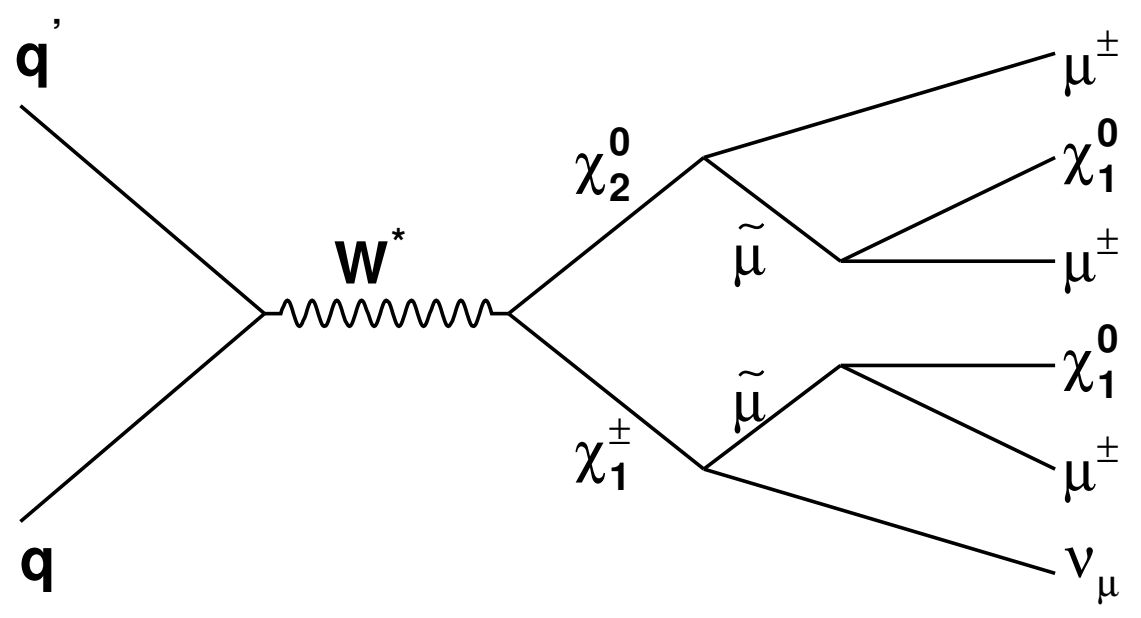

(a)

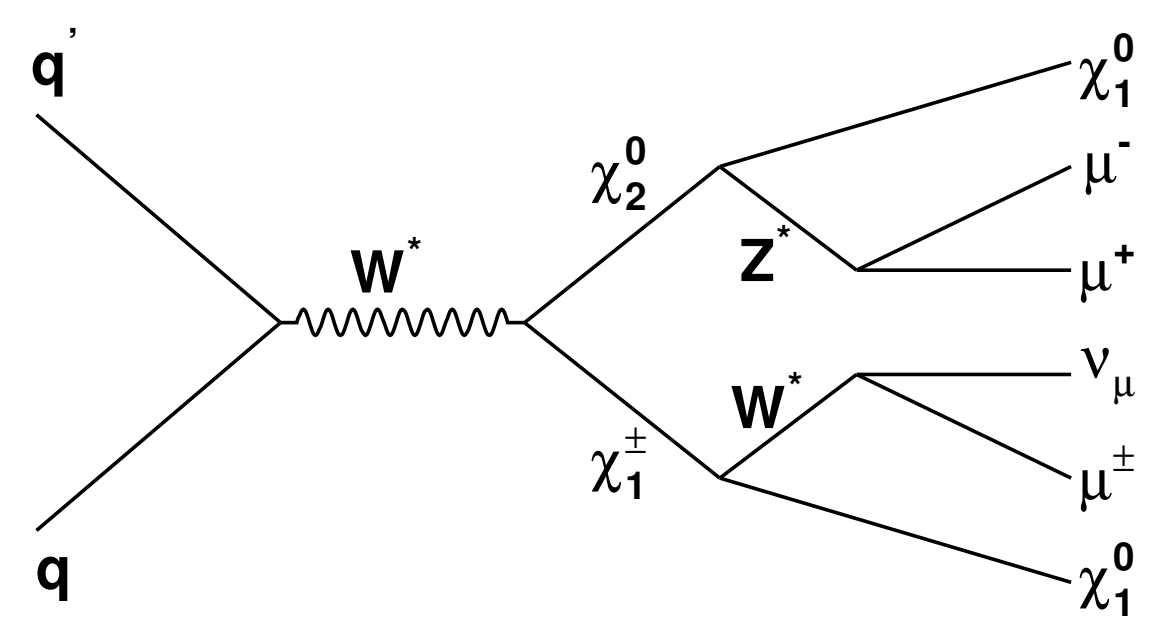

(b)

Figure 1.4: Feynman diagrams showing chargino $\left(\tilde{\chi}_{1}^{ \pm}\right)$and neutralino $\left(\tilde{\chi}_{2}^{0}\right)$ production and decay to three leptons and other particles including neutralinos $\left(\tilde{\chi}_{1}^{0}\right)$ through a) intermediate smuons $(\tilde{\mu})$ and b) intermediate virtual gauge bosons

\subsection{The Trilepton Final State}

Within the mSUGRA framework, calculations have been performed to predict signals which may be detectable using the $\mathrm{D} \varnothing$ detector (see Chapter 2). One such signal would occur with the associated production of chargino and neutralino sparticles (see Figure 1.4). These unstable sparticles will decay, with only stable LSPs leaving the 
detector. The decays of the chargino and neutralino can produce three leptons. Thus the final state would be three leptons plus missing energy from the LSPs. The trilepton final state has been called the "golden mode" for discovering supersymmetry because it is an uncommon final state in the Standard Model.

\subsection{Like-Sign Dimuon Channel}

In this dissertation, trilepton events are searched for by requiring pairs of leptons with the same sign of electric charge. This serves to increase the signal acceptance by requiring that only two out of the three leptons be detected. The requirement that the two leptons have the same sign of electric charge is introduced to remove the large background from opposite-sign lepton pairs. The background is created in the Drell-Yan process when a quark and antiquark annihilate through a virtual photon to produce opposite-sign lepton pairs. Other Standard Model sources of oppositesign lepton pairs include the decays of resonances such as the $J / \Psi, \Upsilon$, and $Z^{0}$. This dissertation is restricted to the study of the like-sign dimuon final state.

A key requirement for muons in this analysis is that they be isolated. Muons produced in association with jets (quarks) must be rejected, as muons from the signal should be produced in decays not involving jets. Muons produced with jets constitute a large background, mainly from the decay of B mesons. More on this background and others appears in Chapter 4. 


\section{Chapter 2}

\section{The Fermilab Accelerators and the DØ Detector}

Fermilab operates a series of accelerators in order to collide protons and antiprotons at a center-of-mass energy of $1.96 \mathrm{TeV}$ [11-13]. The final accelerator in the chain is the Tevatron, the world's highest energy particle accelerator. The D $\varnothing$ and CDF detectors surround the interaction regions where the collisions occur. The accelerators and the $\mathrm{D} \varnothing$ detector are described in the following sections.

The data used in this thesis were obtained during the data taking period known as Run II, which officially began in March, 2001. The previous data taking period, from 1992 to 1996, is known as Run I.

\subsection{The Fermilab Accelerators}

A chain of accelerators is necessary to produce and collide protons and antiprotons. The Cockroft-Walton preaccelerator, the linear accelerator (Linac), the Booster synchrotron, the Main Injector, the Antiproton Source, the Tevatron, and the Recycler work in series, with each using the particles passed to it from the accelerator preceding 


\section{FERMILAB'S ACCELERATOR CHAIN}

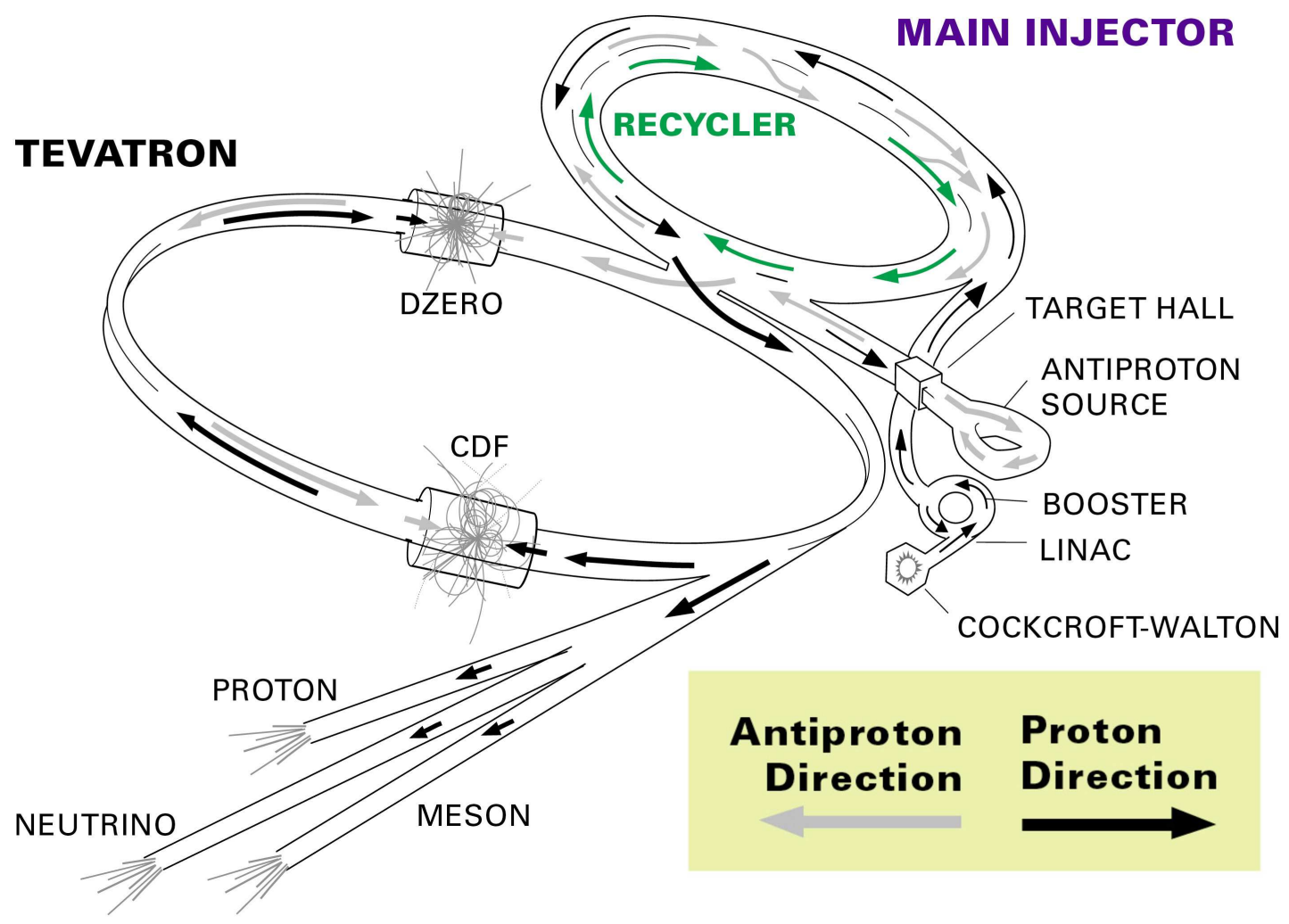

Figure 2.1: Schematic diagram of the Fermilab accelerator chain.

it. Figure 2.1 shows a schematic diagram of the Fermilab accelerator chain.

\subsubsection{The Preaccelerator}

The preaccelerator consists of an ion source, a Cockroft-Walton generator and accelerator column, and a transport line to the Linac. It is responsible for delivering negatively charged hydrogen ions with an energy of $18 \mathrm{keV}$. The source of the hydrogen ions is a sample of hydrogen gas.

An oval-shaped cathode surrounded by an anode sits in a magnetic field. Injection of the hydrogen gas into this environment creates a plasma. Charged particles randomly strike the cathode and sputter off hydrogen atoms which have been ab- 
sorbed on the surface. These negatively charged ions are accelerated to $750 \mathrm{keV}$ by a commercial Cockroft-Walton generator and accelerator column.

Once generated, the ions move into a transport line. Inside the transport line, a single gap radio frequency $(\mathrm{RF})$ cavity bunches the beam at the RF frequency of the Linac. The ions are then transferred to the Linac.

\subsubsection{The Linac}

The Linac accelerates the beam of ions to $400 \mathrm{MeV}$ using RF cavities. The Linac is a collection of steel tanks, each filled with a series of drift tubes separated by small gaps. The tubes are constructed such that a particle experiences accelerating fields when it is between drift tubes and is shielded from the decelerating fields while in the tubes. This requires the particle to pass through progressively longer tubes as it accelerates in order to maintain a constant phase with respect to the accelerating field. After acceleration, the ions are debunched and injected into the Booster.

\subsubsection{The Booster}

The Booster is a synchrotron which accelerates protons to $8 \mathrm{GeV}$. It consists of a series of 96 magnets interspersed with $17 \mathrm{RF}$ cavities in a circle with a radius of $75 \mathrm{~m}$.

Negatively charged hydrogen ions are transfered in bunches from the Linac to the Booster. The first bunch of ions passes through a thin carbon foil as it enters the Booster to remove the electrons from the ions. The next bunch of ions from the Linac can then be merged with the proton beam using a single magnetic field. The ions then have their electrons removed using the carbon foil. This is repeated with all of the ion bunches.

This technique is far better than attempting to accelerate protons in the Linac and 
consequently be required to add more protons to a proton beam already circulating in the Booster. That scheme would disturb the proton beam already circulating in the Booster.

The protons in the Booster are accelerated as the RF frequency of the Booster is increased. The magnets are ramped up synchronously to keep the particles in an orbit of constant radius. The protons are again bunched in this process. They are next passed to the Main Injector synchrotron.

\subsubsection{The Main Injector}

The Main Injector was built for Run II, to replace the Main Ring. The Main Ring, which accelerated protons for use in the Tevatron in Run I, was replaced by the Main Injector for several reasons.

First, because it was in the same tunnel as the Tevatron, the Main Ring passed through the region where the detectors were located. This required the construction of overpasses to carry the Main Ring beam over the experiments, which negatively affected the beam. Second, beam losses inside the D $\varnothing$ detector compromised detector data. Third, stray fields from the Tevatron disturbed the beam in the Main Ring.

To alleviate these problems, a new tunnel was built adjacent to the Tevatron to house the Main Injector. The Main Injector accelerates protons from $8 \mathrm{GeV}$ to $150 \mathrm{GeV}$ for insertion into the Tevatron, and accelerates protons to $120 \mathrm{GeV}$ for use in the Antiproton Source.

\subsubsection{The Antiproton Source}

The target used for production of antiprotons is a small nickel disk. Protons with $120 \mathrm{GeV}$ of energy strike the nickel disk target and produce a spray of particles. The spray contains antiprotons, which are selected using a dipole magnet. The antiprotons 
are directed to the Debuncher, where their momentum spread is reduced. They are then stored in the Accumulator. Once a large enough stack of antiprotons is available, it is transferred to the Main Injector and accelerated to $120 \mathrm{GeV}$.

\subsubsection{The Tevatron}

The Tevatron is the world's highest energy synchrotron accelerator. It is also the first large-scale superconducting synchrotron. All of its magnets are superconducting, cooled by liquid helium to a temperature of $4.6 \mathrm{~K}$. The Tevatron is approximately one kilometer in radius, and accelerates proton and antiproton beams from $150 \mathrm{GeV}$ to $980 \mathrm{GeV}$, for a center-of-mass energy in collisions of $1.96 \mathrm{TeV}$.

The proton and antiproton beams have a "bunch" structure due to the use of RF cavities in the acceleration of the particle beams. This means that collisions do not occur continuously inside the detectors, but at regular intervals. In Run II, the collisions occur every 396 ns. This collision rate is an important input into the design of the trigger and readout systems.

\subsubsection{The Recycler}

The Recycler is new for Run II. It was designed to increase the integrated luminosity (see Section 2.2.2) by providing for larger antiproton stacks in a shorter time. When the number of antiprotons in the antiproton beam drops below a usable level, they are transferred to the Recycler. Its purpose is to store the antiprotons for reuse.

During the data-taking period used for this analysis, the Recycler was not used.

\subsection{The DØ Detector}

The DØ detector [14-18] is comprised of several subdetectors (see Figure 2.2). Closest to the beamline is the magnetic central-tracking system. It consists of a silicon 


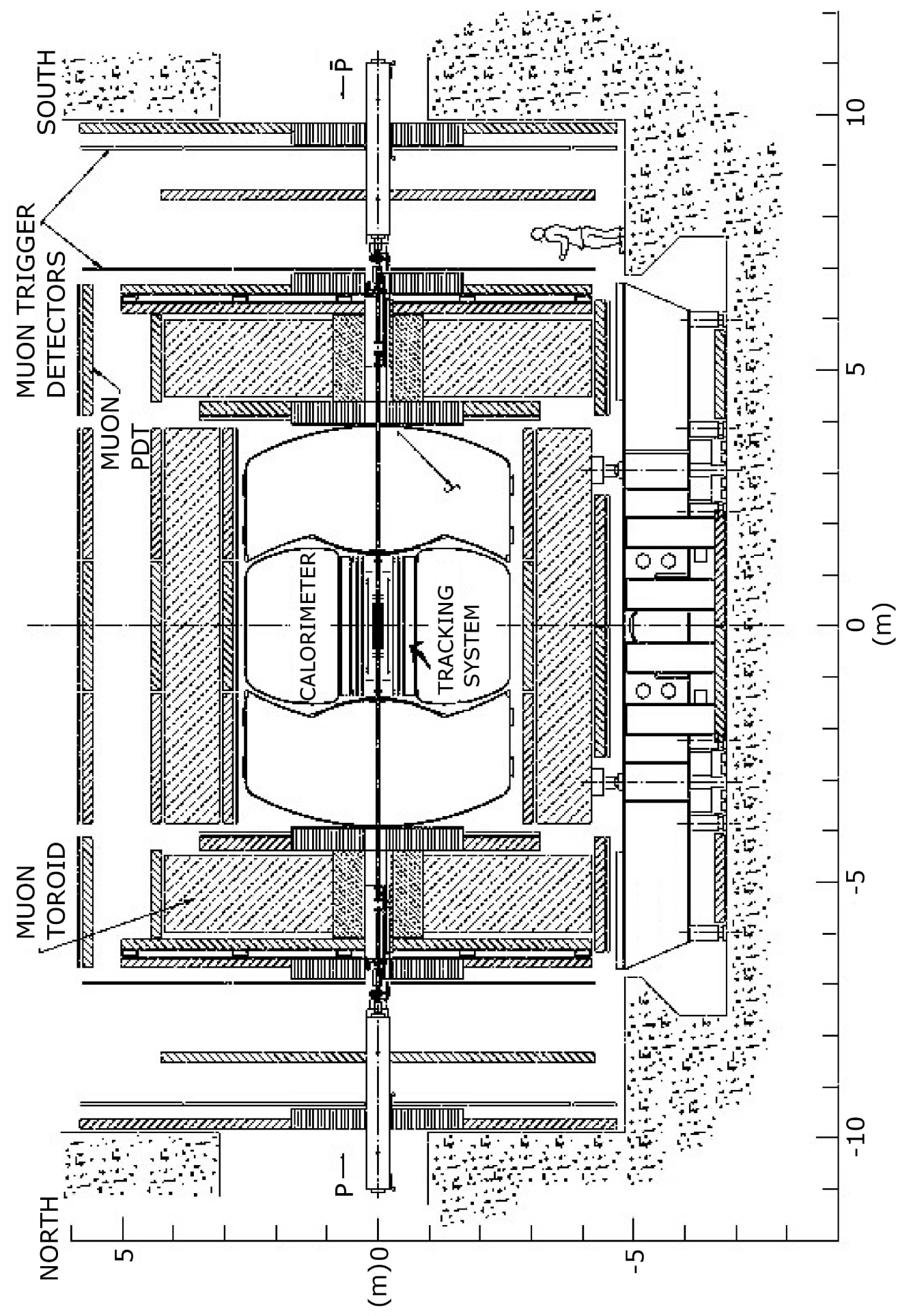

Figure 2.2: The DØ detector, including the tracking system, calorimeter, and muon system. 
microstrip tracker and a central fiber tracker, both located within a 2 T superconducting solenoidal magnet. The central and forward preshower detectors are located just outside of the superconducting coil (in front of the calorimeter), and are constructed of three layers of extruded triangular scintillator strips.

The next layer of detection includes three liquid-argon/uranium calorimeters: the central calorimeter (CC) and two end calorimeters (EC), all housed in separate cryostats. In addition to the preshower detectors, scintillators between the CC and EC cryostats provide sampling of developing showers at $0.8<|\eta|<1.4$.

A muon system resides beyond the calorimeter, and consists of a layer of tracking detectors and scintillation trigger counters, a layer of $1.8 \mathrm{~T}$ toroids, and two more layers of detectors after the toroids.

Luminosity is measured using two sets of 24 scintillation counters, located between the $\mathrm{CC}$ and the two EC cryostats. A forward-proton detector, situated in the Tevatron tunnel on either side of the interaction region, consists of a total of 18 Roman pots.

\subsubsection{Coordinate System}

The coordinate system for $\mathrm{D} \varnothing$ is right-handed, with the $z$-axis aligned along the beam direction, and $y$ pointing straight up. Positive $z$ is in the direction of proton travel. When spherical $(r, \phi, \theta)$ or cylindrical $(r, \phi, z)$ coordinates are used, $\phi=\pi / 2$ is along the positive y-axis, and $\theta=0$ is along the positive $z$-axis. Pseudorapidity $\eta$ is defined as

$$
\eta=-\ln \left(\tan \frac{\theta}{2}\right)
$$

and approximates the true rapidity

$$
y=\frac{1}{2} \ln \left(\tan \frac{E+p_{z}}{E-p_{z}}\right)
$$


in the limit that the invariant mass $m<<E\left(m^{2}=E^{2}-p^{2}\right)$.

Rapidity is a useful variable because Lorentz boosts in the $z$ direction (which are very common at a hadron collider) add only a constant to the rapidity of every particle in an event. Differences in rapidity between particles are thus unchanged under these boosts. Also, Lorentz boosts along the $z$ axis do not change the shape of rapidity distributions in an event.

\subsubsection{Luminosity}

Luminosity, a measure of particle flux, is a function of the number of particles passing through a unit area per unit time [16]. The luminosity determines the rate of interesting interactions, and much effort has been put into increasing the luminosity of the Tevatron.

The luminosity must be known in order to perform any analysis. It is measured at $\mathrm{D} \varnothing$ by detecting inelastic collisions, and the total amount of data collected over an extended period of time is expressed as the integrated luminosity over the whole time period. The luminosity group at $\mathrm{D} \varnothing$ provides tools to calculate the integrated luminosity of any data sample. DØ data are separated into luminosity blocks, typically about the amount of data taken in one minute. The luminosity group calculates the luminosity for each block, and declares any luminosity block which cannot have its luminosity calculated as bad. Bad luminosity blocks are not used in data analysis.

\section{Tevatron Time Structure and the Luminosity Calculation}

When determining the luminosity, the luminosity group at $\mathrm{D} \varnothing$ must take into account the Tevatron time structure [19].

The beams in the Tevatron are divided into bunches. A bunch is a group of particles revolving together in the Tevatron. Each revolution (also known as a turn) takes approximately $21 \mu \mathrm{s}$. There are 1113 distinct RF buckets. Each RF bucket is 
separated by approximately 18.8 ns. A tick is defined as a grouping of seven RF buckets, with the bunch located in the first bucket, and the remaining six buckets empty. Each tick is separated by approximately $132 \mathrm{~ns}$.

There are 159 ticks in one turn. The Tevatron currently runs in a mode where 36 bunches of protons and 36 bunches of antiprotons are in the machine at one time. The 36 bunches are separated into 3 symmetrically spaced superbunches, with 19 empty ticks between the last bunch in one superbunch, and the first bunch in the next superbunch. There are two empty ticks between each bunch inside a superbunch, and therefore collisions occur every 396 ns inside a superbunch.

The luminosity system's scintillation counters collect data and increment scalers for each tick separately. This is necessary because the characteristics of each bunch may differ, changing the average number of interactions per crossing. The luminosity calculation includes a sum over all of the ticks.

\subsubsection{Silicon Microstrip Tracker}

The innermost detector system in the $\mathrm{D} \varnothing$ detector is the silicon microstrip tracker (SMT) [20]. It is part of the inner tracking system (see Figure 2.3), which is designed to measure momenta of charged particles bending within the magnetic field of a $2 \mathrm{~T}$ superconducting solenoidal magnet. The SMT also measures the positions of primary and secondary vertices, which are the locations of partonic collisions in the event. The SMT is the high-resolution part of the tracking system, essential in b quark identification through precise measurement of secondary vertex positions. For example, b quarks are produced in top quark decays, and give rise to B mesons which travel a short distance before decaying. The location of this decay is measurable as a secondary vertex, and is evidence of the presence of a b quark in the event.

The SMT surrounds the interaction region. Beam collisions are most likely to occur at the center of the detector, however, the typical width of the interaction 


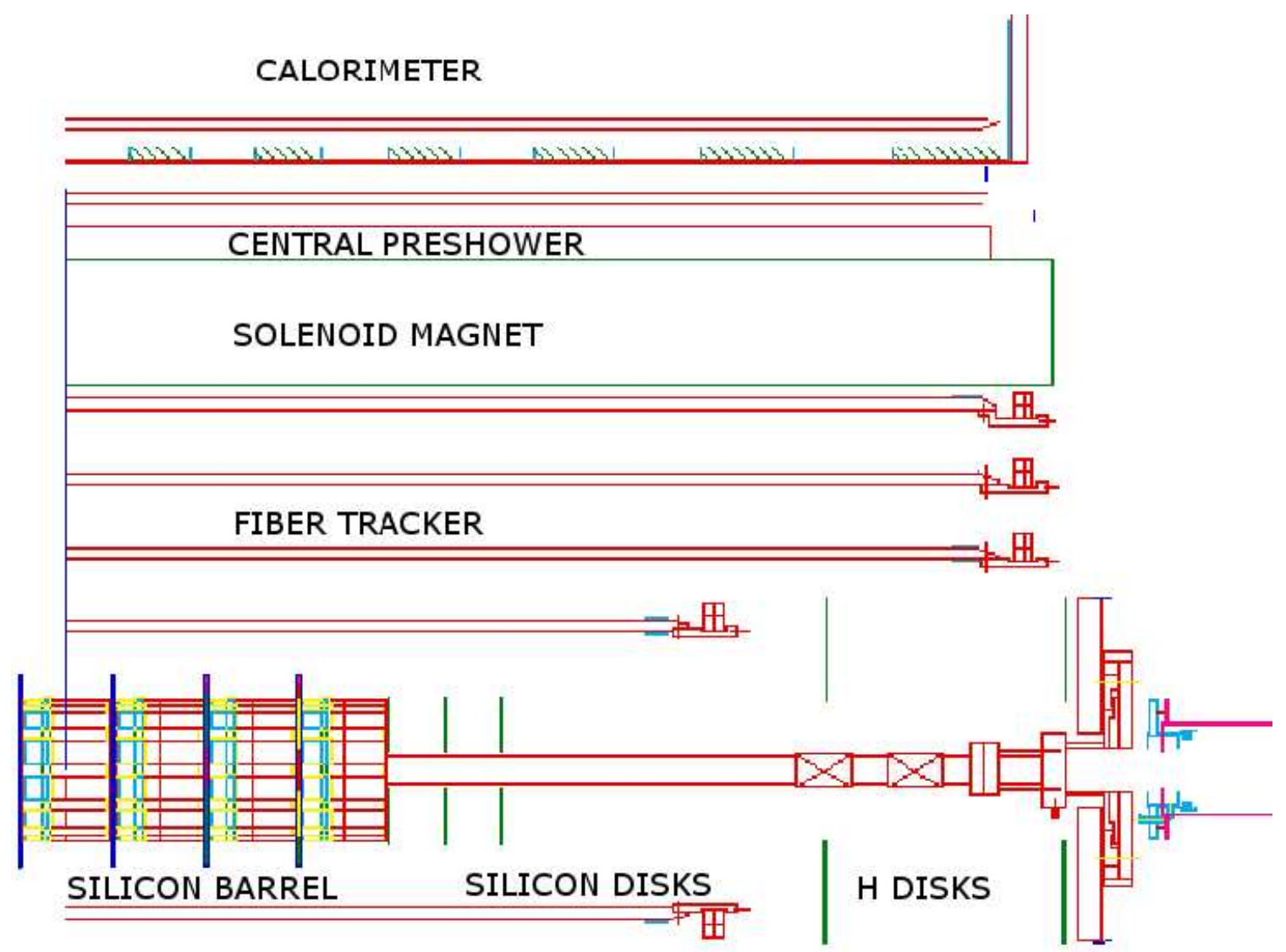

Figure 2.3: The D $\varnothing$ tracking system surrounding the beam pipe, including the silicon barrels and disks and the fiber tracking.

"point" extends $25 \mathrm{~cm}$ in the $z$ direction. To detect charged particles emanating from a collision not at the center of the detector, the SMT has a hybrid design.

It is difficult to build the SMT such that all particles' tracks are perpendicular to the detector, due to the extended interaction point. The solution is to build barrel detectors to measure primarily the $r-\phi$ coordinate, and disk detectors to measure $r-z$ as well as $r-\phi$ (see Figure 2.4). At high values of $\eta$, vertices for particles are reconstructed in three dimensions by the disks, and at small $\eta$ the vertices are determined by the barrels. The detector has six barrels along the $z$-axis, with four detector layers per barrel. There are twelve small diameter double-sided "F" disks (Figure 2.5), and four large diameter single-sided "H" disks. Four F disks are placed between barrels, except at $\eta=0$. The remaining eight are placed at the ends of the 


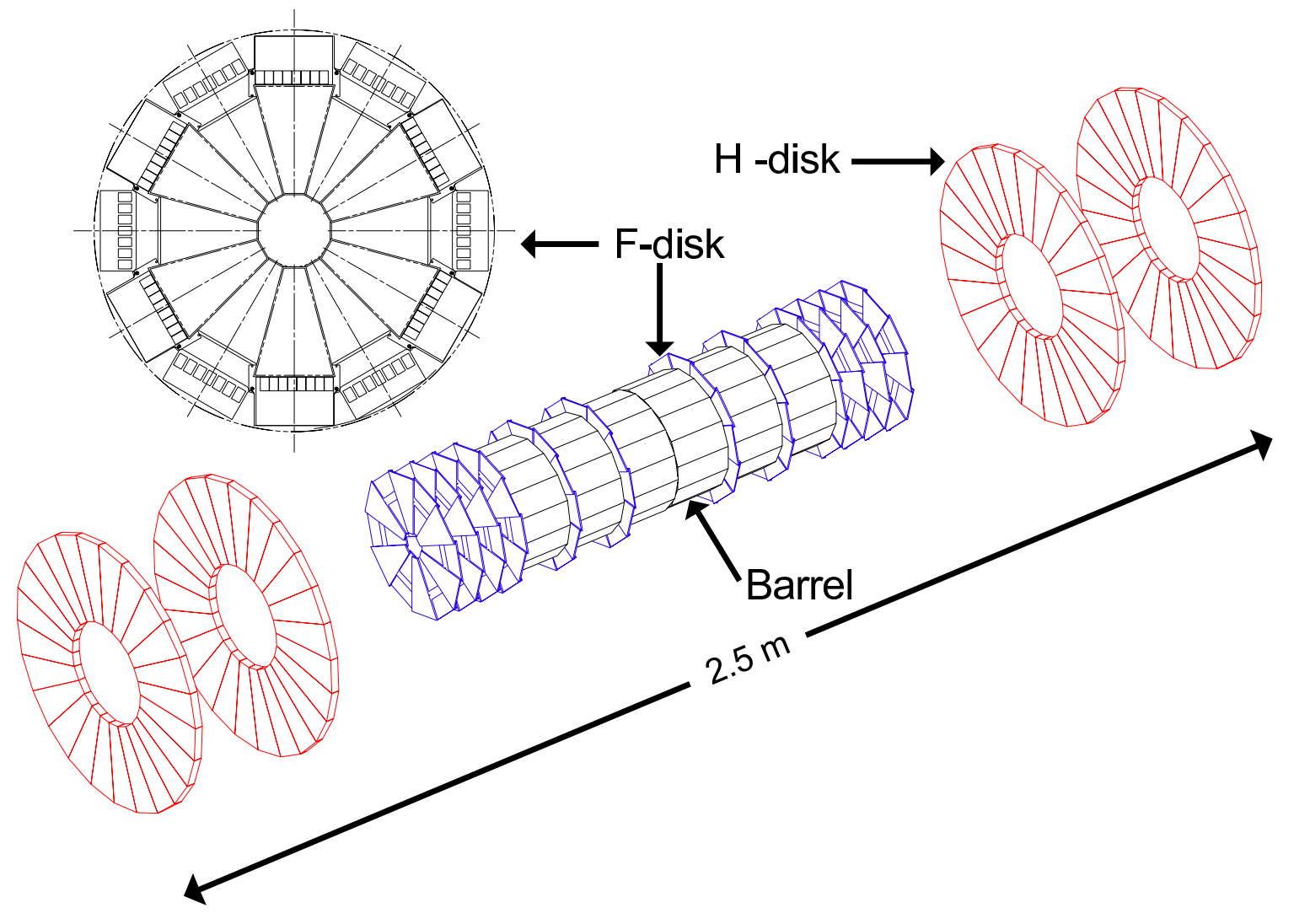

Figure 2.4: Diagram of the DØ silicon barrel detector.

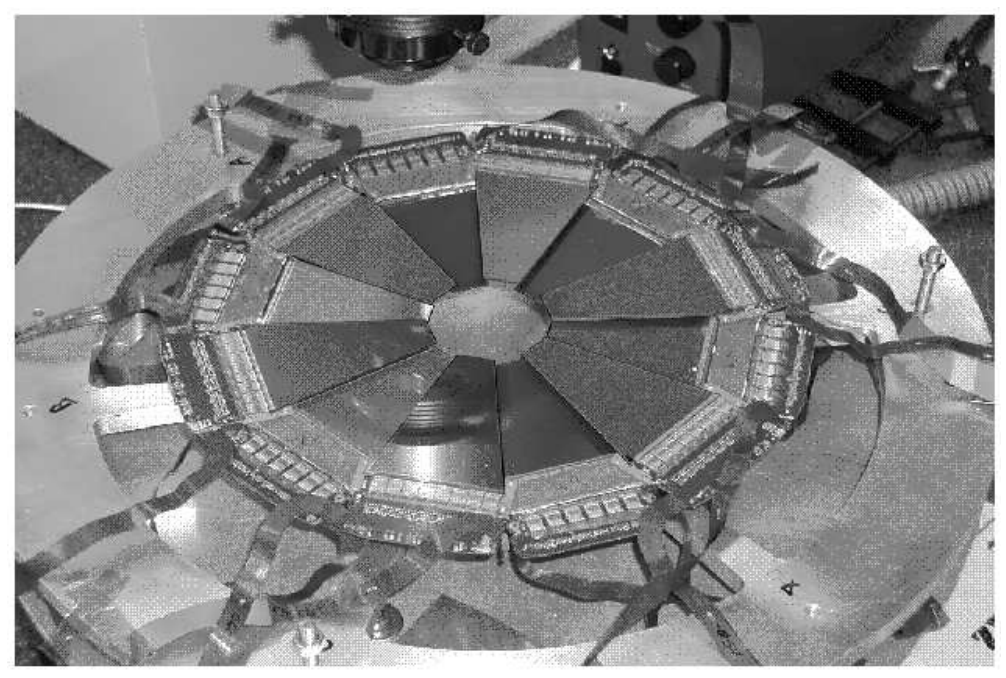

Figure 2.5: An F disk, part of the $\mathrm{D} \varnothing$ silicon detector. Each of the 12 individual wedges is approximately $7.5 \mathrm{~cm}$ in length.

barrels. The $\mathrm{H}$ disks sit at the furthest distance from $z=0$, approximately $1.2 \mathrm{~m}$ away, and allow coverage out to $\eta \approx 3$. During the data-taking period used for this 


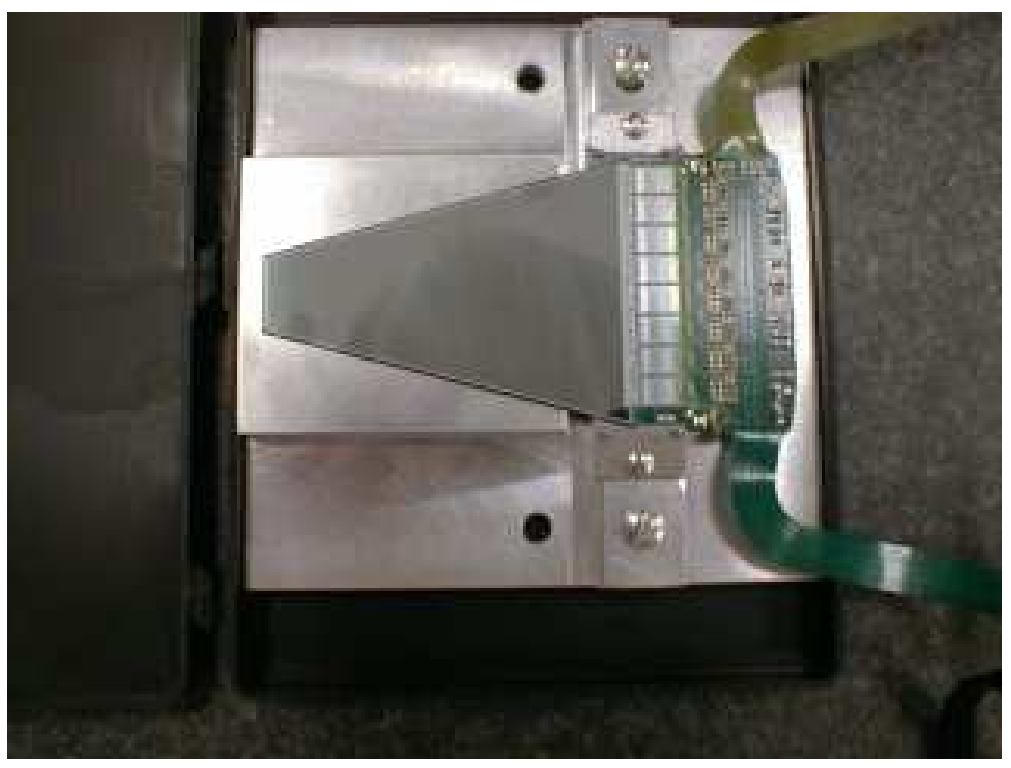

Figure 2.6: Photograph of wedge used in F disk in the D $\varnothing$ silicon detector. The wedge is approximately $7.5 \mathrm{~cm}$ in length. Also visible are the SVX II chips used to read out the detector.

analysis, the $\mathrm{H}$ disks were not used.

The barrels and F disks are composed of $300 \mu \mathrm{m}$-thick silicon microstrip detectors (Figure 2.6). In all, the SMT has approximately 800,000 individual strips. This allows a spatial resolution of approximately $10 \mu \mathrm{m}$, and the pattern recognition necessary to reconstruct tracks inside jets.

\subsubsection{The Scintillating Fiber Tracker}

The scintillating fiber tracker, or central fiber tracker (see Figures 2.9 and 2.10), is the next detector that most particles encounter as they move outward from the interaction point [21]. The detector covers the region $|\eta|<2$, and is used in conjunction with the SMT to measure trajectories and momenta of charged particles with excellent resolution (see Figure 2.8). The tracker also provides information for the triggering system.

The scintillating fiber tracker consists of approximately 70,000 scintillating fibers mounted on eight concentric cylinders at radii of 19.5, 23.4, 28.1, 32.8, 37.5, 42.1, 


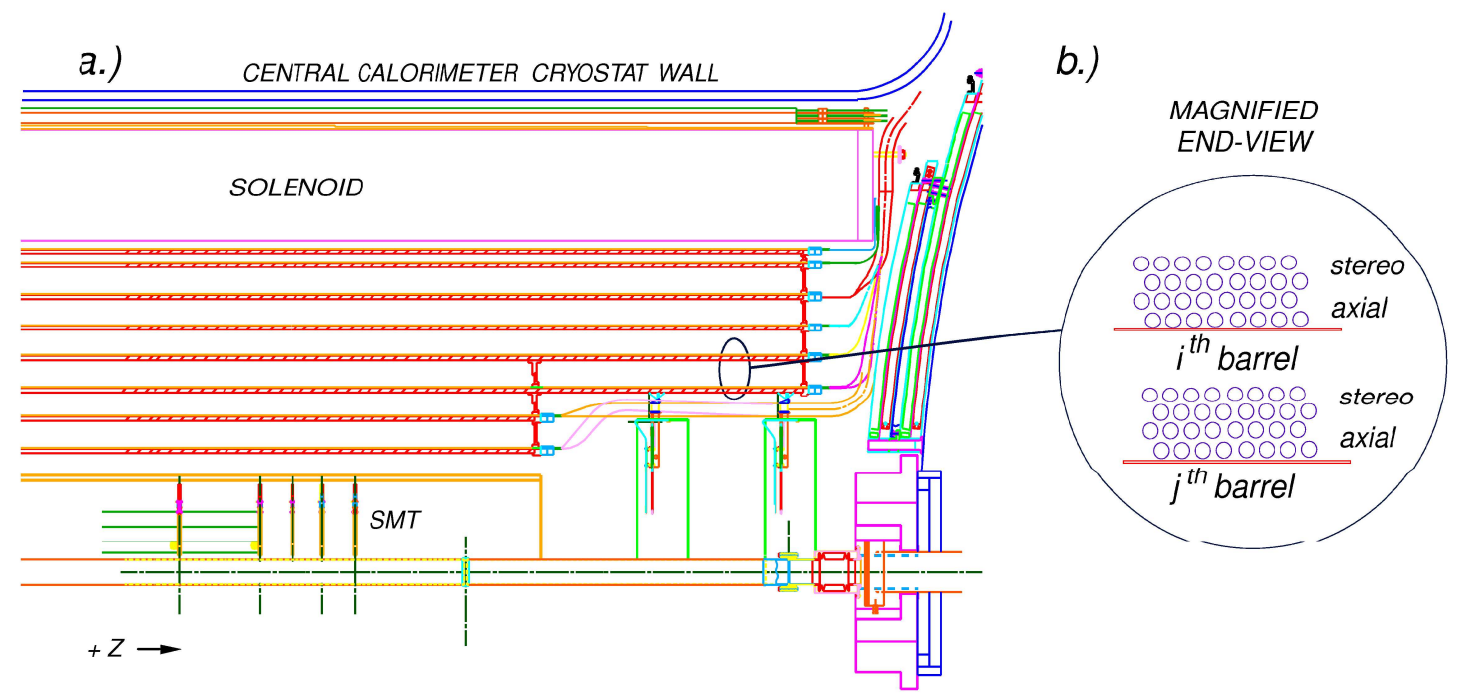

Figure 2.7: a) Diagram showing the location of the D $\varnothing$ central fiber tracker. b) Closeup view showing the axial and stereo layers of scintillating fibers attached to detector barrels.

48.8, and $51.5 \mathrm{~cm}$ (Figure 2.7). Each cylinder supports two doublets of overlapping scintillating fibers of $0.835 \mathrm{~mm}$ diameter, one doublet parallel to the collision axis, and the other alternating by $\pm 3^{\circ}$ relative to the axis. The clear fibers have an inner polystyrene core (index of refraction 1.59) surrounded by a thin acrylic cladding (index of refraction 1.49), and a thin fluoro-acrylic cladding (index of refraction 1.42). The second cladding exists to increase the light trapping efficiency. The fibers scintillate in the yellow-green part of the visible light spectrum.

The fibers must be precisely mounted onto the mechanical structure of the fiber tracker in order to accurately reconstruct tracks. Perhaps more importantly, triggering will be adversely affected by fibers not mounted parallel to the beam axis. The other important issue entering into the construction of the fiber tracker is the thickness of the support cylinders. They must be as thin as possible to minimize multiple scattering. Both of these issues were considered during the design of the fiber tracker [21].

Scintillation light is transferred via clear multiclad waveguides to solid-state, vis- 


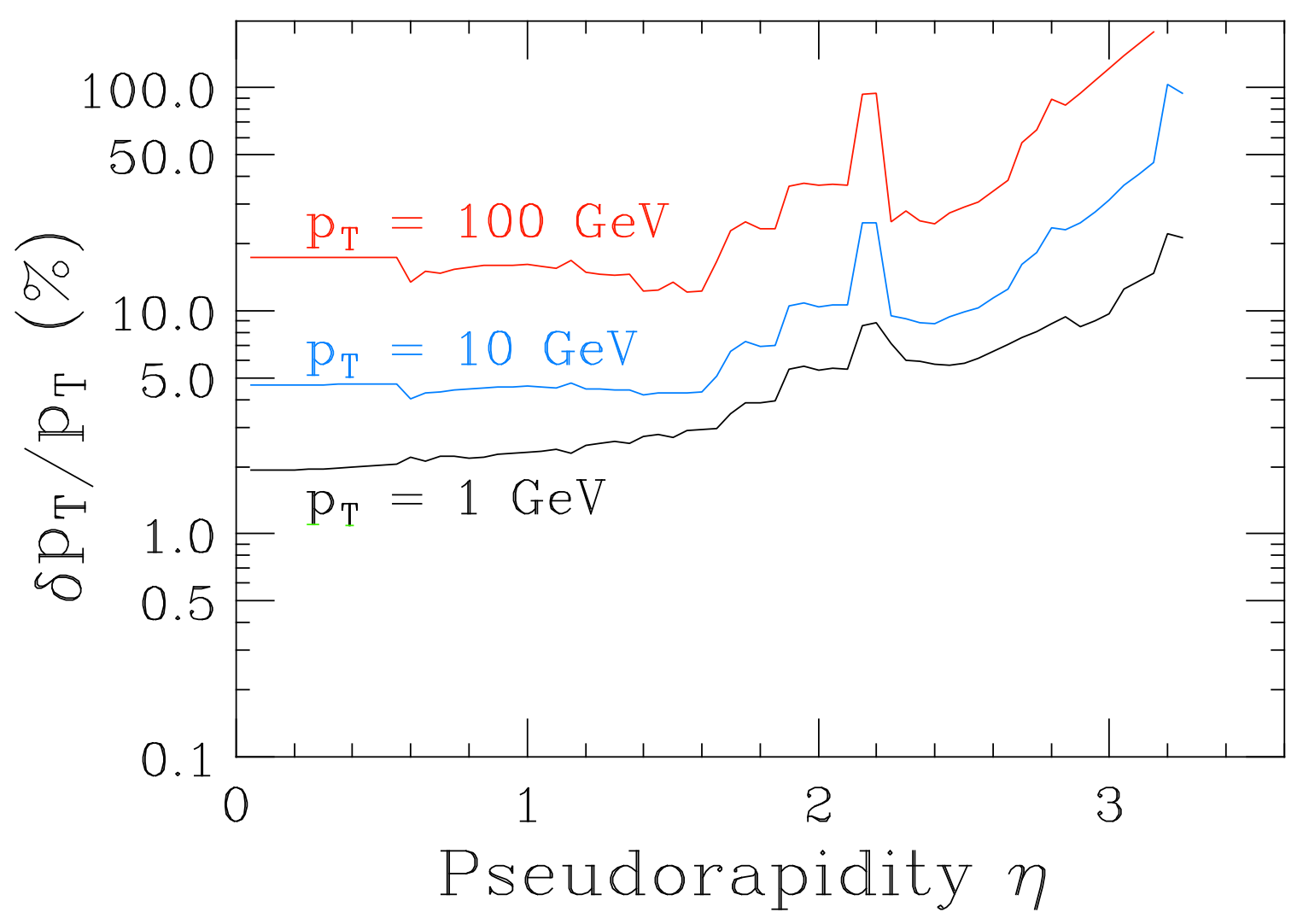

Figure 2.8: Simulation of the transverse momentum $\left(\mathrm{p}_{\mathrm{T}}\right)$ resolution of the tracking system as a function of pseudorapidity for tracks with $\mathrm{p}_{\mathrm{T}}=1,10$, and $100 \mathrm{GeV} / \mathrm{c}$.

ible light photon counters (VLPC) that have approximately 80\% quantum efficiency, and a gain of at least 20,000. The VLPC operate at around $10 \mathrm{~K}$ to reduce the background from electronic noise, and have a rate capability of at least $10 \mathrm{MHz}$.

\subsubsection{Preshower Detectors}

The central and forward preshower (CPS and FPS) detectors are located just outside of the superconducting coil and inside of the calorimeter $[22,23]$. These detectors each consist of a tapered lead radiator and three (CPS) or four (FPS) layers of triangular scintillator strips that are read out using wavelength-shifting fibers and VLPCs (see Figure 2.11). The preshower detectors aid in electron identification and triggering and correct electromagnetic energy measurements for solenoid effects. The detectors can 


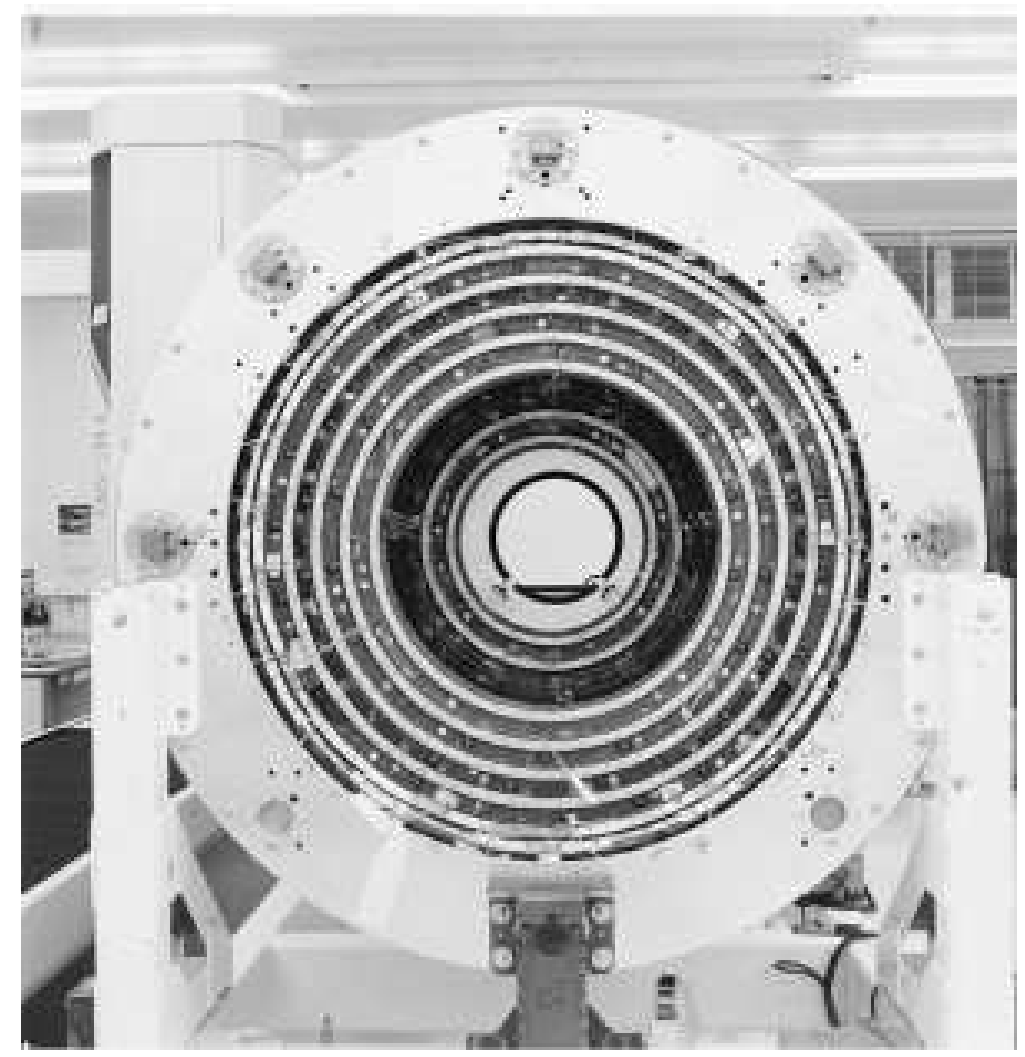

Figure 2.9: End view of the $\mathrm{D} \varnothing$ central fiber tracker.

be used to accurately measure positions and sample energies of particles before they enter the calorimeter.

The CPS detector (see Figure 2.14) fits in the small (51 mm) gap between the solenoid coil and the central calorimeter cryostat. It is a cylindrical detector with a radius of approximately $72 \mathrm{~cm}$, covering the region $|\eta|<1.2$. Two FPS detectors cover the regions $1.4<|\eta|<2.5$. The FPS detectors are mounted to the inner faces of each of the end calorimeter cryostats.

Electrons and photons traversing the detectors will have already passed through the solenoid and tapered lead radiator. Most of these particles will begin electromagnetic showers in those materials (discussed in Section 2.2.6), and therefore deposit a large amount of energy in the preshower detector. Muons and charged pions will leave only a small amount of energy in the detector through ionization. Charged pions will, 


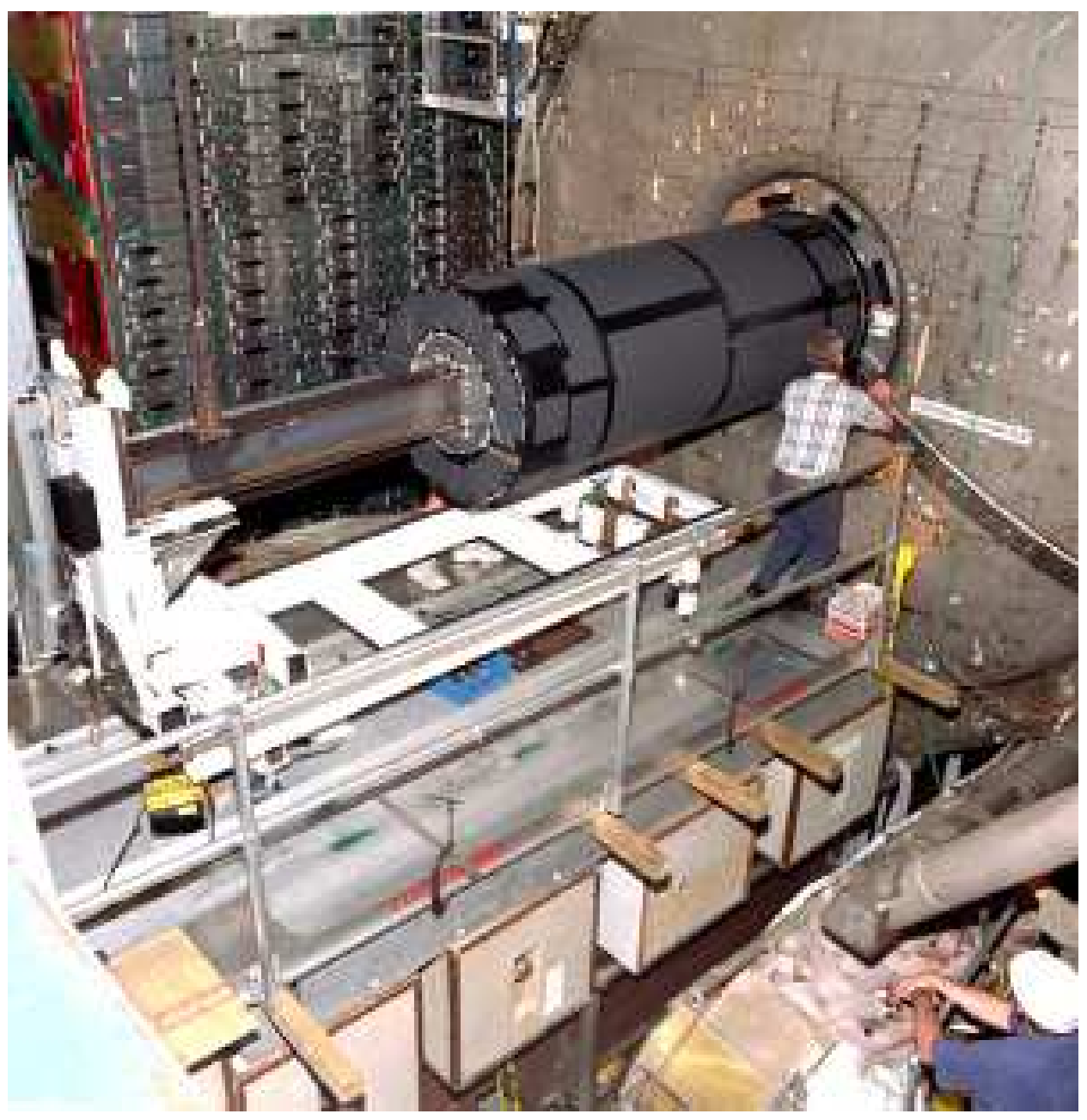

Figure 2.10: Insertion of the central fiber tracker into the D $\varnothing$ detector, inside the calorimeter.

however, often begin hadronic showers in the solenoid. These showers are the biggest source of background for electron identification in the preshower detector.

The tapered lead radiator is used to ensure that the total amount of material is constant at all pseudorapidities. Figures 2.12 and 2.13 show the amount of material needed to have a constant two radiation lengths of material before the CPS detector.

\subsubsection{Calorimeter}

DØ has three uranium liquid-argon calorimeters - a central calorimeter (CC) and two end calorimeters (EC) - which are usually referred to collectively as the calorimeter 
a.)

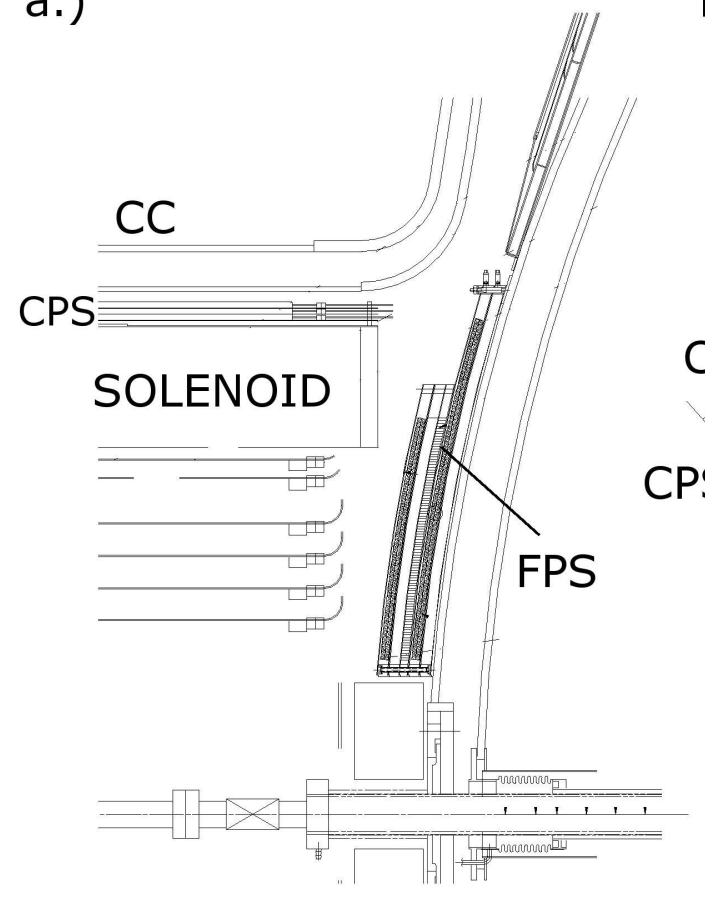

b.)

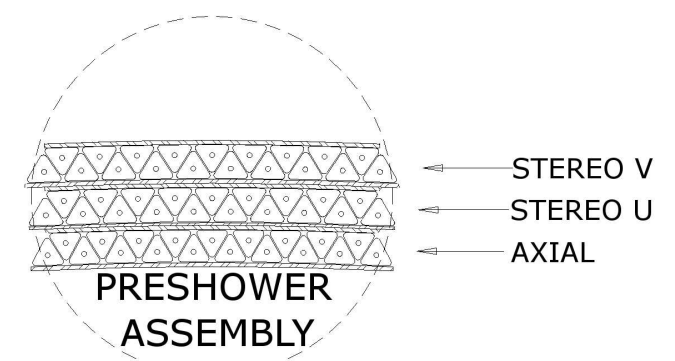

Figure 2.11: a) Diagram showing the locations of the central (CPS) and forward (FPS) preshower detectors, outside the central fiber tracker (CFT). b) End view of the CPS, including a closeup view of the three layers of scintillator strips. Not drawn is the tapered lead radiator, which is between the solenoid and the preshower detector.

(Figures 2.15 and 2.16). The CC covers the $|\eta|<1.2$ range, while the two EC on either side cover the regions $1.3<|\eta|<4$.

The calorimeter is used in the identification of electrons, photons, jets, and muons, and in the measurement of the missing transverse energy in an event. It is responsible for the energy measurement of electrons, photons, and jets.

The calorimeter is composed of a large number of modules, each of which consists of a stack of interleaved absorber plates and signal boards (cells). The modules are organized into layers and differ mainly by the type of absorber used. The electromagnetic modules use depleted uranium, the fine hadronic modules use depleted uranium with $1.7 \%$ niobium, and the coarse hadronic modules use copper or stainless steel. See Figure 2.17 for a schematic view of a calorimeter cell. Liquid argon fills 


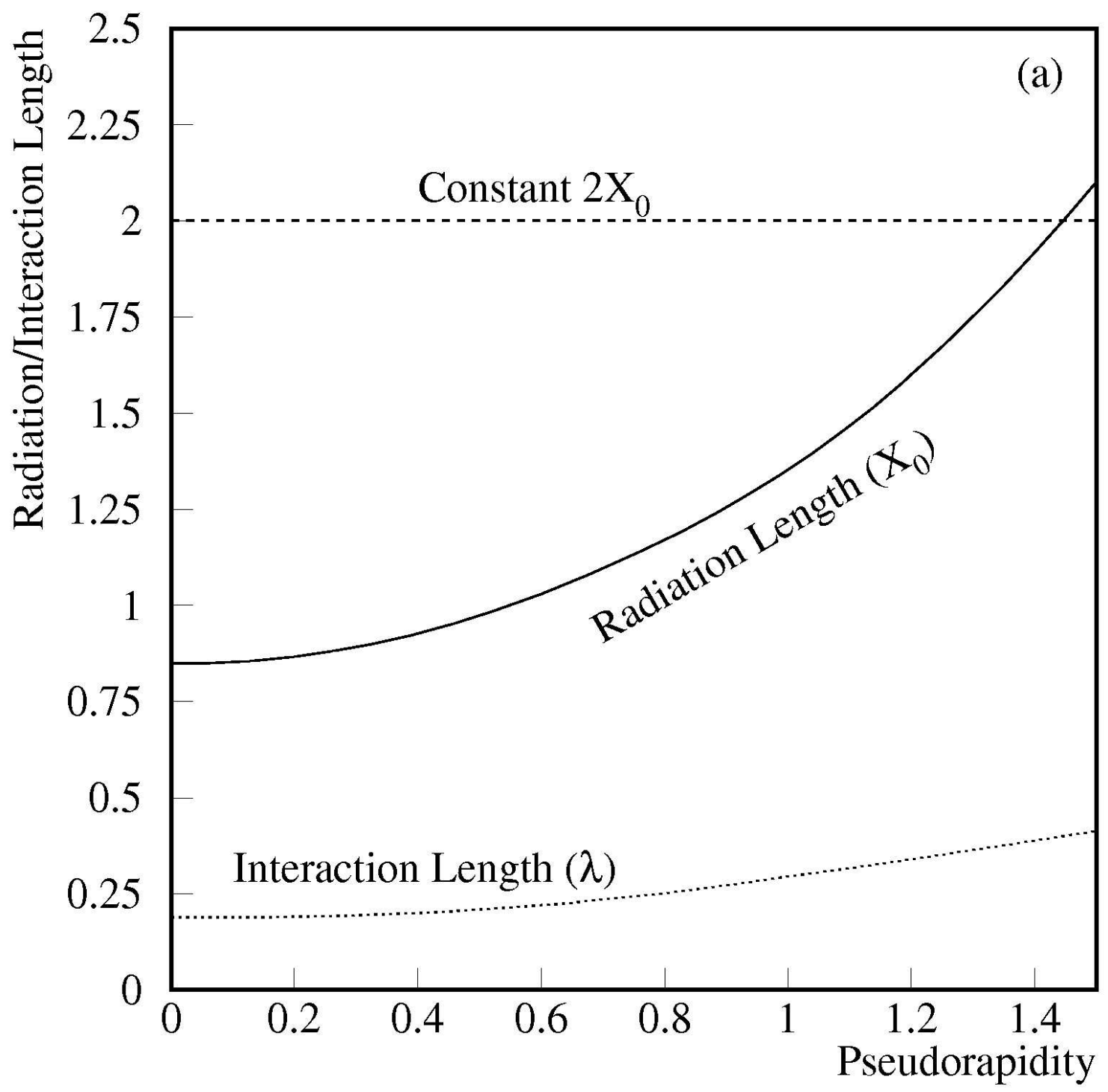

Figure 2.12: The solenoid's radiation and nuclear interaction lengths as functions of pseudorapidity. Diagram from [22].

the $2.3 \mathrm{~mm}$ gap between the absorber plates and signal boards. The calorimeter sits inside a cryostat in order to keep the argon cold.

Liquid argon is used because it is dense, radiation-hard, and reliable, and provides a uniform, linear response. It allows for relatively easy calibration and fine segmentation.

Uranium is used because of its high density, allowing a more compact calorimeter. 


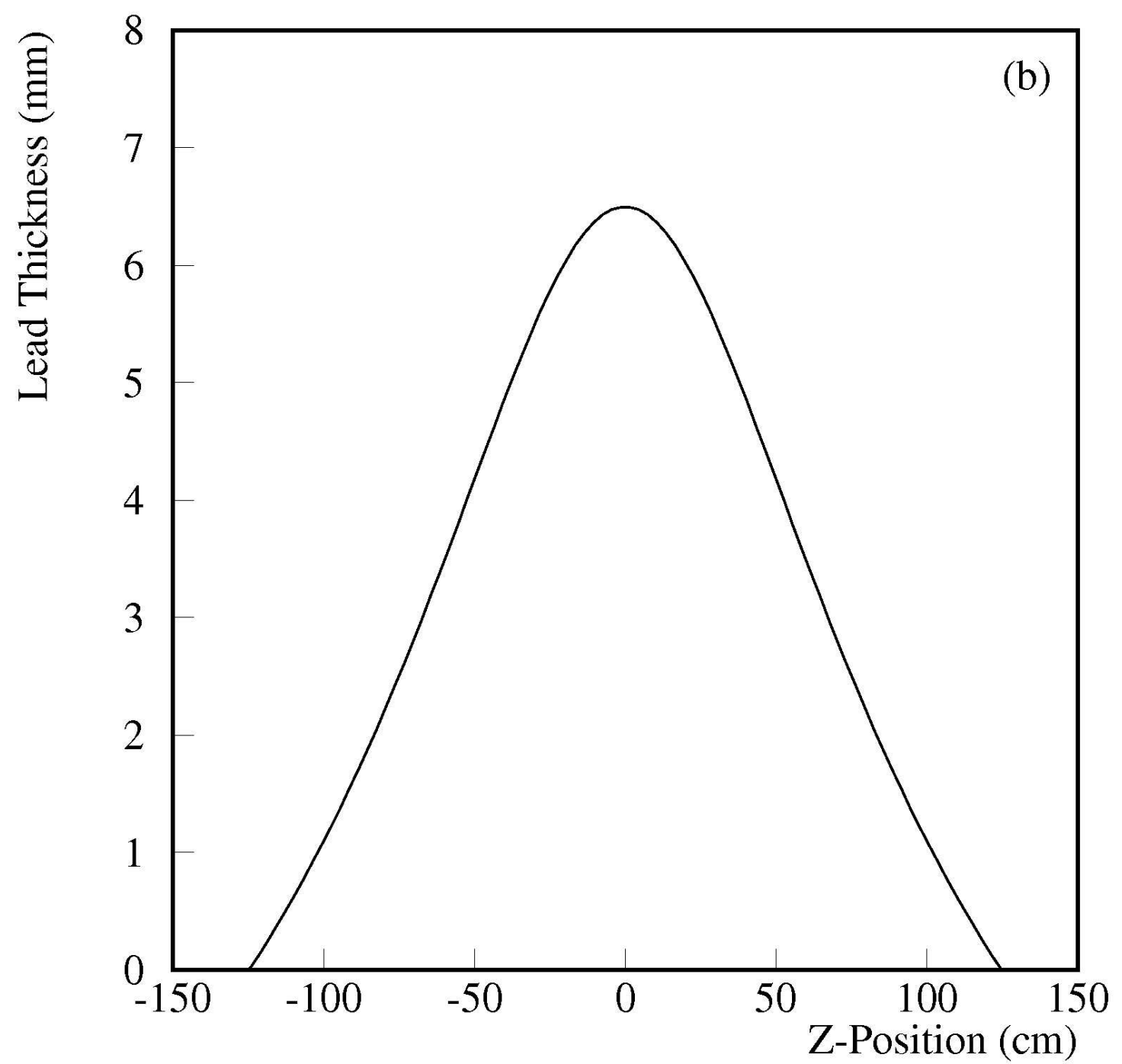

Figure 2.13: Thickness of lead needed to yield a constant $2 \mathrm{X}_{0}$ (2 radiation lengths) for all particle trajectories. Diagram from [22].

It also improves the energy measurement because energy losses in the uranium are compensated by energy released in fission of ${ }^{238} \mathrm{U}$.

Electrons entering the calorimeter interact with the absorber plates through the Bremsstrahlung mechanism, in which they emit a photon upon feeling the Coulomb field of a nucleus in the absorber. Photons interact predominantly via pair production, when a photon converts into an electron-positron pair in the vicinity of a nucleus. The secondary particles emitted in these interactions can undergo these same in- 


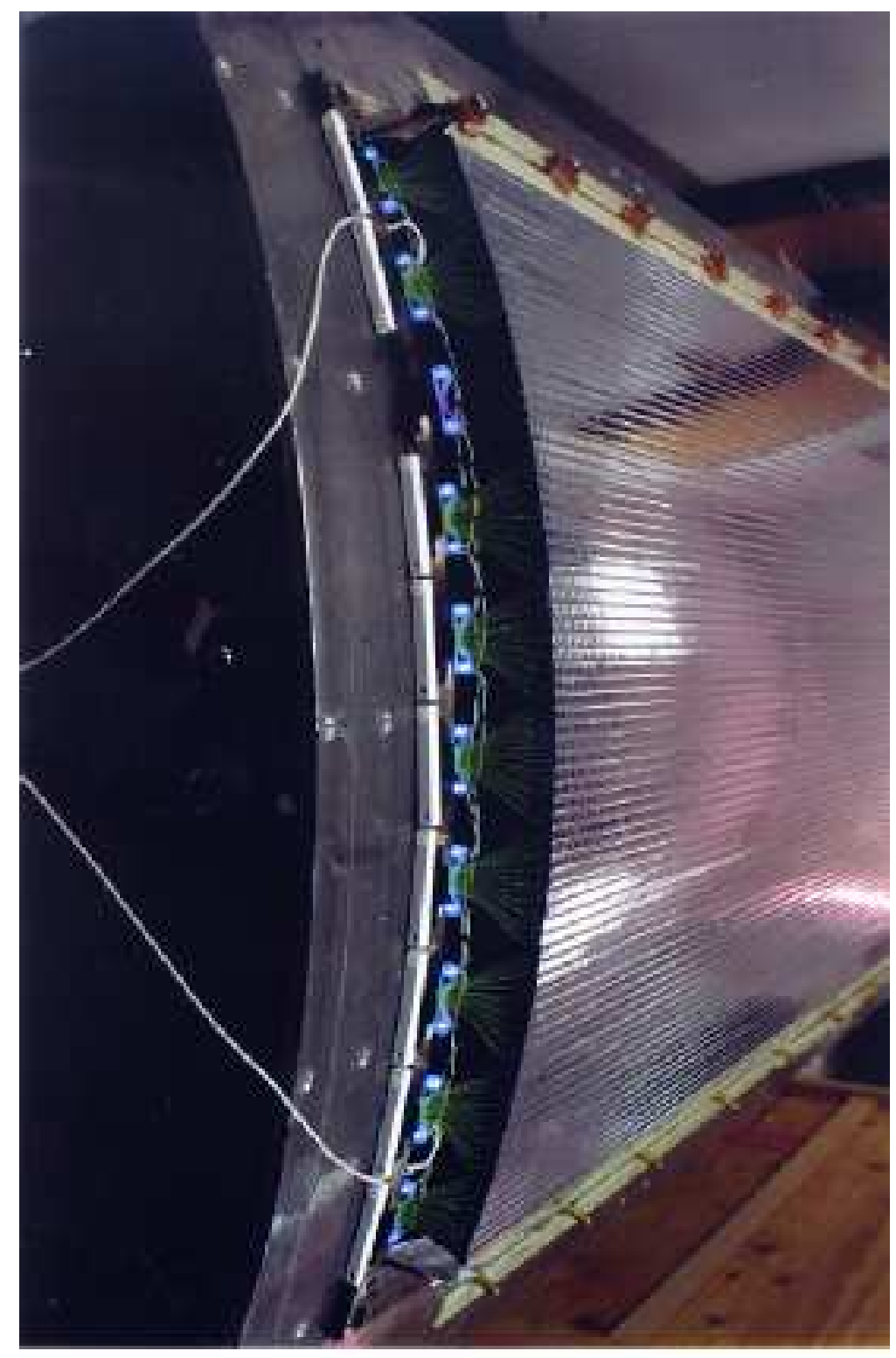

Figure 2.14: A portion of the $\mathrm{D} \varnothing$ central preshower detector, a thin cylindrical detector mainly consisting of scintillating fibers.

teractions themselves. This process, called an electromagnetic shower, repeats itself until the energies of all the secondary particles fall below the threshold for pair production. The particles will then continue to lose energy, mainly through ionization. The calorimeter works by measuring the energy released in this shower, which is an indirect measurement of the energy of the original particle.

Hadronic particles lose energy in the calorimeter mainly through inelastic collisions 


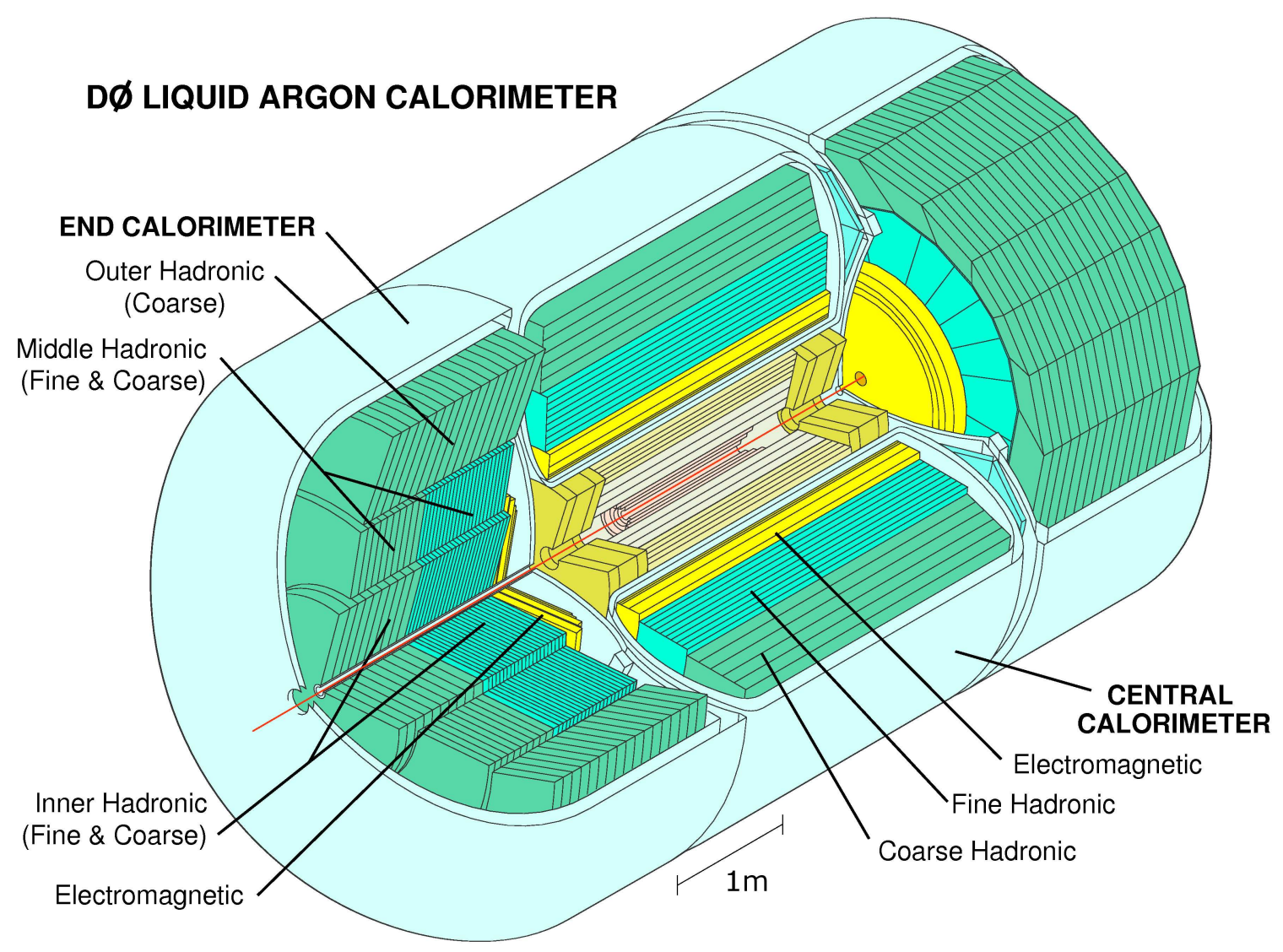

Figure 2.15: The D $\varnothing$ calorimeter, composed of layers of heavy metals and liquid argon.

with atomic nuclei. The secondary particles produced in these collisions can also lose energy through inelastic collisions. This process is called a hadronic shower. Hadronic showers are more extended in space than electromagnetic showers.

The $\mathrm{D} \varnothing$ calorimeter is a sampling calorimeter because it uses layers of absorber plates to absorb most of the energy of the incident particles while causing them to shower, and its liquid argon sections to sample the energy of the showers. This sampling is done by grounding the absorber plates and maintaining the copper pads on the signal boards at a voltage of approximately $2 \mathrm{kV}$. Charged particles passing through the liquid argon ionize electrons in the argon. These ionized electrons are drawn across the potential difference to the signal boards and they induce a signal on the copper pad. These signals allow measurement of the energy in the showers.

The final pieces of the calorimeter are between the CC and EC. Particles entering 


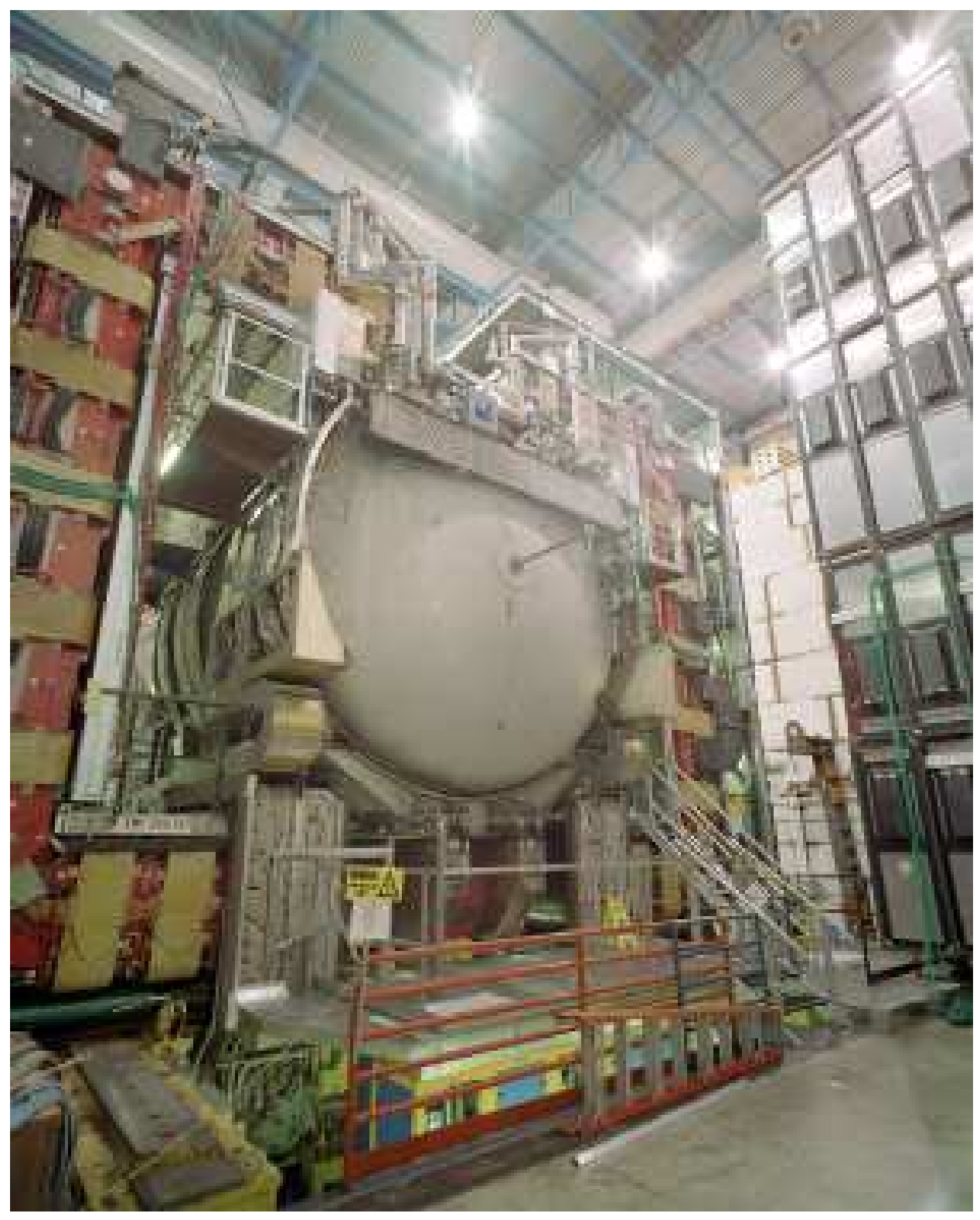

Figure 2.16: The $\mathrm{D} \varnothing$ calorimeter sitting inside the $\mathrm{D} \varnothing$ detector outside of the collision hall during the period between Run I and Run II.

this region may escape detection by traveling through the space between the $\mathrm{CC}$ and EC. To measure energies of particles in this region, massless gaps (MG) and the intercryostat detector (ICD) are used. The MG are rings of signal boards mounted on the ends of parts of the CC and EC. The ICD is a ring of scintillation counters mounted on the exterior of the EC cryostats [16]. 


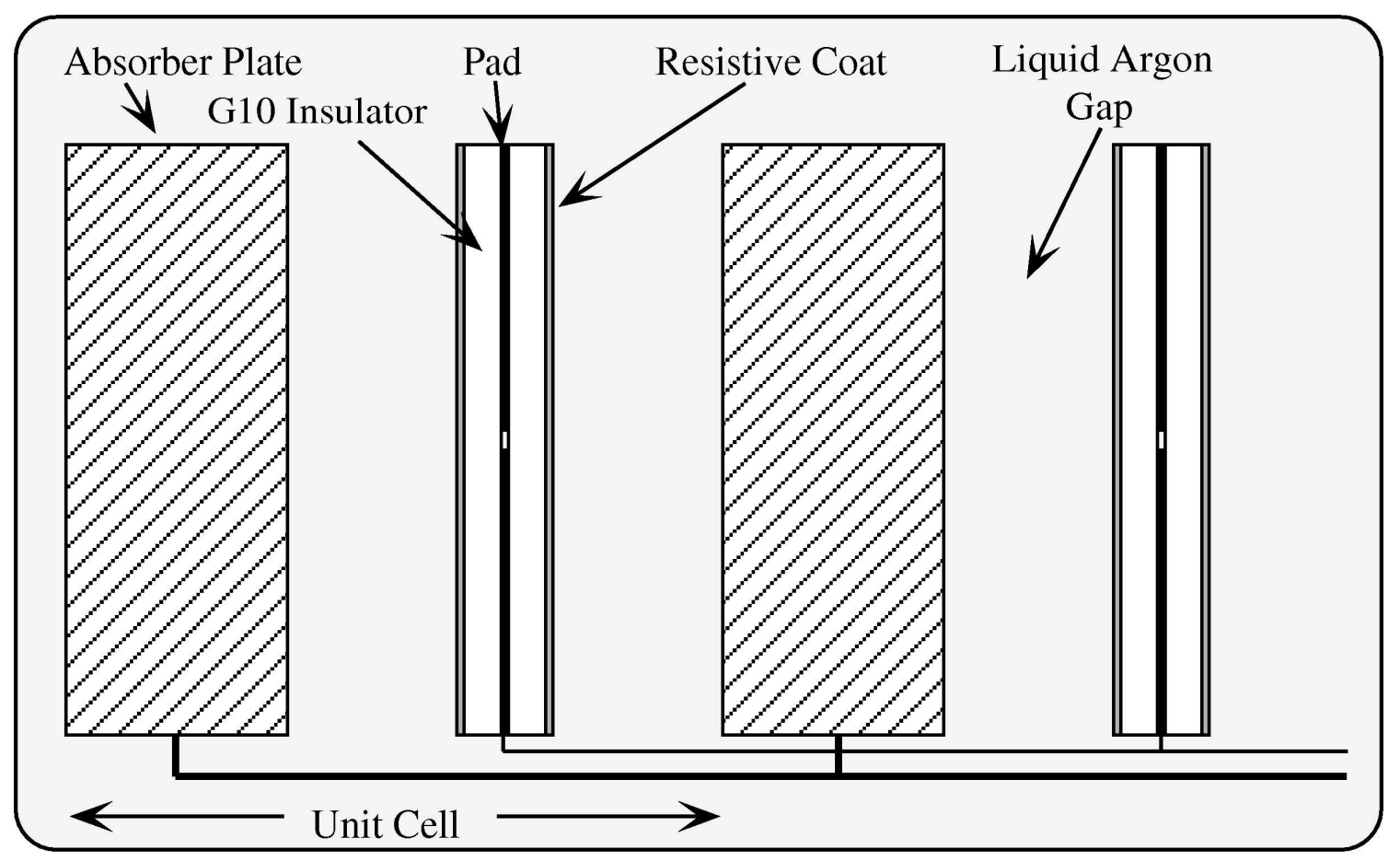

Figure 2.17: A D $\varnothing$ calorimeter cell with alternating layers of absorber plates, liquid argon, and signal boards. Many cells form the layers of the calorimeter. Absorber plates can vary in size, but the gap between the absorber plate and the signal board is always $2.3 \mathrm{~mm}$.

\subsubsection{Muon System}

The muon system (Figure 2.18) is the outermost detector system in the D $\varnothing$ detector $[24,25]$. It is separated into the central muon detectors and the forward muon detectors. Muons produced in collisions at $\mathrm{D} \varnothing$ are too massive to be stopped in the calorimeter, and move too fast to decay before leaving the detector. They can be detected by the tracking system and calorimeter, but the muon system provides the ultimate confirmation. This is accomplished using the various detectors in the muon system and the iron toroid magnet, which allows a crude measurement of the muon momentum independent of the tracking system.

The wide angle muon system (WAMUS) covers the region $|\eta|<1$ and has three layers (called A, B, and C). The A layer lies between the calorimeter and the toroid magnet, and the B and $\mathrm{C}$ layers are outside of the magnet. The WAMUS is composed 


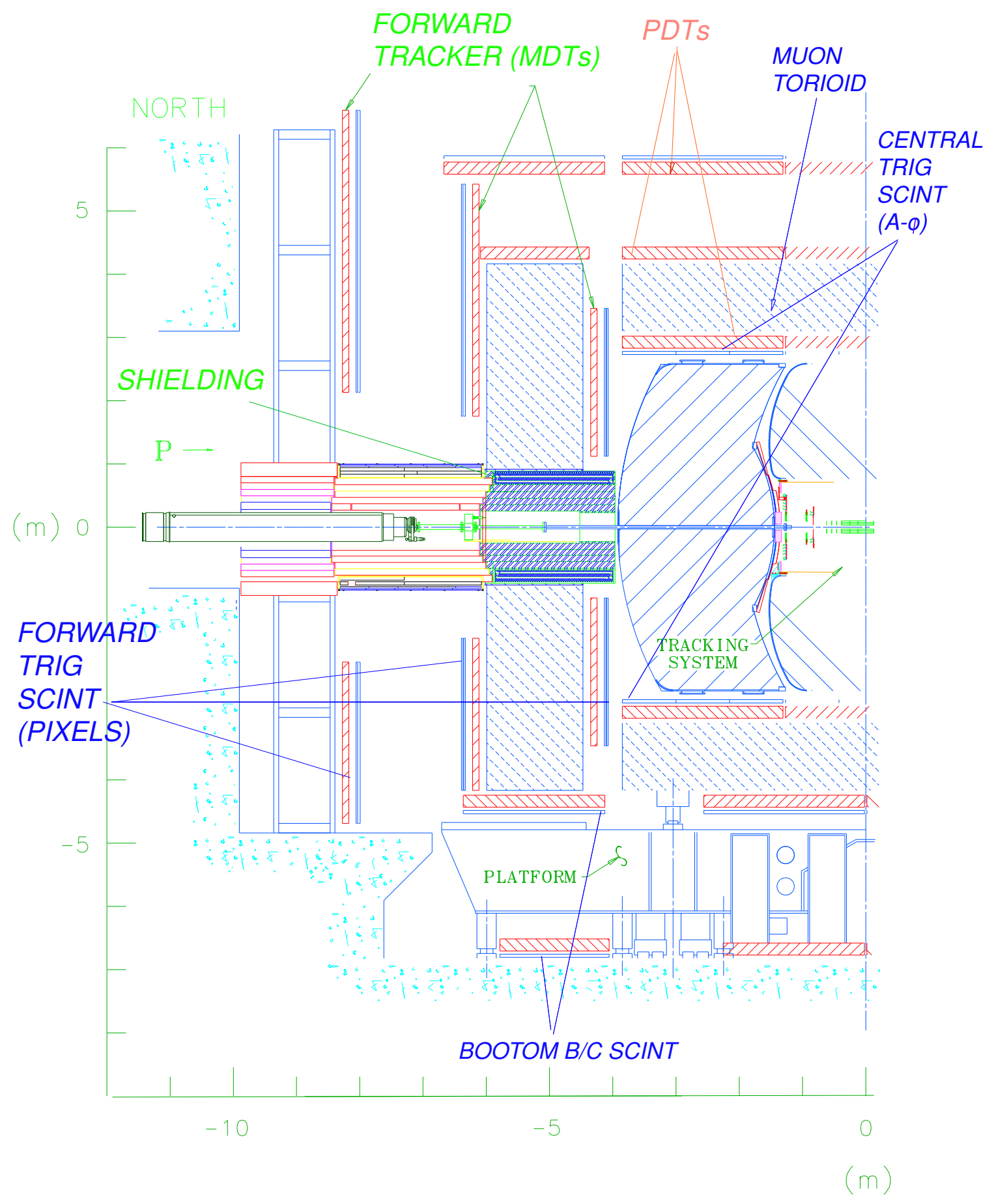

Figure 2.18: The D $\varnothing$ muon system, including scintillator (scint) and proportional and mini-drift tube chamber layers (PDT and MDT).

of 94 proportional drift tube chambers (PDTs) in three layers (A, B, and C). There are scintillators between the A layer and the calorimeter, and an outermost layer of 


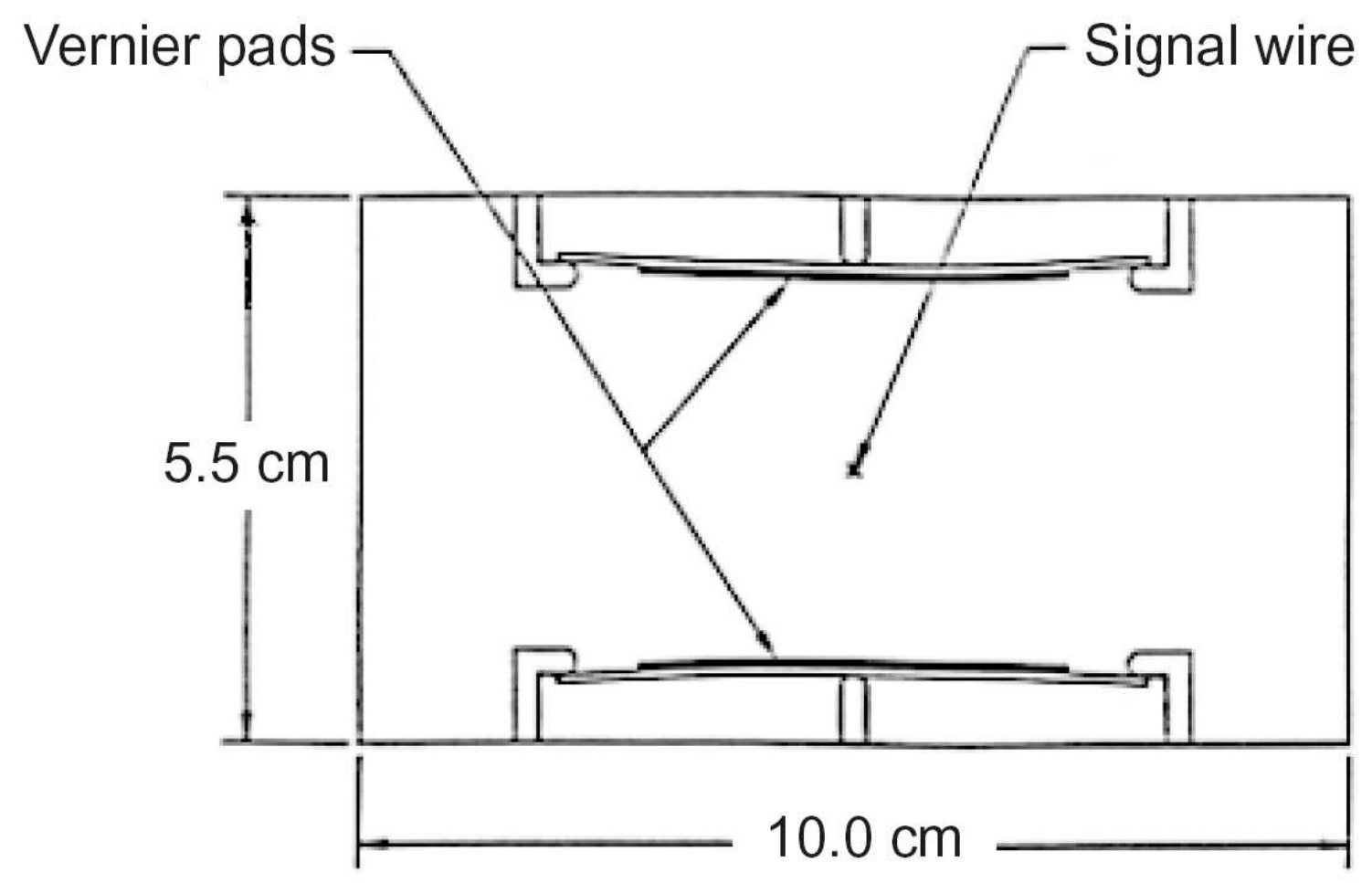

Figure 2.19: A muon proportional drift tube unit cell consisting of an anode sense signal wire in a gas-filled chamber and two sets of cathode pads.

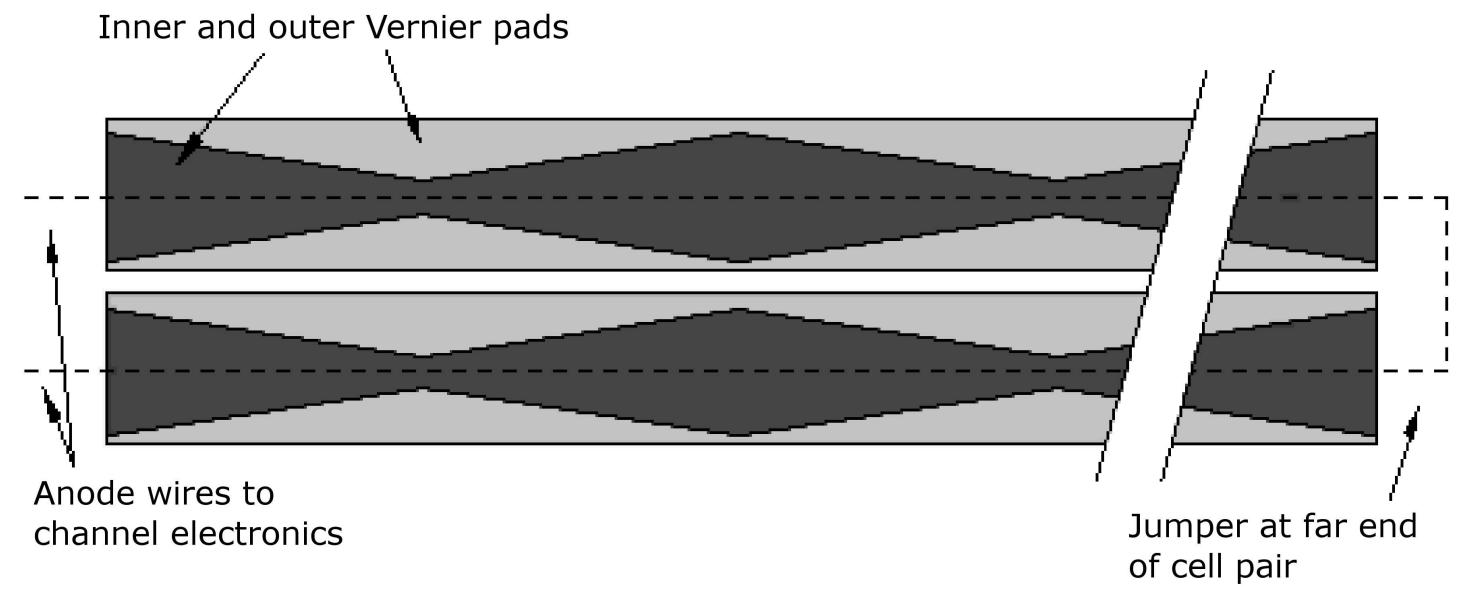

Figure 2.20: A proportional drift tube unit cell viewed from above.

scintillators is used to reject cosmic rays.

The PDTs have three or four decks of drift cells. The PDTs are rectangular in shape, approximately $10.1 \mathrm{~cm}$ wide and $5.5 \mathrm{~cm}$ high, with one anode sense wire and two sets (inner and outer) of cathode pads per drift cell (see Figures 2.19 and 2.20). 


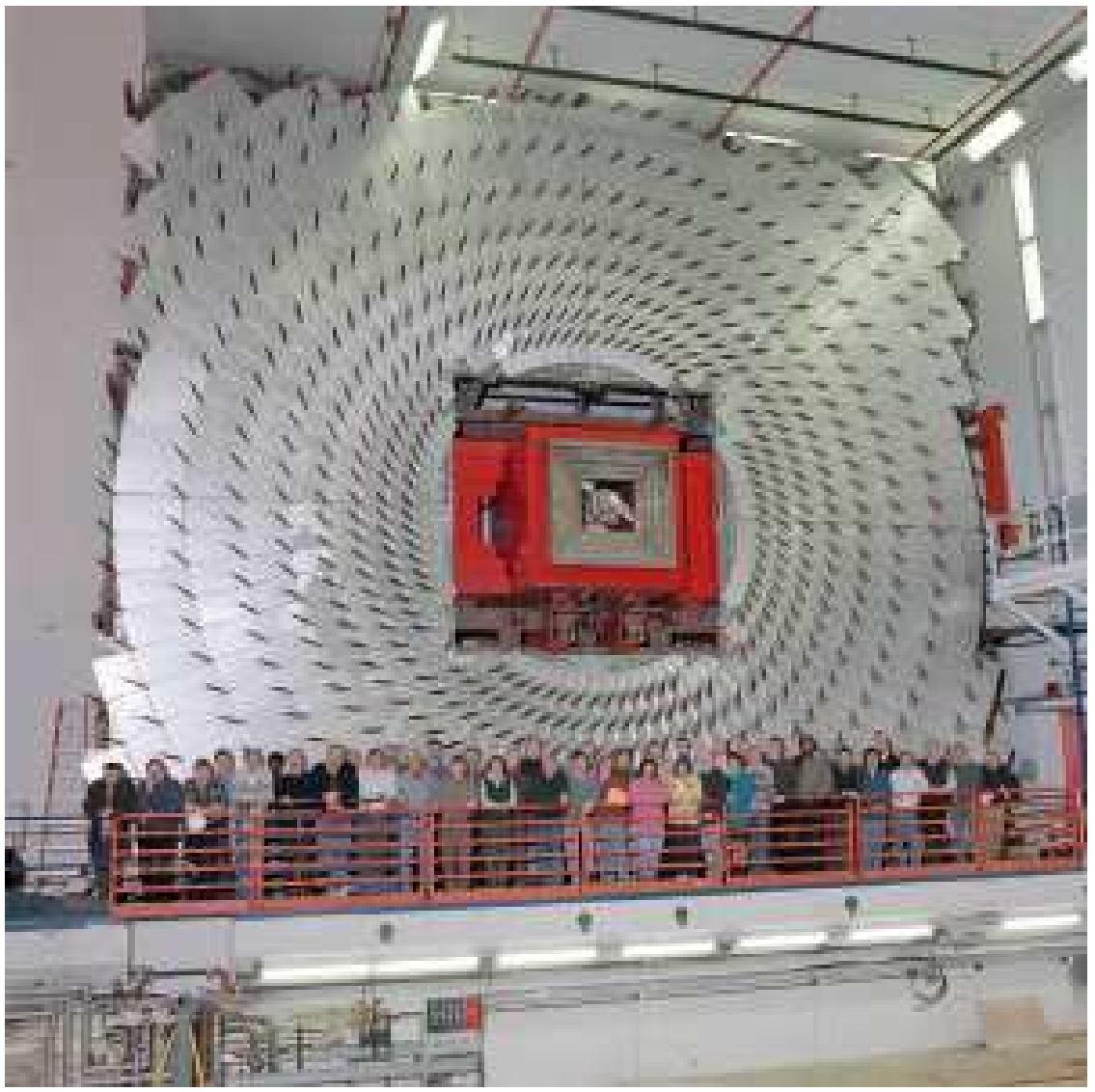

Figure 2.21: Part of the C layer of the D $\varnothing$ forward muon system.

Adjacent unit cells share anode wires, allowing a time difference measurement between the arrival of signals from the two wires. The cells are filled with $80 \%$ argon, $10 \%$ methane, and $10 \% \mathrm{CF}_{4}$. The voltage is approximately $2.5 \mathrm{kV}$ for the pads and $5.0 \mathrm{kV}$ for the wires. Muons that traverse the gaseous region ionize electrons that accumulate on the wires and induce a charge on the pads. The ratio of the charges on the pads, the drift time, and the time difference between the arrival of charge from adjacent anode wires allow the determination of the muon's position. Subsequent measurements allow a trajectory calculation, and the bend in the muon's path due to the toroid magnet allows a charge and momentum measurement.

The forward angle muon system (FAMUS, see Figure 2.21) covers the region $1<|\eta|<2$. It consists of three layers (A, B, and C) of mini-drift tubes (MDTs), 
three layers of scintillation counters, and the toroidal magnet. There are three or four planes of tubes in each of the MDT layers, and every tube has eight $1 \times 1 \mathrm{~cm}^{2}$ cells with a $50 \mu \mathrm{m}$ anode wire in the center. MDTs have excellent coordinate resolution, short electron drift time, and high segmentation.

The scintillation counters are mounted on the inside (layers A and C) or outside (layer B) of the FAMUS MDT planes. The counters are made of scintillator plates cut in a trapezoidal shape. Wavelength shifting bars are attached to the sides of the plates for light collection. The bars transfer light to the phototubes with a quantum efficiency of approximately 15\%. The scintillators are used for triggering and track reconstruction.

\subsection{The DØ Trigger and Data Acquisition Systems}

Proton-antiproton collisions occur inside the $\mathrm{D} \varnothing$ detector at a maximum rate of approximately 2.5 MHz. Most of the collisions are not interesting in the context of the physics program at $\mathrm{D} \varnothing$. This fact, along with the realities of finite storage resources and limits on the data-transfer rate to the storage facility, lead to the decision to write events to permanent storage at a rate of approximately $50 \mathrm{~Hz}$. DØ employs a three-level trigger system to quickly characterize all of the events and select the events to be written to permanent storage $[15,18]$.

The Level 1 (L1) trigger, mainly composed of custom-built electronics, reduces the event rate to approximately $5 \mathrm{kHz}$ using coarse information from the detector. Coarse, or lower resolution, data must be used to allow L1 to keep up with the rate of collisions. The Level 2 (L2) trigger is designed to be able to process data at a rate of up to $10 \mathrm{kHz}$, although it is unlikely to ever operate at this rate. It uses a combination of custom-built electronics and commodity processors to make trigger decisions based on more detector information and with more time than the L1 trigger. 
The L2 trigger reduces the data rate to approximately $1 \mathrm{kHz}$ for the L3 trigger. The L3 trigger is the only trigger level that has the full detector readout available, and selects approximately 50 events per second to be written to tape.

\subsubsection{The Level I Trigger}

The L1 trigger is responsible for reducing the data rate from $2.5 \mathrm{MHz}$ to approximately $5 \mathrm{kHz}$. It uses custom-built electronics, and relies on information from the central fiber tracker, the calorimeter, the preshower system, and the muon system.

Up to 128 different L1 trigger conditions can be required when programming the L1 trigger. An event that satisfies any of the requirements will cause the event to pass the trigger. This allows a diverse set of physics conditions to trigger the event's passage. The first requirement in most L1 triggers is the presence of an inelastic collision, which is detected by small-angle counters.

\section{L1 Calorimeter Trigger}

The L1 calorimeter (L1CAL) trigger is based on information from the D $\varnothing$ calorimeter system (see Section 2.2.6).

The L1CAL trigger towers are formed by grouping together calorimeter cells in $\Delta \eta \times \Delta \phi=0.2 \times 0.2$ regions. The energies of all the cells in a tower are summed to calculate the energy of a trigger tower. The use of 1280 trigger towers for triggering is an example of the way the L1 trigger makes use of coarse detector information by reducing the resolution of the detector data.

The transverse energy in the trigger towers is measured by first summing up all of the energy in the cells contained in each trigger tower. The energy of each tower is then multiplied by the sine of the trigger tower polar angle (there is an implicit assumption that the event vertex is at $z=0$ ). The transverse energy contained in the electromagnetic (EM) layers is summed separately from the total (TOT) trans- 
verse energy for each trigger tower. The total transverse energy sum excludes energy contained in the coarse hadronic layers of the calorimeter.

L1 trigger conditions are specified by a certain number of trigger towers and a minimum transverse energy threshold for the towers. At any one time, there are a maximum of four different thresholds for the EM and TOT towers separately. An example L1 trigger condition is two TOT trigger towers, each registering transverse energy greater than $5 \mathrm{GeV}$.

The L1CAL trigger system also calculates the sum of all of the transverse energy in the calorimeter. L1CAL trigger conditions can be based on the total transverse energy or the imbalance of transverse energy (the missing transverse energy, MET). Total energy and MET triggers are each specified by a minimum threshold.

The L1CAL trigger passes the trigger tower energies to the L2 trigger to be used in L2 trigger decisions.

\section{L1 Central Track and Preshower Triggers}

The L1 central track trigger (L1CTT) uses scintillator information from the CFT (Section 2.2.4) and the FPS and CPS detectors (Section 2.2.5). The L1CTT makes trigger decisions and provides the L2 and L3 triggers with sorted lists of tracks and preshower clusters. In the central detector region, the CFT and only the axial strips from the CPS detector are used. In the forward region, the FPS detector is used in conjunction with the CFT.

Every bunch crossing (every 132 ns), discriminator bits are read out from the tracking and preshower detectors. These outputs are fed into a series of field-programmable gate arrays (FPGAs) which use preloaded logic to compare the bits to various hit patterns. The hit patterns correspond to possible tracks, and a match to a hit pattern corresponds to the finding of a track with a momentum in one of four $\mathrm{p}_{\mathrm{T}}$ bins: $1.5-$ $3 \mathrm{GeV} / \mathrm{c} ; 3-5 \mathrm{GeV} / \mathrm{c} ; 5-10 \mathrm{GeV} / \mathrm{c}$; or above $10 \mathrm{GeV} / \mathrm{c}$. The trigger is divided into 


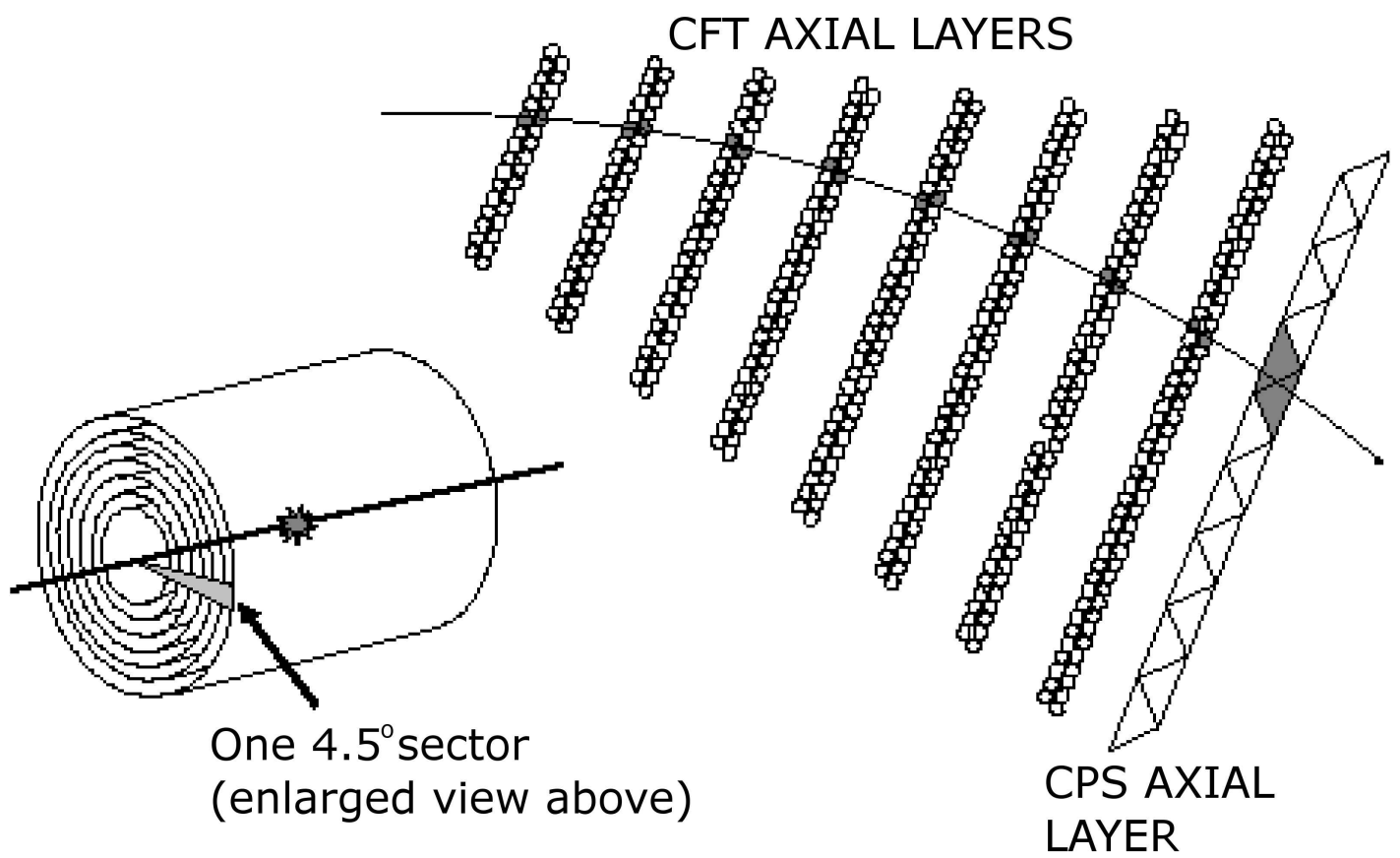

Figure 2.22: A single 4.5 degree central fiber tracker (CFT) sector along with a hypothetical track and hits in all eight CFT layers and the central preshower (CPS) axial layer.

4.5 degree sectors, and a maximum of six tracks can be found per event per sector. Figure 2.22 shows the track of a hypothetical particle which deposits energy in all eight CFT layers and the CPS axial layer. Trigger conditions can be specified by a certain number of tracks above a $\mathrm{p}_{\mathrm{T}}$ threshold, with or without the requirement of a CPS cluster match to the track.

\section{L1 Muon Trigger}

The L1 muon trigger relies on information from the muon wire chambers and scintillation counters (Section 2.2.7), and tracks from the L1CTT. FPGAs in four crates on the detector platform are used to combine information from these detectors. The muon triggers are first separated into central and forward regions, and further divided into octants. Triggers in each region are built up from octant decisions.

Triggers can require muons in the central $(|\eta|<1)$, forward $(1<|\eta|<2)$, wide 
$(|\eta|<1.2$ to match the central tracking coverage), or all regions $(|\eta|<2)$. Matches to tracks can also be required. If a match to a track is required, the four $\mathrm{p}_{\mathrm{T}}$ bins from the tracker are used as trigger thresholds.

\subsubsection{The Level II Trigger}

The L2 trigger was designed to process data at a rate between $5-10 \mathrm{kHz}$ and reduce it to approximately $1 \mathrm{kHz}$. In practice, front-end crate readout problems have limited the input rate to $\mathrm{L} 2$ to less than $2 \mathrm{kHz}$.

The L2 trigger system is divided into six crates. There are five preprocessor crates that process data from both the L1 trigger system and directly from the detector through the front-end crates. There is also a Global crate that collects data from the five preprocessor crates and makes trigger decisions by correlating the data and imposing trigger requirements.

Each of the six L2 crates contains custom-built electronics. The crates work to process the data and return a trigger decision. This must happen within approximately $100 \mu$ s at a rate of $10 \mathrm{kHz}$, but more time is currently available due to current L1 accept rates being lower than $10 \mathrm{kHz}$.

Beta processors [26], running identical executables but configured differently, are installed in each crate. A processor's configuration specifies which algorithm runs in the processor's executable and corresponds to the type of data being processed in the crate.

The data processed by L2 is not raw detector data, but is coarse or abbreviated data that can be processed quickly enough to allow the reduction of the rate to L3, where the full detector readout is available. L2 exists because the full detector cannot be read out at a rate much greater than $1 \mathrm{kHz}$. 


\section{L2 Calorimeter Preprocessor}

The L2 calorimeter preprocessor [27] crate receives data from the L1CAL system. L2 receives the trigger towers created at L1 and combines them to form electromagnetic objects (for electron and photon triggering) and jets. Some of the trigger towers from L1 are marked as seed towers. They have transverse energy above some threshold and are used as the starting point in forming L2 electromagnetic objects and jets.

Electromagnetic objects are formed by adding the energies of two trigger towers. An electromagnetic object is formed from each seed and its highest-energy neighbor. The algorithm can be configured not to add the neighbor's energy under specified conditions. Quantities called isolation and EM fraction measuring the objects' isolation from other energy in the calorimeter and the fraction of energy in the calorimeter's electromagnetic layers are calculated for use in the Global processor.

Jets are formed by summing the energy in a five-by-five trigger tower array with the seed tower at the center. Two seeds that are very close together will not both be used to form jets. Only the higher seed will be used.

After the electromagnetic objects and jets are properly formatted they are sent to the Global processor.

\section{L2 Muon Preprocessors}

There are two preprocessor crates for the L2 muon system. The L2 central muon preprocessor [28] and the L2 forward muon preprocessor [28] crates process information from the central and forward muon detectors (see Section 2.2.7).

Inputs to the L2 muon system are first received by second level input computers (SLICs), where most of the processing is done. One of several different algorithms, which differ due to the geometry of the detector, processes the data and forms muon stubs. These stubs are created for the A, B, and C layers of the muon system and are the result of the combination of scintillator and drift tube information in each of 
these layers. The stubs are passed to the Beta processor, which forms muon objects out of the stubs and sends them to the Global processor. The muon objects include position, momentum, sign, and timing information.

The muons are assigned a quality by the global processor: tight; medium; or loose. The definitions are as follows:

- tight

- at least two A layer hits and two BC layer hits in the central region

- sum of A layer hits and BC layer hits greater than 3 in the forward region

- medium

- at least one A layer hit and one BC layer hit

\section{- loose}

- at least one A layer hit or one BC layer hit in the central region

- sum of A layer hits and BC layer hits greater than 1 in the forward region.

\section{L2 Tracking Preprocessor}

The L2 tracking preprocessor crate, currently being commissioned, uses tracks from the L1CTT and from the silicon detector. L2 tracks based solely on the L1CTT tracks are called L2CTT tracks. The tracks based on the L1CTT tracks and extrapolated into the silicon detector are called L2STT tracks. L2CTT and L2STT tracks share an identical data format, so they can be used interchangeably by the Global processor.

Information associated with tracks includes position, momentum, and sign. Other quantities which are measured for L2STT tracks are: the quality of the track using

a $\chi^{2}$ parameter; the track fit quality; the impact parameter; and impact parameter significance. Track objects are filled with this information and sent to the Global processor, sorted by $\mathrm{p}_{\mathrm{T}}$ or impact parameter significance. 


\section{L2 Preshower Preprocessor}

The L2 preshower preprocessor crate (L2PS) is responsible for the formation of clusters for the CPS and FPS detectors (see Section 2.2.5). The information received from the L1 trigger includes one-dimensional clusters of energy deposition. L2PS forms two-dimensional clusters by checking for overlaps of the one dimensional clusters. The two-dimensional clusters are sent to the Global processor for use in triggering.

\section{L2 Global Preprocessor}

The L2 Global worker makes trigger decisions based on the objects created by the L2 preprocessors. L2 trigger decisions are made by first creating Global physics objects. These objects can be based directly on the objects reported by the preprocessors or can be created by combining objects from different preprocessors. The L2 Global worker imposes cuts on the Global physics objects according to configuration information it receives from the trigger control computer based on the trigger list. A complete description of the L2 Global worker is included in Appendix A.

\subsubsection{The Level III Trigger and Data Acquisition}

The L3 trigger is responsible for reducing the data rate from approximately $1 \mathrm{kHz}$ to $50 \mathrm{~Hz}$. It uses a Cisco 6509 Ethernet switch, standard PCs, and commodity VME single-board computers [29].

The L3 data acquisition system receives digitized data from all of the detector subsystems. It then routes the data to filter processes running on PCs in the L3 trigger farm. The processes are configured through a supervisor process running on a dedicated CPU which is the interface between the L3 trigger system and the run control system, COOR.

The L3 trigger has full access to the D $\varnothing$ data, and so can offer a complete menu 
of triggering options. It is not restricted to a one-to-one correspondence with L1 and L2 triggers, and so several slightly different conditions can be applied to optimize the efficiency and rejection of an L3 trigger condition. The L3 trigger is as similar as possible to the offline reconstruction code, the only restriction being the amount of time allowed for processing each event. The L3 trigger makes available electromagnetic, muon, tau, track, and several other triggers.

After an event passes the L3 trigger, it is transferred over a separate network connection to the Feynman Computing Center for permanent storage. 


\section{Chapter 3}

\section{Offline Event Reconstruction}

The $\mathrm{D} \varnothing$ detector (described in Chapter 2) is designed to detect and identify the particle remnants of proton-antiproton collisions. Particles produced in collisions interact with the detector, and records of these interactions are stored as raw data. To study the physics of particle interactions, it is necessary to reconstruct, from the raw data, the kinematic properties of the particles that emerged from the original interaction. This reconstruction is done through the use of a software program called "dØreco" [30], written in the C++ programming language.

The dØreco program is separated into many software packages, collections of files containing $\mathrm{C}++$ source code, each responsible for one piece of the total event reconstruction. There is one dØreco executable program run on several computer farms, each containing hundreds of PCs. Each farm is assigned a subset of the total data

recorded by $\mathrm{D} \emptyset$. New versions of $\mathrm{d} \emptyset$ reco, organized using version numbers, are created periodically as updates and improvements become available. The data used in this analysis were reconstructed using p14.x versions of dØreco. The dØreco program is the result of many years of work by many people, and in this section only an overview of the basic concepts of particle reconstruction is presented. 


\subsection{Track and Vertex Reconstruction}

Charged particles passing through the DØ SMT and CFT detectors leave energy in many layers of the detectors through which they pass (see Sections 2.2.3 and 2.2.4). These localized depositions of energy in each layer of the tracking detectors are called hits. In addition to the hits from high-momentum charged particles traversing the detector (which are of primary interest in this analysis), there are also hits from lowmomentum tracks from jets, tracks from secondary collisions in the event, tracks from particles interacting with the beam pipe, and random electronic noise. For any event, there are many such hits in the tracking detectors which make the reconstruction of tracks difficult.

\subsubsection{Track Algorithm}

Several different algorithms have been used to reconstruct tracks in the $\mathrm{D} \varnothing$ detector. Algorithms are chosen for each analysis based on the unique requirements for each analysis. The most common algorithm, used in this analysis, is now a combination of the AA track algorithm [31] and the Hough transform (HTF) algorithm [32]. Each runs separately to generate a list of track candidates. Then the two lists are combined, and duplicates are removed, before final fitting is done.

The AA track algorithm starts by generating a pool of track candidates using the hits in the SMT. The algorithm selects all sets of three hits which lie along a path originating from the beam spot. It then extrapolates the path of the track outward to either the next layer of the SMT or to the CFT, to calculate the location where the track would have crossed the next layer. The algorithm checks whether there is a hit near this location, and then extrapolates to the next layer, and repeats the procedure. At each layer, it calculates a $\chi^{2}$ value for the track and associates the hit with the track if the $\chi^{2}$ value is below a configurable value. New tracks can be constructed if 
there are multiple hits in a layer that produce acceptable tracks. If there is no hit in a given layer, the algorithm continues and tallies a miss for that track. At the end of this procedure, a list of track candidates is produced along with a number of hits, misses, and $\chi^{2}$ for each track.

A list of vertices is next constructed by following the tracks back to the $z$-axis. This list of vertices is used to look for more track candidates that only have hits in the CFT. The same extrapolation procedure is repeated as above, starting in the CFT, but with the constraint that the track start at a primary vertex. CFT-only tracks have been used in several analyses, and their use can give an increase in efficiency. Each analysis must decide whether the corresponding increase in background is acceptable.

The trajectory of a particle in the plane perpendicular to a magnetic field is a circle characterized by three parameters: its curvature; impact parameter; and direction at the point of closest approach. The HTF algorithm begins by dividing this parameter space into bins. Each pair of hits in the tracking detector corresponds to a point in the parameter space. For each point, the algorithm increments a histogram bin corresponding to the point in the parameter space. All hits from a track correspond to the same bin, so a peak corresponds to a track. The HTF algorithm uses this technique to generate a list of tracks.

The separate lists of tracks from the AA Track algorithm and the HTF algorithm are combined into one list. Once the complete list of tracks has been generated, it is sorted by number of hits, fewest misses, and lowest $\chi^{2}$, in that order. The best track is automatically kept, and the rest of the tracks are examined. The number of hits that each of the remaining tracks shares with the already-selected tracks is calculated. If the number of shared hits is less than $2 / 3$ the number of total hits, or the number of unique hits is greater than three, the track is kept. 


\subsubsection{Selecting the Primary Vertex}

After a list of tracks and a list of vertices have been generated, the primary vertex must be selected [33]. The primary vertex is used by dØreco in the reconstruction of jets, b-jets, electrons, and missing transverse energy. Other vertices in the event may be due to minimum-bias interactions. Minimum-bias interactions are protonantiproton interactions besides the primary hard scatter, which generally produce particles with low $\mathrm{p}_{\mathrm{T}}$.

The first step in selecting the primary vertex is to group all of the vertices in clusters. Vertices within $2 \mathrm{~cm}$ of each other in the $z$ direction are grouped together. For every cluster, the highest multiplicity vertex is chosen and added to a new list of selected vertices. For each of the selected vertices, the probability that each track comes from a minimum-bias vertex is calculated relative to a $\mathrm{p}_{\mathrm{T}}$-dependent function derived from a minimum-bias Monte Carlo sample. Higher $\mathrm{p}_{\mathrm{T}}$ tracks are less likely to come from minimum-bias vertices, and have a lower minimum-bias probability. The minimum-bias probabilities are combined to calculate a minimum-bias vertex probability. The vertex with the smallest minimum-bias vertex probability is chosen as the primary vertex.

\subsubsection{Bottom-Quark Jet Identification}

Identification of b-quark jets is extremely important for the $\mathrm{D} \emptyset$ physics program. Bottom-quark jets are jets of particles that originate from $b$ quarks, and are important because $\mathrm{b}$ quarks appear in the decay chains of many particles of interest.

Bottom quarks are identified, or tagged, using several different b-tagging algorithms. These include:

\section{- Secondary vertex tagging algorithm}

Reconstructs the decay vertex of long-lived B hadrons within jets. The decay 
of a long-lived hadron produces several charged particles emanating from a secondary vertex, displaced from the primary interaction point [34].

\section{- Jet lifetime probability algorithm}

The impact parameters of all tracks associated with a calorimeter jet can be combined into a single variable, the jet lifetime probability, which is used to tag b jets [35].

\section{- Counting signed impact parameter algorithm}

Relies on the fact that tracks produced by charged decay products of long-lived $\mathrm{B}$ hadrons have a non-zero impact parameter with respect to the primary vertex. The sign of the impact parameter is given by the sign of the impact parameter projection on the jet axis, determined based on calorimeter information. Impact parameter significance is defined as the impact parameter divided by its uncertainty calculated by the track finding algorithm [36].

\section{- Soft lepton tagging algorithm}

Relies on the fact that $\mathrm{B}$ hadrons are more likely to decay to a final state containing a muon than lighter hadrons. This algorithm tags a jet as a b jet if there is a muon around the jet within a cone of $\Delta \mathrm{R}=\sqrt{(\Delta \eta)^{2}+(\Delta \phi)^{2}}<0.5[37]$.

\subsection{Muon Reconstruction}

The p14.x versions of dØreco rely on muons detected using the forward and central muon detectors (see Section 2.2.7), and also makes use of the tracking detectors and the calorimeter (see Section 2.2.6). Muons reconstructed using only the information from the muon detectors are called "local" muons, and those that also have a matching track are called "central track-matched" muons. Muons can also be reconstructed using minimum-ionizing particle (MIP) signatures in the calorimeter. The 
Muon Tracking in the Calorimeter (MTC) algorithm identifies MTC muons using MIP signatures, although these are not used in this analysis.

\subsubsection{Muon Types and Qualities}

The dØreco program classifies muons by type and quality. The types of muons are differentiated by a parameter nseg. The absolute value of nseg is the number of segments associated with the reconstructed muon object (this can be between 0 and

3, with a potential segment in each of the A, B, and C layers of the muon system), and the sign of nseg is determined by whether or not there is a central track matched to the muon. Muons with $|n s e g=1|$ have hits only in the A layer of the muon system, $\mid$ nseg $=2 \mid$ muons have hits in the B and/or C layers of the muon system, and $|n s e g=3|$ muons have hits in the A layer and in the $\mathrm{B}$ and/or $\mathrm{C}$ layers of the muon system.

The quality of a reconstructed muon is determined by how many hits it has associated with it in each layer of the muon system. The possible values of quality are loose, medium, and tight, with loose having the highest efficiency and highest background, and tight having the lowest efficiency and lowest background. The number of hits required for each value of quality depends on the detector geometry (the bottom of the detector has less instrumentation due to the presence of detector supports), but in general more hits are required for a muon to have tighter quality. The requirements for the various types of muons are [38]:

\section{- Tight muons}

Only $|n s e g|=3$ muons can be tight. A muon is tight if it has

- at least two A layer wire hits

- at least one A layer scintillator hit

- at least three BC layer wire hits 
- at least one BC scintillator hit

- and a converged local fit $\left(\chi_{\text {loc }}^{2}>0\right)$.

- $\mid$ nseg $\mid=3$ medium/loose muons

When an $\mid$ nseg $\mid=3$ muon candidate fails the tight criteria it can still be medium or loose. An $|n s e g|=3$ muon is medium if it has

- at least two A layer wire hits

- at least one layer scintillator hit

- at least two BC layer wire hits

- and at least one BC scintillator hit (except for central muons with less than four BC wire hits).

An $|n s e g|=3$ loose muon has the same definition as a medium muon, but one of the above tests is allowed to fail, with the A wire and scintillator requirement treated as one test, and at least one scintillator is always required.

- $n s e g=2$ loose/medium muons

Muons with $|n s e g|<3$ can only be loose or medium if they are matched to a central track, and so have $n s e g>0 . n s e g=2$ muons are muons with a $\mathrm{BC}$ segment matched with a central track. An $n s e g=2$ muon is loose if it has

- at least one BC scintillator hit

- at least two BC layer wire hits.

An $n s e g=2$ muon is defined as medium if it fulfills the above requirements and if it is located in the bottom part of the detector (octant 5 and 6 with $\mid$ detector $\eta \mid<1.6)$. 
- $n s e g=1$ loose/medium muons

Muons with $n s e g=1$ are muons with an A segment matched with a central track. An $n s e g=1$ muon is loose if it has

- at least one scintillator hit

- at least two A layer wire hits.

An $n s e g=1$ muon is defined as medium if it fulfills the above requirements and if it is located in the bottom part of the detector (octant 5 and 6 with $\mid$ detector $\eta \mid<1.6)$.

Each analysis can choose which type and quality of muon to use based on the needs of the analysis. Efficiency and background measurements are generally done separately in each analysis.

\subsubsection{Muon Isolation}

Muons not produced in association with jets are called isolated muons. To be considered isolated, a muon must satisfy the following requirements (suggested in [39]):

- The energy measured in the calorimeter in the region defined by a 0.1-radius cone and 0.4-radius cone in $\eta-\phi$ space centered around the muon (the hollow cone energy) must be less than $2.5 \mathrm{GeV}$ (see Figure 3.1). The energy that the muon deposits in the calorimeter should be along the path the muon traveled in the calorimeter, while significant energy further away from the path is likely to come from an associated jet.

- The scalar sum of the transverse momenta of all tracks (except the one matched to the muon) contained within a cone of radius 0.5 around the muon must be less than $2.5 \mathrm{GeV} / \mathrm{c}$. Extra tracks around the muon are indicative of the presence of a jet, which contains many charged particles. 


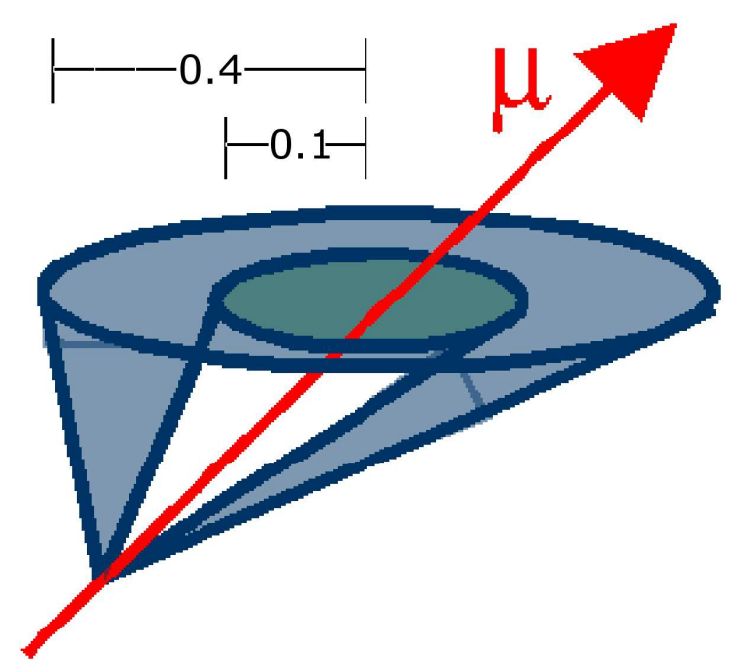

Figure 3.1: The muon isolation variable in the calorimeter. The energy contained in a cone of radius 0.1 around a muon is subtracted from the energy contained in a cone of radius 0.4 .

\subsection{Jet Reconstruction}

Partons (quarks and gluons) are detected at D $\varnothing$ by the measurement of their hadronization products, jets of hadrons which are created due to the color confinement of the quarks. The dØreco code measures the energies of jets [40]. For use in analysis, an extrapolation back to the energies of the original partons, which are usually the object of interest to the analyzer, must be done.

\subsubsection{Jet Cone Algorithm}

Jet reconstruction starts with the clustering of calorimeter energies into towers with size $\Delta \eta \times \Delta \phi=0.1 \times 0.1$. Towers with $\mathrm{E}_{\mathrm{T}} \geq 0.5 \mathrm{GeV}$ are called seeds, and become the basis for the formation of jets. Next, preclusters are formed by adding all of the energy within a cone of $\mathrm{R}=0.3$ around each seed, where $\mathrm{R}$ is defined as

$$
R=\sqrt{(\Delta \eta)^{2}+(\Delta \phi)^{2}}
$$


Preclusters with $\mathrm{E}_{\mathrm{T}} \geq 1.0 \mathrm{GeV}$ are kept.

Using all the preclusters within $\mathrm{R}_{\text {final }}$, where $\mathrm{R}_{\text {final }}$ is the final size of the jet cone used (different values of $\mathrm{R}_{\text {final }}$ are used in different analyses), jet $\eta$ and $\phi$ directions and energies are estimated using

$$
\begin{gathered}
\eta=\frac{\sum_{i} E_{T}^{i} \eta^{i}}{\sum_{i} E_{T}^{i}} \\
\phi=\frac{\sum_{i} E_{T}^{i} \phi^{i}}{\sum_{i} E_{T}^{i}} \\
E_{T}=\sum_{i} E_{T}^{i}=\sum_{i} E_{i} \sin \left(\theta_{i}\right),
\end{gathered}
$$

where $i$ is the $i$-th energy deposited in the jet.

All energy deposits within $R_{\text {final }}$ around the axis of a jet are summed and a new direction and energy are calculated. This is repeated until the direction is stable.

If two stable jets are separated by a distance of at least $R$ and at most $2 R$, a new jet axis is formed at the midpoint between the two jets and used as a precluster. If two jets share energy, they are merged if the amount of shared energy is more than half of the energy of the lower-energy jet. Jets with $\mathrm{E}_{\mathrm{T}}<8.0 \mathrm{GeV}$ are discarded.

\subsubsection{Jet Energy Scale}

To determine the parton energy from the jet energy, several effects must be understood [40]. The term "offset energy" describes energy measured in a jet that does not come from the original parton, and leads to an offset that must be corrected for in the energy measurement. Offset energy comes from uranium noise, interactions between the other partons in the proton and antiproton (known as the underlying event), previous interactions, and multiple hadron collisions in the event. This may also depend

on the instantaneous luminosity. The calorimeter response, or the relationship between the energy measured in the calorimeter and the energy of the incident particle, 
must also be known. Finally, the amount of energy contained inside the jet cone and how much energy enters the jet cone from showers of other particles must be known.

The energy of a parton is determined from a jet using the equation

$$
\mathrm{E}_{\text {parton }}=\frac{\mathrm{E}_{\text {jet }}-\mathrm{E}_{\text {offset }}}{\mathrm{S}_{\text {jet }} \cdot \mathrm{F}_{\mathrm{s}}}
$$

where $\mathrm{E}_{\text {parton }}$ is the energy of the parton, $\mathrm{E}_{\text {jet }}$ is the measured energy of the jet, $\mathrm{E}_{\mathrm{offset}}$ is the offset energy from the sources mentioned above, $\mathrm{S}_{\text {jet }}$ is the response of the calorimeter to jets, and $F_{\mathrm{s}}$ is the fraction of the jet contained within the cone.

These quantities are studied by the jet energy scale group at $D \varnothing$ and derived in various ways. For example, uranium noise is determined and underlying event studies are conducted using data taken in special runs. $\mathrm{S}_{\text {jet }}$ can be measured as a function of jet $\mathrm{E}_{\mathrm{T}}$ with $\gamma+$ jet events, using the energy of the photon as a reference since photons can be measured more precisely than jets and calibrated more easily. Out-ofcone showering can be measured using jets in the data, by comparing the energy in the cone to the energy in a larger cone around the jet axis. The average out-of-cone energy is estimated as a function of jet $\mathrm{E}_{\mathrm{T}}$.

\subsection{Electromagnetic Object Reconstruction}

Electromagnetic objects are reconstructed using several different algorithms, with each analysis choosing the one most suitable for its physics. Here, the simple cone algorithm is described [41].

First, clusters of adjacent cells with transverse energy greater than $1.5 \mathrm{GeV}$ and a ratio of energy in the electromagnetic layers to total energy (EM fraction) above 0.9 are identified. Next, the isolation of the cluster is calculated, defined as

$$
\text { isolation }=\frac{\text { EisoTot }- \text { EisoCore }}{\text { EisoCore }} .
$$




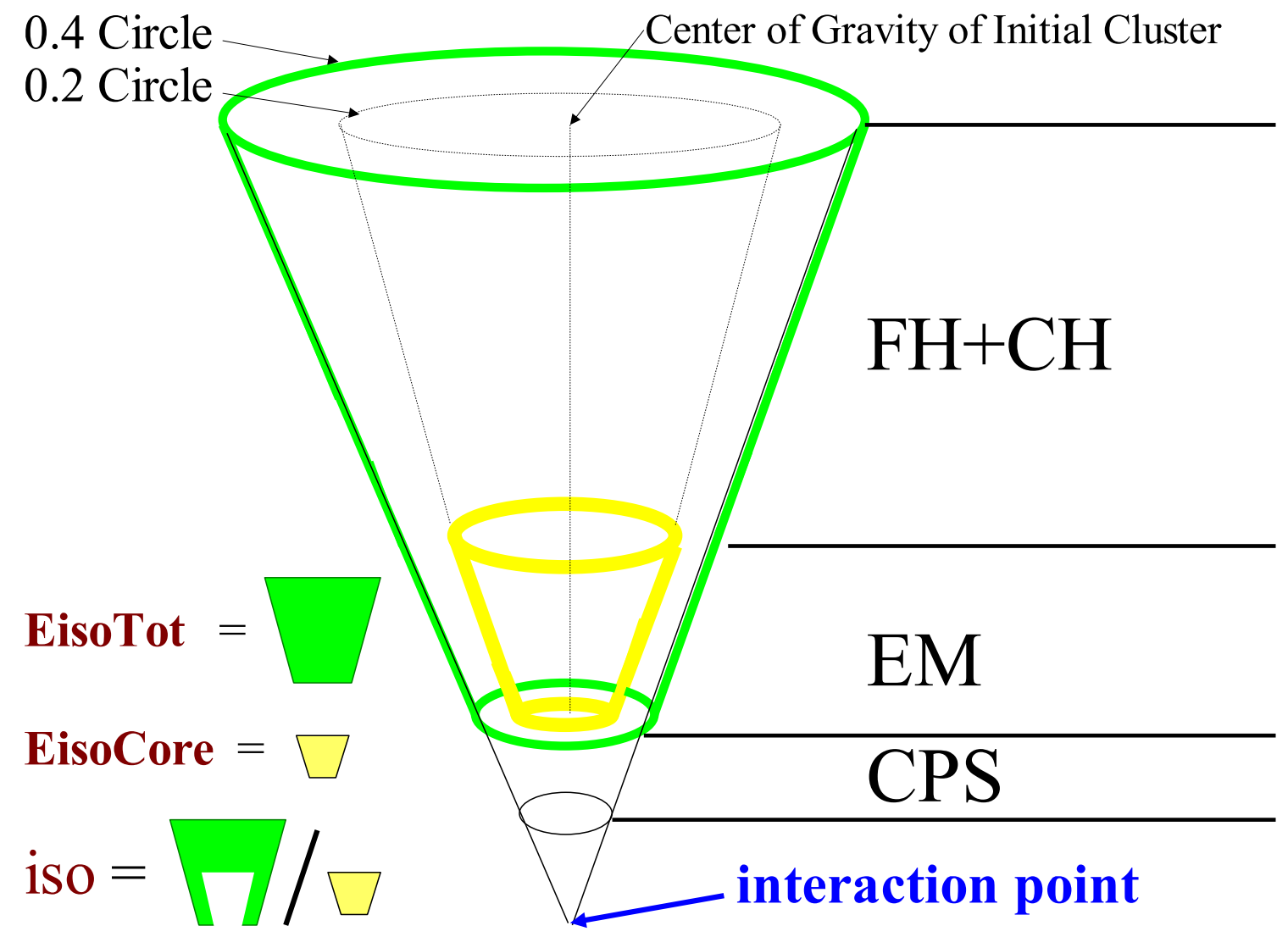

Figure 3.2: The electromagnetic (EM) isolation variable (iso) in the calorimeter. The energy measured in the central preshower (CPS) detector is not used in the calculation. Energy measured in the EM, fine hadronic $(\mathrm{FH})$, and coarse hadronic $(\mathrm{CH})$ layers are used in the calculation. Diagram from [41].

EisoTot is the total energy in the cone with radius $R=\sqrt{(\Delta \eta)^{2}+(\Delta \phi)^{2}}=0.4$ centered on the highest $\mathrm{E}_{\mathrm{T}}$ tower in the cluster, and EisoCore is the energy in the EM layers of a cone of radius 0.2 with the same center (see Figure 3.2). The isolation must be less than 0.2. Clusters which pass these cuts are saved as EM clusters.

\subsection{Missing Transverse Energy Reconstruction}

Missing transverse energy (MET) is a useful tool to select events containing particles which will not interact with the detector [42]. Its calculation requires information from the tracking system, calorimeter, and muon system. 
MET is calculated by first summing over the $i$ cells in the calorimeter

$$
\mathrm{E}_{\mathrm{x}, \mathrm{y}}^{\mathrm{vis}}=\sum_{\text {cells }} \mathrm{E}_{\mathrm{i}}^{\mathrm{x}, \mathrm{y}}
$$

to determine the $\mathrm{x}$ - and $\mathrm{y}$-components of the visible energy, $\mathrm{E}^{\mathrm{vis}}$, in the calorimeter. The $\mathrm{x}$ - and $\mathrm{y}$-projections are $\mathrm{MET}_{\mathrm{x}}=-\mathrm{E}_{\mathrm{x}}^{\mathrm{vis}}$ and $\mathrm{MET}_{\mathrm{y}}=-\mathrm{E}_{\mathrm{y}}^{\mathrm{vis}}$. The resultant MET is thus $\mathrm{MET}=\sqrt{\left(\mathrm{MET}_{\mathrm{x}}\right)^{2}+\left(\mathrm{MET}_{\mathrm{y}}\right)^{2}}$.

The MET can be corrected for muons by subtracting the $\mathrm{x}$ - and $\mathrm{y}$-components of the muon momenta from the $\mathrm{x}$ - and $\mathrm{y}$-projections of the MET. The MET corrected for muons, $\mathrm{MET}^{\mathrm{CAL}+\mathrm{MUON}}$, is calculated as

$$
\mathrm{MET}^{\mathrm{CAL}+\mathrm{MUON}}=\sqrt{\left(\mathrm{MET}_{\mathrm{x}}-\mathrm{p}_{\mathrm{x}}^{\text {muons }}\right)^{2}+\left(\mathrm{MET}_{\mathrm{y}}-\mathrm{p}_{\mathrm{y}}^{\text {muons }}\right)^{2}}
$$

where $\mathrm{p}_{\mathrm{x}}^{\text {muons }}$ and $\mathrm{p}_{\mathrm{y}}^{\text {muons }}$ are the summed $\mathrm{x}$ - and $\mathrm{y}$-components of the muon momenta. 


\section{Chapter 4}

\section{Analysis}

Supersymmetry (SUSY) is a proposed symmetry between fermions and bosons (see

Chapter 1). In this chapter, the details of the search for evidence of supersymmetry are presented, including calculations of trigger and reconstruction efficiencies, modeling of backgrounds, and development of cuts to separate signal from background.

This analysis searches for trilepton events, which may be evidence for supersymmetry, indirectly by searching for like-sign muon pairs. It has been suggested that the reach into some parts of SUSY parameter space will be greater when searching with the like-sign dilepton final state than the trilepton final state [43].

\subsection{Data Set}

The data used here was recorded between August 2002 and April 2004. It has been reconstructed with p14.x versions of the dØreco package. The data sample used is the DØ New Phenomena group dimuon skim [44], provided by the DØ common samples group [45]. The data sample is composed of events with two loose muons and no trigger requirement.

The quality of the data has been checked by every D $\varnothing$ subdetector group. Each 
group provides a list of runs which are marked bad due to problems with a particular subdetector. Events in the sample from runs marked as bad by the muon, Jet/MET, SMT, or CFT groups are removed. Luminosity blocks marked as bad by the luminosity group or identified as bad by the calorimeter group are also removed, as these events may have mismeasured MET and isolation. Up to run 174306, the trigger 2MU_A_L2M0 is required to have fired. From run 174307 to run 184746, the trigger 2MU_A_L2ETAPHI is required to have fired. For data after run 185746, either 2MU_A_L2M0_TRK5 or 2MU_A_L2M0_L3L6 is required to have fired (see Section 4.2 for trigger definitions). After removing bad runs and luminosity blocks, the total integrated luminosity of the data sample that passes one of the selected triggers is $239 \pm 16 \mathrm{pb}^{-1}$.

\subsubsection{Muon Selection Criteria}

Reconstructed muons must satisfy the following criteria (see [38] for more information about certified muons):

- Satisfy at least the loose muon quality criteria specified by the muon identification group.

- Pass the following cosmic-ray-removal cuts:

1. Time between the expected arrival of a muon and the time measured in the A-layer scintillator is between -10 and $10 \mathrm{~ns}$ (Cosmic-ray muons will be measured as "out-of-time" with respect to the hard interaction in the event).

2. Time between the expected arrival of a muon and the time measured in the B- or C-layer scintillator is between -10 and $10 \mathrm{~ns}$.

3. Measured distance of closest approach to the primary vertex is less than 
$0.16 \mathrm{~cm}$. (Cosmic-ray muons can come from any direction, and thus will not follow paths that point back to the primary vertex.)

- Parameter $n s e g$ is equal to 3 (the muon has reconstructed segments in the A and BC layers, plus a match to a central track).

- Be matched to a three-dimensional central track (two-dimensional tracks based on only the axial layers of the tracking detectors are also available).

- Isolated according to the definition in Section 3.2.2. (There is a large dimuon background from $\mathrm{b} \overline{\mathrm{b}}$ production. This cut serves to remove events containing these muons. Events produced in decays of supersymmetric particles should be produced in decays not involving jets, and hence be isolated.)

\subsection{Trigger Efficiency}

The triggers used in collecting data are called 2MU_A_L2M0, 2MU_A_L2ETAPHI, 2MU_A_L2M0_TRK5, and 2MU_A_L2M0_L3L6. They have the same requirement at L1; at least two muons found in the event using only the scintillating tiles.

At L2, the triggers differ. 2MU_A_L2M0 requires one L2 medium muon, while 2MU_A_L2ETAPHI requires two L2 medium muons separated by at least 0.15 in $\eta$ or 0.24 in $\phi$. L2 medium muons are required to have at least one hit in the A layer, and one hit in the $\mathrm{B}$ or $\mathrm{C}$ layer. The separation requirement is imposed to avoid triggering on a single muon which the L2 trigger system considers incorrectly to be two muons very close together.

Only 2MU_A_L2M0_TRK5 and 2MU_A_L2M0_L3L6 have a L3 trigger requirement. The L1 and L2 requirement for both is the same as the 2MU_A_L2M0 trigger. At L3, 2MU_A_L2M0_TRK5 requires one track with $\mathrm{p}_{\mathrm{T}}>5 \mathrm{GeV} / \mathrm{c}$ and 2MU_A_L2M0_L3L6 requires one muon with $\mathrm{p}_{\mathrm{T}}>6 \mathrm{GeV} / \mathrm{c}$. 
The L1, L2, and L3 per-muon trigger efficiencies are calculated with respect to reconstructed muons, as defined in Section 4.1.1.

\subsubsection{L1 Muon Trigger Efficiency}

To calculate the L1 muon efficiency with respect to reconstructed muons, events containing reconstructed muons must be used. Trigger objects stored in the data can be checked against the reconstructed muons to calculate the trigger efficiency. However, any event chosen has already fired a trigger in order to be written to tape, and care must be taken to avoid a bias in calculating the muon trigger efficiency.

To measure the trigger efficiency, dimuon events that fired a single-muon trigger are selected. At least one of the muons in each dimuon event must have been detected at the trigger level and caused the trigger to fire. The second muon may or may not have been detected at the trigger level, and can be used to study the trigger efficiency without bias.

In each dimuon event, a "control muon" is chosen using a random number generator. If the control muon is in the same detector octant as a L1 trigger object, the second muon is used as an unbiased "test muon." It can be determined whether the test muon has a matching L1 muon object. The percentage of test muons with matching L1 objects is the per-muon L1 efficiency. The per-muon L1 efficiency can be calculated as a function of the reconstructed muon $\mathrm{p}_{\mathrm{T}}, \eta$, and $\phi$.

Figure 4.1a shows the L1 muon efficiency as a function of the reconstructed muon

$\mathrm{p}_{\mathrm{T}}$ measured with respect to loose, $n s e g=3$ (track-matched) muons. The efficiency versus $\eta$ is plotted in Figure $4.1 \mathrm{~b}$ for muons with $\mathrm{p}_{\mathrm{T}}$ greater than $5 \mathrm{GeV} / \mathrm{c}$. 


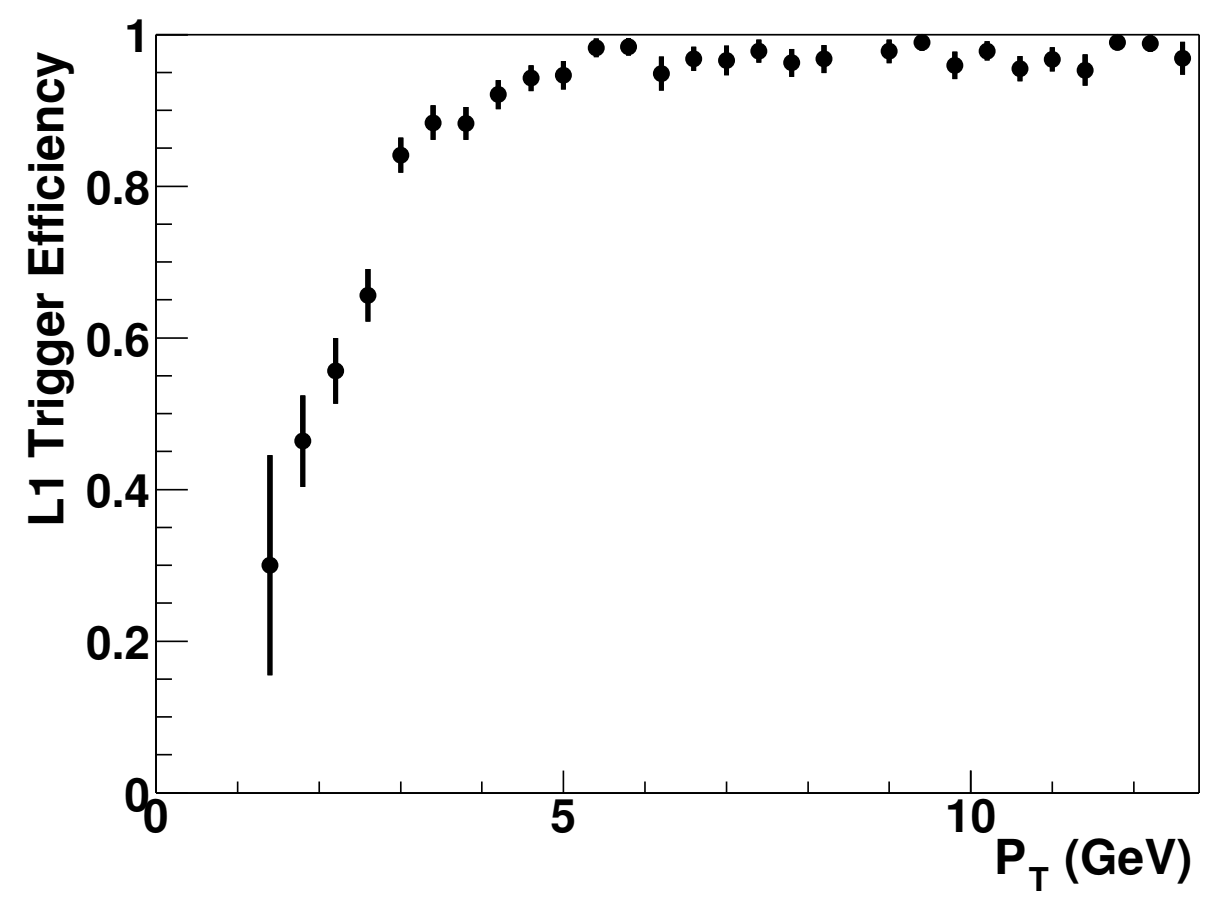

(a)

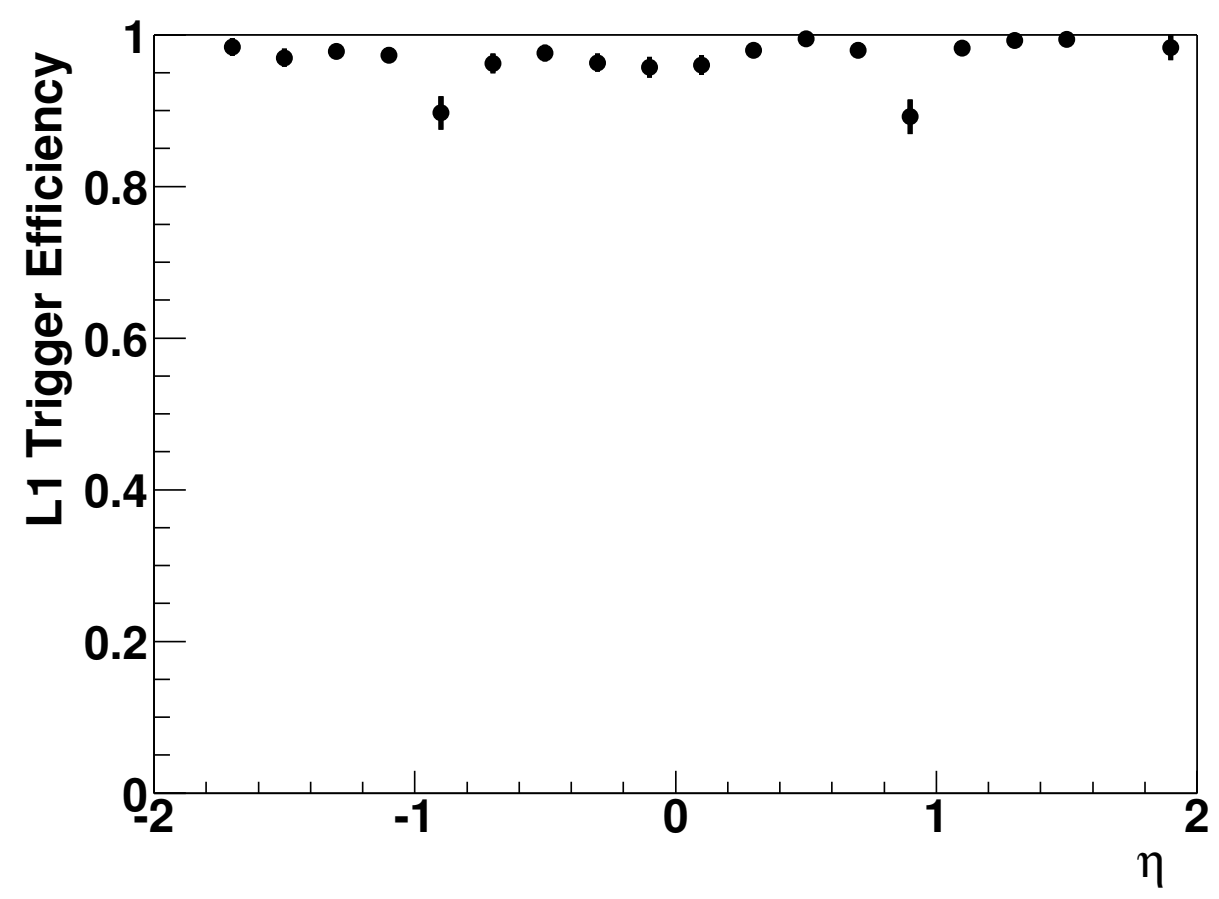

(b)

Figure 4.1: a) The L1 trigger efficiency as a function of $\mathrm{p}_{\mathrm{T}}$ with respect to loose, $n s e g=3$ muons. b) The L1 trigger efficiency as a function of $\eta$ with respect to loose, $n s e g=3$ muons with $\mathrm{p}_{\mathrm{T}}$ greater than $5 \mathrm{GeV} / \mathrm{c}$. 


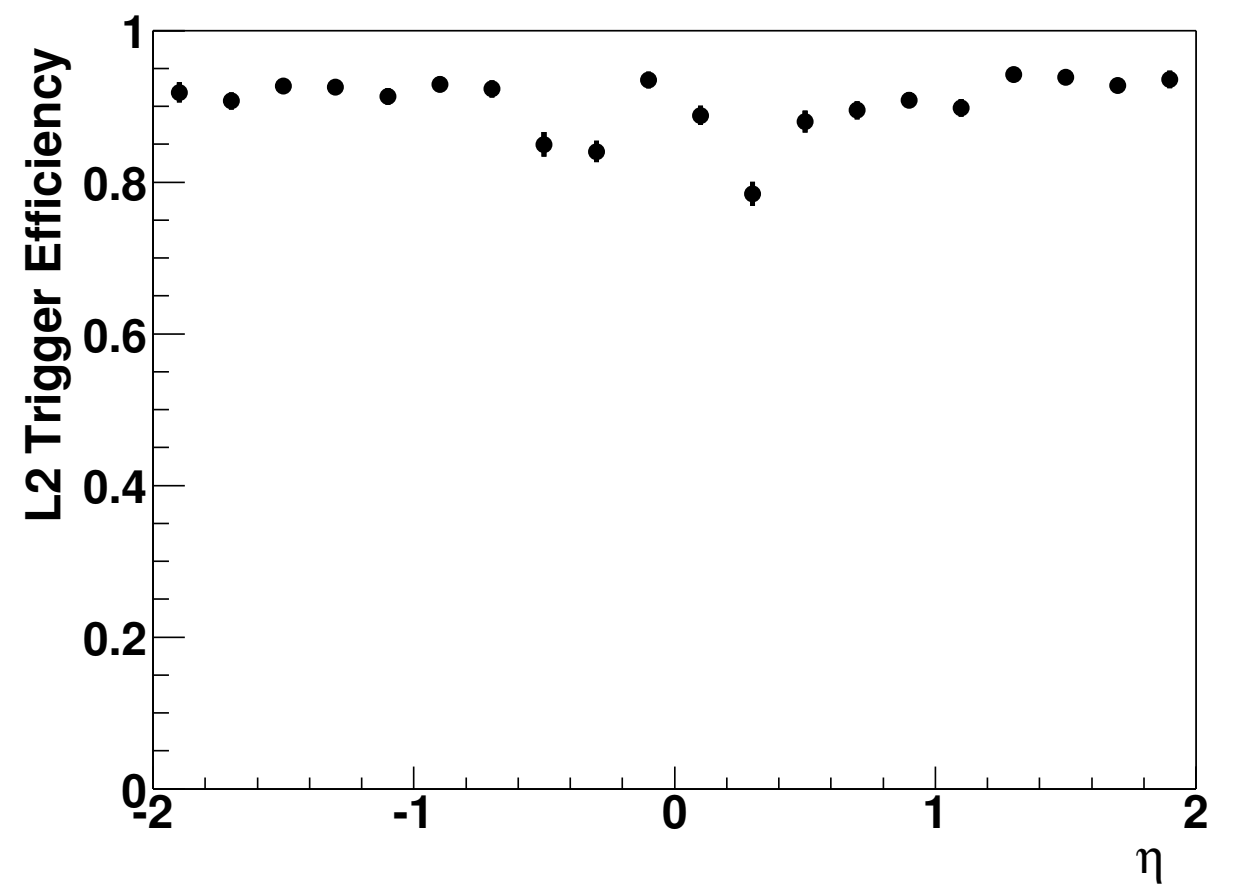

(a)

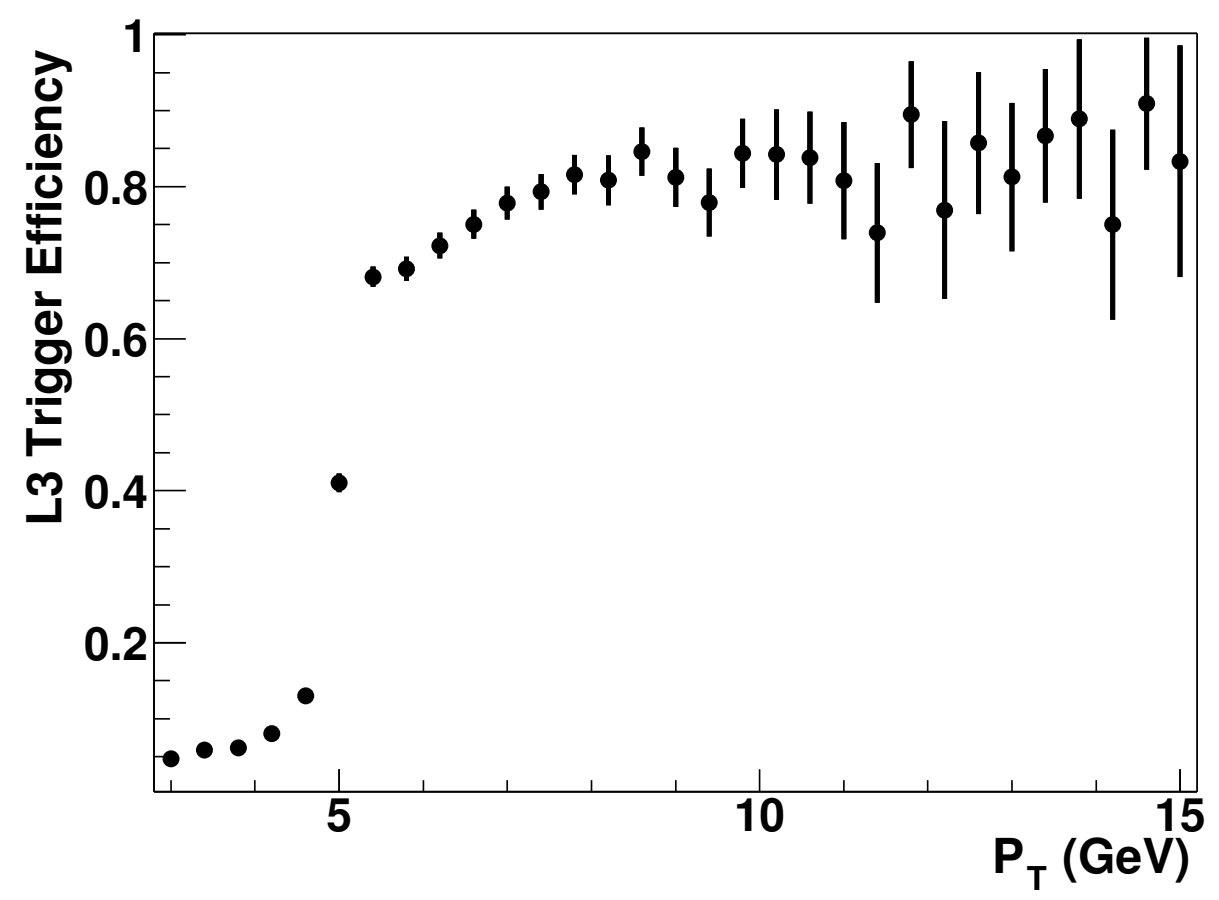

(b)

Figure 4.2: a) The L2 trigger efficiency as a function of $\eta$ with respect to loose, $n s e g=3$ muons with $\mathrm{p}_{\mathrm{T}}$ greater than $5 \mathrm{GeV} / \mathrm{c}$. b) The L3 trigger efficiency as a function of $\mathrm{p}_{\mathrm{T}}$ with respect to loose, $n s e g=3$ muons. 


\subsubsection{L2 Muon Trigger Efficiency}

The method used to measure the L2 trigger efficiency is similar to the method used to measure the L1 muon trigger efficiency. Dimuon events that pass the 2MU_A_L2M0 trigger are used to measure the L2 trigger efficiency. Both muons must be matched to L1 muons. A control muon is chosen randomly, and is required to be matched to a L2 muon within $\Delta \mathrm{R}<0.5$. The other muon becomes the test muon, and can be checked for a L2 muon match to determine the L2 trigger efficiency. The per-muon L2 trigger efficiency can be measured with respect to reconstructed muons. The L2 trigger efficiency versus $\eta$ is plotted in Figure 4.2a.

\subsubsection{L3 Muon Trigger Efficiency}

The method used to measure the L3 trigger efficiency is similar to the method used to measure the L2 trigger efficiency. Dimuon events that pass the trigger 2MU_A_L2M0 are used to measure the L3 trigger efficiency. Both muons must be matched to L1 and L2 muons. A control muon is chosen randomly. The other muon becomes the test muon, and can be checked for a L3 muon or track match to determine the L3 efficiency. The per-muon L3 efficiency can be measured with respect to reconstructed muons. The L3 efficiency versus $\mathrm{p}_{\mathrm{T}}$ is plotted in Figure $4.2 \mathrm{~b}$.

\subsubsection{Total Trigger Efficiency}

The L1, L2, and L3 trigger efficiencies are applied to all Monte Carlo samples by weighting the events by the trigger efficiencies. Since three different triggers were used, the total efficiencies are slightly different for the three run ranges. The three efficiencies are applied in proportion to the amount of data collected with each trigger. 


\subsection{Reconstruction Efficiencies and Smearing in Data and Monte Carlo}

The measured muon $\mathrm{p}_{\mathrm{T}}$ resolutions in data and Monte Carlo samples do not agree [46]. The momentum resolution in the Monte Carlo is better than in the data. To bring the Monte Carlo momentum resolution into agreement with the data, the momentum for each Monte Carlo muon can be randomly altered, or "smeared", according to a formula determined by comparing the muon momentum resolution in Monte Carlo and data samples. Once the smearing is applied, the discrepancy between data and Monte Carlo reconstruction efficiency must be determined so that the Monte Carlo can be corrected and used to simulate the data properly.

The muon reconstruction efficiency must also be measured. While the per-muon trigger efficiency can be determined with respect to reconstructed muons, there is no analogous reference muon against which the reconstruction efficiency can be measured. The total muon reconstruction efficiency can be measured, however, by separating it into the product of the local muon efficiency and the tracking efficiency.

\subsubsection{Monte Carlo Momentum Smearing}

In order to correct the difference between the momentum resolution in Monte Carlo and data, the Monte Carlo $\mathrm{p}_{\mathrm{T}}$ is smeared according to the prescription

$$
\frac{1}{\mathrm{p}_{\mathrm{T}}} \rightarrow \frac{\mathrm{A}}{\mathrm{p}_{\mathrm{T}}}+\mathrm{f} \mathrm{G},
$$

where $\mathrm{A}=1.0, \mathrm{f}=0.002$, and $\mathrm{G}$ is a random number from a Gaussian distribution with width one and mean zero. The smearing values were determined by comparing Z bosons in Monte Carlo and data samples [46]. 


\subsubsection{Tracking Efficiency}

The tracking efficiency for muons is calculated by selecting a high-purity dimuon sample. Dimuon events that fired one of the dimuon triggers are chosen. One muon, required to have a matched track, and to be matched to L1 and L2 muons, is chosen randomly to be the control muon. The other muon is the test muon, and the invariant mass of the muon pair is then formed using only the local muon system information. Pairs are chosen whose invariant mass is in the $\mathrm{J} / \Psi$ region $\left(2.0-4.0 \mathrm{GeV} / \mathrm{c}^{2}\right)$, where the number of real muons should dominate the number of muons from backgrounds. The frequency with which tracks are matched to the local muons is measured. Figures $4.3 \mathrm{a}$ and 4.3b show the tracking efficiency as a function of $\eta$ in data and Monte Carlo. Figure 4.4 shows the ratio of these efficiencies.

\subsubsection{Local Muon Efficiency}

A sample composed of one track and one muon matched to a track, a L1 muon, and a L2 muon is selected. The invariant mass of the pair of particles is required to be

in the $\mathrm{Z}$ region $\left(70 \mathrm{GeV} / \mathrm{c}^{2}<\right.$ mass $\left.<110 \mathrm{GeV} / \mathrm{c}^{2}\right)$, and the efficiency is measured by recording how many tracks have matching muons. The single muon skim [45] is used to avoid a bias from using the dimuon skim. A single muon trigger is required to have fired. Figures $4.5 \mathrm{a}$ and $4.5 \mathrm{~b}$ show the loose muon efficiency as a function of $\eta$ in data and Monte Carlo. Figure 4.6 shows the ratio of these efficiencies. A correction is applied to the Monte Carlo muons to remove the discrepancy.

\subsubsection{Muon Reconstruction Efficiency}

The muon reconstruction efficiency is obtained by multiplying the reconstruction and tracking efficiencies. The muon reconstruction efficiency is plotted as a function of $\eta$ in Figure 4.7. 


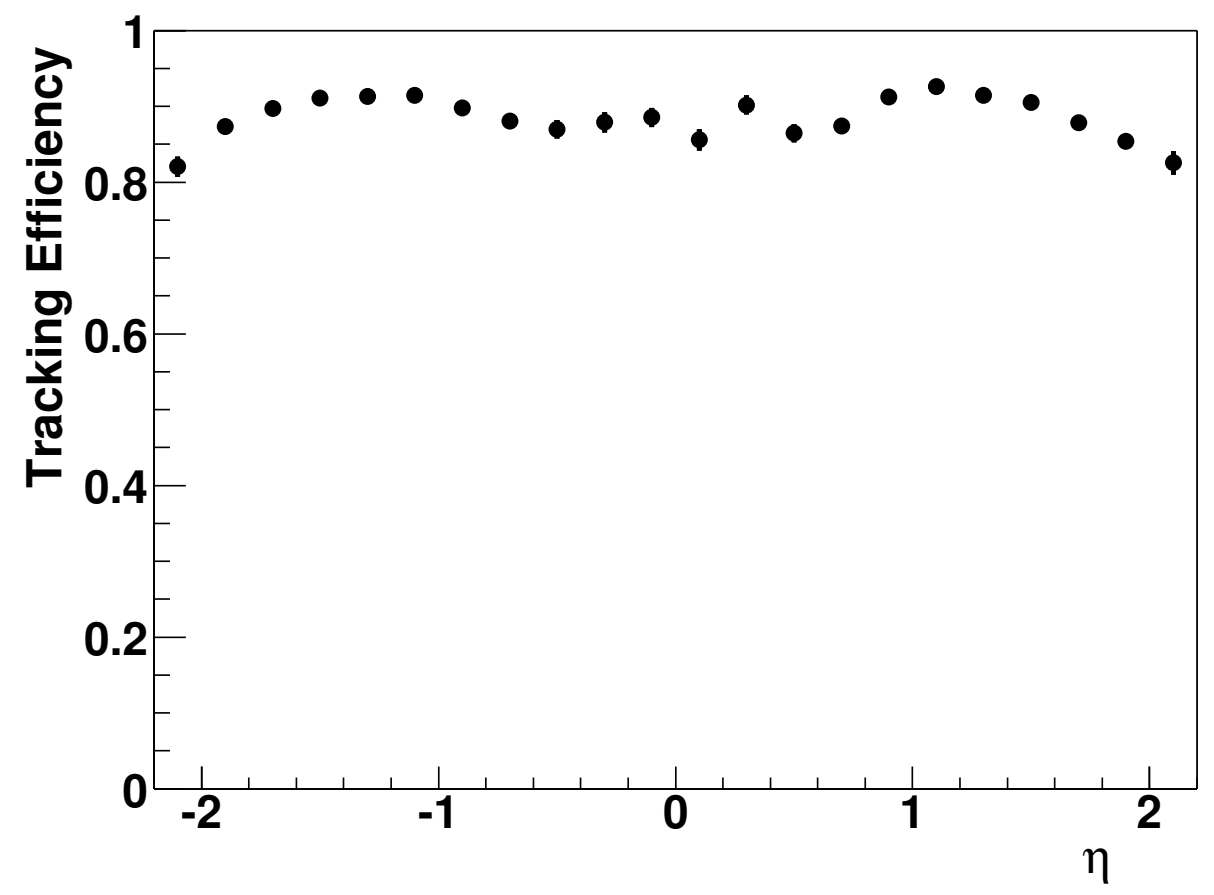

(a)

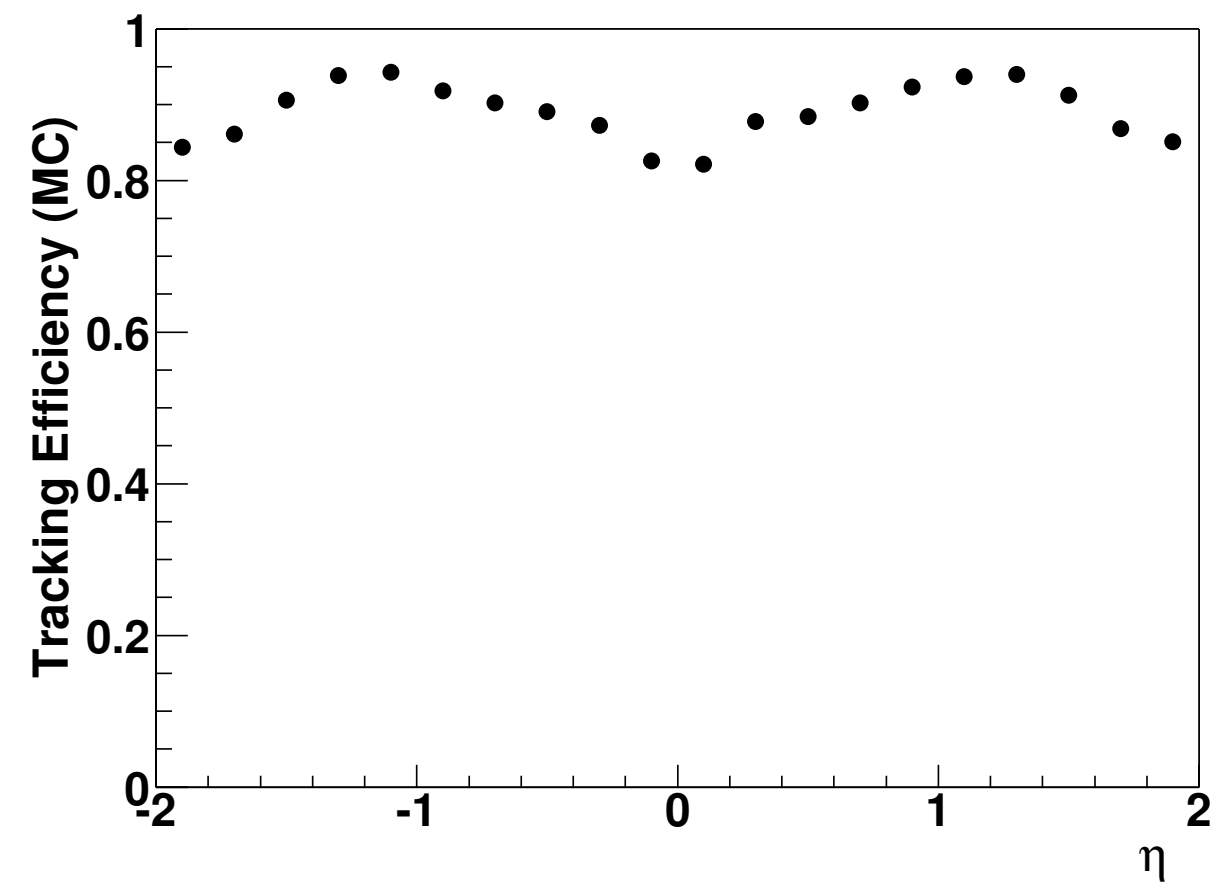

(b)

Figure 4.3: a) Tracking efficiency as a function of $\eta$ with respect to local muons in the $\mathrm{J} / \Psi$ peak measured in data. b) Tracking efficiency of reconstructed muons as a function of $\eta$ with respect to Monte Carlo (MC) muons. 


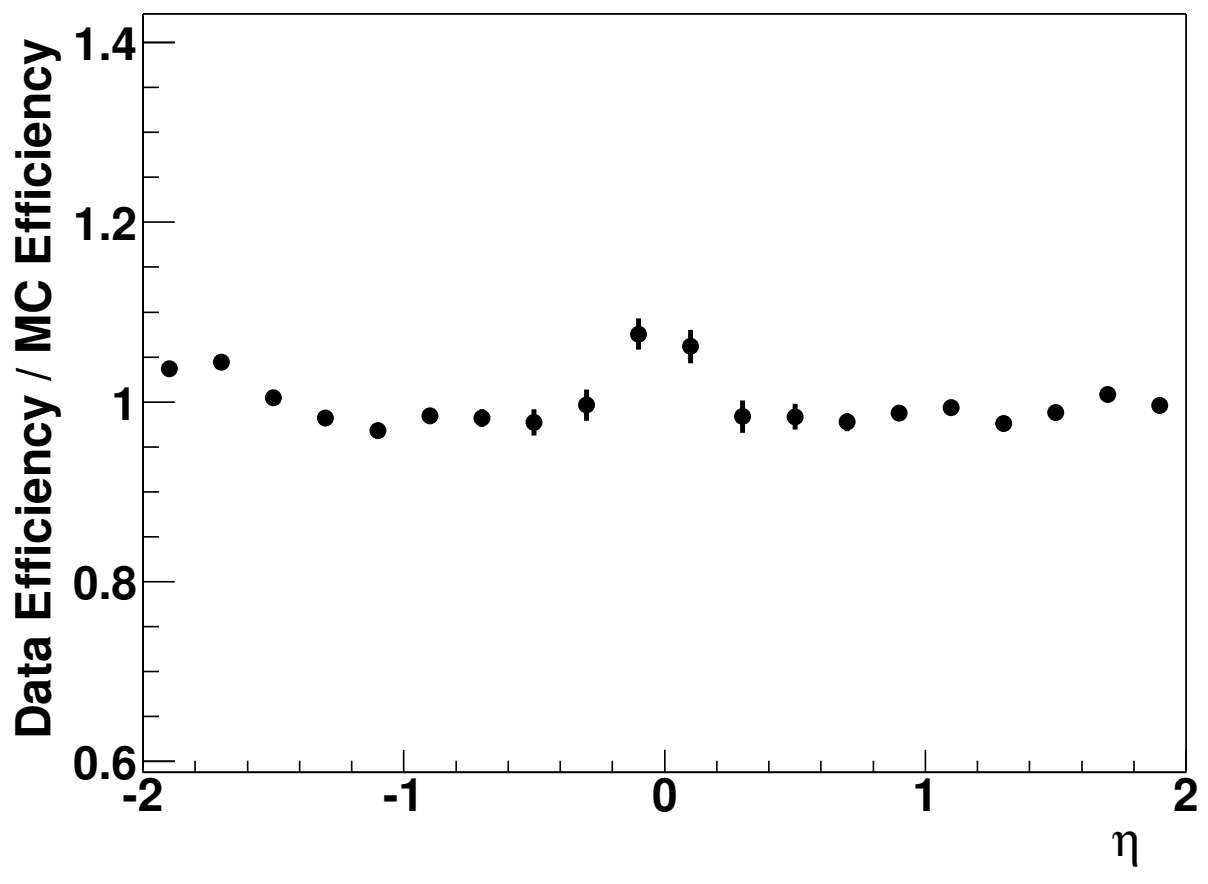

Figure 4.4: Tracking efficiency measured in data divided by the Monte Carlo (MC) tracking efficiency as a function of $\eta$ with respect to local muons in the $\mathrm{J} / \Psi$ peak.

There is relatively good agreement between the reconstruction efficiency as measured in Monte Carlo and data samples (see Figure 4.7). The small difference is removed by weighting the Monte Carlo by the ratio of the efficiency in the data to the efficiency in the Monte Carlo.

\subsection{Backgrounds}

Not many Standard Model processes are capable of generating a pair of isolated like-sign muons. The background processes considered are $t \bar{t}, b \bar{b} / c \bar{c}, W+j e t s$, and diboson (WZ and ZZ) production. These backgrounds are modeled with Monte Carlo samples, with the exception of the $\mathrm{b} \bar{b} / \mathrm{c} \overline{\mathrm{c}}$ sample (see Section 4.4.1). The PYTHIA event generator [47] and the full DØ simulation and reconstruction software chain are used for the Monte Carlo samples with the exception of $\mathrm{W}+\mathrm{jets}$, for which the ALPGEN event generator [48] is used. For W+jets events, the ALPGEN generator 


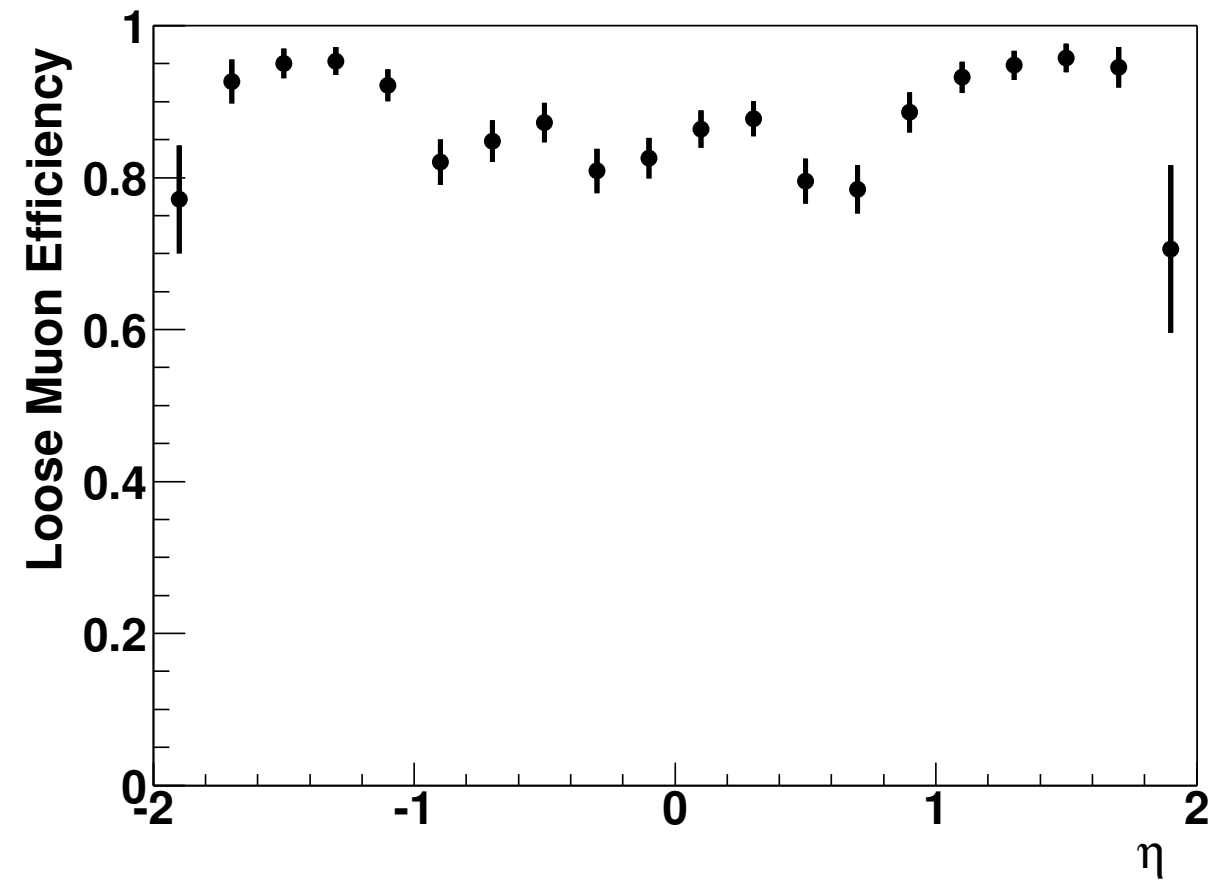

(a)

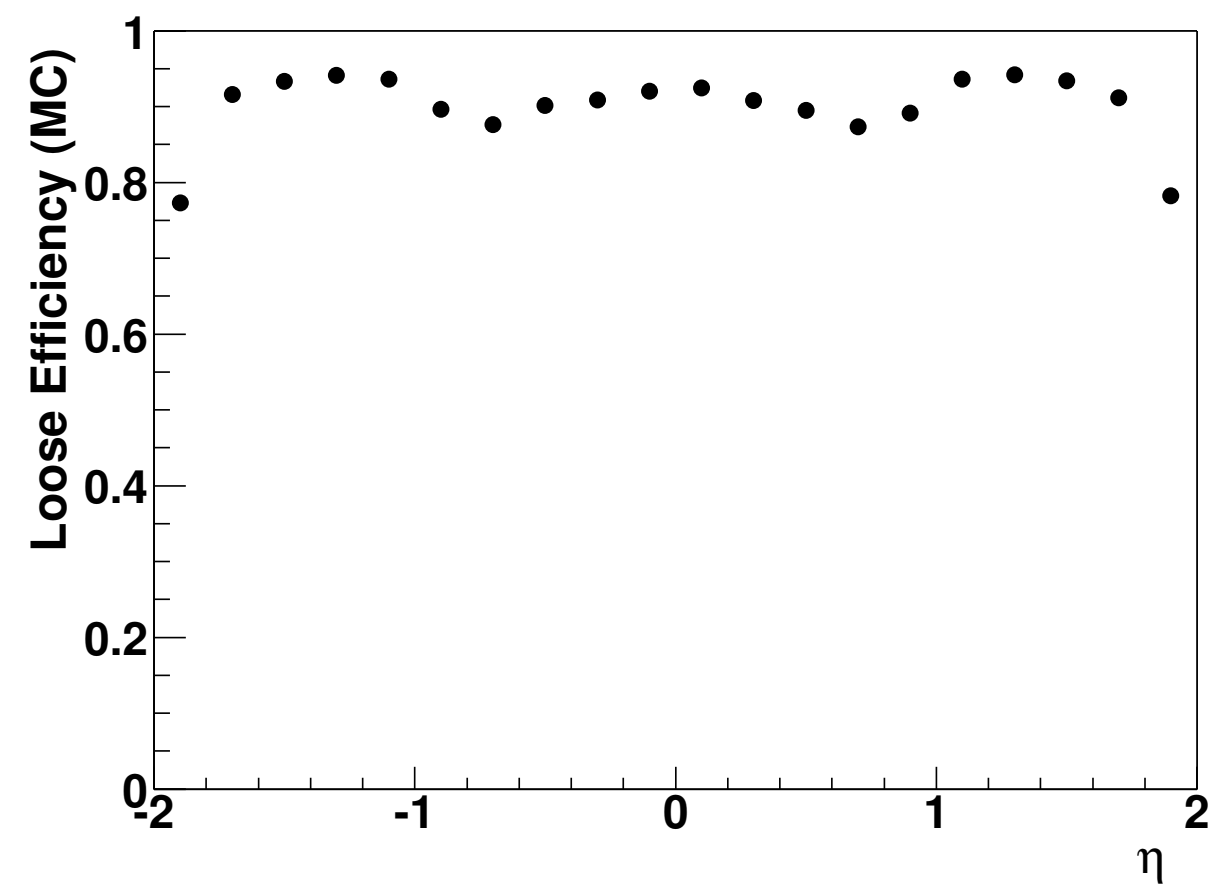

(b)

Figure 4.5: a) The loose muon efficiency as a function of $\eta$ with respect to tracks measured in data. The tracks are from muon-track pairs with an invariant mass that lies under the $\mathrm{Z}$ peak. b) The loose muon efficiency as a function of $\eta$ with respect to Monte Carlo (MC) muons. 


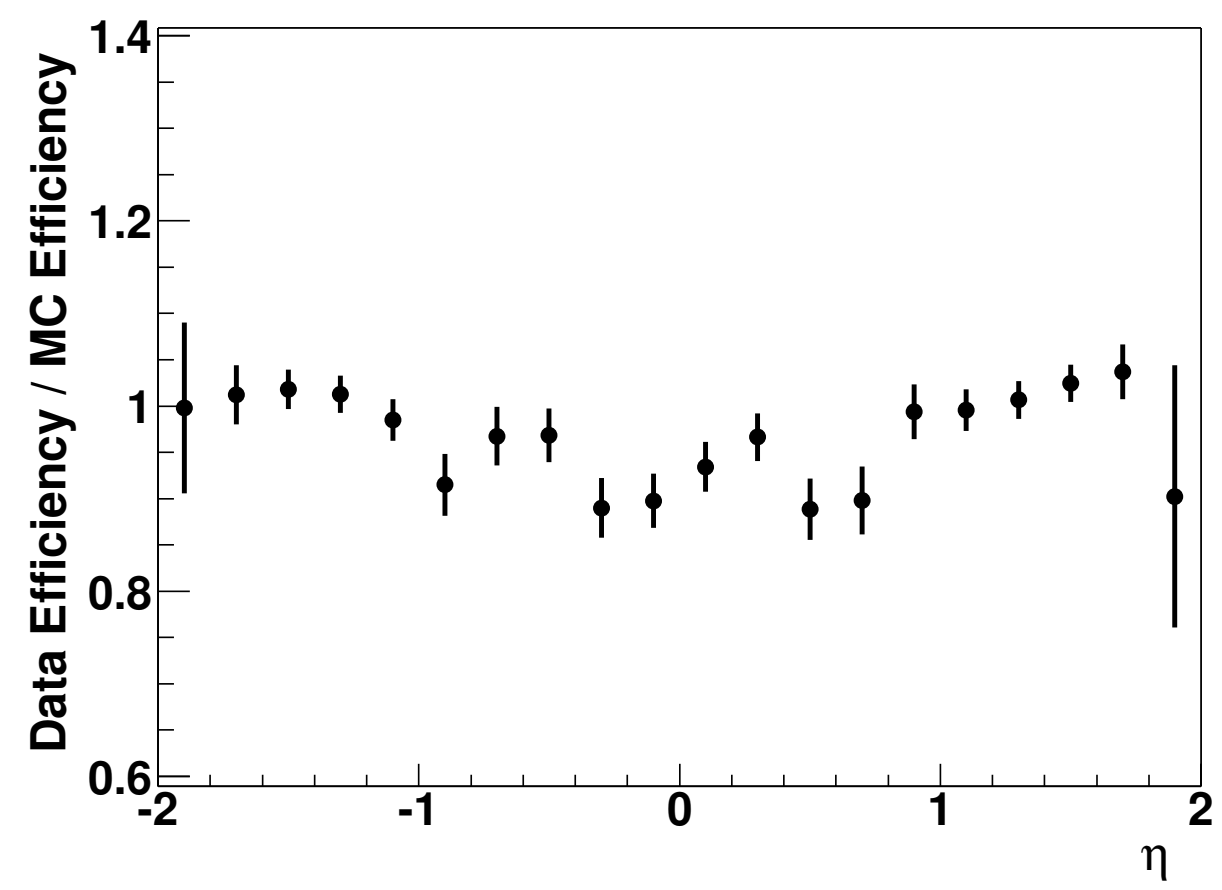

Figure 4.6: The loose muon efficiency as measured in data divided by the Monte Carlo (MC) local muon efficiency, as a function of $\eta$ with respect to local muons in the Z peak.

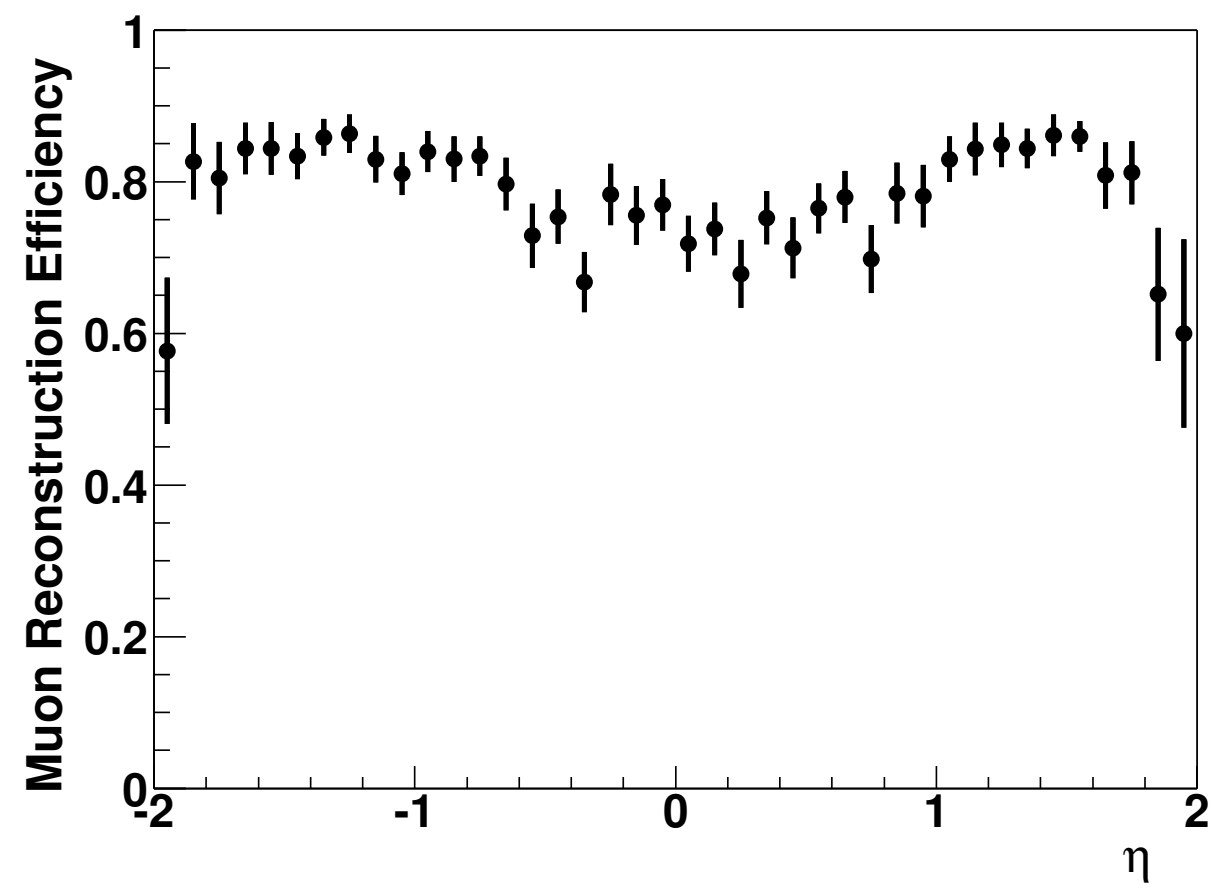

Figure 4.7: The total muon reconstruction efficiency as a function of $\eta$. 


\begin{tabular}{cccc}
\hline Sample & $\begin{array}{c}\text { MC Cross } \\
\text { Section }(\mathrm{pb})\end{array}$ & $\begin{array}{c}\text { Number of } \\
\text { MC Events }\end{array}$ & $\begin{array}{c}\text { Integrated } \\
\text { Luminosity }\left(\mathrm{pb}^{-1}\right)\end{array}$ \\
\hline $\mathrm{t} \overline{\mathrm{t}}$ & 0.07 & 1250 & 18382 \\
$\mathrm{~W}+1$ jet & 757.6 & 115000 & 151.8 \\
$\mathrm{~W}+2$ jets & 222.1 & 46000 & 207.1 \\
WZ & 3.7 & 16500 & 4459.4 \\
ZZ & 1.1 & 15000 & 13393 \\
Wbb & 1.54 & 32000 & 20779 \\
Zbb & 0.24 & 96500 & 402083 \\
$\mathrm{Z} / \gamma(15-60)$ & 113 & 49000 & 434 \\
$\mathrm{Z} / \gamma(60-130)$ & 261 & 62750 & 240 \\
$\mathrm{Z} / \gamma(130-250)$ & 1 & 22500 & 22500 \\
\hline
\end{tabular}

Table 4.1: The sizes, calculated cross sections, and integrated luminosities of the Monte Carlo (MC) samples.

is better than the PYTHIA generator. The number of jets can be counted in each of the $\mathrm{W}$ events in data samples. ALPGEN correctly predicts the number of events with each number of jets, while PYTHIA does not. The CTEQ5L parton distribution functions (PDFs) are used in generating all the Monte Carlo samples. Table 4.1 lists the Monte Carlo samples used.

The trigger and reconstruction efficiencies, described in Sections 4.2 and 4.3, are applied to the Monte Carlo samples. For the $\mathrm{b} \overline{\mathrm{b}} / \mathrm{c} \overline{\mathrm{c}}$ background, an estimate from the data is used as described in the following section.

\subsubsection{Background From $\mathrm{b} \overline{\mathrm{b}} / \mathrm{c} \overline{\mathrm{c}}$ Production}

The largest source of like-sign muon pairs is expected to be $\mathrm{b} \overline{\mathrm{b}}$ and $c \overline{\mathrm{c}}$ production. For example, like-sign muon pairs can be produced when one B meson's decay produces a muon, and the other $\mathrm{B}$ meson oscillates before decaying, producing a muon with the same sign. A cascade decay of a b quark $(b \rightarrow c \bar{c} s)$ from a b $\bar{b}$ pair can also produce a like-sign muon pair.

The ideal strategy to model this background would be to generate a Monte Carlo 


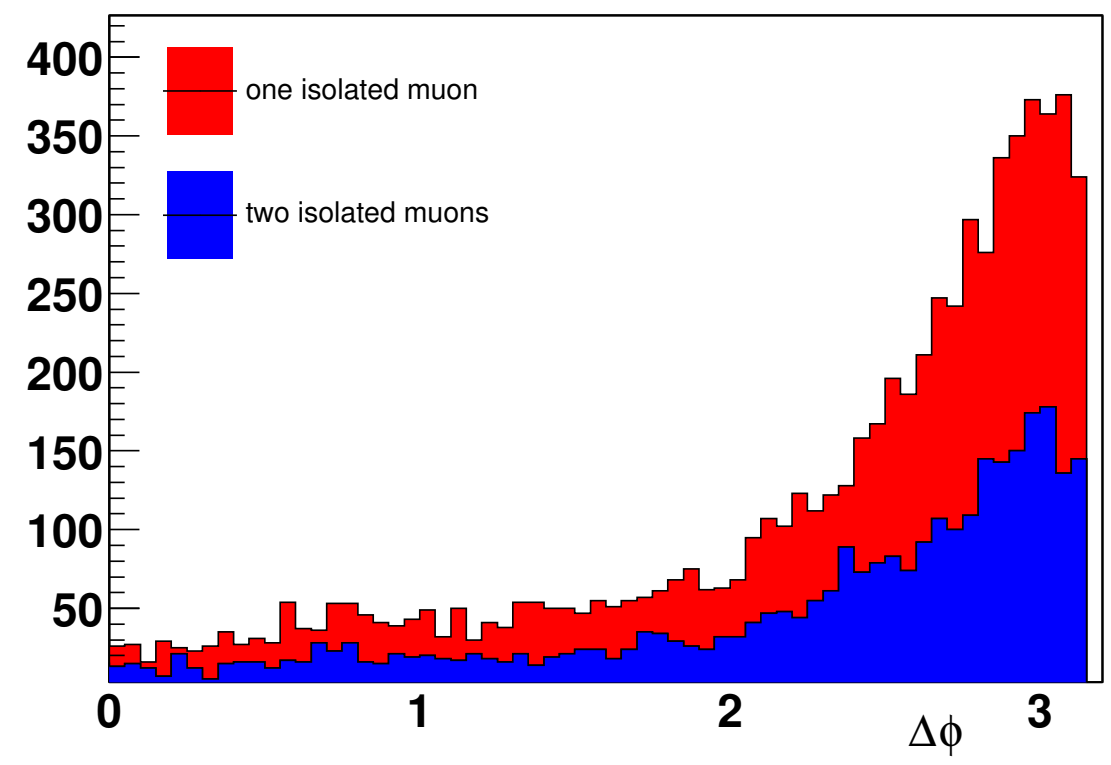

Figure 4.8: The measured $\Delta \phi$ between muons in like-sign muon pairs in the data for the cases where both muons are isolated and only one is isolated.

sample, as is done with all the other backgrounds. This technique has been tried for $\mathrm{b} \overline{\mathrm{b}} / \mathrm{c} \overline{\mathrm{c}}$, and there are several problems. First, the angular distribution plots made from the data and Monte Carlo do not agree. There are not enough events produced at low values of $\Delta \phi$ in the Monte Carlo. This is most likely due to missing b $\bar{b}$ production mechanisms such as gluon splitting into b $\bar{b}$ pairs. Second, the error on the cross section is large due to a disagreement between the measured and theoretically predicted cross sections for $\mathrm{b} \overline{\mathrm{b}}$ production [49]. Finally, the large $\mathrm{b} \overline{\mathrm{b}}$ cross section and small branching ratio into like-sign muon pairs makes the production of the desired luminosity difficult. The number of Monte Carlo events that is needed to simulate the luminosity of the collected data set is very large, and grows with the size of the data set. The production of Monte Carlo samples with this many events is very time consuming, and the computing resources of the experiment are limited. Using a small Monte Carlo sample would lead to a large error on the background estimate.

In lieu of generating a $b \bar{b}$ Monte Carlo sample, data are used to estimate the 
$\mathrm{b} \overline{\mathrm{b}}$ and $c \overline{\mathrm{c}}$ background. The method relies on collecting a set of events that contains muons similar to those that will be present in the final data sample. This collection is formed by looking for like-sign pairs of muons in which one muon passes the isolation cuts, and one muon fails the isolation cuts by a small margin. The failing muon will be "nearly" isolated. A nearly isolated muon which fails the isolation criteria by a small amount will resemble an isolated muon more closely than one that failed the criteria by a large amount. The following is the definition of "nearly" isolated:

- The muon fails at least one of the isolation criteria defined in Section 3.2.2.

- The muon's hollow cone energy is less than $7 \mathrm{GeV}$.

- The $\mathrm{p}_{\mathrm{T}}$ sum of all tracks within a cone of radius 0.5 is less than $7 \mathrm{GeV} / \mathrm{c}$.

The number of events in the nearly isolated sample must be scaled to the isolated sample to accurately predict the number of background events. This is done by defining the ratio

$$
\mathrm{R}=\left.\frac{S^{N}}{B^{N}}\right|_{\Delta \phi(\mu, \mu)>2.7}
$$

where $S^{N}$ is the number of events in the sample containing two isolated muons (signal sample), and $B^{N}$ is the number of events in the background sample. $\mathrm{R}$ is measured in the region $\Delta \phi(\mu, \mu)>2.7$ to avoid the bias of normalizing to the signal sample. The $\mathrm{b} \overline{\mathrm{b}} / \mathrm{c} \overline{\mathrm{c}}$ background is more peaked in this $\Delta \phi$ region than the signal (see Figure 4.8). The cut $\Delta \phi(\mu, \mu)<2.7$ is consequently fixed as one of the final cuts on the data.

$\mathrm{R}$ is a function of the momentum of the muons in the like-sign pairs. Since most of the events in both of the samples used to determine $\mathrm{R}$ come from $\mathrm{b} \overline{\mathrm{b}}$ decays, the difference between those measured as isolated or nearly isolated is most likely due to the momentum kick the muon gets in the decay. Lower-momentum muons may be kicked away from the b jet and hence measured as isolated more frequently 
than higher momentum muons. This happens because the lower momentum muons tend to come from lower momentum B hadrons. The decay products of the lower momentum B hadrons will naturally be further apart to conserve momentum. This effect is observed when measuring $\mathrm{R}$ as a function of $\mathrm{p}_{\mathrm{T}}$ (see Figure 4.9).

$\mathrm{R}$ is measured as a function of the $\mathrm{p}_{\mathrm{T}}$ of the nearly isolated muon by first calculating the number of events in each $\mathrm{p}_{\mathrm{T}}$ bin in the nearly isolated and in the isolated sample. $\mathrm{R}$ is then calculated in each bin as

$$
\mathrm{R}\left(\mathrm{p}_{\mathrm{T}}\right)=\left.\frac{S_{\mathrm{p}_{\mathrm{T}}}^{N}}{B_{\mathrm{p}_{\mathrm{T}}}^{N}}\right|_{\Delta \phi(\mu, \mu)>2.7}
$$

where $S_{\mathrm{p}_{\mathrm{T}}}^{N}$ is the number of muons in the signal sample at a given $\mathrm{p}_{\mathrm{T}}$, and $B_{\mathrm{p}_{\mathrm{T}}}^{N}$ is the number of muons in the background sample at a given $\mathrm{p}_{\mathrm{T}}$.

The correct way to measure $\mathrm{R}$ would be as a function of the $\mathrm{p}_{\mathrm{T}}$ of the first and second muons in the pair. The statistics are not sufficient to do this. Instead, randomly choosing a muon from the two muons in the isolated sample has been tried, as well as using both muons and dividing by two. The results are similar with both methods, and the second method is used here.

To measure $\mathrm{R}$ as a function of $\mathrm{p}_{\mathrm{T}}$, an exponential function is fit to the distribution in Figure 4.9 .

The events in the $B^{N}$ sample are then weighted, event-by-event, by the value of $\mathrm{R}$ corresponding to the $\mathrm{p}_{\mathrm{T}}$ of the nearly isolated muon belonging to the muon pair in that event. A sample of like-sign pairs remains, composed of muons which represent the isolated $\mathrm{b} \overline{\mathrm{b}} / \mathrm{c} \overline{\mathrm{c}}$ muons and scaled correctly to the $S^{N}$ sample. This weighted sample is denoted here as $B_{W}$.

Once this weighted sample is in hand, its ability to mimic isolated muons from $\mathrm{b} \overline{\mathrm{b}} / \mathrm{c} \overline{\mathrm{c}}$ is tested by looking at the opposite-sign muon pair invariant mass distribution (T). The isolated muons from $\mathrm{b} \overline{\mathrm{b}} / \mathrm{c} \overline{\mathrm{c}}$ contained in the $\mathrm{T}$ sample should be similar to 


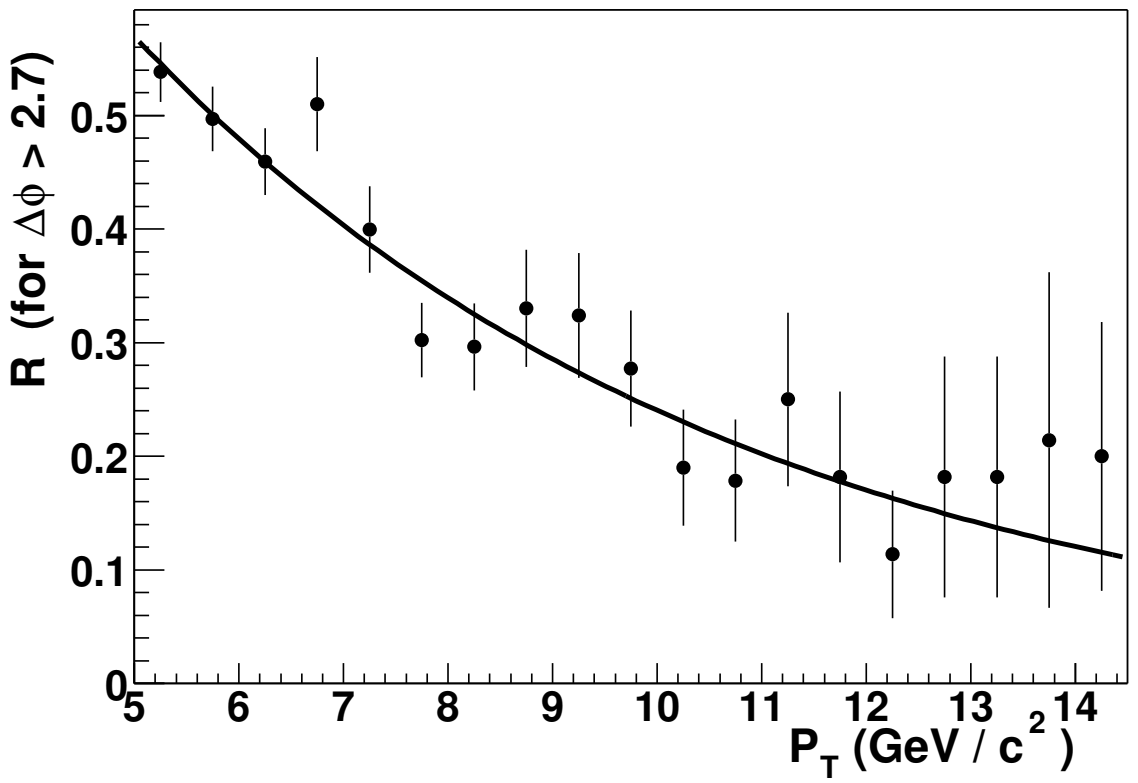

Figure 4.9: R, the ratio of the number of events in the sample with two isolated muons to the number of events in the sample with one isolated muon, as a function of the $\mathrm{p}_{\mathrm{T}}$ of the nearly isolated muon. The line is an exponential function fit to the data which was used to weight the background sample in modeling the $\mathrm{b} \overline{\mathrm{b}}$ background.

those in the $B_{W}$ sample, but the normalization will be different.

To make the comparison between the T sample and the $B_{W}$ sample, the Drell-Yan, Z, and Upsilon backgrounds as predicted by the Monte Carlo are first subtracted from the $\mathrm{T}$ sample. The opposite-sign, isolated muon pairs from $\mathrm{b} \overline{\mathrm{b}} / \mathrm{c} \overline{\mathrm{c}}$ remain $\left(T_{B}\right)$. The $B_{W}$ sample is compared to $T_{B}$. The two samples are expected to differ only in the number of events in each sample. The $B_{W}$ sample is scaled by the ratio of the number of entries remaining in the $T_{B}$ sample to the number of events in the $B_{W}$ sample, to get an estimate of the $\mathrm{b} \overline{\mathrm{b}} / \mathrm{c} \overline{\mathrm{c}}$ background in the opposite-sign distribution:

$$
B_{W}^{O S}=B_{W} \times \frac{T_{B}^{N}}{B_{W}^{N}} .
$$

$T_{B}^{N}$ is the number of events in the $T_{B}$ sample, and $B_{W}^{N}$ is the number of events in the $B_{W}$ sample. 


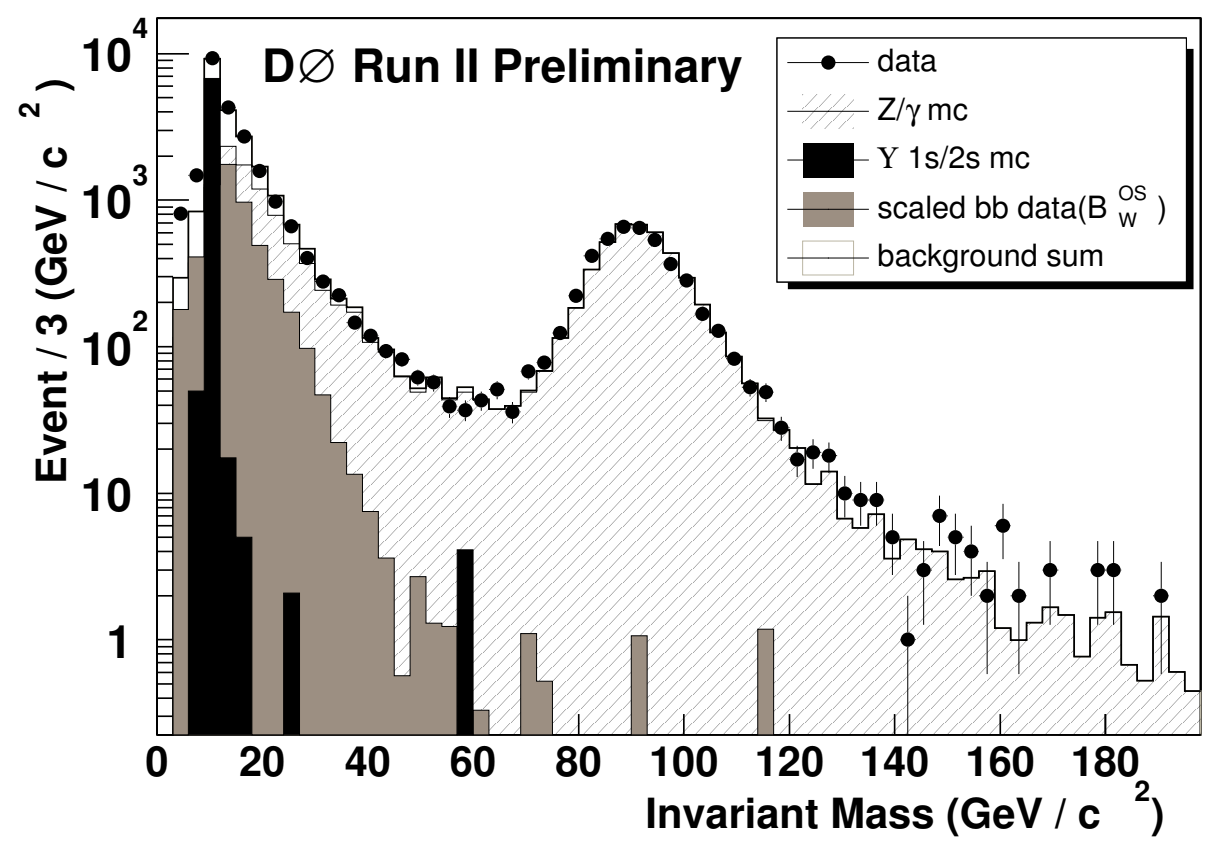

(a)

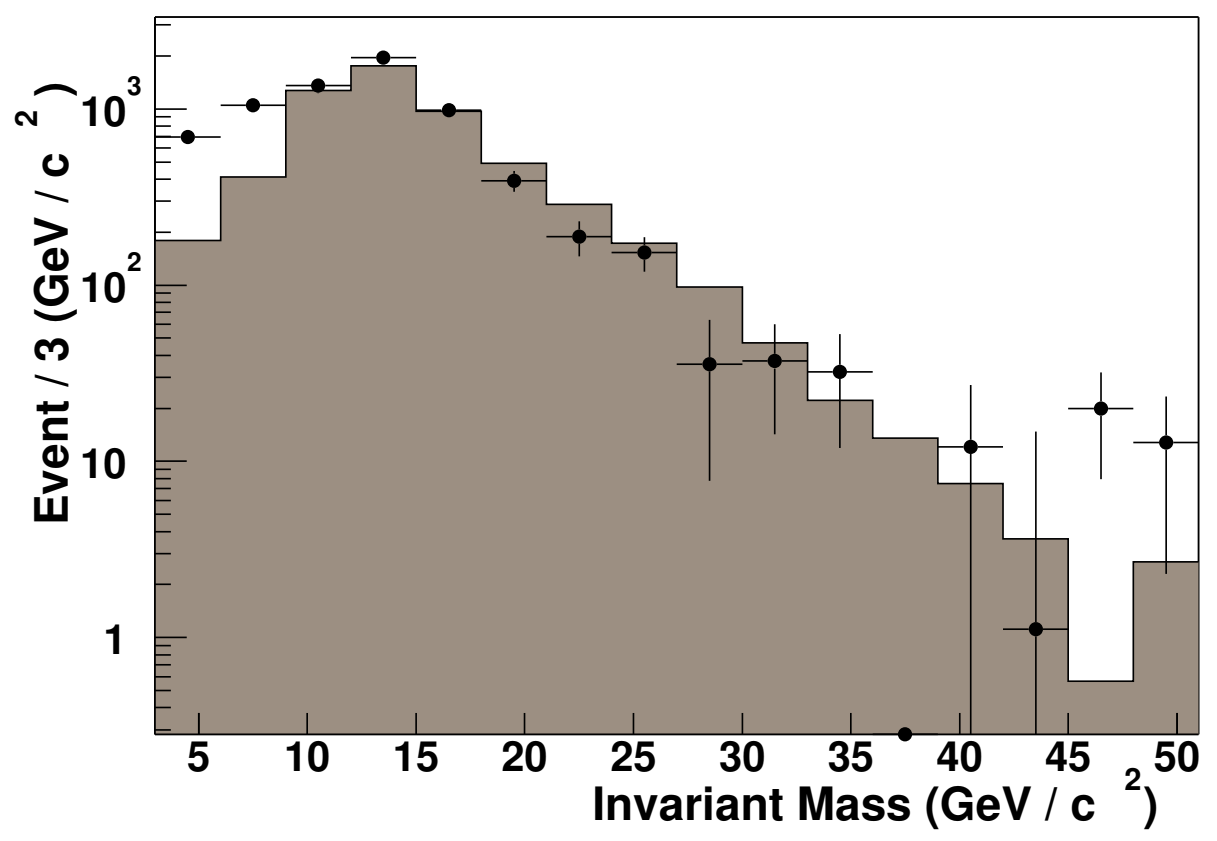

(b)

Figure 4.10: a) Circles show the invariant mass distribution of opposite-sign muon pairs. Backgrounds are described by the legend. b) Circles show the invariant mass distribution of opposite-sign muon pairs after the subtraction of Monte Carlo background estimates. The shaded histogram is the expected remaining background from $\mathrm{b} \overline{\mathrm{b}} / \mathrm{c} \overline{\mathrm{c}}$, which is estimated using like-sign data. 


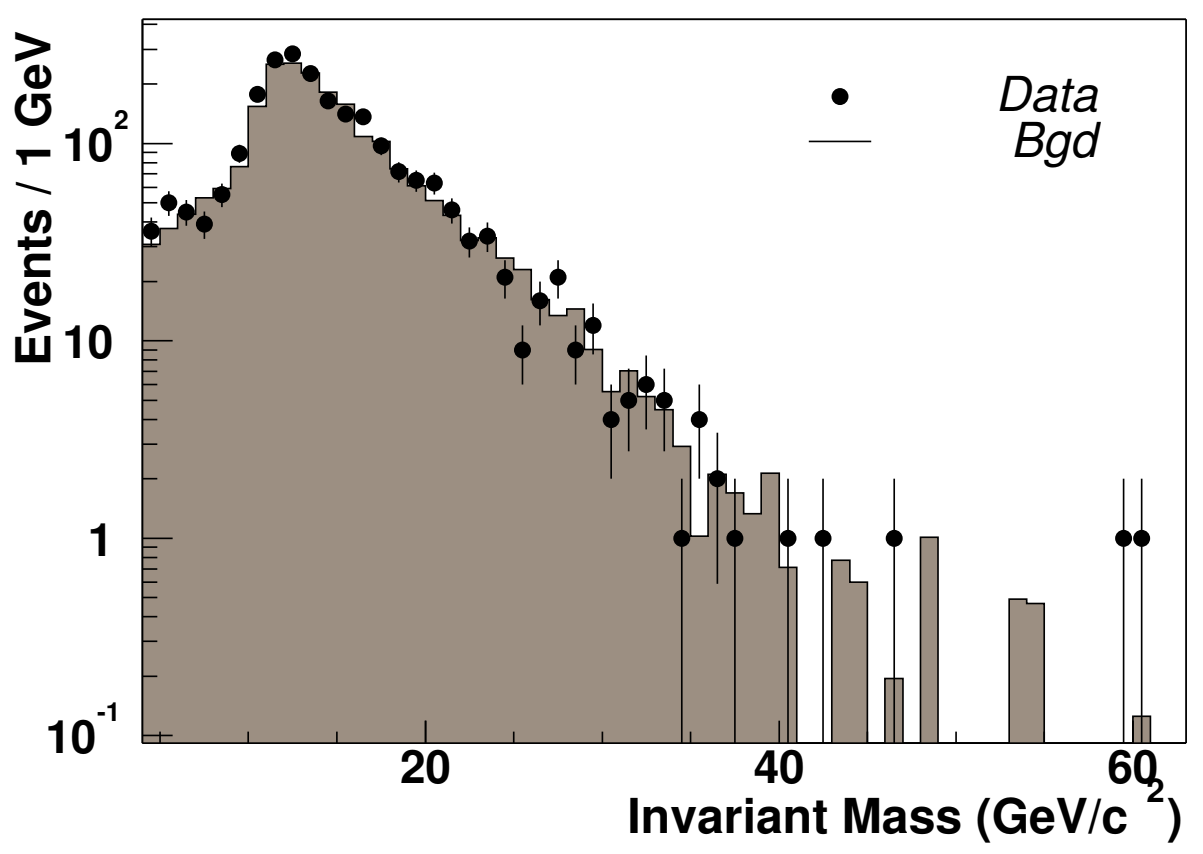

Figure 4.11: Circles show the invariant mass distribution of isolated like-sign muon pairs in data. The shaded histogram is the expected remaining background, from $\mathrm{b} \overline{\mathrm{b}} / \mathrm{c} \overline{\mathrm{c}}$, which is estimated using the nearly isolated like-sign data as described in the text.

Figure 4.10a shows the opposite-sign, isolated data and the various backgrounds. Figure $4.10 \mathrm{~b}$ shows the invariant mass distribution in data after all backgrounds except $\mathrm{b} \overline{\mathrm{b}} / \mathrm{c} \overline{\mathrm{c}}$ are subtracted, and the expected distribution from $\mathrm{b} \overline{\mathrm{b}} / \mathrm{c} \overline{\mathrm{c}}$ background. The distributions agree well except at low momentum. The difference between the data and Monte Carlo plots stems from a deficit of Monte Carlo muons just above the $\mathrm{p}_{\mathrm{T}}$ cutoff for the plot $(5 \mathrm{GeV})$. Above that, there is good agreement. The search isn't badly affected by this region since there is an $11 \mathrm{GeV} \mathrm{p}_{\mathrm{T}}$ cut on the leading muon that removes virtually all of these low mass pairs.

Using the nearly isolated sample, the $\mathrm{b} \overline{\mathrm{b}} / \mathrm{c} \overline{\mathrm{c}}$ background can be modeled in the signal sample, and the number of $\mathrm{b} \bar{b} / c \bar{c}$ events can be estimated given any set of cuts. Figure 4.11 shows the like-sign isolated data, which may contain signal, compared to the estimation from the scaled nearly isolated sample. 


\subsubsection{Sign Misidentification Background}

If the $\mathrm{p}_{\mathrm{T}}$ of a muon is badly mismeasured, the charge assigned to the muon can be wrong. This can turn a real opposite-sign pair into a measured like-sign pair. This sign misidentification happens because higher momentum tracks bend less in the inner magnetic field, so it becomes more difficult to measure their curvature. Straighter tracks are more likely to have their charge mismeasured. Since this analysis depends upon the proper determination of the sign of each muon, an estimate of the sign misidentification rate is necessary.

The major source of sign misidentification comes from $\mathrm{Z} / \gamma$ events where one muon has its momentum badly mismeasured. Backgrounds from this source as predicted by the Monte Carlo simulation are included in the background estimation in Section 4.7.

As a check of the Monte Carlo's ability to estimate the rate of sign mismeasurement, like-sign pairs with each muon having a $\mathrm{p}_{\mathrm{T}}$ greater than $15 \mathrm{GeV} / \mathrm{c}$ and with a $\phi$ difference greater than 2.8 were chosen from the Z Monte Carlo and data. These pairs should each contain a muon from a $\mathrm{Z}$ decay with its charge mismeasured. Although the statistics are low, there is reasonable agreement with 4 events observed in the data and $3.4 \pm 1.2$ events predicted in Monte Carlo studies.

\section{Sign Misidentification Background Cross-Check}

An attempt to cross-check this estimate using data was made. The spectrum of opposite-sign pairs that forms the pool of candidates for mismeasurement is plotted in Figure 4.12. This is a plot of the $\mathrm{p}_{\mathrm{T}}$ of all muons is opposite-sign pairs containing at least one muon with a $\mathrm{p}_{\mathrm{T}}$ greater than $11.0 \mathrm{GeV} / \mathrm{c}$. This spectrum should be multiplied by the charge mismeasurement rate, if it can be obtained from data. To determine the mismeasurement rate, opposite-sign pairs of muons are used. One muon is first chosen randomly. For this muon, the momentum measured in the muon system is used. The invariant mass of the pair is then formed. If the invariant mass 


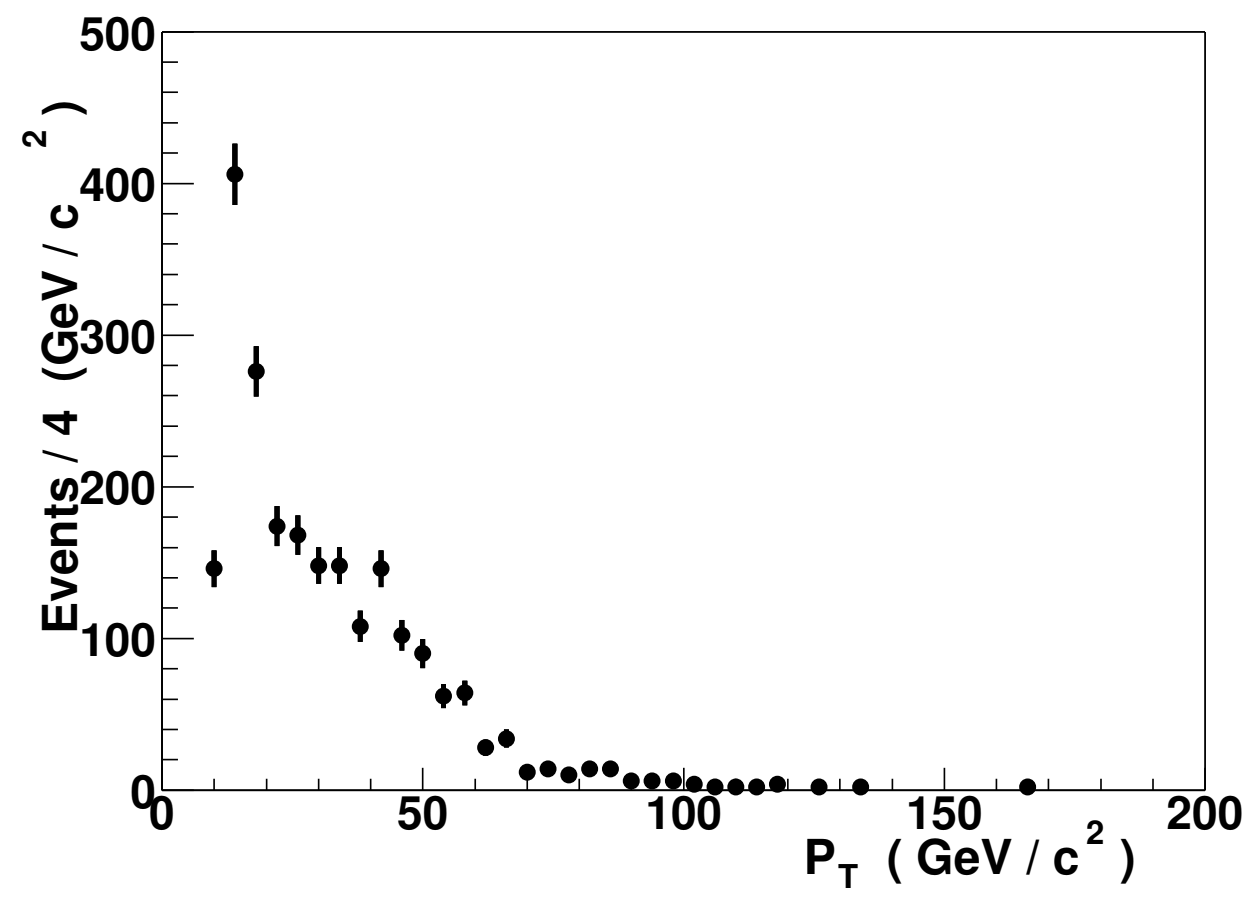

Figure 4.12: The $\mathrm{p}_{\mathrm{T}}$ spectrum of opposite-sign muon pairs as measured in data. These muons could be used to estimate the pool of candidates for sign mismeasurement.

is in the $\mathrm{Z}$ peak, the sign measured by the muon system is compared to the sign measured by the tracking system. The rate of sign mismeasurement is extracted as a function of $\mathrm{p}_{\mathrm{T}}$.

Unfortunately, the invariant mass distribution did not show a clear Z peak. This is likely due to the poor momentum resolution of the muon system at high $\mathrm{p}_{\mathrm{T}}$. This cross-check method is therefore not reliable, and thus was not used.

\subsection{Signal Monte Carlo}

Signal Monte Carlo samples are generated for several sets of values of the input parameters described in Section 1.5. The points in mSUGRA parameter space for which Monte Carlo samples were generated are summarized in Table 4.2. The cross sections generated by PYTHIA are based on leading order calculations in perturbation theory, and have been multiplied by a k-factor of 1.25 to correct them based on other 


\begin{tabular}{cccccccccccc}
\hline SUSY & \multicolumn{10}{c}{$\sigma \times B R$} \\
Point & $m_{0}$ & $m_{1 / 2}$ & $\tan \beta$ & $A_{0}$ & $(\mathrm{pb})$ & $\mathrm{N}_{\exp }$ & $\mathrm{E}$ & $\tilde{\chi}_{1}^{0}$ & $\tilde{\chi}_{2}^{0}$ & $\tilde{\chi}_{1}^{ \pm}$ & $\tilde{l}$ \\
\hline 1 & 68 & 182 & 3 & 0 & .59 & .45 & .0031 & 63 & 115 & 112 & 104 \\
2 & 90 & 180 & 3 & 0 & .20 & .41 & .0085 & 62 & 114 & 110 & 119 \\
3 & 85 & 190 & 5 & 0 & .40 & .20 & .0020 & 68 & 123 & 120 & 118 \\
4 & 108 & 220 & 5 & 0 & .15 & .10 & .0027 & 82 & 147 & 145 & 142 \\
5 & 65 & 170 & 5 & 0 & .83 & .28 & .0014 & 59 & 107 & 103 & 100 \\
6 & 70 & 175 & 3 & 300 & .45 & .55 & .0051 & 58 & 107 & 101 & 103 \\
7 & 75 & 180 & 3 & 300 & .38 & .46 & .0050 & 60 & 111 & 105 & 108 \\
8 & 80 & 185 & 3 & 300 & .33 & .45 & .0057 & 63 & 115 & 110 & 112 \\
9 & 85 & 190 & 3 & 300 & .28 & .45 & .0067 & 65 & 119 & 114 & 117 \\
10 & 85 & 195 & 3 & 300 & .24 & .18 & .0031 & 67 & 123 & 118 & 118 \\
11 & 90 & 200 & 3 & 300 & .20 & .31 & .0064 & 70 & 127 & 123 & 123 \\
$\mathbf{1 2}$ & 76 & 170 & 3 & 0 & .32 & .72 & .0094 & 57 & 106 & 101 & 106 \\
$\mathbf{1 3}$ & 80 & 175 & 3 & 0 & .25 & .67 & .0112 & 60 & 110 & 106 & 110 \\
$\mathbf{1 4}$ & 84 & 180 & 3 & 0 & .21 & .53 & .0105 & 62 & 114 & 110 & 114 \\
15 & 65 & 180 & 3 & 300 & .58 & .52 & .0037 & 60 & 111 & 105 & 101 \\
16 & 70 & 185 & 3 & 300 & .48 & .55 & .0047 & 63 & 115 & 110 & 105 \\
17 & 75 & 190 & 3 & 300 & .37 & .39 & .0044 & 65 & 118 & 114 & 110 \\
$\mathbf{1 8}$ & 72 & 165 & 3 & 0 & .39 & .82 & .0087 & 55 & 102 & 97 & 102 \\
19 & 74 & 168 & 3 & 0 & .35 & .79 & .0094 & 56 & 104 & 100 & 104 \\
$\mathbf{2 0}$ & 88 & 185 & 3 & 0 & .18 & .47 & .0109 & 64 & 118 & 114 & 118 \\
\hline
\end{tabular}

Table 4.2: The values for the 20 points in mSUGRA parameter space chosen for study, the cross section times branching ratio to trileptons for each point, the masses of the two neutralinos $\left(\tilde{\chi}_{1}^{0}\right.$ and $\left.\tilde{\chi}_{2}^{0}\right)$, chargino $\left(\tilde{\chi}_{1}^{ \pm}\right)$, and slepton $(\tilde{l})$ for each point, the number of events expected $\left(\mathrm{N}_{\exp }\right)$ for $239 \mathrm{pb}^{-1}$ of data and the efficiency (E) for each point. The mSUGRA parameters are defined in Section 1.5. The sign $\mu$ parameter is always positive. Points in bold are used in setting a limit in Section 4.9.

next-to-leading order calculations [50]. The CTEQ5L parton distribution functions and PYTHIA version 6.202 are used in generating the signal Monte Carlo samples.

The same information is presented graphically in figures 4.13 and 4.14. In Figure 4.13, the points are separated into those with $\tan \beta=5$, those with $\tan \beta=3$ and $A_{0}=0$, and those with $\tan \beta=3$ and $A_{0}=300$. The points which are used in setting a cross section limit in Section 4.9 are marked in Figure 4.13.

These points are chosen for a few reasons. They all have chargino masses near and above the mass limit set by the LEP experiment. 


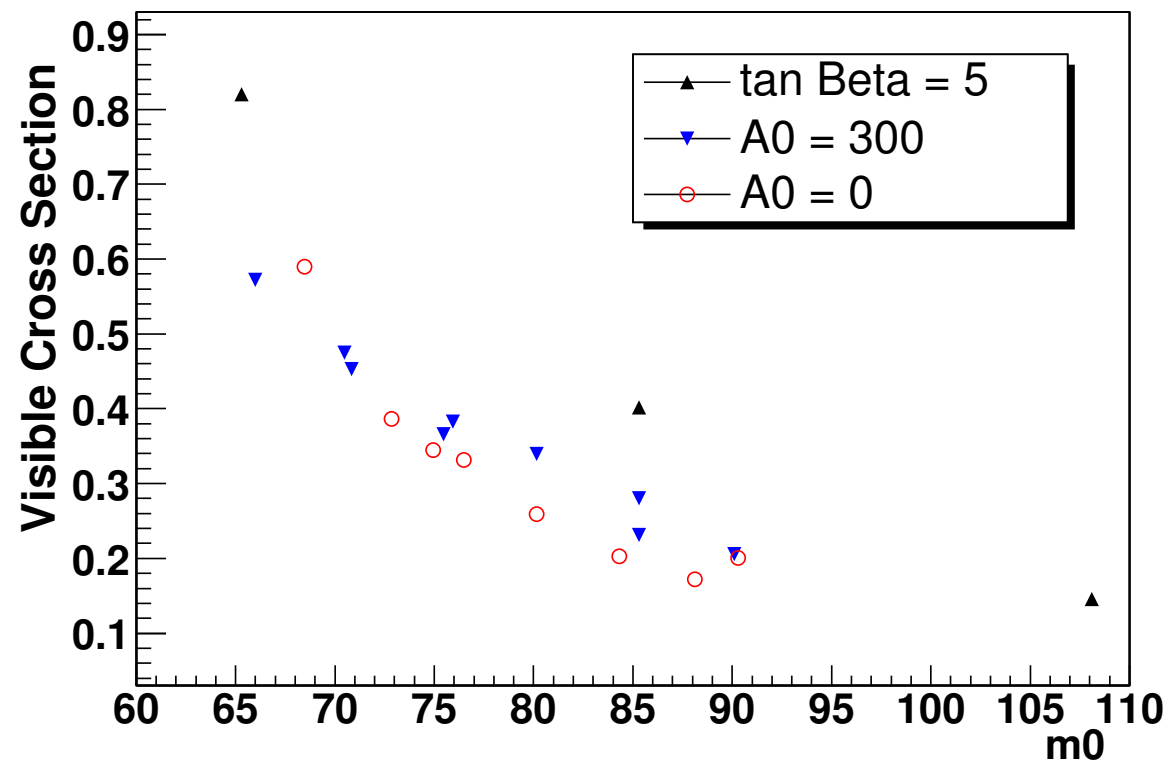

Figure 4.13: The cross section times branching ratio into three leptons as a function of the $m_{0}$ parameter.

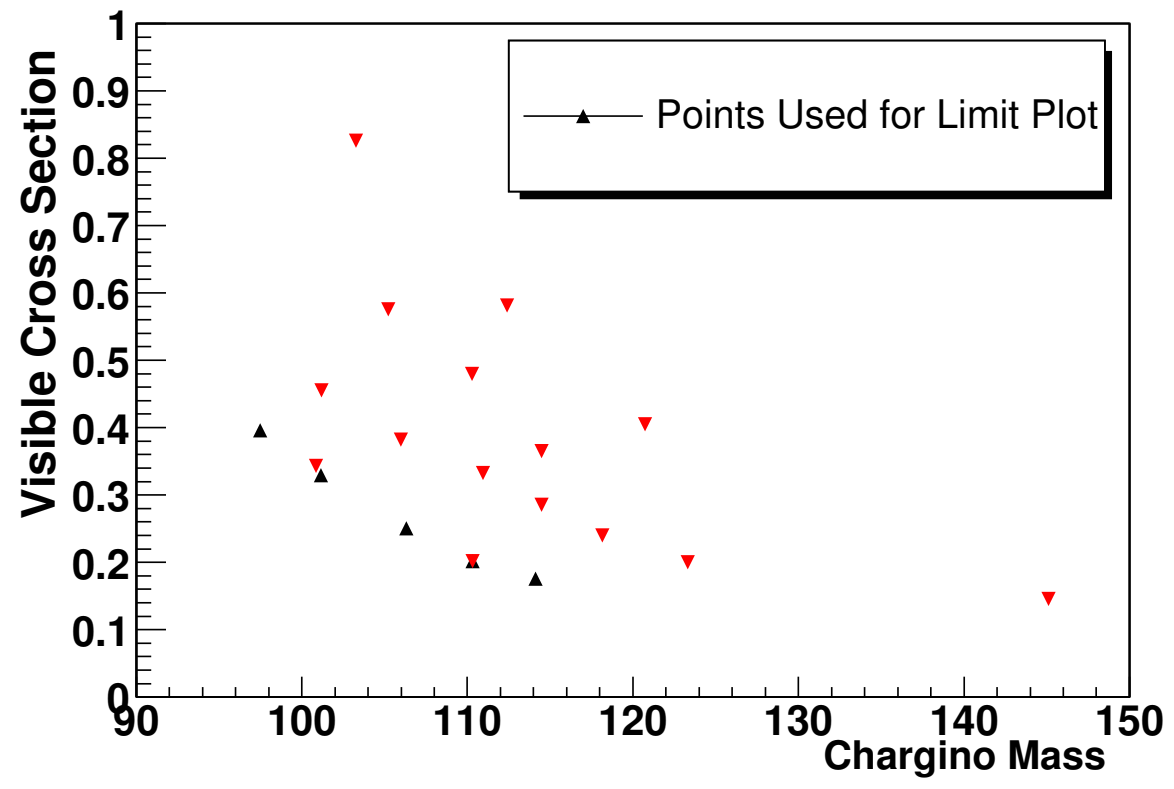

Figure 4.14: The cross section times branching ratio into three leptons as a function of the mass of the chargino.

For this study, a small number of points is chosen, with the knowledge that a more complete scan of the SUSY parameter space will follow. The choice of points is based 
on the masses of the chargino, neutralino, and slepton. If the slepton is lighter than the chargino and neutralino, the chargino and neutralino will decay into the slepton. In this case, the event topology is very dependent on the neutralino-chargino mass difference. The study of this case is postponed due to this complexity.

The case where the slepton is heavier than the neutralino and chargino is chosen. Since the chargino and neutralino decay via virtual gauge bosons, the leptons tend to have larger momenta. This leads to higher efficiencies for these initial studies.

The sign of $\mu$ is taken as positive because this is favored by measurements made by the g-2 experiment [51]. Further studies will include points with the sign of $\mu$ taken as negative.

The points studied here are used to guide the development of analysis cuts. They can be fine-tuned on the fuller scan of SUSY space that will follow.

\subsection{Analysis Cuts}

The final analysis cuts are determined by studying the background and signal Monte Carlo samples. The goal is to minimize the background efficiency while maximizing the signal efficiency.

As a starting point, events must have two loose, $n s e g=3$ (track-matched), isolated, like-sign muons with $\mathrm{p}_{\mathrm{T}}>5 \mathrm{GeV} / \mathrm{c}$. This defines the initial data set, part of which is used to predict the background as described in Section 4.4.1. The $\mathrm{p}_{\mathrm{T}}$ distributions of muons in the b $\bar{b}$ background sample and in a signal Monte Carlo sample (SUSY point 14) are shown in Figure 4.15.

To further reduce the $\mathrm{b} \overline{\mathrm{b}}$ background, a cut is made on the distance in $\phi$ between the two muons. Muons from $b \bar{b}$ decays tend to be back-to-back in $\phi$, as shown in Figure 4.8. Figure 4.16 shows the $\Delta \phi(\mu, \mu)$ distribution for the $b \bar{b}$ background and for a signal Monte Carlo sample (SUSY point 14) In addition, the leading muon $\mathrm{p}_{\mathrm{T}}$ 


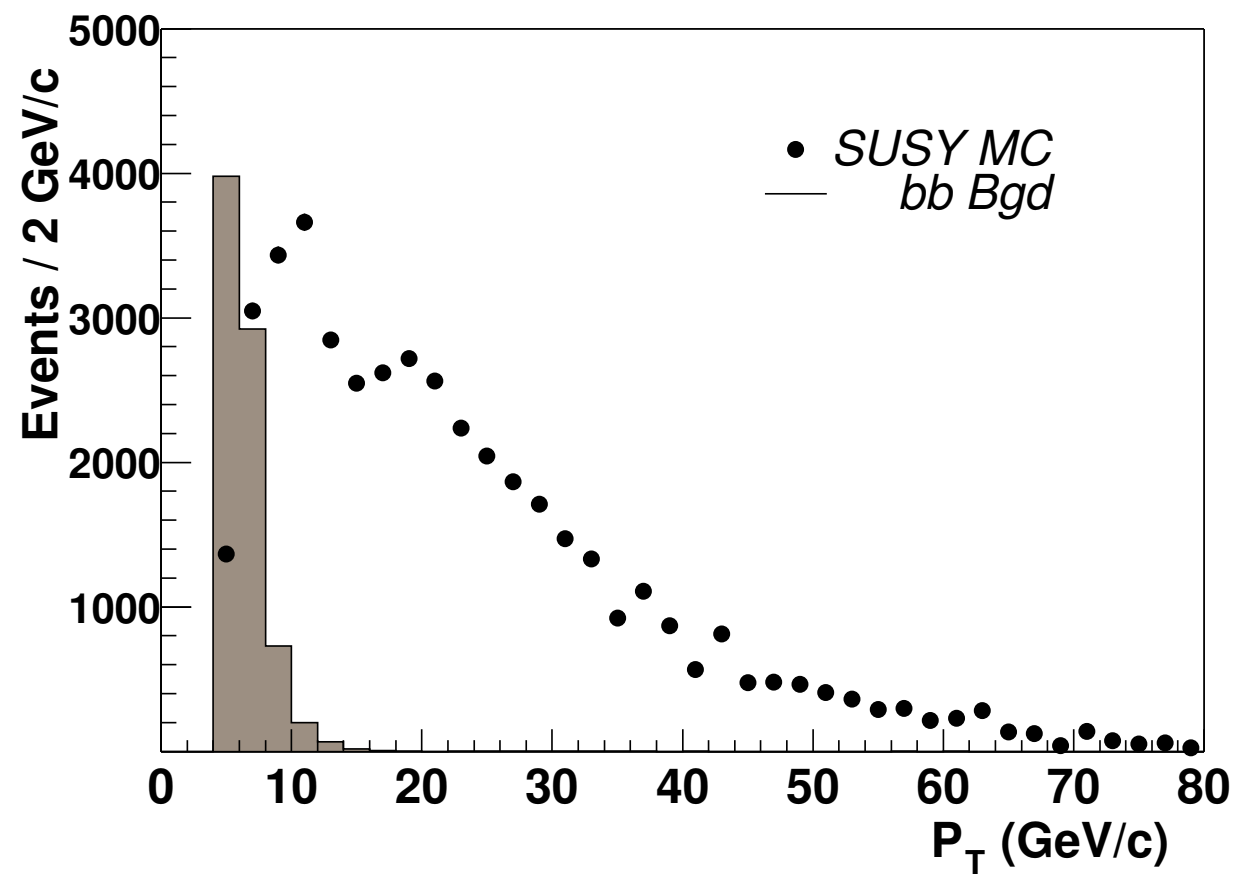

Figure 4.15: The $\mathrm{p}_{\mathrm{T}}$ distribution of muons in the $\mathrm{b} \overline{\mathrm{b}}$ background sample and in a signal Monte Carlo sample (SUSY point 14).

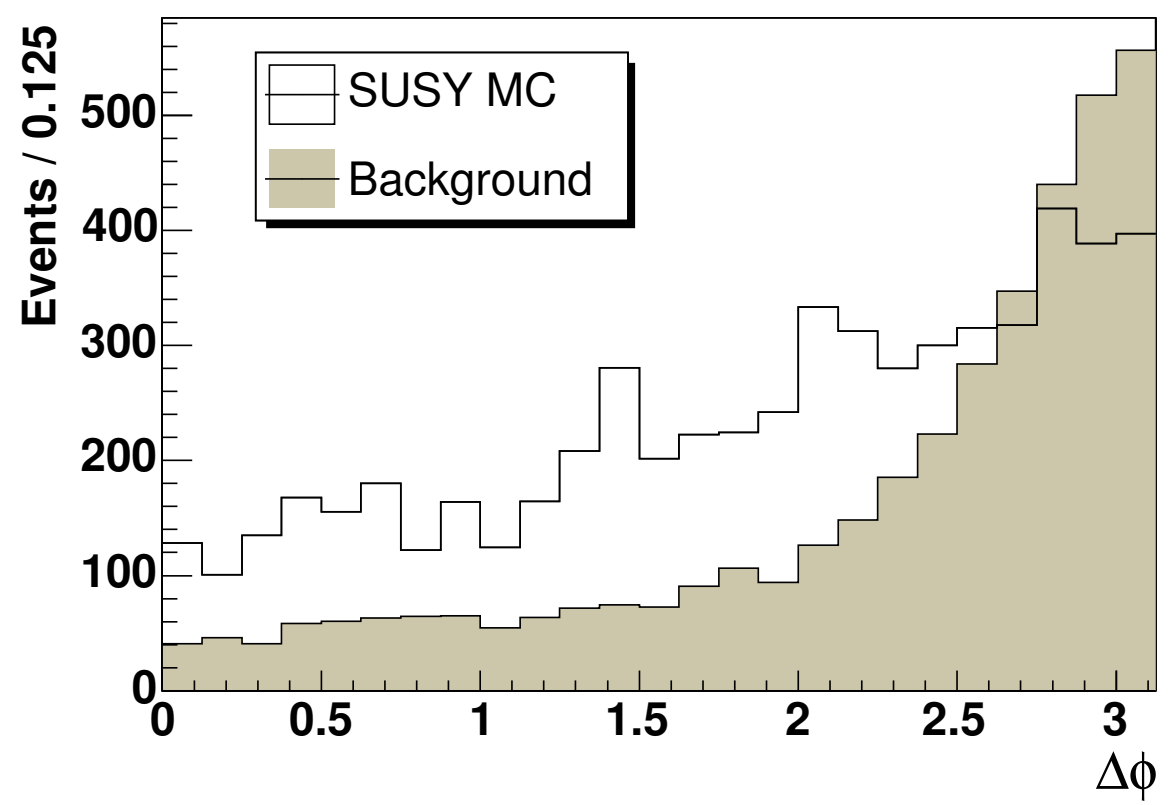

Figure 4.16: The $\Delta \phi(\mu, \mu)$ distribution in the $\mathrm{b} \overline{\mathrm{b}}$ background sample and in a signal Monte Carlo sample (SUSY point 14). 


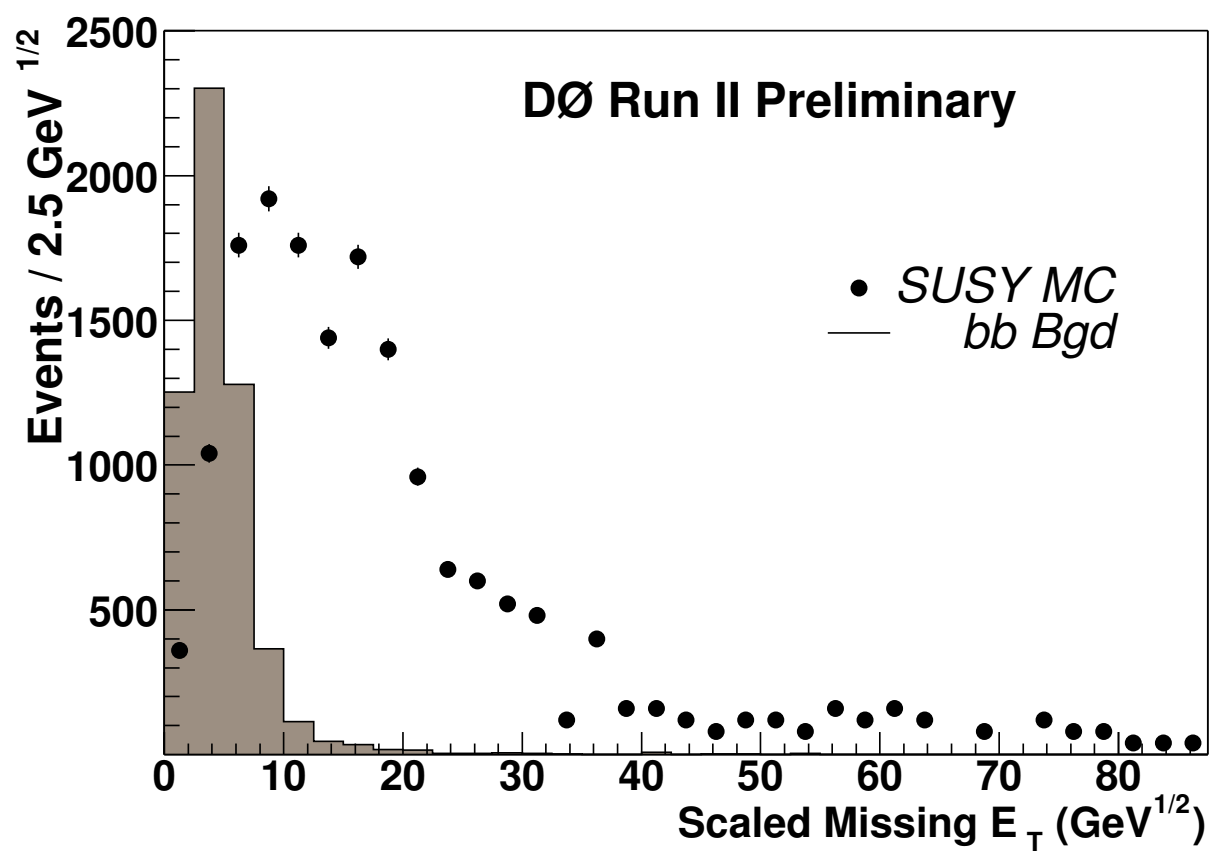

Figure 4.17: The scaled missing transverse energy distribution in the b $\bar{b}$ background sample and in a signal Monte Carlo sample (SUSY point 14).

must be greater than $11 \mathrm{GeV} / \mathrm{c}$. This greatly reduces the background from these low-momentum muons.

Mismeasurements in the calorimeter can lead to artificial MET. To remove this background, a variable called the scaled MET has been developed [52]. It is computed as the MET divided by an estimate of the jet resolution projected onto the direction of the MET. This variable can be used to measure the significance of the MET and to remove events with large MET due to the mismeasurement of the energy of a jet. This variable is defined as:

$$
\text { scaled MET }=\frac{\text { MET }}{\sqrt{\Sigma\left(\sqrt{E_{\text {jet }}} \times \sin \left(\theta_{\text {jet }}\right) \times \mid \cos (\Delta \phi(\text { jet, MET })) \mid\right)^{2}}}
$$

The distributions of scaled MET in the $\mathrm{b} \overline{\mathrm{b}}$ background sample and in a signal Monte Carlo sample (SUSY point 14) are shown in Figure 4.17. A requirement that the 


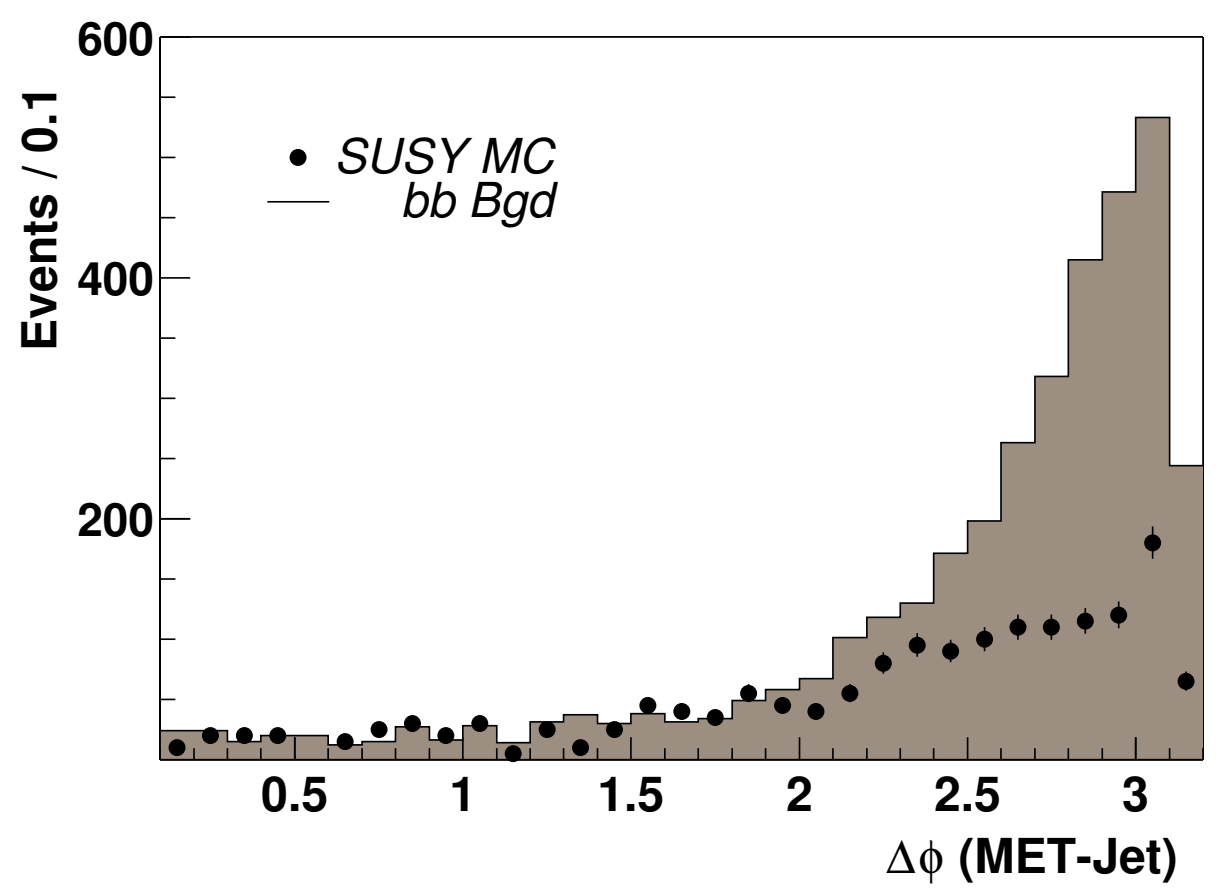

Figure 4.18: The distance in $\phi(\Delta \phi(\mathrm{MET}, \mathrm{Jet}))$ between the direction of the missing transverse energy (MET) in the event and the jet furthest away from the direction of the MET in the $b \bar{b}$ background sample and in a signal Monte Carlo sample (SUSY point 14).

scaled MET must be greater than $8 \mathrm{GeV}^{1 / 2}$ is introduced.

In addition, the separation in $\phi$ between the MET direction and the direction of jets in the event is examined. Events containing b $\bar{b}$ pairs may have jets aligned with the direction of MET from the neutrino produced in the b decay (see Figure 4.18). Also, a jet's energy can be mismeasured, creating artificial MET. A requirement that $(\Delta \phi(J e t, M E T))$ must be less than 2.7 is introduced to remove these events.

Another variable used to discriminate between signal and background is the product of the MET and the $\mathrm{p}_{\mathrm{T}}$ of the lower $\mathrm{p}_{\mathrm{T}}$ muon in the event. It effectively raises the MET requirement when one muon has a low momentum, when there is more background from $b \bar{b}$ production. The distributions of this variable in the $b \bar{b}$ background sample and in a signal Monte Carlo sample (SUSY point 14) are shown in Figure 4.19. A requirement that the product of the MET and the $\mathrm{p}_{\mathrm{T}}$ of the lower $\mathrm{p}_{\mathrm{T}}$ muon in the 


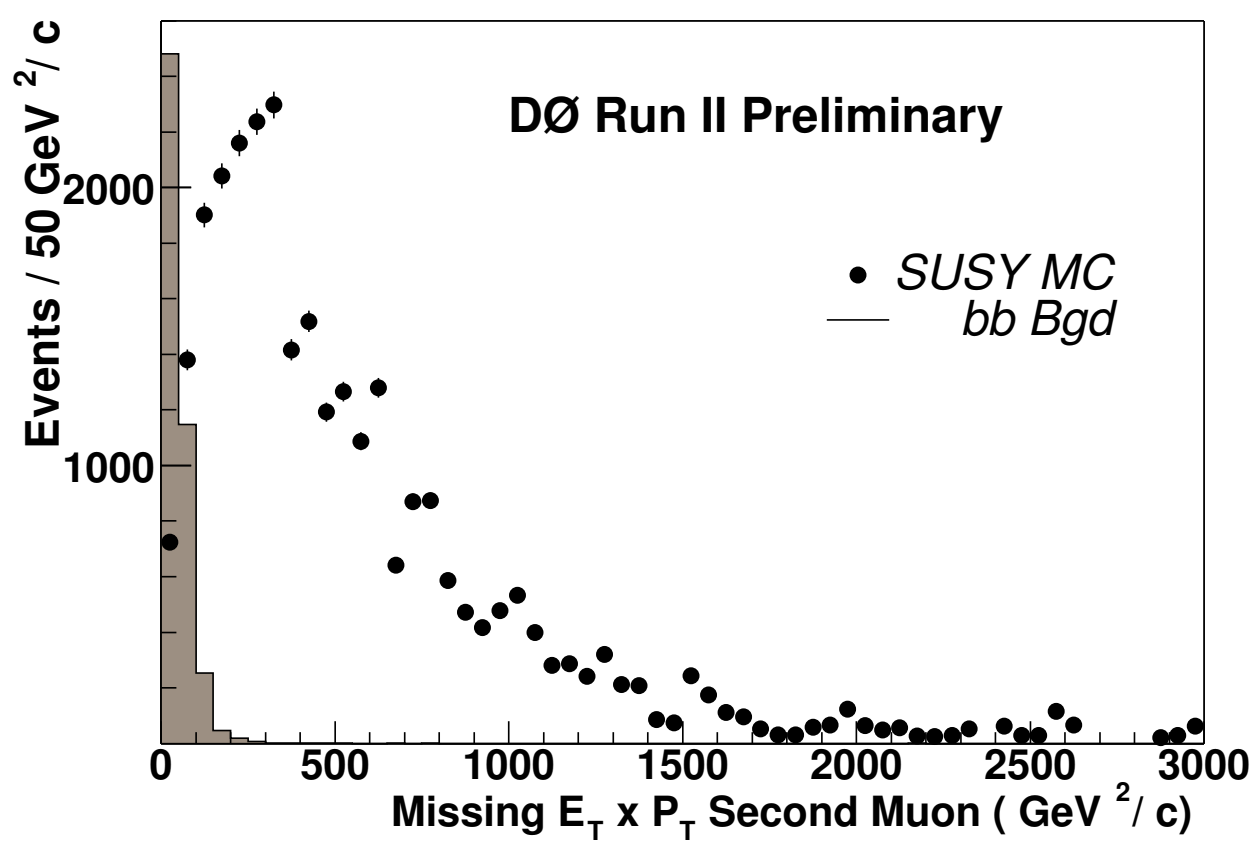

Figure 4.19: The distribution of missing transverse energy multiplied by the $\mathrm{p}_{\mathrm{T}}$ of the lower $\mathrm{p}_{\mathrm{T}}$ muon in the $\mathrm{b} \overline{\mathrm{b}}$ background sample and in a signal Monte Carlo sample (SUSY point 14).

event must be greater than $300 \mathrm{GeV}^{2} / \mathrm{c}$ is introduced. This implies a cut of $60 \mathrm{GeV}$ on the MET when the $\mathrm{p}_{\mathrm{T}}$ of the lower $\mathrm{p}_{\mathrm{T}}$ muon is $5 \mathrm{GeV} / \mathrm{c}$ and a cut of $30 \mathrm{GeV}$ on the MET when the $\mathrm{p}_{\mathrm{T}}$ of the lower $\mathrm{p}_{\mathrm{T}}$ muon is $10 \mathrm{GeV} / \mathrm{c}$.

To remove background events containing $\mathrm{Z}$ bosons, coming from $\mathrm{WZ}, \mathrm{ZZ}$ and $\mathrm{Z} / \gamma$ production, a requirement that the invariant mass of opposite-sign muon pairs must be less than $70 \mathrm{GeV} / \mathrm{c}^{2}$ or greater than $110 \mathrm{GeV} / \mathrm{c}^{2}$ is placed on opposite-sign muon pairs in trimuon events.

An invariant mass cut can also be used to reject background events containing a $\mathrm{Z}$ boson where one muon's charge was misidentified. In this case, the momentum of the misidentified muon tends to be very high, leading to a large invariant mass. A requirement that the mass of like-sign muon pairs must be less than $80 \mathrm{GeV} / \mathrm{c}^{2}$ is introduced.

Cuts to remove muons from cosmic rays were applied as suggested by the $\mathrm{D} \emptyset$ muon 
identification group (see Section 4.1.1). Further studies indicated that the $\Delta \phi(\mu, \mu)<$ 2.7 cut actually removes most of these muons, but no additional significant loss is added by using the muon id cuts, and they remove the few remaining muons from cosmic rays.

In addition to comparing signal and background samples to determine each cut to be used, a permutation study was done to check the relative effect of each cut and to attempt to increase the overall signal efficiency. The permutation study was done using signal Monte Carlo samples. The number of events remaining after all cuts was determined and then checked against the number of events remaining after removing one of the cuts. This was repeated for each cut to check for inefficiencies. The inefficient cuts were then re-examined. This was useful in focusing attention on inefficient cuts in need of further study, as well as in uncovering technical mistakes that were inefficient due to coding errors.

\subsubsection{Summary of Analysis Cuts}

The final analysis cuts are summarized here for clarity:

- Events must have two loose, $n s e g=3$ (track-matched), like-sign muons.

- Both muons must be isolated.

- Both muons must have $\mathrm{p}_{\mathrm{T}}$ greater than $5 \mathrm{GeV} / \mathrm{c}$.

- The distance in $\phi$ between the two muons must be less than 2.7.

- The leading muon $\mathrm{p}_{\mathrm{T}}$ must be greater than $11 \mathrm{GeV} / \mathrm{c}$.

- The product of the MET and the $\mathrm{p}_{\mathrm{T}}$ of the lower $\mathrm{p}_{\mathrm{T}}$ muon in the event must be greater than $300 \mathrm{GeV}^{2} / \mathrm{c}$.

- The scaled MET must be greater than $8 \mathrm{GeV}^{1 / 2}$. 
- If there is a third muon in the event, the invariant mass of opposite-sign muon pairs must be less than $70 \mathrm{GeV} / \mathrm{c}$ or greater than $110 \mathrm{GeV} / \mathrm{c}$.

- The invariant mass of like-sign muon pairs must be less than $80 \mathrm{GeV} / \mathrm{c}$.

- The distance in $\phi$ between the MET direction and the direction of any jet in the event $(\Delta \phi(J e t, M E T))$ must be less than 2.7 .

The effect of these cuts on the signal Monte Carlo samples is shown in Tables 4.3 and 4.4. It is clear from these tables that the sensitivity to this new physics varies depending on the values of the unknown parameters in the theory. This occurs because the masses of the supersymmetric particles and cross sections vary as these values are changed.

\subsection{Comparison of Expected Background to Data}

The effect of the analysis cuts on the background and data samples is shown in Tables 4.5 and 4.6. One event passes the cuts in the data and $0.37 \pm 0.16$ events are expected from the background. The largest remaining backgrounds are from $b \bar{b}$ production and WZ boson production.

Some background from $b \bar{b}$ production remains due to its large cross section. Especially at low momentum, it is difficult to remove all muon pairs originating from this background.

The WZ boson production background is difficult to remove because the $\mathrm{W}$ and $\mathrm{Z}$ particles' superpartners are the chargino and neutralino. Therefore, events from this background are similar in nature to the events that are being sought.

There are several sources of uncertainty in the background estimation. The uncertainty in the luminosity measurement of the data sample is estimated at $6.5 \%$. The trigger and reconstruction efficiency measurements have estimated errors of less than 


\begin{tabular}{|c|c|c|c|c|}
\hline Sample & SUSY 1 & SUSY 2 & SUSY 3 & SUSY 4 \\
\hline Initial & $1.50(.14)$ & $1.07(.07)$ & $0.68(.07)$ & $0.30(.03)$ \\
\hline$\Delta \phi(\mu, \mu)$ & $1.21(.13)$ & $0.84(.06)$ & $0.52(.06)$ & $0.24(.03)$ \\
\hline $\mathrm{p}_{\mathrm{T}}>7$ & $1.21(.13)$ & $0.83(.06)$ & $0.51(.06)$ & $0.23(.03)$ \\
\hline $\mathrm{p}_{\mathrm{T}}>9$ & $1.18(.12)$ & $0.82(.06)$ & $0.50(.06)$ & $0.23(.03)$ \\
\hline $\mathrm{p}_{\mathrm{T}}>11$ & $1.11(.12)$ & $0.80(.06)$ & $0.50(.06)$ & $0.22(.03)$ \\
\hline $\mathrm{MET} \times \mathrm{p}_{\mathrm{T}}>100$ & $1.06(.12)$ & $0.73(.06)$ & $0.45(.06)$ & $0.21(.02)$ \\
\hline $\mathrm{MET} \times \mathrm{p}_{\mathrm{T}}>200$ & $0.81(.10)$ & $0.60(.05)$ & $0.35(.05)$ & $0.19(.02)$ \\
\hline $\mathrm{MET} \times \mathrm{p}_{\mathrm{T}}>300$ & $0.58(.09)$ & $0.51(.05)$ & $0.28(.05)$ & $0.15(.02)$ \\
\hline$\Delta \phi($ Jet,MET $)$ & $0.53(.08)$ & $0.49(.05)$ & $0.26(.05)$ & $0.14(.02)$ \\
\hline LS InvMass & $0.50(.08)$ & $0.44(.05)$ & $0.22(.04)$ & $0.11(.02)$ \\
\hline OS InvMass & $0.47(.08)$ & $0.42(.05)$ & $0.21(.04)$ & $0.11(.02)$ \\
\hline scaled MET > 8 & $0.45(.08)$ & $0.41(.05)$ & $0.20(.04)$ & $0.10(.02)$ \\
\hline Sample & SUSY 5 & SUSY 6 & SUSY 7 & SUSY 8 \\
\hline Initial & $1.17(.14)$ & $1.29(.11)$ & $1.32(.19)$ & $0.95(.13)$ \\
\hline$\Delta \phi(\mu, \mu)$ & $0.87(.12)$ & $1.02(.10)$ & $0.97(.17)$ & $0.80(.12)$ \\
\hline $\mathrm{p}_{\mathrm{T}}>7$ & $0.86(.12)$ & $1.01(.10)$ & $0.97(.17)$ & $0.80(.12)$ \\
\hline $\mathrm{p}_{\mathrm{T}}>9$ & $0.81(.12)$ & $1.00(.09)$ & $0.96(.17)$ & $0.80(.12)$ \\
\hline $\mathrm{p}_{\mathrm{T}}>11$ & $0.74(.11)$ & $1.00(.09)$ & $0.94(.16)$ & $0.79(.12)$ \\
\hline $\mathrm{MET} \times \mathrm{p}_{\mathrm{T}}>100$ & $0.66(.11)$ & $0.94(.09)$ & $0.91(.16)$ & $0.75(.11)$ \\
\hline $\mathrm{MET} \times \mathrm{p}_{\mathrm{T}}>200$ & $0.46(.09)$ & $0.82(.09)$ & $0.76(.15)$ & $0.71(.11)$ \\
\hline $\mathrm{MET} \times \mathrm{p}_{\mathrm{T}}>300$ & $0.34(.08)$ & $0.70(.08)$ & $0.65(.14)$ & $0.63(.10)$ \\
\hline$\Delta \phi($ Jet,MET $)$ & $0.30(.07)$ & $0.67(.08)$ & $0.61(.13)$ & $0.61(.10)$ \\
\hline LS InvMass & $0.29(.07)$ & $0.59(.07)$ & $0.53(.12)$ & $0.54(.10)$ \\
\hline OS InvMass & $0.29(.07)$ & $0.59(.07)$ & $0.53(.12)$ & $0.54(.10)$ \\
\hline scaled MET $>8$ & $0.28(.07)$ & $0.55(.07)$ & $0.46(.12)$ & $0.45(.09)$ \\
\hline Sample & SUSY 9 & SUSY 10 & SUSY 11 & $\begin{array}{l}\text { SUSY } 12 \\
\end{array}$ \\
\hline Initial & $0.94(.13)$ & $0.89(.16)$ & $0.77(.14)$ & $2.05(.09)$ \\
\hline$\Delta \phi(\mu, \mu)$ & $0.79(.12)$ & $0.65(.13)$ & $0.58(.12)$ & $1.61(.08)$ \\
\hline $\mathrm{p}_{\mathrm{T}}>7$ & $0.79(.12)$ & $0.63(.13)$ & $0.58(.12)$ & $1.61(.08)$ \\
\hline $\mathrm{p}_{\mathrm{T}}>9$ & $0.78(.11)$ & 0.63 & $0.55(.12)$ & $1.58(.08)$ \\
\hline $\mathrm{p}_{\mathrm{T}}>11$ & $0.78(.11)$ & $0.61(.13)$ & $0.55(.12)$ & $1.54(.08)$ \\
\hline $\mathrm{MET} \times \mathrm{p}_{\mathrm{T}}>100$ & $0.76(.11)$ & $0.55(.12)$ & $0.51(.12)$ & $1.46(.07)$ \\
\hline $\mathrm{MET} \times \mathrm{p}_{\mathrm{T}}>200$ & $0.70(.11)$ & $0.45(.11)$ & $0.45(.11)$ & $1.23(.07)$ \\
\hline $\mathrm{MET} \times \mathrm{p}_{\mathrm{T}}>300$ & $0.66(.11)$ & $0.33(.10)$ & $0.41(.10)$ & $1.00(.06)$ \\
\hline$\Delta \phi($ Jet,MET $)$ & $0.61(.10)$ & $0.29(.09)$ & $0.39(.10)$ & $0.94(.06)$ \\
\hline LS InvMass & $0.49(.09)$ & $0.23(.08)$ & $0.35(.10)$ & $0.84(.06)$ \\
\hline OS InvMass & $0.49(.09)$ & $0.23(.08)$ & $0.33(.09)$ & $0.82(.06)$ \\
\hline scaled MET $>8$ & $0.45(.09)$ & $0.18(.07)$ & $0.31(.09)$ & $0.72(.05)$ \\
\hline
\end{tabular}

Table 4.3: Number of events after applying each cut to signal Monte Carlo samples for points 1-12. Total errors (sum of statistical and systematic) are in parentheses. 


\begin{tabular}{|c|cccc|}
\hline Sample & SUSY 13 & SUSY 14 & SUSY 15 & SUSY 16 \\
\hline Initial & $1.76(.08)$ & $1.41(.06)$ & $1.75(.17)$ & $1.73(.15)$ \\
$\Delta \phi(\mu, \mu)$ & $1.41(.07)$ & $1.08(.05)$ & $1.45(.15)$ & $1.34(.14)$ \\
$\mathrm{p}_{\mathrm{T}}>7$ & $1.40(.07)$ & $1.08(.05)$ & $1.42(.15)$ & $1.32(.14)$ \\
$\mathrm{p}_{\mathrm{T}}>9$ & $1.38(.07)$ & $1.06(.05)$ & $1.35(.15)$ & $1.29(.13)$ \\
$\mathrm{p}_{\mathrm{T}}>11$ & $1.35(.07)$ & $1.03(.05)$ & $1.29(.14)$ & $1.23(.13)$ \\
MET $\times \mathrm{p}_{\mathrm{T}}>100$ & $1.25(.07)$ & $0.98(.05)$ & $1.20(.14)$ & $1.16(.13)$ \\
$\mathrm{MET} \times \mathrm{p}_{\mathrm{T}}>200$ & $1.08(.06)$ & $0.85(.05)$ & $1.20(.12)$ & $0.94(.11)$ \\
MET $\times \mathrm{p}_{\mathrm{T}}>300$ & $0.88(.06)$ & $0.70(.04)$ & $0.71(.11)$ & $0.74(.10)$ \\
$\Delta \phi(\mathrm{Jet}, \mathrm{MET})$ & $0.84(.05)$ & $0.66(.04)$ & $0.65(.10)$ & $0.71(.10)$ \\
LS InvMass & $0.76(.05)$ & $0.59(.04)$ & $0.60(.10)$ & $0.65(.09)$ \\
OS InvMass & $0.73(.05)$ & $0.56(.04)$ & $0.57(.10)$ & $0.63(.09)$ \\
scaled MET $>8$ & $0.67(.05)$ & $0.53(.04)$ & $0.52(.09)$ & $0.55(.09)$ \\
\hline \hline Sample & SUSY 17 & SUSY 18 & SUSY 19 & SUSY 20 \\
\hline Initial & $1.33(.11)$ & $2.60(.14)$ & $2.21(.12)$ & $1.24(.06)$ \\
$\Delta \phi(\mu, \mu)$ & $0.94(.10)$ & $2.01(.12)$ & $1.76(.11)$ & $0.96(.05)$ \\
$\mathrm{p}_{\mathrm{T}}>7$ & $0.92(.10)$ & $2.00(.12)$ & $1.75(.11)$ & $0.96(.05)$ \\
$\mathrm{p}_{\mathrm{T}}>9$ & $0.89(.09)$ & $1.93(.12)$ & $1.72(.11)$ & $0.95(.05)$ \\
$\mathrm{p}_{\mathrm{T}}>11$ & $0.85(.09)$ & $1.84(.12)$ & $1.62(.10)$ & $0.92(.05)$ \\
MET $\times \mathrm{p}_{\mathrm{T}}>100$ & $0.80(.09)$ & $1.74(.11)$ & $1.51(.10)$ & $0.88(.05)$ \\
$\mathrm{MET} \times \mathrm{p}_{\mathrm{T}}>200$ & $0.68(.08)$ & $1.49(.10)$ & $1.26(.09)$ & $0.77(.05)$ \\
MET $\times \mathrm{p}_{\mathrm{T}}>300$ & $0.55(.07)$ & $1.10(.09)$ & $1.01(.08)$ & $0.65(.04)$ \\
$\Delta \phi(\mathrm{Jet}, \mathrm{MET})$ & $0.52(.07)$ & $1.03(.09)$ & $0.95(.08)$ & $0.61(.04)$ \\
LS InvMass & $0.45(.07)$ & $0.95(.08)$ & $0.87(.08)$ & $0.56(.04)$ \\
OS InvMass & $0.43(.07)$ & $0.93(.08)$ & $0.84(.07)$ & $0.53(.04)$ \\
scaled MET $>8$ & $0.39(.06)$ & $0.82(.08)$ & $0.79(.07)$ & $0.47(.04)$ \\
\hline
\end{tabular}

Table 4.4: Number of events after applying each cut to signal Monte Carlo samples for points 13-20. Total errors (sum of statistical and systematic) are in parentheses. 


\begin{tabular}{|c|c|c|c|c|}
\hline Sample & $\overline{\mathrm{b} \overline{\mathrm{b}}}$ & WZ & $\mathrm{ZZ}$ & $\overline{t \bar{t}}$ \\
\hline Initial & $3961.05(41.24)$ & $0.53(.17)$ & $0.08(.05)$ & $0.01(.01)$ \\
\hline$\Delta \phi(\mu, \mu)$ & $2226.71(31.03)$ & $0.41(.14)$ & $0.06(.04)$ & $0.01(.01)$ \\
\hline $\mathrm{p}_{\mathrm{T}}>7$ & $902.29(18.00)$ & $0.41(.14)$ & $0.06(.04)$ & $0.01(.01)$ \\
\hline $\mathrm{p}_{\mathrm{T}}>9$ & $269.60(8.73)$ & $0.41(.14)$ & $0.06(.04)$ & $0.01(.01)$ \\
\hline $\mathrm{p}_{\mathrm{T}}>11$ & $91.85(4.68)$ & $0.41(.14)$ & $0.06(.04)$ & $0.01(.01)$ \\
\hline $\mathrm{MET} \times \mathrm{p}_{\mathrm{T}}>100$ & $19.83(2.15)$ & $0.41(.14)$ & $0.04(.03)$ & $0.01(.01)$ \\
\hline $\mathrm{MET} \times \mathrm{p}_{\mathrm{T}}>200$ & $6.88(1.32)$ & $0.37(.14)$ & $0.04(.03)$ & $0.01(.01)$ \\
\hline $\mathrm{MET} \times \mathrm{p}_{\mathrm{T}}>300$ & $1.42(.58)$ & $0.37(.14)$ & $0.04(.03)$ & $0.01(.01)$ \\
\hline$\Delta \phi($ Jet,MET $)$ & $1.012(.51)$ & $0.37(.14)$ & $0.04(.03)$ & $0.01(.01)$ \\
\hline LS InvMass & $0.61(.35)$ & $0.21(.10)$ & $0.01(.02)$ & $0(0)$ \\
\hline OS InvMass & $0.61(.35)$ & $0.15(.09)$ & $0(0)$ & $0(0)$ \\
\hline scaled MET $>8$ & $0.17(.12)$ & $0.15(.09)$ & $0(0)$ & $0(0)$ \\
\hline Sample & $\overline{Z b b}$ & $\overline{\mathrm{Wj}}$ & $\overline{\mathrm{Wjj}}$ & $\overline{\mathrm{Wbb}}$ \\
\hline Initial & $0.06(.01)$ & $3.50(2.73)$ & 0 & $0.31(.07)$ \\
\hline$\Delta \phi(\mu, \mu)$ & $0.04(.01)$ & $3.50(2.73)$ & 0 & $0.26(.06)$ \\
\hline $\mathrm{p}_{\mathrm{T}}>7$ & $0.04(.01)$ & $3.50(2.73)$ & 0 & $0.25(.06)$ \\
\hline $\mathrm{p}_{\mathrm{T}}>9$ & $0.04(.01)$ & $3.50(2.73)$ & 0 & $0.25(.06)$ \\
\hline $\mathrm{p}_{\mathrm{T}}>11$ & $0.04(.01)$ & $3.50(2.73)$ & 0 & $0.25(.06)$ \\
\hline $\mathrm{MET} \times \mathrm{p}_{\mathrm{T}}>100$ & $0.02(.01)$ & $2.38(2.23)$ & 0 & $0.23(.06)$ \\
\hline $\mathrm{MET} \times \mathrm{p}_{\mathrm{T}}>200$ & $0.02(.01)$ & $2.38(2.23)$ & 0 & $0.15(.05)$ \\
\hline $\mathrm{MET} \times \mathrm{p}_{\mathrm{T}}>300$ & $0.01(.01)$ & $1.08(1.58)$ & 0 & $0.09(.04)$ \\
\hline$\Delta \phi($ Jet,MET $)$ & $0.01(.01)$ & $1.08(1.58)$ & 0 & $0.06(.03)$ \\
\hline LS InvMass & $0.01(.01)$ & $0(0)$ & 0 & $0.06(.03)$ \\
\hline OS InvMass & $0.00(.00)$ & $0(0)$ & 0 & $0.06(.03)$ \\
\hline scaled MET $>8$ & $0.00(.00)$ & $0(0)$ & 0 & $0.05(.03)$ \\
\hline Sample & $\overline{\mathrm{Z} / \gamma(15-60)}$ & 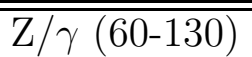 & $\overline{\mathrm{ZZ} / \gamma(130-250)}$ & \\
\hline Initial & $1.41(1.41)$ & $40.59(7.22)$ & $0.52(.12)$ & \\
\hline$\Delta \phi(\mu, \mu)$ & $1.41(1.41)$ & $6.43(2.90)$ & $0.03(.03)$ & \\
\hline $\mathrm{p}_{\mathrm{T}}>7$ & $0(0)$ & $6.43(2.90)$ & $0.03(.03)$ & \\
\hline $\mathrm{p}_{\mathrm{T}}>9$ & $0(0)$ & $6.43(2.90)$ & $0.03(.03)$ & \\
\hline $\mathrm{p}_{\mathrm{T}}>11$ & $0(0)$ & $6.43(2.90)$ & $0.03(.03)$ & \\
\hline $\mathrm{MET} \times \mathrm{p}_{\mathrm{T}}>100$ & $0(0)$ & $5.49(2.73)$ & $0.03(.03)$ & \\
\hline $\mathrm{MET} \times \mathrm{p}_{\mathrm{T}}>200$ & $0(0)$ & $4.36(2.41)$ & $0.03(.03)$ & \\
\hline $\mathrm{MET} \times \mathrm{p}_{\mathrm{T}}>300$ & $0(0)$ & $3.56(2.23)$ & $0.03(.03)$ & \\
\hline$\Delta \phi($ Jet,MET $)$ & $0(0)$ & $3.56(2.23)$ & $0.03(.03)$ & \\
\hline LS InvMass & $0(0)$ & $0(0)$ & $0(0)$ & \\
\hline OS InvMass & $0(0)$ & $0(0)$ & $0(0)$ & \\
\hline scaled MET $>8$ & $0(0)$ & $0(0)$ & $0(0)$ & \\
\hline
\end{tabular}

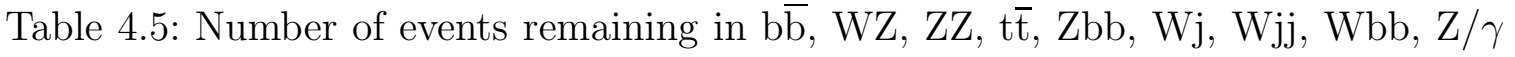
background samples after applying each cut to the samples. Total errors (sum of statistical and systematic) are in parentheses. 


\begin{tabular}{|c|c|c|}
\hline Sample & Background & Data \\
\hline Initial & $4008.06(42.09)$ & 4223 \\
$\Delta \phi(\mu, \mu)$ & $2238.85(31.33)$ & 2417 \\
$\mathrm{p}_{\mathrm{T}}>7$ & $913.01(18.45)$ & 966 \\
$\mathrm{p}_{\mathrm{T}}>9$ & $280.32(9.62)$ & 285 \\
$\mathrm{p}_{\mathrm{T}}>11$ & $102.57(6.19)$ & 95 \\
$\mathrm{MET} \times \mathrm{p}_{\mathrm{T}}>100$ & $28.43(4.37)$ & 26 \\
$\mathrm{MET} \times \mathrm{p}_{\mathrm{T}}>200$ & $14.22(3.74)$ & 9 \\
$\mathrm{MET} \times \mathrm{p}_{\mathrm{T}}>300$ & $3.02(1.71)$ & 6 \\
$\Delta \phi(\mathrm{Jet}, \mathrm{MET})$ & $1.49(0.54)$ & 4 \\
$\mathrm{LS} \mathrm{InvMass}$ & $0.90(0.37)$ & 3 \\
OS InvMass & $0.82(0.37)$ & 2 \\
scaled MET $>8$ & $0.37(0.16)$ & 1 \\
\hline
\end{tabular}

Table 4.6: Number of events remaining in data and the number expected from the sum of all backgrounds after applying each cut. Total errors (sum of statistical and systematic) are in parentheses.

$1 \%$. The systematic errors from the uncertainty on the Monte Carlo cross sections are estimated at $10 \%$. This estimate is made by comparing the cross sections generated by PYTHIA with measured cross sections.

There are also systematic errors arising from differences between the Monte Carlo and data in the $\mathrm{MET} \times \mathrm{p}_{\mathrm{T}}$ and $\Delta \phi(\mathrm{MET}$, Jet) distributions. The differences in these distributions are checked by comparing Z Monte Carlo and data samples. The percentages of events beyond the cut value in Monte Carlo and data samples are measured and the difference is used as an estimate of the systematic error on the background. The errors are estimated at $10 \%$ for $\mathrm{MET} \times \mathrm{p}_{\mathrm{T}}$ and $10 \%$ for $\Delta \phi(\mathrm{MET}$, Jet).

Three event displays of the event in the data passing all of the cuts are shown in Figures $4.20,4.21$, and 4.22 . Table 4.7 shows some relevant information about this event. 


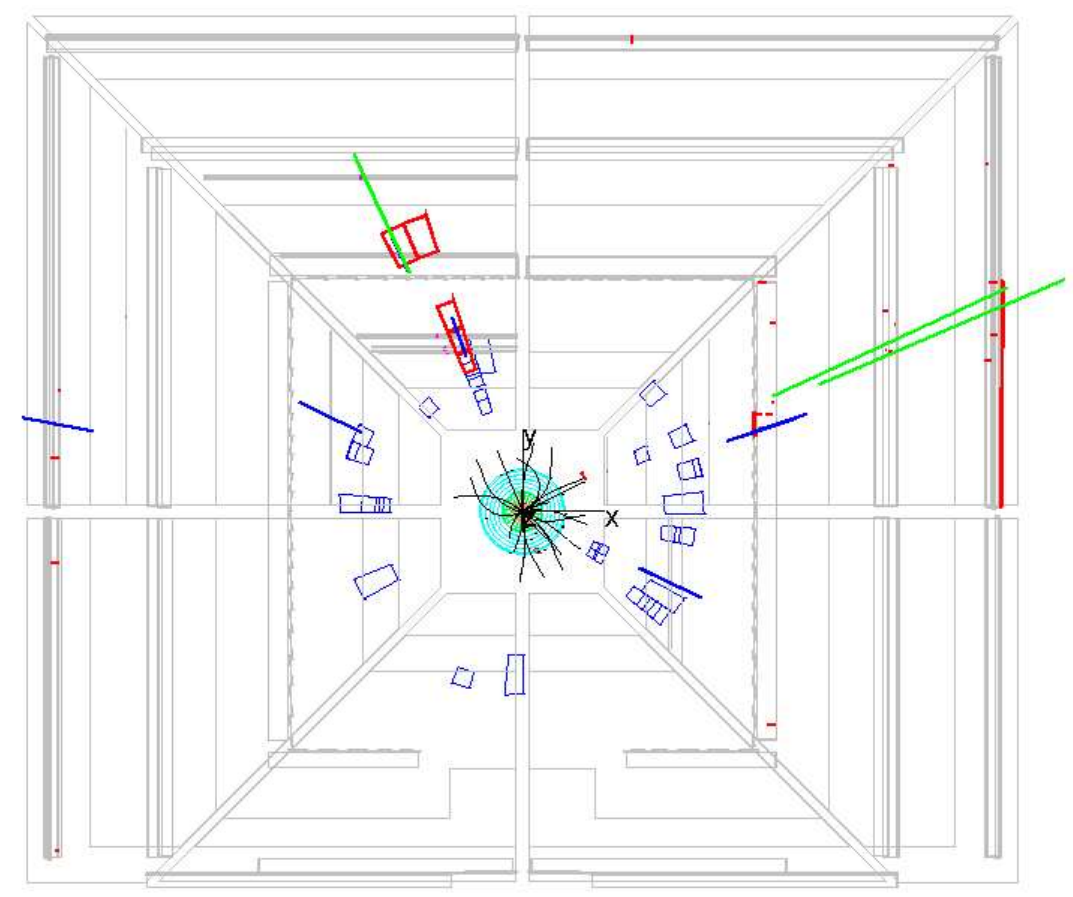

Figure 4.20: Event display of the event passing all cuts in data, event number 44096517 from run 177010. In the middle of the display, reconstructed charged-particle tracks can be seen. The green lines represent muons traversing the detector.

\begin{tabular}{ccccccccc}
\hline Object & $\mathrm{E}$ & $\mathrm{p}_{\mathrm{T}}$ & $\mathrm{p}_{x}$ & $\mathrm{p}_{y}$ & $\mathrm{p}_{z}$ & $\eta$ & $\phi$ & charge \\
\hline Muon 1 & 14.1 & 13.8 & 12.5 & 5.8 & -2.6 & -0.19 & 0.43 & + \\
Muon 2 & 62.2 & 29.1 & -10.9 & 26.9 & -55.0 & -1.39 & 1.96 & + \\
MET & & 33.5 & -13.9 & -30.4 & & & 4.28 & \\
\hline
\end{tabular}

Table 4.7: Table showing the kinematic values for the two muons and MET in run 177010 , event 44096517.

\subsection{Combining Results with Other Channels}

Searches for the associated production of chargino and neutralino sparticles have been conducted using the electron, muon, and track final state, the dielectron and track final state, and the dimuon and track final state [52-54]. Better limits on the cross section for associated production of chargino and neutralino sparticles with leptonic final states are set by combining the results from those searches and the 


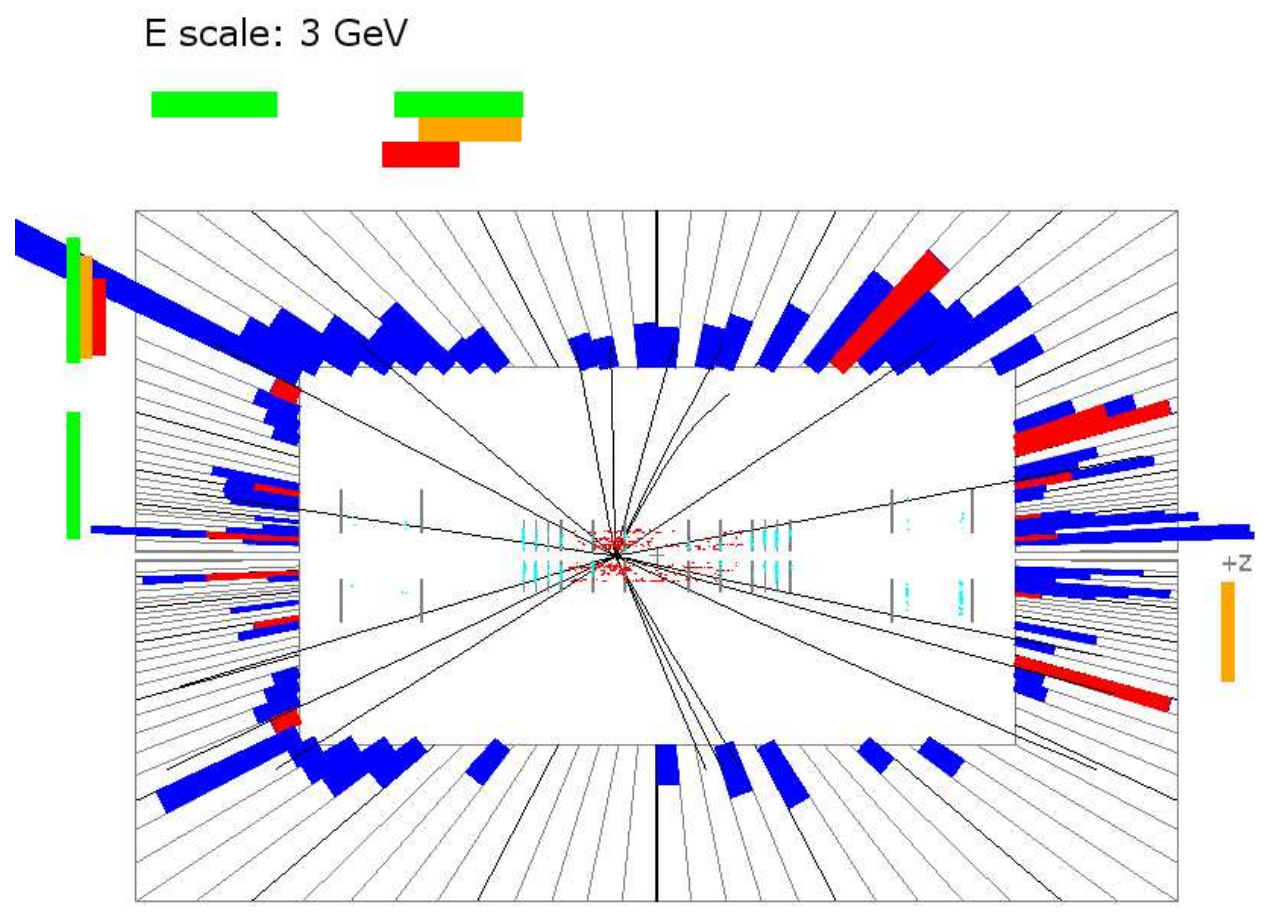

Figure 4.21: Event display of the event passing all cuts in data, event number 44096517 from run 177010. Reconstructed tracks are visible in the center of the display. The red, orange and green bars are hits in the A, B, and C layers of the muon system.

\begin{tabular}{ccccc}
\hline Channel & Luminosity $\left(\mathrm{pb}^{-1}\right)$ & Data & Expected Background & $\mathrm{N}_{\text {excluded }}$ \\
\hline $\mathrm{e}+\mathrm{e}+$ track & 249 & 1 & $0.68 \pm 0.40$ & 5.0 \\
$\mu+\mu+$ track & 221 & 1 & $1.83 \pm 0.40$ & 5.7 \\
$\mathrm{e}+\mu+$ track & 235 & 0 & $0.29 \pm 0.33$ & 3.2 \\
LS $\mu \mu$ & 239 & 1 & $0.37 \pm 0.16$ & 4.4 \\
Combined & & 3 & $3.17 \pm 0.67$ & 4.8 \\
\hline
\end{tabular}

Table 4.8: Luminosity, number of observed candidate events, expected number of background events for each final state used in determining the combined cross section limit, and the excluded number of signal events $\left(\mathrm{N}_{\text {excluded }}\right)$.

search described in this analysis [55].

The relevant data from those channels are summarized in Table 4.8. The combination of results is performed using the LEP CLS method [56]. The number of excluded signal events is given for each channel separately, and is independent of the SUSY models used. 


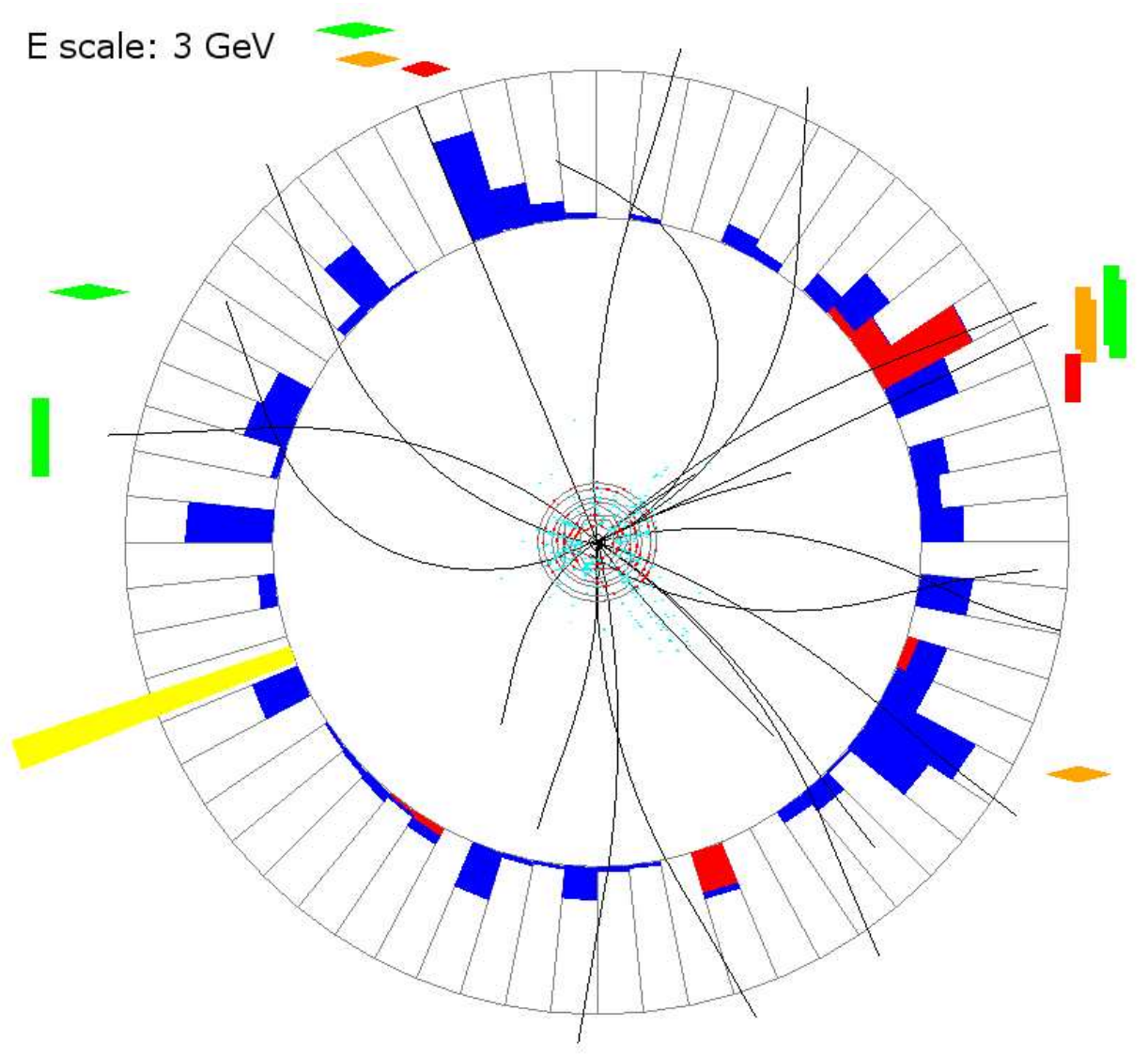

Figure 4.22: Event display of the event passing all cuts in data, event number 44096517 from run 177010. Reconstructed tracks are visible in the center of the display. The red, orange and green bars on the outer part of the display are hits in the A, B, and $\mathrm{C}$ layers of the muon system. The red and blue blocks represent energy in the calorimeter and the yellow block represents missing energy.

\subsubsection{CLS Method}

The LEP CLS method [56] is summarized here for completeness.

The likelihood ratio can be written as

$$
X=\prod_{i=1}^{n} X_{i}
$$

with

$$
X_{i}=\frac{e^{-\left(s_{i}+b_{i}\right)}\left(s_{i}+b_{i}\right)^{d_{i}}}{d_{i} !} / \frac{e^{-b_{i} b_{i}^{d_{i}}}}{d_{i} !} .
$$


where $s_{i}$ is the estimated signal in the $i$ th channel, $b_{i}$ is the estimated background in the $i$ th channel, and $d_{i}$ is the number of observed candidates.

The confidence level for excluding the presence of both signal and background is

$$
C L_{s+b}=P_{s+b}\left(X \leq X_{o b s}\right),
$$

the probability, assuming the presence of signal and background, that the likelihood ratio would be less than or equal to that observed in the data. This probability is

$$
P_{s+b}\left(X \leq X_{o b s}\right)=\sum_{X\left(\left\{d_{i}^{\prime}\right\}\right) \leq X\left(\left\{d_{i}\right\}\right)} \prod_{i=1}^{n} \frac{e^{-\left(s_{i}+b_{i}\right)}\left(s_{i}+b_{i}\right)^{d_{i}^{\prime}}}{d_{i}^{\prime} !}
$$

where $X\left(\left\{d_{i}\right\}\right)$ is the likelihood computed for the observed set of candidates in each channel $\left\{d_{i}\right\}$, summed over all outcomes $\left\{d_{i}^{\prime}\right\}$ with likelihoods less than or equal to the observed one.

The confidence level for the background alone,

$$
C L_{b}=P_{b}\left(X \leq X_{o b s}\right),
$$

can also be computed.

The confidence level $C L_{s}$ is then computed as the ratio

$$
C L_{s}=C L_{s+b} / C L_{b} .
$$

The uncertainties in the signal and background must also be incorporated. $X_{i}$ is modified by averaging over possible values of the signal and background given by their uncertainty probability distributions. These are assumed to be Gaussian, with 
the lower tail cut off at zero. $X_{i}$ is replaced by

$$
X_{i} \rightarrow \frac{\int_{0}^{\infty} d s^{\prime} \int_{0}^{\infty} d b^{\prime} \frac{e^{-\left(\left(s^{\prime}-s_{i}\right)^{2} / 2 \sigma_{s_{i}}^{2}+\left(b^{\prime}-b_{i}\right)^{2} / 2 \sigma_{b_{i}}^{2}\right)}}{2 \pi \sigma s_{i} \sigma_{b_{i}}} X_{i}}{\int_{0}^{\infty} d s^{\prime} \int_{0}^{\infty} d b^{\prime} \frac{e^{-\left(\left(s^{\prime}-s_{i}\right)^{2} / 2 \sigma_{s_{i}}^{2}+\left(b^{\prime}-b_{i}\right)^{2} / 2 \sigma_{b_{i}}^{2}\right)}}{2 \pi \sigma_{s_{i}} \sigma_{b_{i}}}}
$$

To derive a limit on the cross section for a given SUSY point, the cross section corresponding to that point is disregarded, and the cross section is treated as a free parameter. The efficiency and uncertainty for the SUSY point are used. The confidence level is then calculated as a function of the cross section. The value of the cross section that corresponds to a confidence level of $95 \%$ is interpreted as the cross section limit for the SUSY point. This can be repeated at several SUSY points to obtain a cross section limit for each point. Since each point corresponds to a chargino mass, a cross section limit as a function of the chargino mass is obtained.

\subsection{Cross Section Limit}

A 95\% confidence level limit on the total cross section for associated chargino and neutralino production with leptonic final states has been set. The limit is presented as a function of the $\tilde{\chi}_{1}^{ \pm}$mass (see Figure 4.24 and Table 4.9). SUSY points 12, 13, 14, 18, and 20 are used in setting the limit. These points are chosen because they have slepton masses equal to or just above the chargino and neutralino masses. These were chosen to force the decays of the chargino and neutralino via virtual gauge bosons, which results in higher $\mathrm{p}_{\mathrm{T}}$ leptons in the final state.

Chargino masses below $103 \mathrm{GeV} / \mathrm{c}^{2}$ are excluded by direct searches at LEP, while this search nearly excludes masses above this threshold. Masses below $97 \mathrm{GeV} / \mathrm{c}^{2}$ are excluded by the present search. For comparison, the limit in the like-sign dimuon final state alone is shown in Figure 4.23. 


\begin{tabular}{ccccccc}
\hline SUSY & $\sigma \times B R$ & $\mathrm{~N}_{\exp }$ & $\mathrm{E}$ & $\tilde{\chi}_{1}^{ \pm}$ & $\sigma_{\text {lim }}^{\mu \mu}$ & $\sigma_{\text {lim }}^{\text {total }}$ \\
\hline 1 & .59 & .45 & .0031 & 112 & & \\
2 & .20 & .41 & .0085 & 110 & & \\
3 & .40 & .20 & .0020 & 120 & & \\
4 & .15 & .10 & .0027 & 145 & & \\
5 & .83 & .28 & .0014 & 103 & & \\
6 & .45 & .55 & .0051 & 101 & & \\
7 & .38 & .46 & .0050 & 105 & & \\
8 & .33 & .45 & .0057 & 110 & & \\
9 & .28 & .45 & .0067 & 114 & & \\
10 & .24 & .18 & .0031 & 118 & & \\
11 & .20 & .31 & .0064 & 123 & & \\
$\mathbf{1 2}$ & .32 & .72 & .0094 & 101 & 1.98 & 0.38 \\
$\mathbf{1 3}$ & .25 & .67 & .0112 & 106 & 1.79 & 0.35 \\
$\mathbf{1 4}$ & .21 & .53 & .0105 & 110 & 1.76 & 0.33 \\
15 & .58 & .52 & .0037 & 105 & & \\
16 & .48 & .55 & .0047 & 110 & & \\
17 & .37 & .39 & .0044 & 114 & & \\
$\mathbf{1 8}$ & .39 & .82 & .0087 & 97 & 2.11 & 0.40 \\
19 & .35 & .79 & .0094 & 100 & & \\
$\mathbf{2 0}$ & .18 & .47 & .0109 & 114 & 1.70 & 0.30 \\
\hline
\end{tabular}

Table 4.9: For the SUSY points, the cross section times branching ratio to trileptons, the mass of the chargino $\left(\tilde{\chi}_{1}^{ \pm}\right)$, the number of events expected $\left(\mathrm{N}_{\exp }\right)$ and the efficiency (E) for each point for $239 \mathrm{pb}^{-1}$ of data, and the cross section limit in the like-sign dimuon channel $\left(\sigma_{l i m}^{\mu \mu}\right)$ and overall $\left(\sigma_{\text {lim }}^{\text {total }}\right)$. Points in bold are used in Figures 4.24 and 4.23 .

This result improves on the $\mathrm{D} \varnothing$ and CDF Run I results [57,58] for the regions of SUSY space it explores. For the D $\varnothing$ Run I result, searches were done in the eee, ee $\mu$, $\mathrm{e} \mu \mu$, and $\mu \mu \mu$ final states with between $75 \mathrm{pb}^{-1}$ and $95 \mathrm{pb}^{-1}$ of data, and limits were set at the $95 \%$ confidence level. Data currently being collected will permit further improvement, including the capability to exclude $\tilde{\chi}_{1}^{ \pm}$masses above the LEP limit. 


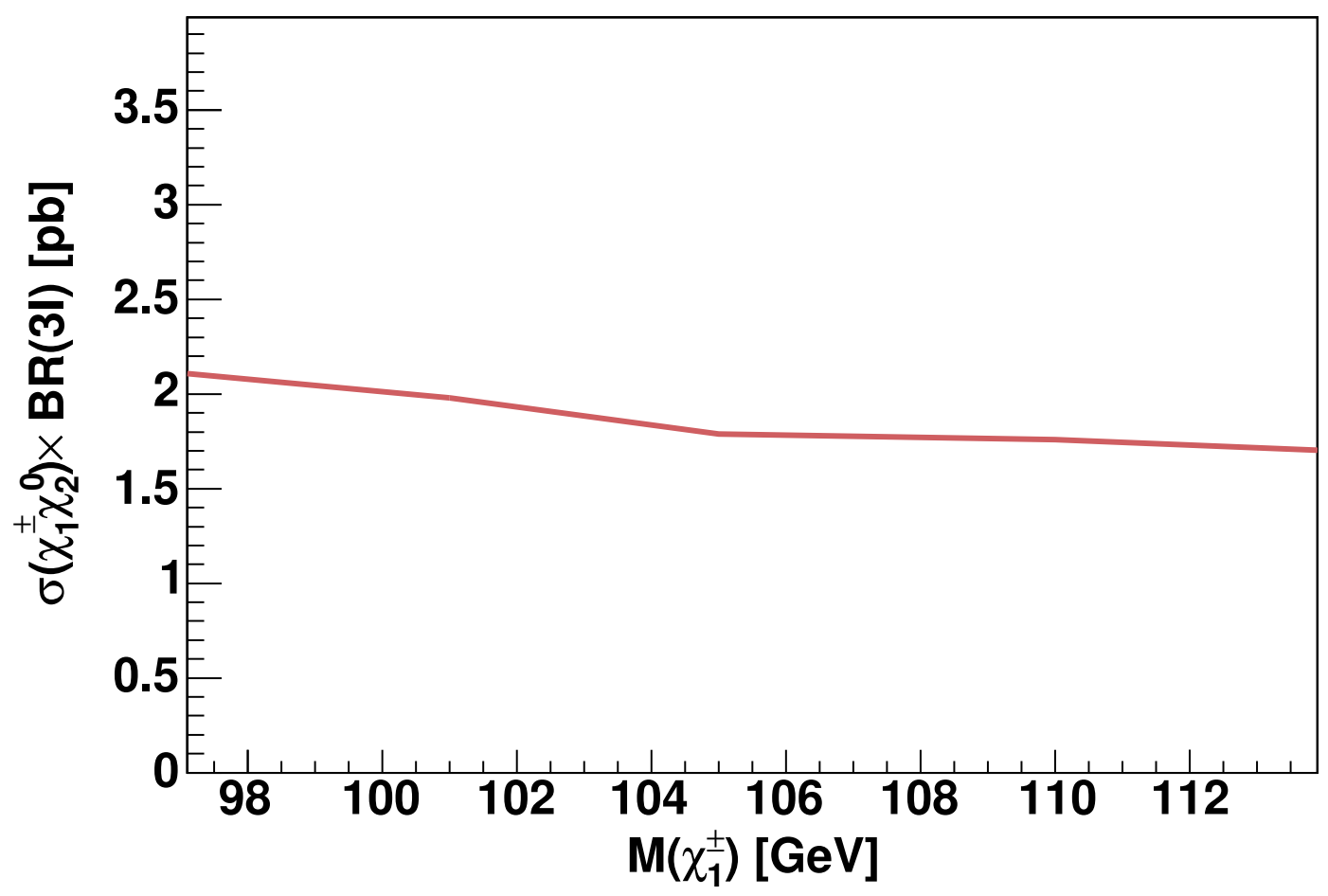

Figure 4.23: Limit on the total cross section for associated chargino $\left(\tilde{\chi}_{1}^{ \pm}\right)$and neutralino $\left(\tilde{\chi}_{2}^{0}\right)$ production in the like-sign dimuon channel as a function of the $\tilde{\chi}_{1}^{ \pm}$mass. 


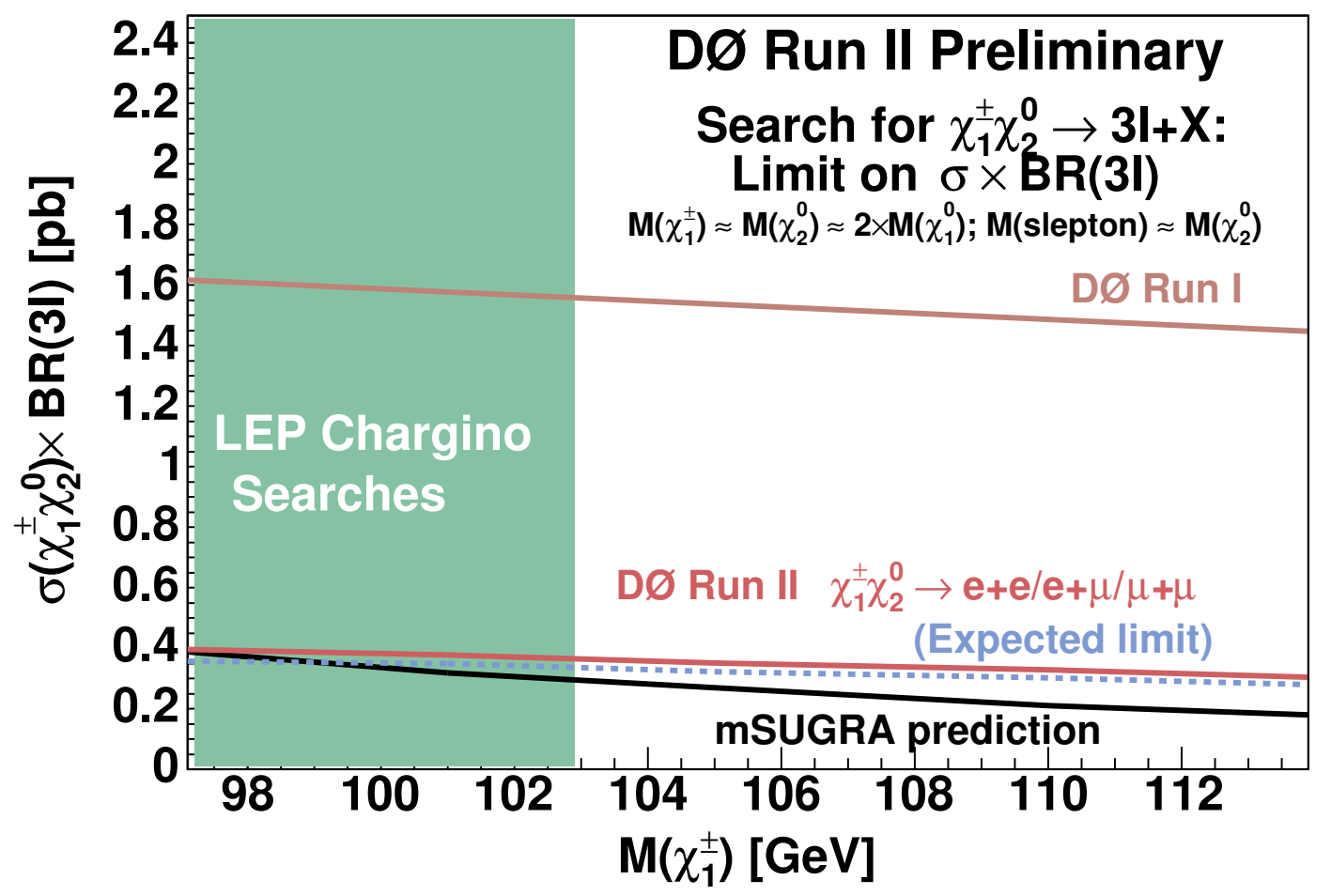

Figure 4.24: Limits on the total cross section for associated chargino $\left(\tilde{\chi}_{1}^{ \pm}\right)$and neutralino $\left(\tilde{\chi}_{2}^{0}\right)$ production with leptonic final states set by D $\varnothing$ in Run I (top line) and in this analysis (second from top) in comparison with the expected limit (second from bottom) and the signal cross section predicted for in mSUGRA (bottom line) as a function of the $\tilde{\chi}_{1}^{ \pm}$mass. Chargino masses below $103 \mathrm{GeV} / \mathrm{c}^{2}$ are excluded by direct searches at LEP. The D $\varnothing$ Run I limit was based on searches in the eee, ee $\mu$, e $\mu \mu$, and $\mu \mu \mu$ trilepton final states with between $75 \mathrm{pb}^{-1}$ and $95 \mathrm{pb}^{-1}$ of integrated luminosity. 


\section{Chapter 5}

\section{Conclusions}

The first search for evidence of supersymmetry in the like-sign dimuon channel at D $\varnothing$ has been presented. The analysis began with basic issues of data quality, and culminated in a new limit on the total cross section for associated chargino and neutralino production with leptonic final states. This new limit was reached in combination with other DØ analyses which studied the dielectron and electron plus muon final states.

While there has been tremendous progress, $\mathrm{D} \emptyset$ is currently taking data that will allow these results to be extended. This data, along with further refinements to the analysis, will allow searches beyond existing limits.

\subsection{The Future of the Analysis at DØ}

Future work on this analysis at $\mathrm{D} \varnothing$ will consist of examining data currently being collected at $\mathrm{D} \varnothing$, and improving the analysis in several ways.

One area which will benefit from further study is the more complete exploration of SUSY phase space. The unknown parameters in the theory lead to a wide range of possible cross sections and differing distributions in several key kinematic variables. Monte Carlo samples for many SUSY points are being generated for exploring more 
of the phase space, including portions with the sign of $\mu$ negative, and with differing mass relations between the sparticles, including regions with the mass of the slepton greater than the masses of the chargino and neutralino. Detailed studies of this space will allow increased sensitivity if analysis cuts are fine-tuned in different parts of the phase space.

Improvements in understanding, modeling, and removing backgrounds should be focused on the $b \bar{b}$ background and the WZ background, as they are currently the largest source of background. It may be possible to use Monte Carlo samples to

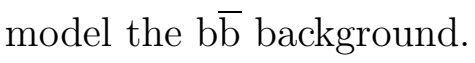

The error from the choice of the PDF set used in the production of signal and background Monte Carlo events needs to be included as well. A procedure is outlined here. The CTEQ6 distribution includes multiple sets of PDFs to be used to estimate the errors. One can generate several Monte Carlo samples with the different sets of PDFs and calculate efficiencies with each. The differences can then be used as an estimate of the error.

The PDF parameters were diagonalized with respect to their linear fit correlations about the best fit point to the full input data sets, and the extremes in that space constitute an uncertainty range for the PDFs. The ranges of uncertainty include the systematic uncertainties as published by many of the input experiments.

Different groups choose to parameterize PDFs differently, and this will affect the parameter values and uncertainties. In another method, one can compare the efficiencies calculated with Monte Carlo events produced with different versions of the CTEQ PDFs or using PDFs produced by other groups, and take these differences as a measure of the systematic error.

Uncertainty at the generator level should be considered as well.

In order to reduce the uncertainty in the background estimation, the Monte Carlo sample sizes need to be increased. Some of the background samples were not large 
enough to leave even one event after all cuts.

To illustrate, consider the uncertainty of b, the background estimate in a channel,

$$
\mathrm{b}=\sigma_{\mathrm{MC}} \times \mathrm{E} \times \mathrm{L}_{\mathrm{data}},
$$

where $\sigma_{\mathrm{MC}}$ is the Monte Carlo cross section of the background process, $\mathrm{E}$ is the efficiency as measured in data, and $\mathrm{L}_{\text {data }}$ is the luminosity of the data sample. E can be estimated as $n / N$, where $n$ is the number of Monte Carlo events observed passing all cuts, and $\mathrm{N}$ is the number of events generated. However,

$$
\mathrm{N}=\sigma_{\mathrm{MC}} \times \mathrm{L}_{\mathrm{MC}}
$$

where $\mathrm{L}_{\mathrm{MC}}$ is the luminosity of the Monte Carlo sample. Therefore, one can write

$$
\mathrm{b}=\mathrm{L}_{\mathrm{data}} \times \sigma_{\mathrm{MC}} \times \frac{\mathrm{n}}{\sigma_{\mathrm{MC}} \times \mathrm{L}_{\mathrm{MC}}}
$$

or

$$
\mathrm{b}=\mathrm{n} \times \frac{\mathrm{L}_{\text {data }}}{\mathrm{L}_{\mathrm{MC}}},
$$

The uncertainty in $\mathrm{b}$ is the uncertainty in $\mathrm{n}$ scaled up by $\mathrm{L}_{\text {data }} / \mathrm{L}_{\mathrm{MC}}$. It is clear that one wants $\mathrm{L}_{\mathrm{MC}} \gg \mathrm{L}_{\text {data }}$. However, when $\sigma_{\mathrm{MC}}$ is large, it is difficult to produce a Monte Carlo sample with enough integrated luminosity.

In this analysis, several of the Monte Carlo samples have integrated luminosity less than or comparable to the data sample (see Table 4.1). The background errors can be reduced by obtaining much larger samples. 


\subsection{The Future Beyond D}

Whether or not supersymmetry is discovered at D $\varnothing$, the Large Hadron Collider (LHC), currently under construction at the CERN laboratory in Geneva, Switzerland, will be a necessary tool in the study of supersymmetry. The LHC will be a proton-proton collider, operating at a center-of-mass energy of $14 \mathrm{TeV}$. If SUSY is not discovered at the Tevatron, it may be discovered at this higher energy. Studies have shown that if supersymmetry is correct, some sign of its existence should be evident at the LHC. If SUSY is discovered at the Tevatron, detailed studies will only be possible at the LHC.

In the next several years, it will be known whether this universe is supersymmetric. 


\section{Appendix A}

\section{L2 Global Worker}

The D $\varnothing$ L2 trigger, described in Chapter 2.3, is an essential part of the data acquisition system [59]. The L2 trigger system is divided into six crates, each containing processors running identical executables (but configured differently). A processor's configuration specifies which worker runs in the processor's executable. The workers correspond to the type of data being processed in the crate.

Five of the L2 crates are preprocessor crates. These crates receive data from the L1 trigger system and the front-end crates. Each crate has a custom-built Beta processor [26] which forms L2 preprocessor objects by running an algorithm tailored to its data. For example, the L2MUC crate receives information about the central muon system and forms muon objects. The preprocessor formats the data properly, and sends the objects to the L2 Global crate.

The L2 Global worker is responsible for making trigger decisions based on the objects identified by the L2 preprocessors. Trigger decisions are made by creating Global physics objects. These objects can be based directly on the objects reported by the preprocessors or can be created by combining objects from different preprocessors. The L2 Global worker imposes cuts on the Global physics objects according to configuration information it receives from the trigger control computer based on 
the trigger list.

\section{A.1 Input}

Data flows from the L2 preprocessor crates into the L2 Global crate (see Figure A.1). The format of the data is described elsewhere [60]. The Beta processor in the L2 Global crate runs an executable which contains the L2 Global worker code.

The L2 Global worker code has access to all of the preprocessor objects as well as information from the L1 framework (L1fwk) for the current event. For each event, the Global worker uses the L1fwk information to decide which algorithms to run on the data from the preprocessors. It then makes a trigger decision, and returns this decision to the L2 framework code which passes it to the trigger framework. Thus, each event must either pass or fail the L2 trigger, and this decision is based on the input data and the information from the trigger list.

The trigger list specifies which trigger conditions L2 will impose for each run. The trigger list can change as frequently as every run, but attempts are made to achieve stability in the trigger list to reduce complexity at the analysis level. All of the possible configuration values are stored in the trigger database, and the trigger list is formed by choosing particular values for all of the variable parameters. The trigger list is downloaded to the L2 Global crate by the trigger control computer, which receives its instructions from COOR, the system responsible for coordinating the distribution of all the online data acquisition information to all the online crates.

\section{A.2 Trigger Bits and Scripts}

The most critical piece of L1fwk information is the L1 trigger mask. This is the record of which L1 bits fired (the reason the current event passed the L1 trigger). The L1 


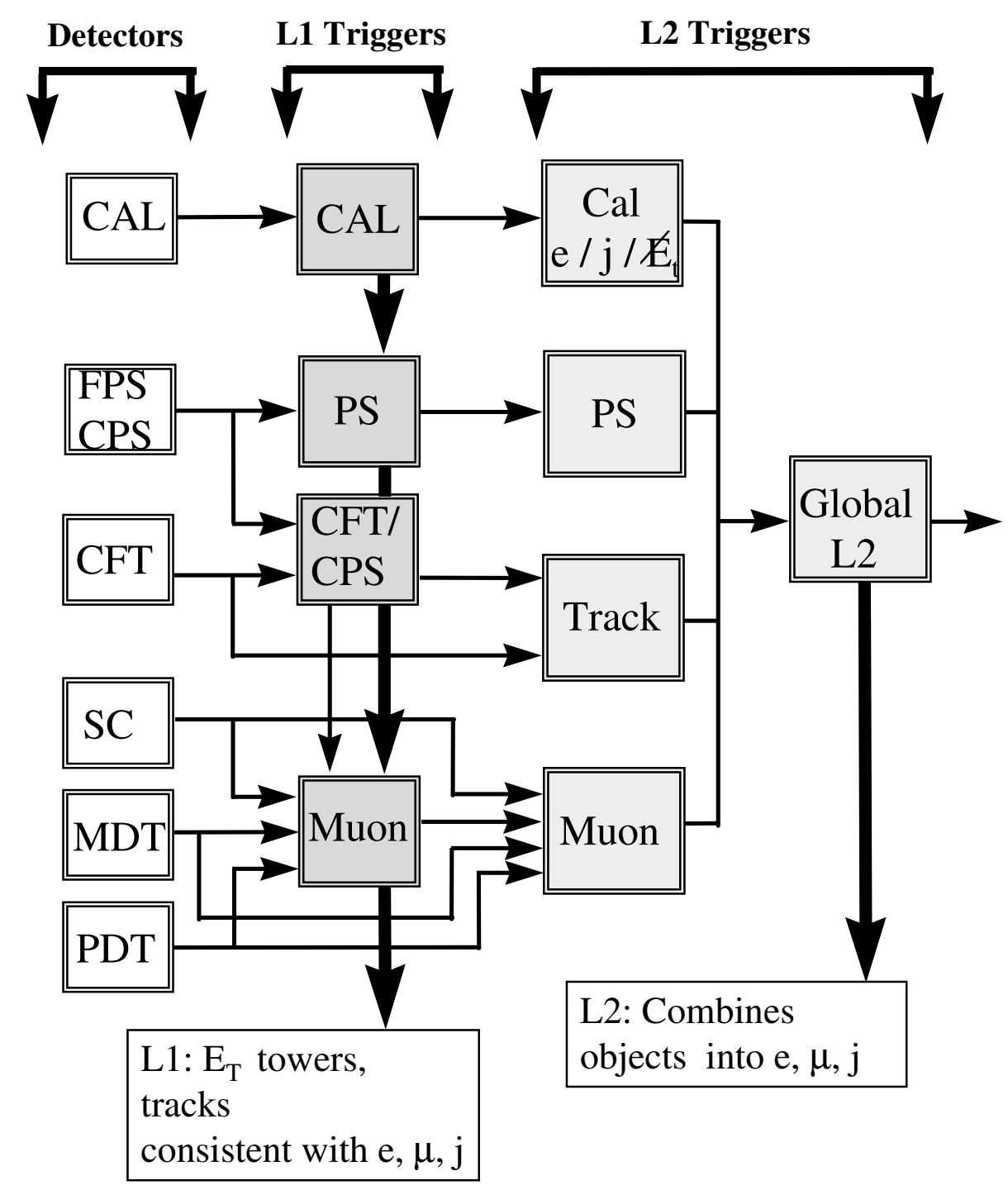

Figure A.1: Data flow from the detectors to the L2 Global worker. 
trigger mask is a collection of 128 bits, one for each L1 trigger condition.

At L1, as many as 128 different conditions can be programmed to fire the L1 trigger. For each event that passes, the bits that passed are recorded and sent to L2. The L2 Global worker begins its work every event by checking which of the L1 trigger bits fired. The trigger list specifies which L2 script is associated with which L1 bit.

The L2 script is the trigger condition that must be satisfied in order for the L2 trigger to fire for a given L1 bit. There is a one-to-one correspondence between L1 bits and L2 scripts. The trigger conditions at each level are closely related, and form a coherent trigger condition. An L2 script is defined, which is related to a L1 bit. Once the L1 bit fires, the corresponding L2 script runs. For example, events with a calorimeter energy requirement at L1 may have an electron requirement at L2.

The L2 script is specified by a number of filters and a minimum number of objects required to pass each filter. The simplest script has one filter and a minimum number of objects required to pass that filter. An example is a script with an electromagnetic object filter and a minimum of two objects. This script passes if there are two electromagnetic objects in the event that satisfied the conditions of the EM filter.

If any script passes, the event passes L2, and is processed by the L3 trigger. Events are only written to tape if they satisfy L1, L2, and L3 trigger conditions.

\section{A.3 Tools and Filters}

Tools and filters are the main parts of the L2 Global worker. The filters make up the scripts described in Section A.2. The filters in turn rely on tools.

Tools are $\mathrm{C}++$ classes which build a specific type of L2 Global object. The electromagnetic object (EM) tool builds L2 Global EM objects. The muon tool builds L2 Global muon objects. This building of objects is based on the preprocessor objects.

A tool starts with a list of preprocessor objects. It can then apply selection criteria 
to decide which preprocessor objects should be made into Global objects. It can also correlate information from two preprocessors by combining two preprocessor objects to form one Global object. An example of this is the combination of a track object and an EM object that come from different preprocessors, but refer to the same real electron that entered the $\mathrm{D} \varnothing$ detector. The tools are written to be flexible, and are configurable through the trigger list. For the above example, one trigger list parameter specifies whether or not EM objects from the preprocessor should be matched to tracks from the tracking system.

The tools produce lists of Global objects. The filters then use these lists of Global objects to make a trigger decision by imposing trigger requirements on the objects. The requirements are based on the trigger list. For the case of an EM object, the electromagnetic fraction, transverse momentum, and isolation are examples of variables that can be required to have values above or below specified thresholds in order to pass the filter.

The filter generates its own list of objects which have satisfied the trigger criteria. The script then checks to see if there are at least the minimum number of objects required to pass the script.

\section{A.3.1 Tools and Filters Currently Available}

Tables A.1, A.2, A.3, A.4 and A.5 summarize the currently available tools and filters and their parameters.

\section{A.3.2 Tools and Filters Used in this Analysis}

In the analysis described in this dissertation, two different L2 trigger requirements are used. The first is the requirement of one medium L2 muon. This is implemented by configuring a L2 muon tool which produced L2 muons based on the list of muons 


\begin{tabular}{|c|c|c|}
\hline Tool & Parameter & Description \\
\hline Muon & $\begin{array}{l}\text { L1PTTHRESH } \\
\text { REQUIRETRACK } \\
\text { TRACKWINDOWIPHI }\end{array}$ & $\begin{array}{l}\text { Minimum } \mathrm{L} 1 \mathrm{p}_{\mathrm{T}} \\
\text { Is match to track required? } \\
\text { Maximum distance to } \\
\text { matching track in } \phi\end{array}$ \\
\hline EM & $\begin{array}{l}\text { MINSINGLETOWERET } \\
\text { REQUIRETRACK } \\
\text { TRACKFILTER } \\
\text { TRACKWINDOWIPHI } \\
\text { REQUIRECPS } \\
\text { CPSWINDOWIETA } \\
\text { CPSWINDOWIPHI } \\
\text { MAXEM }\end{array}$ & $\begin{array}{l}\text { Minimum } \mathrm{E}_{\mathrm{T}} \\
\text { Threshold for which, if cen- } \\
\text { tral cluster } \eta \text { neighbor is be- } \\
\text { low, it will be turned into } 2 \\
\text { separate EM tower objects } \\
\text { Same for central cluster } \phi \\
\text { neighbor } \\
\text { Same for forward cluster } \eta \\
\text { neighbor } \\
\text { same for forward cluster } \phi \\
\text { neighbor } \\
\text { Value for which if EM frac- } \\
\text { tion is greater, it will turn } \\
\text { that cluster into two EM } \\
\text { tower objects } \\
\text { In } \mathrm{E}_{\mathrm{T}} \text { for single tower EM } \\
\text { object. Overrides MINET if } \\
\text { MINNEIGHBORET is true } \\
1=\text { Require a CFT match } \\
\text { found ( } 0 \text { is false) } \\
\text { Track filter } \\
\phi \text { match window track } \\
1=\text { require a CPS match (0 } \\
\text { is false) } \\
\eta \text { match window with CPS } \\
\phi \text { match window with CPS } \\
\text { Maximum number allowed } \\
\text { to pass }\end{array}$ \\
\hline Jet & MINET & Minimum $\mathrm{E}_{\mathrm{T}}$ \\
\hline \multicolumn{3}{|l|}{ MET } \\
\hline MJT & MINET & $\begin{array}{l}\text { Minimum } \mathrm{E}_{\mathrm{T}} \text { of jets to be in- } \\
\text { cluded in calculation }\end{array}$ \\
\hline
\end{tabular}

Table A.1: L2 tools currently available. For each tool, the configurable parameters are listed, along with a brief description [61]. 


\begin{tabular}{|c|c|c|}
\hline Tool & Parameter & Description \\
\hline Track & $\begin{array}{l}\text { MINET } \\
\text { TRACKSOURCE } \\
\text { REQUIREL1ISO } \\
\text { REQUIREL1PS } \\
\text { L2ISOTYPE } \\
\\
\text { MAXCHISQ } \\
\text { IPSIG }\end{array}$ & $\begin{array}{l}\text { Minimum } \mathrm{E}_{\mathrm{T}} \\
\text { Type of input track } \\
\text { Require L1 isolation confir- } \\
\text { mation } \\
\text { Require L1 preshower confir- } \\
\text { mation } \\
\text { Type of L2 isolation re- } \\
\text { quired. } 0=\text { no requirement, } \\
1 \text { or } 2=\text { require } 1 \text { - or } 3 \text { - } \\
\text { prongs, } 3=\text { require } 1 \text {-prong } \\
\text { Maximum } \chi^{2} \text { allowed } \\
\text { Minimum impact parameter } \\
\text { significance }\end{array}$ \\
\hline InvMass & $\begin{array}{l}\text { MININVMASS } \\
\text { NFILTERS } \\
\text { FILTER0 } \\
\text { FILTER1 } \\
\end{array}$ & $\begin{array}{l}\text { Minimum mass } \\
\text { Number of input filters } \\
\text { First filter } \\
\text { Second filter }\end{array}$ \\
\hline Commission & & \\
\hline
\end{tabular}

Table A.2: L2 tools currently available. For each tool, the configurable parameters are listed, along with a brief description [61].

from the two L2 muon preprocessors.

The muon tool makes Global muons based on each of the preprocessor muons it receives. The muon tool also determines the quality of each muon. A L2 muon filter is then configured to loop over all of these muons, and pass any that are of medium quality or better.

The second L2 trigger used in this analysis is similar, except that it uses a L2 muon filter to require at least two medium muons in order to pass. In addition, an $\eta-\phi$ separation filter is used to check the separation in $\eta$ and $\phi$ between the muons passing the muon filter. The separation filter imposes the requirement that for the event to pass, two L2 muons have to be separated by at least 0.15 in $\eta$ or 0.24 in $\phi$. 


\begin{tabular}{|c|c|c|}
\hline Filter & Parameter & Description \\
\hline Muon & $\begin{array}{l}\text { MINET } \\
\text { QUALITY } \\
\text { PROMPT } \\
\text { SIGN } \\
\text { TOOL }\end{array}$ & $\begin{array}{l}\text { Minimum } \mathrm{E}_{\mathrm{T}} \\
\text { Minimum quality (based on } \\
\text { number of hits) } \\
\text { Minimum timing quality } \\
\text { (based on scintillator times) } \\
\text { Required sign of muon to } \\
\text { pass } \\
\text { Input tool }\end{array}$ \\
\hline EM & $\begin{array}{l}\text { EMFRAC } \\
\text { ISOFRAC } \\
\text { MINET } \\
\text { MAXEM } \\
\text { TOOL }\end{array}$ & $\begin{array}{l}\text { Minimum EM fraction } \\
\text { Maximum isolation fraction } \\
\text { Minimum } \mathrm{E}_{\mathrm{T}} \\
\text { Maximum number allowed } \\
\text { to pass } \\
\text { Input tool }\end{array}$ \\
\hline Jet & $\begin{array}{l}\text { MINET } \\
\text { MAXJETS } \\
\text { TOOL }\end{array}$ & $\begin{array}{l}\text { Minimum } \mathrm{E}_{\mathrm{T}} \\
\text { Maximum number of jets al- } \\
\text { lowed } \\
\text { Input tool }\end{array}$ \\
\hline Track & $\begin{array}{l}\text { MINET } \\
\text { QUALITY } \\
\text { IP } \\
\text { IPSIG } \\
\text { TOOL }\end{array}$ & $\begin{array}{l}\text { Minimum }_{\mathrm{T}} \\
\text { Minimum quality } \\
\text { Minimum impact parameter } \\
\text { Minimum impact parameter } \\
\text { significance } \\
\text { Input tool }\end{array}$ \\
\hline MET & $\begin{array}{l}\text { MINMET } \\
\text { TOOL }\end{array}$ & $\begin{array}{l}\text { Minimum } \mathrm{E}_{\mathrm{T}} \\
\text { Input tool }\end{array}$ \\
\hline MJT & $\begin{array}{l}\text { MINMJT } \\
\text { TOOL }\end{array}$ & $\begin{array}{l}\text { Minimum } \mathrm{E}_{\mathrm{T}} \\
\text { Input tool }\end{array}$ \\
\hline Eta & $\begin{array}{l}\text { IETAMIN } \\
\text { IETAMAX } \\
\text { FILTER }\end{array}$ & $\begin{array}{l}\text { Minimum ieta } \\
\text { Maximum ieta } \\
\text { Input filter }\end{array}$ \\
\hline Phi & $\begin{array}{l}\text { IPHIMIN } \\
\text { IPHIMAX } \\
\text { FILTER }\end{array}$ & $\begin{array}{l}\text { Minimum iphi } \\
\text { Maximum iphi } \\
\text { Input filter }\end{array}$ \\
\hline
\end{tabular}

Table A.3: L2 filters currently available. For each filter, the configurable parameters are listed, along with a brief description [61]. 


\begin{tabular}{|c|c|c|}
\hline Filter & Parameter & Description \\
\hline PhiSep & $\begin{array}{l}\text { NFILTERS } \\
\text { IPHIMINSEP } \\
\text { IPHIMAXSEP } \\
\text { FILTER0 } \\
\text { FILTER1 }\end{array}$ & $\begin{array}{l}\text { Number of input filters } \\
\text { Minimum } \phi \text { separation value } \\
\text { Maximum } \phi \text { separation } \\
\text { value } \\
\text { First input filter } \\
\text { Second input filter }\end{array}$ \\
\hline EtaSep & $\begin{array}{l}\text { NFILTERS } \\
\text { IETAMINSEP } \\
\text { IETAMAXSEP } \\
\text { FILTER0 } \\
\text { FILTER1 } \\
\end{array}$ & $\begin{array}{l}\text { Number of input filters } \\
\text { Minimum } \eta \text { separation value } \\
\text { Maximum } \eta \text { separation } \\
\text { value } \\
\text { First input filter } \\
\text { Second input filter } \\
\end{array}$ \\
\hline EtaPhiSep & $\begin{array}{l}\text { NFILTERS } \\
\text { IETAMINSEP } \\
\text { IPHIMINSEP } \\
\text { FILTER0 } \\
\text { FILTER1 } \\
\text { FILTER2 } \\
\text { FILTER3 } \\
\end{array}$ & $\begin{array}{l}\text { Number of input filters } \\
\text { Minimum } \eta \text { separation value } \\
\text { Minimum } \phi \text { separation value } \\
\text { First input filter } \\
\text { Second input filter } \\
\text { Third input filter } \\
\text { Fourth input filter }\end{array}$ \\
\hline PhiSepVeto & $\begin{array}{l}\text { IPHIMINVETO } \\
\text { IPHIMAXVETO } \\
\text { NFILTERS } \\
\text { FILTER0 } \\
\text { FILTER1 } \\
\end{array}$ & $\begin{array}{l}\text { Minimum } \phi \text { separation for } \\
\text { veto } \\
\text { Maximum } \phi \text { separation for } \\
\text { veto } \\
\text { Number of input filters } \\
\text { First input filter } \\
\text { Second input filter }\end{array}$ \\
\hline RSep & $\begin{array}{l}\text { NFILTERS } \\
\text { RMINSEP } \\
\text { RMAXSEP } \\
\text { FILTER0 } \\
\text { FILTER1 }\end{array}$ & $\begin{array}{l}\text { Number of input filters } \\
\text { Minimum } \mathrm{R} \quad \text { separation } \\
\text { value } \\
\text { Maximum } \mathrm{R} \text { separation } \\
\text { value } \\
\text { First input filter } \\
\text { Second input filter }\end{array}$ \\
\hline
\end{tabular}

Table A.4: L2 filters currently available. For each filter, the configurable parameters are listed, along with a brief description [61]. 


\begin{tabular}{|c|l|l|}
\hline Filter & Parameter & Description \\
\hline HT & HTMIN & Minimum HT \\
& NFILTERS & Number of input filters \\
& FILTER0 & First input filter \\
& FILTER1 & Second input filter \\
& FILTER2 & Third input filter \\
\hline InvMass & MININVMASS & Minimum invariant mass \\
& MAXINVMASS & Maximum invariant mass \\
& TOOL & Input tool \\
\hline TransMass & MINTRANSMASS & Minimum transverse mass \\
& MAXTRANSMASS & Maximum transverse mass \\
& TOOL & Input tool \\
\hline TimeDelay & & \\
\hline RandomPass & & \\
\hline
\end{tabular}

Table A.5: L2 filters currently available. For each filter, the configurable parameters are listed, along with a brief description [61].

\section{A.4 Output}

The output of the L2 Global worker is the record of what the worker did for that event. This consists first of a trigger mask that records which scripts passed L2. The trigger mask is used by the L3 trigger to decide which L3 scripts it will run on the event. The output also includes a record of all of the Global objects which caused the event to pass. The format of the Global objects is specified in [60].

\section{A.5 Code Design and Structure}

The code for the L2 Global worker is spread over several packages which are part of both the $\mathrm{D} \varnothing$ offline release and the $\mathrm{D} \varnothing \mathrm{L} 2$ online release.

The offline release contains the trigger simulation code which uses a different framework than the online release. Both frameworks have the same functions; to receive the data and run the worker. The L2 Global code is identical in the online and offline release. The L2 Global code is contained in the packages 12gblem, 12gbljet, 
12gbltrack, 12gblmuon, 12gbltau, 12gblgeneric, 12gblbase, 12gblworker, and 12io.

The 12gblbase, 12gblworker, and 12io packages contain the code used to contain the data, pack/unpack it, and run the tools and filters. The L1fwk bits are interpreted here. These include special bits that instruct the worker to treat the event as part of an unbiased sample by passing it regardless of the results of running the L2 scripts. Errors that have been noted by the L2 framework are also interpreted in these packages. The trigger mask from the L1fwk is decoded and the list of L2 scripts to run is prepared. The code runs all the appropriate scripts, gets the L2 decision, fills the output data, and returns the decision to the L2 framework.

The tool and filter code is contained in the remaining packages. The packages are divided only for organizational reasons. The tools all have access to all of the L2 Global data. They each have a parser interface so that they can receive L2 parser configuration data that originates in the trigger list. They use this configuration information to decide how to produce lists of Global objects.

The tools pass the list of objects to the filters. The filters can then generate lists of objects that are subsets of their input lists. It is also possible for a filter to filter the list produced by another filter. Generic filters are stored in the l2gblgeneric package and do just this. An example is the $\eta$-separation filter, which can take a list of objects from any filter as input, and filter those objects based on their separation in $\eta$. 


\section{Appendix B}

\section{L2 Error Logger}

The D $\varnothing$ L2 trigger, described in Chapter 2.3, is an essential part of the data acquisition system. It must be functioning properly in order for the experiment to trigger on and record data. It is therefore important to monitor it closely to ensure the continued flow of data and to make sure that the system is functioning properly. Good monitoring can greatly speed up the diagnosis of and recovery from problems.

L2 is divided into six crates, each containing processors running identical executables (but configured differently), which are responsible for processing data. The code running on these processors is controlled by an executable written in the $\mathrm{C}++$ programming language. When any error or significant event is noted by the software, it can communicate the event by writing a message via the L2 error logging code. The error logging code encapsulates the collecting, sorting, and routing of the er-

ror information. The $\mathrm{C}++$ source code is collected in the packages 12errorlogger and 12moniogen, which are part of the D $\varnothing \mathrm{L} 2$ online release. 


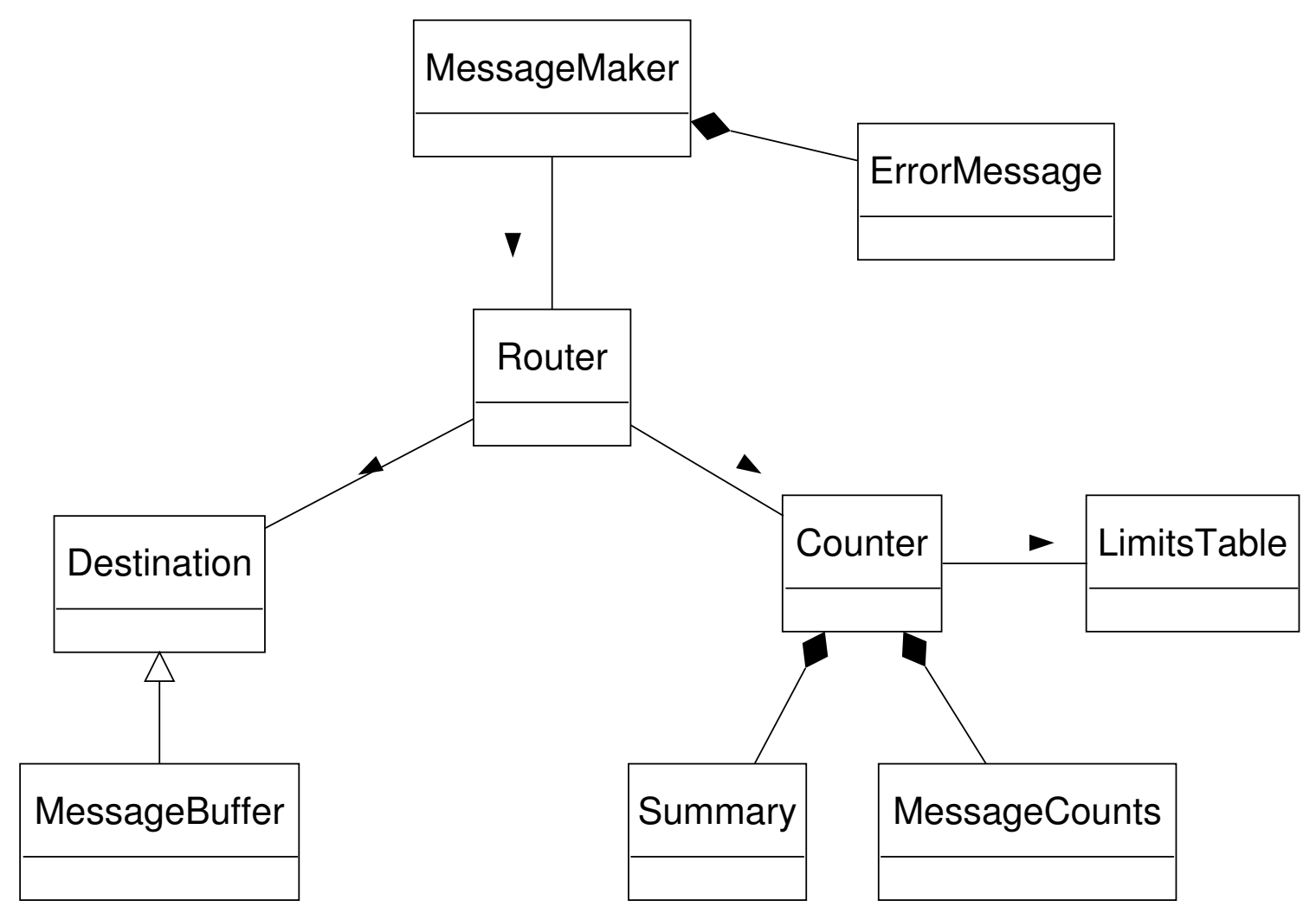

Figure B.1: Simplified Unified Modeling Language (UML) diagram showing the major classes in the 12errorlogger package and the relationships between them.

\section{B.1 Motivation}

There is an offline package called ErrorLogger which is widely used throughout D for collecting, sorting, and routing error information. While this contains all the functionality that is needed for the online L2 system, concerns about speed, memory usage, and code dependencies motivated the decision to create a L2-specific package which implements only the functionality that is needed at L2 according to L2 coding guidelines [62].

The 12errorlogger package has the same interface as the ErrorLogger package. Thus users can write code without distinguishing between the online l2errorlogger and offline ErrorLogger. The switching between the use of the online and offline code is done separately. 


\section{B.2 Architecture}

The MessageMaker class contains the entire user interface. User code directs data to the error logging system by calling functions in the MessageMaker. It is the only class that users of the package will interface their code to directly. One instance of the class, called "errlog" is created by the L2 framework code, and user code must only include one file, "ErrorLog.hpp," to make use of the l2errorlogger. The names "errlog" and "ErrorLog.hpp" were chosen to agree with the names used by the offline ErrorLogger.

The 12errorlogger package contains a header file directory that has files that define the classes used in the l2errorloger. The implementation of the functionality of the classes is contained in files in the src directory. The container classes for the 12errorlogger data are in the 12moniogen package. The main classes that are used in the 12errorlogger package and the relationships between them are shown in a Unified Modeling Language (UML) diagram in Figure B.1.

The MessageMaker receives data from the user code and constructs an error message using the ErrorMessage class. This class contains the information specified by the user: the message name; the message's severity level (Table B.1); a text message which describes the error; and the current bunch and rotation number.

The error message produced by the MessageMaker is passed to the Router class, which is responsible for its fate. The Router first sends the message to the Counter class, which keeps track of how many times each message has been logged. The Counter stores each message, how many times it has been logged, and constructs and fills Summary objects. The Counter consults the LimitsTable class which contains configuration information downloaded from the trigger control computer. The LimitsTable can be configured to limit how often messages are logged, or suppress messages based on the number of occurrences or their severity level. The LimitsTable 


\begin{tabular}{|c|c|}
\hline Error Name & Error Severity \\
\hline 0 & ELunspecified \\
1 & ELzeroSeverity \\
2 & ELincidental \\
3 & ELsuccess \\
4 & ELinfo \\
5 & ELwarning \\
6 & ELwarning2 \\
7 & ELerror \\
8 & ELerror2 \\
9 & ELnextEvent \\
10 & ELsevere \\
11 & ELsevere2 \\
12 & ELabort \\
13 & ELfatal \\
14 & ELhighestSeverity \\
\hline
\end{tabular}

Table B.1: The available severity levels in the 12errorlogger.

returns whether the message should be sent on or disregarded. The Counter receives this decision and returns it to the Router.

The Router, after receiving the decision to send the message on, sends the message to the Destination class. The Destination is configurable and can be standard out, a log file, or a MessageBuffer class that writes to a block of memory that is written to the dual port memory every few seconds. The trigger control computer pulls the contents of the buffer periodically. From there, the data can be transferred to the monitoring system. It also gets written to log files and stored. Clients can request the monitoring information from the monitoring system, and use it for control-room displays.

\section{B.3 User Interface}

The user interface for the 12errorlogger starts with the requirement that the file ErrorLog.hpp be included. Then, anywhere in the code, the user can log a message by 
typing

errlog $(<m e s s a g e ~ n a m e>,<m e s s a g e ~ s e v e r i t y>)<<<$ message text $><<$ endmsg; where

- $<$ message name $>$ is any text string the user uses to name the message. This is used as a label, and for the summary information.

- $<$ message severity $>$ is a severity level from Table B.1.

- $<$ message text $>$ is any detailed text string containing the error message. Data can also be logged by typing " $<<$ data".

- endmsg is necessary to terminate the message.

An example of usage is

double chargino_mass $=150$;

errlog("Chargino Found!', ELinfo) 《 'A chargino with mass ',

< chargino_mass $<<$ " has been found" $<$ endmsg; 


\section{Appendix C}

\section{Paddle Board for Hardware Scalers}

The state of the running executable on the main processor in each L2 crate is monitored by hardware scalers that are controlled from within the running executable. There are six L2 crates, each containing Beta processors [26] that run identical executables (but are configured differently), that are responsible for processing data. The code running on these processors is controlled by an executable written in the $\mathrm{C}++$ programming language. As the code moves from one state to another, it changes the output signals to the monitoring system on the Beta. These signals are transmitted off the board on a 68-pin cable (3M part number 10168-8000EE).

The signals from this board are transmitted to a crate which receives monitoring information from many crates. The crate keeps track of the information by incrementing scalers (counters) based on the signals. Field-programmable gate arrays (FPGAs) in the monitoring crate check the values of the signals for each bunch crossing of the accelerator. If the value of a signal is high, the corresponding scaler is incremented. The interface to the monitoring crate is through 24-pin connectors (TYCO electronics part number 501-2407ES). Since the signals coming from the L2 processor are output on a 68-pin cable, conversion to 24-pin cables must be performed to allow interfacing to the monitoring crate. This conversion is done through a passive circuit board 


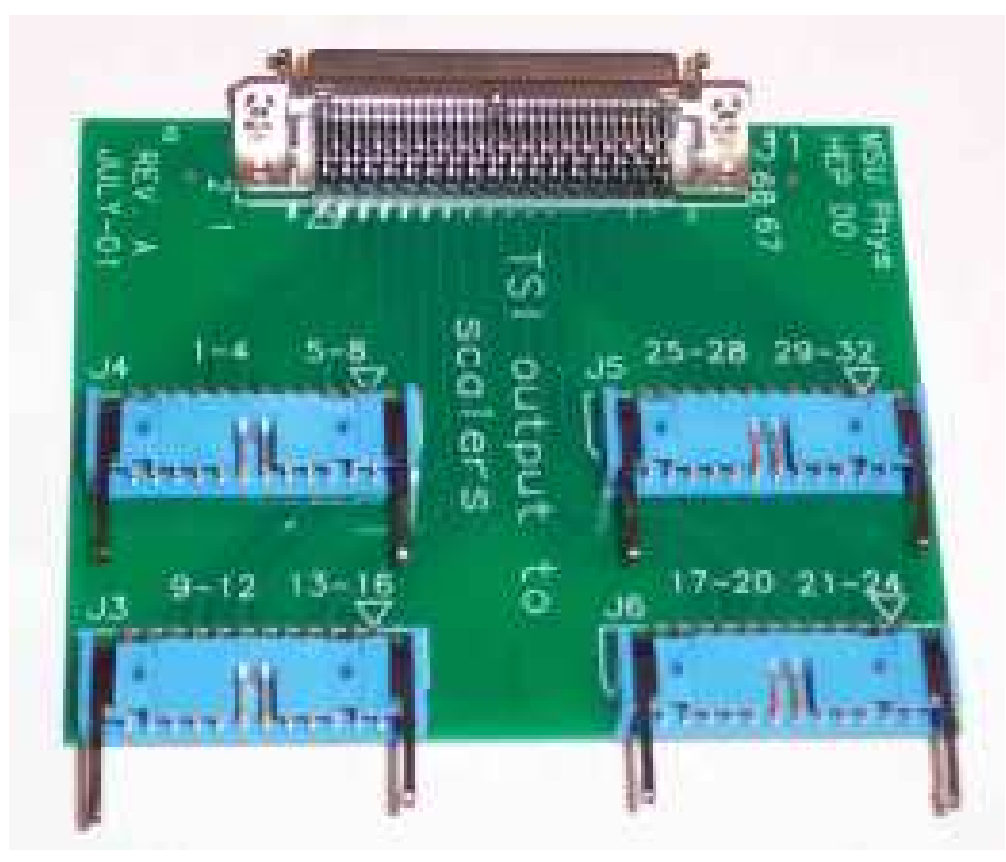

Figure C.1: The paddle board. Picture courtesy of Kathleen Yurkewicz.

(referred to here as the paddle board).

The paddle board is a 4-layer printed circuit board (see Figures C.1 and C.2). It has five connectors. J1 is the 68-pin connector which receives the data from the L2 processor. J3, J4, J5, and $\mathrm{J} 6$ are the 24-pin connectors which transmit the data to the monitoring crate.

The 68-pin cable has four ground wires. The remaining 64 wires transmit 32 ECL signals. Each pair of wires carries the direct and complement signals. The voltage difference between the pair signify an on or an off bit to the receiving scalers. The 32 pairs are routed to the four connectors J3, J4, J5, and J6 on the circuit board. Eight pairs go to each connector, which leaves 8 pins unused on each of the four 16-pin connectors.

The mapping of signals for all the crates to the scalers is kept in a net list. The net list consists of rows of information, separated into columns. Each row corresponds to a net, or one wire on a cable. Column 1 always says "NET". Column 2 is a name ending in DIR or COMP for direct or complement. Column 3 is the origin of the net, 


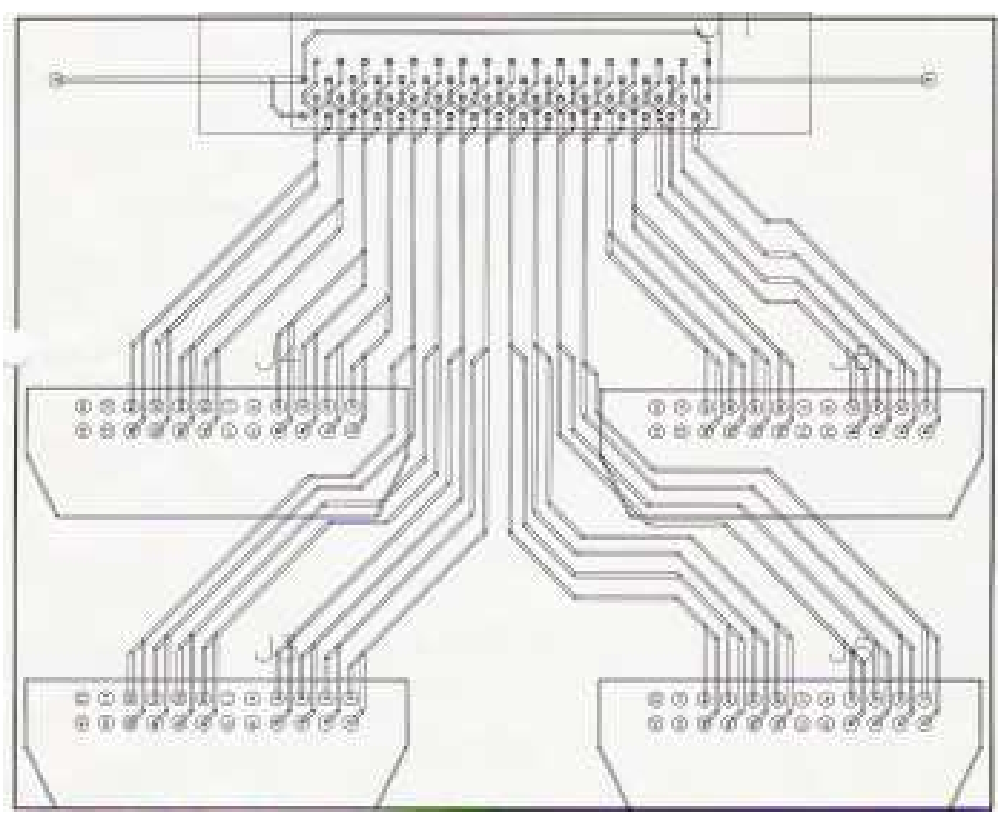

Figure C.2: Design diagram of the paddle board, showing the connectors and traces connecting them.

a connector, and a pin number. Column 4 is the destination of the net. Column 5 is the FPGA that receives this signal. Column 6 is the control signal on that FPGA.

The monitoring data will contain the counts data from all 384 of the L2 scalers, with 4 scalers per FPGA, 16 FPGAs per scaler card, and 6 scaler cards. The scalers on each FPGA are numbered 0 to 3 . The FPGAs that contain scalers on each scaler card are numbered 1 to 16.

Part of the net list looks like:

\#

$\begin{array}{lllllll}\text { NET } & \text { 'ALPHA_IN_01_DIR' } & \text { J1-03 } & \text { J4-19 } & \text { \# FPGA \#6 } & \text { CS } 3 \\ \text { NET } & \text { 'ALPHA_IN_01_COMP' } & \text { J1-04 } & \text { J4-20 } & \text { \# FPGA \#8 } & \text { CS } 3 \\ \# & & & & & & \\ \text { NET } & \text { 'ALPHA_IN_02_DIR' } & \text { J1-05 } & \text { J4-17 } & \text { \# FPGA \#6 } & \text { CS } 2 \\ \text { NET } & \text { 'ALPHA_IN_02_COMP' } & \text { J1-06 } & \text { J4-18 } & \text { \# FPGA \#8 } & \text { CS } 2\end{array}$

\# 


$\begin{array}{llllllll}\text { NET } & \text { 'ALPHA_IN_03_DIR' } & \text { J1-07 } & \text { J4-15 } & \text { \# FPGA \#6 } & \text { CS } 1 \\ \text { NET } & \text { 'ALPHA_IN_03_COMP' } & \text { J1-08 } & \text { J4-16 } & \text { \# FPGA \#8 } & \text { CS } 1 \\ \# & & & & & & \\ \text { NET } & \text { 'ALPHA_IN_04_DIR' } & \text { J1-09 } & \text { J4-13 } & \text { \# FPGA } & \text { F6 } & \text { CS } & 0 \\ \text { NET } & \text { 'ALPHA_IN_04_COMP' } & \text { J1-10 } & \text { J4-14 } & \text { \# FPGA } & \text { F8 } & \text { CS } & 0\end{array}$

The up-to-date full net list and meaning of each bit are documented on the L2 monitoring web page [61].

The monitoring data is automatically collected every five seconds and displayed in the control room as part of the L2 monitoring display. Pie charts are generated which show the amount of time spent in each state. A pie chart and the corresponding legend for the Global worker are shown in Figure C.3. Distributions can also be made that show how long the processor spends in each state (on an event-by-event basis). 


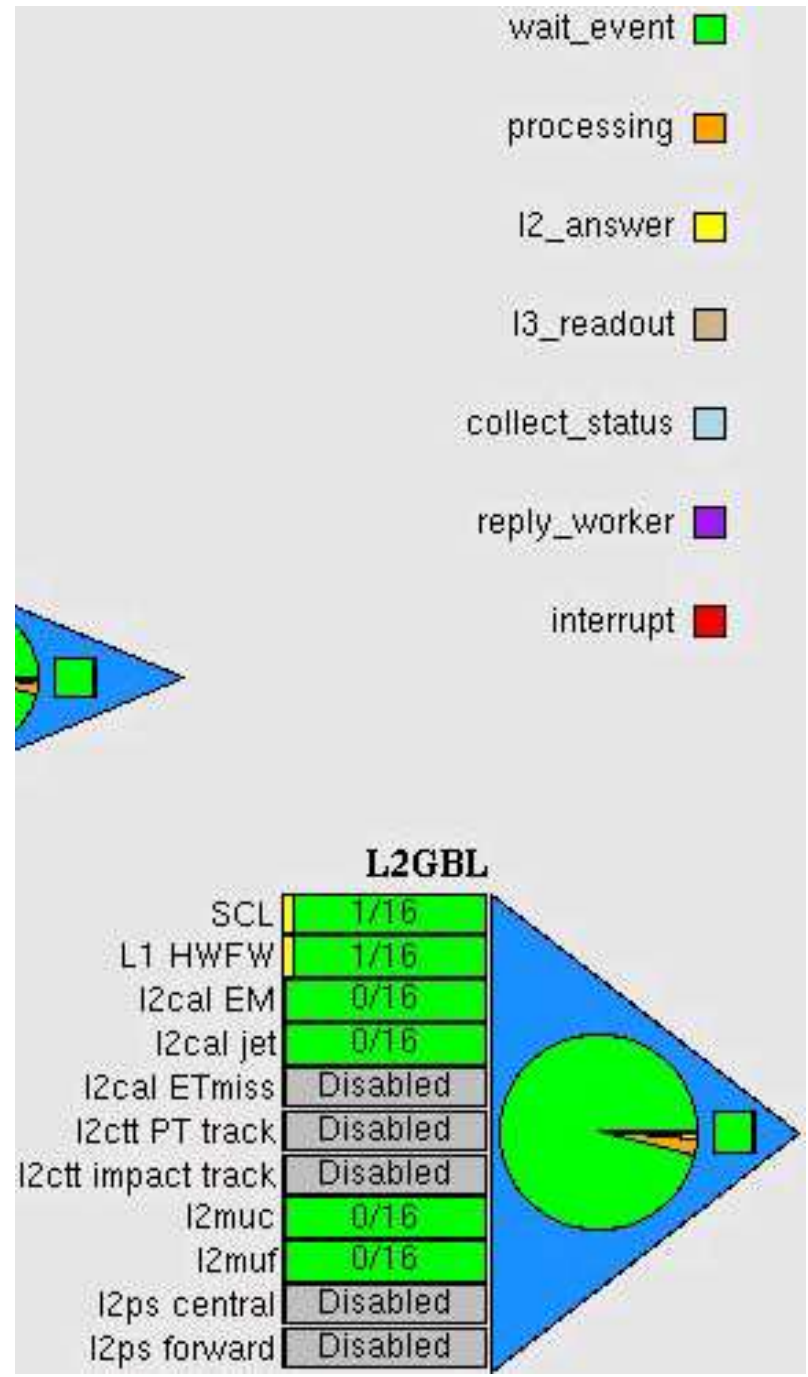

Figure C.3: A pie chart and corresponding legend showing the amount of time spent in each state for the Global worker. 


\section{Bibliography}

[1] F. Halzen and A. Martin. Quarks and Leptons: An Introductory Course in Modern Particle Physics. John Wiley and Sons, New York, 1984.

[2] C. Quigg. Gauge Theories of the Strong, Weak, and Electromagnetic Interactions. The Benjamin/Cummings Publishing Company, London, 1983.

[3] G. Kane. Modern Elementary Particle Physics. Addison-Wesley Publishing Company, Reading, MA, 1993.

[4] D. Diakonov, V. Petrov, and M. Polyakov. Z. Phys. A, 359:305, 1997.

[5] P. Higgs. Phys. Lett., 12:132, 1964.

[6] S. Dawson. hep-ph, 9612229, 1997.

[7] M. Drees. hep-ph, 9611409, 1996.

[8] A. Lyon. The Basics of Supersymmetry. DØ Note 2523, 1995.

[9] S. Martin. hep-ph, 9709356, 1999.

[10] V. Trimble. Ann. Rev. Astron. Astrophys., 25:425, 1987.

[11] J. Thompson. Introduction to Colliding Beams at Fermilab. FERMILAB-TM1909, 1994.

[12] Accelerator Concepts. http://www-numi.fnal.gov/workgrps/protonwg/accel_reference.html, 2002.

[13] Main Injector Rookie Book.

http://www-bdnew.fnal.gov/operations/rookie_books/rbooks.html, 2003.

[14] T. Ferbel. A Brief Description of the DØ Detector in Run II. http://www-d0.fnal.gov/Run2Physics/WWW/templates/detector.tex, 2004.

[15] The D $\varnothing$ Collaboration. The DØ Upgrade: The Detector and Its Physics. 1996.

[16] S. Snyder. Measurement of the Top Quark Mass at DØ. PhD thesis, State University of New York at Stony Brook, 1995. 
[17] S. Abachi et al. Nucl. Instrum. Methods Phys. Res. A, 338:185, 1994.

[18] V. Abazov et al. in preparation for submission to Nucl. Instrum. Methods Phys. Res. A.

[19] M. Begel, D. Edmunds, P. Laurens, and R. Partridge. D $\varnothing$ Luminosity in Run 2: Delivered. DØ Note 3970, 2003.

[20] D $\varnothing$ Upgrade Collaboration. D $\varnothing$ Silicon Tracker Technical Design Report. http://d0server1.fnal.gov/projects/silicon/www/tdr_final.ps, 1994.

[21] D $\varnothing$ Collaboration. The D $\varnothing$ Upgrade: Central Fiber Tracker Technical Design Report. http://d0server1.fnal.gov/projects/SciFi/cft_home.html, 1999.

[22] M. Adams et al. Design Report of the Central Preshower Detector for the DØ Upgrade. http://d0server1.fnal.gov/users/qianj/CPS/doc/dn3104.pdf, 1996.

[23] A. Gordeev et al. Technical Design Report of the Forward Preshower Detector for the DØ Upgrade. DØ Note 3445, 1998.

[24] B. Baldin et al. Technical Design Report of the Central Muon System. DØ Note 3365, 1997.

[25] G. Alexeev et al. Technical Design Report for the DØ Forward Muon Tracking Detector Based on Mini-drift Tubes. DØ Note 3366, 1997.

[26] B. Hirosky. DØ L2 Beta Project. http://galileo.phys.virginia.edu/ rjh2j/12beta/, 2004.

[27] M. Adams, R. Hirosky, R. Martin, N. Varelas, G. Di Loreta, and D. Owen. Level-2 Calorimeter Technical Design Report. DØ Note 3651, 1999.

[28] M. Fortner. Technical Design Report for the Muon Level-2 Preprocessor. http://niuhep.physics.niu.edu/ ${ }^{\sim}$ fortner/d0/trig/muon2/tdr.ps, 1997.

[29] R. Angstadt et al. The DZERO Level 3 Data Acquistion System. 2003.

[30] DØ Collaboration. d0reco. http://www-d0.fnal.gov/Run2Physics/WWW/algorithm.htm, 2004.

[31] G. Borissov. Ordering a Chaos or...Technical Details of AA Tracking. http://www-d0.fnal.gov/global_tracking/talks/20030228/talk-adm-030228.ps, 2004 .

[32] A. Khanov. HTF: Hstogramming Method for Finding Tracks. DØ Note 3778, 2000 . 
[33] A. Schwartzman and M. Narain. Probabilistic Primary Vertex Selection. DØ Note 4042, 2002.

[34] L. Feligioni et al. Update on b-quark jet identification with Secondary Vertex reconstruction using D $\varnothing$ reco version p14. DØ Note 4414, 2004.

[35] D. Bloch et al. Performance of the JLIP b-tagger in p14. DØ Note 4348, 2004.

[36] R. Demina, A. Khanov, and F. Rizatdinova. B-tagging with Counting Signed Impact Parameter Method. DØ Note 4049, 2002.

[37] Heavy Flavour (b and c quark) Identification Group. Soft Lepton (muon) tagging method.

http://www-d0.fnal.gov/phys_id/bid/d0_private/certification/p14/ muonjet/SLT_v1.html, 2004.

[38] C. Clement et al. MuonID Certification for p14. DØ Note 4350, 2004.

[39] D. Whiteson and M. Kado. Muon Isolation Studies. DØ Note 4070, 2003.

[40] O. Peters. Measurement of the b-jet cross section at $\sqrt{s}=1.96 \mathrm{TeV}$. PhD thesis, Universiteit van Amsterdam, 2003.

[41] A. Melnitchouk. Search for non-SM Light Higgs Boson in the $h \rightarrow \gamma \gamma$ Channel. $\mathrm{PhD}$ thesis, Brown University, 2004.

[42] L. Sawyer and A. Stone. Missing ET Reconstruction: Variables and Methods. DØ Note 3957, 2003.

[43] J. Nachtman, D. Saltzberg, and M. Worcester for the CDF collaboration. hep-ex, 9902010, 1999.

[44] D $\varnothing$ New Phenomena Group. NP group skimming web page. http://www-clued0.fnal.gov/ ${ }^{\sim}$ gusbroo/d0_private/skims-to-trees.html, 2004.

[45] D $\varnothing$ Common Samples Group. CS group home page. http://www-d0.fnal.gov/Run2Physics/cs/index.html, 2004.

[46] J. Elmsheuser. Search for the Higgs boson in $\mathrm{H} \rightarrow \mu^{+} \nu_{\mu} \mu^{-} \bar{\nu}_{\mu}$ decays at $\mathrm{D} \varnothing$ in Run II. DØ Note 4386, 2004.

[47] T. Sjostrand, L. Lonnblad, S. Mrenna, and P. Skands. hep-ph, 0308153, 2003.

[48] M.L. Mangano, M. Moretti, F. Piccinini, R. Pittau, and A. Polosa. hep-ph, 0206293, 2002.

[49] B. Abbott et al. Phys. Rev. Lett., 85, 2000.

[50] W. Beenakker, M. Klasen, M. Krämer, T. Plehn, M. Spira, and P.M. Zerwas. hep-ph, 9906298, 1999. 
[51] U. Chattopadhyay and P. Nath. Phys. Rev. Lett., 86, 2001.

[52] U. Blumenschein and V. Buescher. Search for the Associated Production of Chargino and Neutralino in Final States with Two Electrons and an Additional Lepton. DØ Note 4405, 2004.

[53] M. Hohlfeld. Search for Associated Chargino/Neutralino Production in the e- $\mu$ + X Final State. D $\varnothing$ Note 4406, 2004.

[54] M. Binder and R. Strohmer. Search for the Associated Chargino-Neutralino Production in Final States with Two Muons and an Additional Lepton. DØ Note 4567, 2004.

[55] U. Blumenschein, V. Buescher, R. Hauser, M. Hohlfeld, J. Linnemann, R. Moore, and A. Yurkewicz. Search for the Associated Production of Chargino and Neutralino in Final States with Three Leptons. DØ Note 4368, 2004.

[56] T. Junk. Nucl. Instrum. Methods Phys. Res. A, 434:435, 1999.

[57] B. Abbott et al. Phys. Rev. Lett., 80:8, 1998.

[58] F. Abe et al. Phys. Rev. Lett., 80:5275, 1998.

[59] D. Edmunds, S. Gross, P. Laurens, J. Linnemann, and R. Moore. Technical Design Report for the Level 2 Global Processor. DØ Note 3402, 1998.

[60] J. Linnemann, R. Moore, and A. Yurkewicz. Format and Contents of L2 Global Input and Output Objects. http://www-clued0.fnal.gov/L2/global/, 2004.

[61] L2 Trigger Group. L2 Web Page. http://www.pa.msu.edu/hep/d0/12/, 2004.

[62] R. Moore. D $\varnothing$ Run II L2 Programming Rules. http://www-clued0.fnal.gov/L2/L2CodingGuidelines.pdf, 1999. 